INSTITUTO DE Física E QUímICA DE SÃo CARLos

DEPARTAMENTO DE FíSICA E CIÊNCIA DOS MATERIAIS

UNIVERSIDADE DE SÃO PAULO

\author{
"PROBLEMAS BIDIMENSIONAIS DE CARGA \\ ESPACIAL EM GEOMETRIAS PLANO-PLANO \\ E ASSEMELHADAS"
}

Ismael Francisco Dantas

Tese apresentada ao Instituto de Física e Quimica de São Carlos, para a obtenção do Título de Doutor em Ciências: Física Aplicada

Orientador

Prof. Dr. Guilherme Fontes Leal Ferreira

SÃO CARLOS

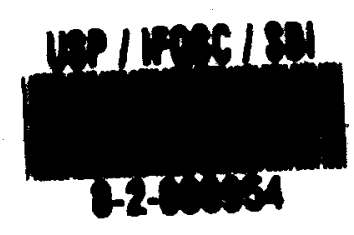

1992

SERVIÇO DE BIBLIOTECA E INFORMACAO - IFOSC

FISICA 


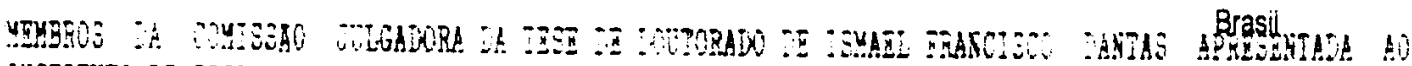

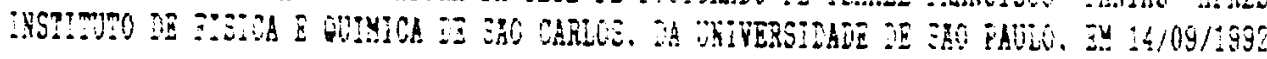

MOISSAO WUUALORA:

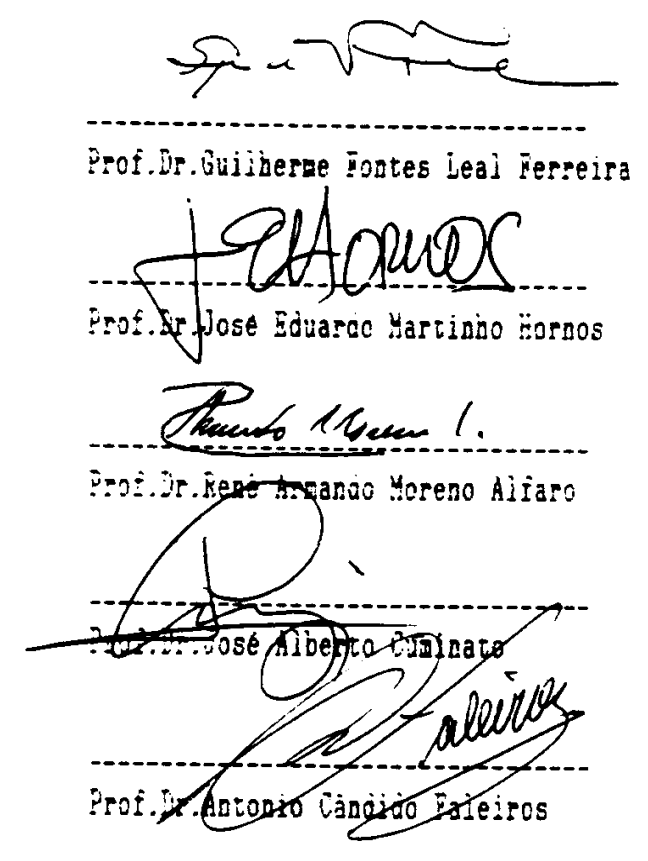


A minha esposa

Teresa Virgínia

Aos meus filhos

Clarissa

Marcílio

Igor

Márcia

Marcos Dimitri. 


\section{Agradecimentos}

Ao Prof. Dr. Guilherme F. Leal Ferreira, pela orientação segura e constante estímulo à superação das dificuldades encontradas.

Ao Prof. Dr. José Alberto Cuminato (Poti), pelas discussōes e sugestōes em aspectos importantes deste trabalho.

Aos Professores Dr. Roberto M. Faria e Dr. José Alberto Giacometti, pelo incentivo e amizade.

Aos demais Professores e Funcionários do Grupo de Eletretos que, direta ou indiretamente, contribuiram para a realização deste trabalho.

Ao casal amigo Auro e Sônia Tanaka, pelo apoio constante, hospitalidade e generosa amizade.

Aos colegas João Mariz, Paraná e Marta, José Ribeiro, Helder, Edinilton, Haroldo e Malmonge, pelo convivio e amizade.

Aos colegas Professores do Departamento de Física da Universidade Federal do Piaui que suportaram minhas atividades docentes durante a realização deste trabalho.

À FUFPI e ao IFQSC-USP, pela oportunidade. 


\title{
Agradecimento Especial
}

\author{
A Profa. Dra. Mariângela T. Figueiredo, pela valiosa colaboração, pelo \\ interesse sempre renovado e paciência com os quais me assistiu em \\ todas as fases deste trabalho. Digo-lhe, pois, e ao Prof. Guilherme, que \\ se melhor não fiz foi por causa das minhas próprias limitações.
}




\section{Resumo}

A finalidade principal deste trabalho é o da obtenção de soluçōes numéricas de movimento de carga espacial livre em que as grandezas pertimentes dependem de mais de uma coordenada espacial (casos não unidimensionais). Mais especificamente, os problemas estudados versam sobre casos em que um eletródio plano a um dado potencial emite carga não uniformemente ao longo de sua extensão e procura-se obter a deformação das linhas de campo dá resultantes, no espaço até um outro eletródio aterrado disposto paralelamente ao primeiro. Um método baseado em soluções tentativas bem como um outro global usando a "transformação hodográfica" recentemente formulada por Budd e Wheeler são apresentados. É também realizado um estudo breve sobre a validade da muito empregada "suposição de Deutsch". 


\begin{abstract}
The aim of the present work is to obtain numerical solutions of free space charge motion in cases in which the pertinent quantites depend on more than one space coordinate (non uni-dimensional cases). More specifically, the problems under concern here are those in which a plane electrode, at a constant potential, emit charge in a non-uniform way along them and the resultant deformation of the field lines, in the space between it and another plane grounded electrode parallelly disposed, is looked for. A method based on guessed solutions and also a global one using the hodographic transformation recently developed by Budd and Wheeler are presented. A brief study of the validity of the of ten employed "Deutsch assumption" is also carried out.
\end{abstract}




\section{índice}

CAPÍTULO I - INTRODUÇÃO. 1

CAPÍTULO II - EQUAÇõES DO PROBLEMA DE CARGA ESPACIAL 3

2.1 - Formas Dimensionais ............................................................3

2.2 - Formas Adimensionais .......................................................4 4

2.3 - Características do Sistema de Equaçōes ....................................5

2.4 - Condições de Contorno ...........................................................6

2.5 - Soluções Exatas .....................................................................8

2.5.1 - Emprego de Soluçōes Exatas ....................................... 10

2.6 - Soluções Aproximadas .................................................................11

CAPÍTULO III - SOLUÇĀo DAS EQUAÇÕES DE CARGA ESPACIAL - UMA APROXIMAÇÃO EM SÉRIES DE TAYLOR TRUNCADAS ............. 17

3.1 - Introdução ..................................................................... 17

3.2 - Determinação de Coeficientes ................................................. 18

3.3 - Sistema de Dois Eletródios Planos ...................................... 20

3.4 - Cálculo de Equipotenciais .................................................. 22

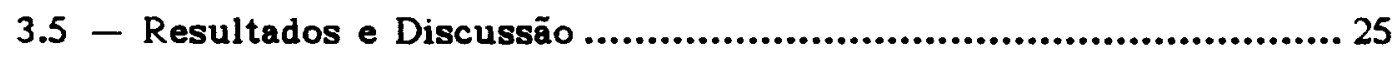

CAPÍTULO IV - O MÉTODO DE BUDD \& WHEELER E AS EQUAÇõES DE CARGA ESPACIAL

4.1 - Introdução.................................................................... 37

4.2 - Condições de Ortogonalidade do Sistema $(\Phi, \Psi)$......................... 38

4.3 - Transformação das Equações sob a Mudança de

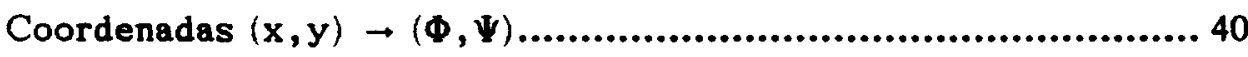

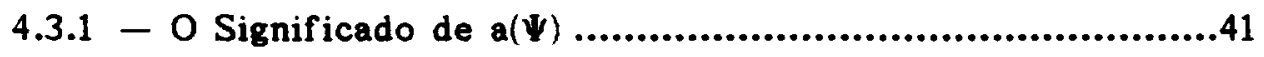

4.3.2 - Condições de Contorno Apropriadas ao Sistema de

Equaçōes (4.11) em um Domínio Simplesmente Conexo ... 42 
CAPÍTULO V - INJEÇĀO DESCONTÍNUA DE CARGA ESPACIAL

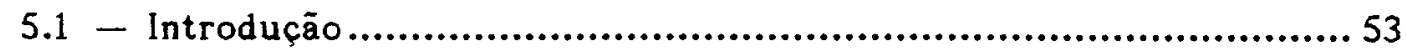

5.2 - As Condições de Contorno em ar ...................................... 56

5.3 - Abordagem de $\Gamma_{1}$ e $\Gamma_{2}$ como Domínios Isolados ........................ 58

5.3 .1 - Região de Laplace .................................................. 58

5.3.2 - Região de Poisson ................................................. 59

5.4 - Os Algoritmos para a Determinação de $\partial \Gamma$............................ 62

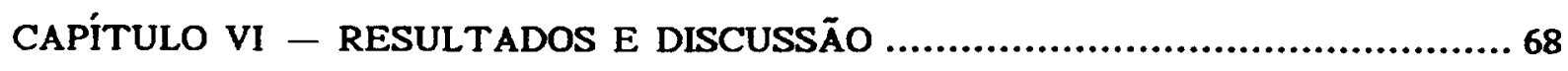

6.1 - Introdução................................................................ 68

6.2 - Sobre a Convergência dos Algoritmos ............................... 70

6.3 - Resultados Numéricos do Caso I e Discussão.............................71

6.4 - Resultados Numéricos para a Linha de Corrente

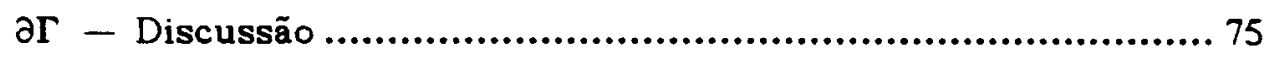

6.5 - Resultados Numéricos do Caso II - Discussão ...................... 87

6.6 - Considerações Finais .................................................. 89

CAPÍTILO VII - A SUPOSIÇÃO DE DEUTSCH EM GEOMETRIAS

SIMPLESMENTE CONEXAS $. . . \ldots \ldots \ldots \ldots \ldots \ldots \ldots \ldots \ldots \ldots \ldots \ldots \ldots \ldots \ldots \ldots . . . \ldots 1$

SUGESTÕES PARA TRABALHO FUTURO ........................... 95

APÊNDICE - A - SOBRE O EMPREGO DAS SUB-ROTINAS NAG

F04AXT E F01BRF ........................................................ 97

APÊNDICE - B - PROGRAMAS FORTRAN .....................................103

REFERÊNCIAS BIBLIOGRÁFICAS ................................121 


\section{CAPITULO I}

\section{Introduçāo}

Os problemas de "carga espacial" estão geralmente ligados ao de transporte de cargas elétricas em isolantes. As cargas emitidas de um eletródio injetor acumulam-se no espaço entre este e o eletródio coletor mantendo-se assim mesmo no regime estacionário. Em simetria planar foram já apresentadas soluçōes para um grande número de problemas quer em regime estacionário ou não. Na maioria das vezes se admite que a velocidade do portador é proporcional à força elétrica sobre ele, o transporte sendo livre ou em presença de armadilhas que podem imobiliza-los temporariamente. Distinguem-se também dois casos: o de muita e o de pouca carga espacial comparadas com as cargas que residem no eletródio emissor. Um amplo estudo - embora com mais ênfase nos estados estacionários é apresentado no livro "Current Injection in Solids" por Lampert \& Mark [1]. Para situações não estacionárias vejam-se os artigos de Many \& Rakavy [2] e Oliveira \& Leal Ferreira [3].

Entre as situaçōes não triviais, isto é, aquelas em que as grandezas pertinentes como o campo elétrico e a densidade de carga dependem de mais de uma coordenada espacial, a única a receber atenção foi a da descarga corona, de montagem frequentemente empregada na prática. Nesta, a descarga disruptiva junto a um condutor filiforme ou pontiagudo, devida à existência de um grande campo elétrico, confina-se às vizinhanças desse condutor, a corrente elétrica sendo na maior parte do espaço de natureza iônica. Pois bem, este problema da descarga corona recebeu algumas abordagens destacando-se as aproximações embasadas na suposição de Deutsch. No cap.II apresentamos as equaçōes que descrevem o regime estacionário do movimento de carga espacial, alguns teoremas, as soluções exatas conhecidas e algumas poucas aproximações analiticas. Vê-se que, em geral, não é mais possivel obter-se soluções analíticas nos casos não triviais, sendo então necessário o emprego de soluçōes numéricas. 
O problema não trivial que estudamos nesta tese é o da emissão não uniforme de carga por um eletródio plano infinito, a um dado potencial. No cap. III estipulamos a densidade de carga e o campo elétrico sobre um eletródio plano emissor e através de um desenvolvimento em série até segunda ordem avançamos em direção ao outro eletródio que não resultará exatamente plano mas o será em uma muito boa aproximação. Os resultados obtidos por este método serão testados por um outro, muito mais sofisticado, desenvolvido por Budd \& Wheeler [4] que consiste em resolver numericamente, depois de uma conveniente mudança de variáveis, o problema de contorno com fronteiras planas vindo da equação de Poisson e o problema hiperbólico de transporte de carga ao longo das linhas de fluxo. Este método está apresentado no cap.IV.

Já no cap. $V$ voltamos ao problema da emissão não uniforme começando a estudar o seguinte caso: dois eletródios planos infinitos estão dispostos paralelamente. Um deles emite cargas com densidade de carga constante até um certo ponto (ou linha) em sua superficie. Qual será o locus da última linha de corrente e de suas vizinhas? Nesse capitulo desenvolvemos o nosso algoritmo que abrange a solução de problemas de carga espacial bem como de problemas de Laplace. A solução do problema proposto exige que se faça uma junção conveniente entre as duas regiōes que têm como fronteira comum aquela procurada linha de corrente de modo que esta resulte determinada. No cap.VI apresentamos os resultados dos nossos cálculos, precedidos pelos testes que foram realizados do nosso algoritmo. Detalhes sobre a precisão dos cálculos - alguns deles muito peculiares - são também referidos.

No cap.VII, fazemos uma breve avaliação da aplicabilidade ou não da chamada suposição de Deutsch aos casos estudados e, por último, uma sugestão de cálculos futuros encerra este trabalho. 


\section{APITULO}

\section{Equações do Problema de Carga Espacial}

\section{1 - Formas Dimensionais}

Um dos problemas mais simples, formulados no contexto dos fenômenos de carga espacial (e nem por isso sempre fácil de resolver), é o de transporte de carga (ions) unipolar, em regime estacionário, entre dois eletródios mantidos em uma d.d.p constante. O transporte se dá em um meio de permissividade elétrica $\epsilon$, onde os portadores possuem mobilidade $\mu$ e coeficiente de difusão $\kappa$.

As equações básicas que descrevem este problema são:

$$
\begin{gathered}
\nabla \cdot \mathrm{E}=\rho / \epsilon, \\
\nabla \times \mathrm{E}-0, \\
\mathrm{~J}=\mu \rho \mathrm{E}-\kappa \nabla \rho, \\
\nabla \cdot \mathrm{J}=0 .
\end{gathered}
$$

A primeira destas equações é a forma diferencial da lei de Gauss. Nela, como nas demais, $\rho$ é a densidade de carga espacial e $\mathrm{E}$ é a intensidade de campo elétrico, uma combinação do campo de uma fonte externa com a contribuição de campo da própria carga espacial. A eq. (2.2) trata com um campo estático ou que evolui muito lentamente com o tempo no caso quase estacionário e afirma a existência de um campo escalar $\Phi$, o potencial elétrico, tal que

$$
\begin{gathered}
E=-\nabla \Phi, \\
\nabla^{2} \Phi=-\rho / \epsilon .
\end{gathered}
$$

(2.3) define a densidade de corrente, composta pelos termos de fluxo e de difusão, respectivamente, enquanto (2.4) estabelece a continuidade da corrente. 0 coeficiente de difusividade é definido pela relação de Einstein, isto é, 


$$
\kappa=\mu \mathrm{KT} / \mathrm{e}=\mathrm{U}_{\mathrm{D}} \mu
$$

em que $U_{0} \simeq 0.026 \mathrm{~V}$ em temperatura ambiente.

As equações de carga espacial podem ser acopladas em uma única equação em função do potencial elétrico $\Phi$. Para deduzí-la substituem-se as eqs. (2.6) e (2.7) em (2.3), e esta última em (2.4) obtendo-se

$$
\nabla \cdot\left(\nabla^{2} \Phi \nabla \Phi\right)+\mathrm{U}_{\mathrm{D}} \nabla^{2}\left(\nabla^{2} \Phi\right)=0
$$

$\mathrm{Na}$ maioria das situações ${ }^{1}$ o termo de difusão em (2.8) é desprezível em comparação com o de fluxo; em parte porque enquanto $U_{D} \dot{e}$ da ordem de centésimos do volt, a diferença de potencial entre os eletródios é medida em quilovolts. Assim, adotando por hipótese esta simplificação chega-se à equação mais comum do problema de carga espacial, a saber,

$$
\nabla \cdot\left(\nabla^{2} \Phi \nabla \Phi\right)=0
$$

Neste trabalho consideraremos somente o caso $\kappa=0$. Assim, quando nos referirmos às eqs. (2.1) a (2.4) estará implícita esta condição.

\section{2 - Formas Adimensionais}

Para efeito de tratamento numérico é usual e mais cômodo reescrever as equações do problema em termos de grandezas adimensionais. Isto implica na escolha prévia de fatores de escala pelos quais as grandezas originais serão normalizadas. Então, se $\underline{l}$ é uma distância típica entre os eletródios e $\Delta V$ a d.d.p entre eles, uma adimensionalização possivel é obtida quando se fazem as seguintes mudanças de variáveis nas equações anteriores: $(\mathrm{x}, \mathrm{y}, \mathrm{z}) \rightarrow\left(l \mathrm{x}^{\prime}, l \mathrm{y}^{\prime}, l \mathrm{z}^{\prime}\right)$; $\nabla \rightarrow(1 / l) \nabla^{\prime} ; \quad \boldsymbol{\Phi} \rightarrow \Delta \mathrm{V} \boldsymbol{\Phi}^{\prime} ; \mathrm{E} \rightarrow(\Delta \mathrm{V} / l) \mathrm{E}^{\prime} ; \rho \rightarrow\left(\epsilon \Delta \mathrm{V} / l^{2}\right) \rho^{\prime} ; \quad \mathrm{J} \rightarrow\left(\mu \in \Delta \mathrm{V}^{2} / l^{3}\right) \mathrm{J}^{\prime}$

1 Sigmond [5] caracteriza nos seguintes termos as situaçöes em que a corrente de difusão pode ser desprozada:- sejam $z_{0}, t_{1}, t_{2} \bullet z_{3}$ os comprimentos de escala sobre os quals o potencial $\$$ o suas derivadas $\nabla \$, \nabla 2 \$$,

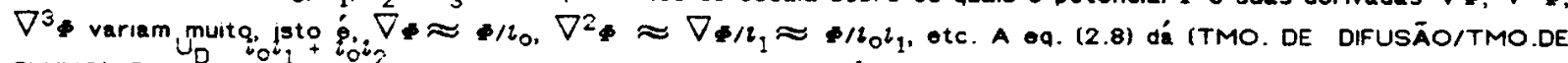

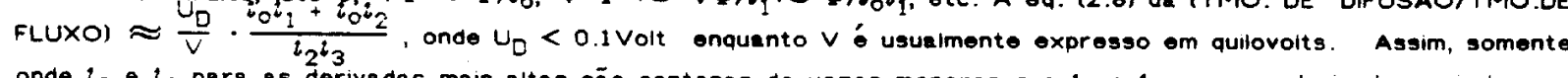
onde $i_{2}$ e $t_{3}$ para as derivadas mais altas são centenas de vezes menores que $i_{0} \theta i_{1}$, para as derivadas mais baixas - termo de difusão será de alguma relevância. 
Para evitar a repetição de equações, é bastante ter em mente que as mudanças se passam como se se tomassem as identidades $\epsilon \equiv 1, \mu \equiv 1$ (e $K \equiv 0$ difusividade desprezivel como foi dito na seção anterior) sobre as equações prévias e nestas, por último, como se as grandezas com dimensão dessem lugar às adimensionais.

Doravante as referências serão feitas tão somente a grandezas e equações adimensionais. E a representação particular do conjunto das equações será feita em função das variáveis sobre as quais se prescrevem diretamente as condições de contorno.

\section{3 - Caracteristicas do Sistema de Equaçōes}

O método hodográfico de Budd \& Wheeler [4] para o tratamento das equações de carga espacial, a ser introduzido no cap.IV, é em parte o método das características. Para a sua compreensão é adequado proceder à determinação das características do sistema de equaçōes da carga espacial bem como de alguma relaçāo sobre elas. Isto também vai propiciar uma base para melhor se entender a escolha de condições de contorno.

Consideremos, pois, reescrever as eqs. (2.1) a (2.4) na forma matricial que se segue, em menção apenas a sistemas bidimensionais:

$$
\begin{gathered}
{\left[\begin{array}{ccc}
1 & 0 & 0 \\
0 & 1 & 0 \\
0 & 0 & \mathrm{E}_{\mathrm{x}}
\end{array}\right] \frac{\partial}{\partial \mathrm{x}}\left[\begin{array}{c}
\mathrm{E}_{\mathrm{x}} \\
\mathrm{E}_{\mathrm{y}} \\
\rho
\end{array}\right]+\left[\begin{array}{ccc}
0 & 1 & 0 \\
-1 & 0 & 0 \\
0 & 0 & \mathrm{E}_{\mathrm{y}}
\end{array}\right] \frac{\partial}{\partial \mathrm{y}}\left[\begin{array}{c}
\mathrm{E}_{\mathrm{x}} \\
\mathrm{E}_{\mathrm{y}} \\
\rho
\end{array}\right]=} \\
=\left[\begin{array}{c}
\rho \\
0 \\
-\rho^{2}
\end{array}\right]
\end{gathered}
$$

$A$ eq. (2.10a) é um caso particular dos sistemas $A(x, y, u) \frac{\partial u}{\partial x}+B(x, y, u) \frac{\partial u}{\partial y}=f(x, y, u)$ e por isso, é um sistema quase linear em $\left\{E_{x}, E_{y}, \rho\right\}$.

Seguindo o procedimento usual, Godounov [6], as caracteristicas de 
(2.10a) são dadas pelas rázes, $\lambda_{i}=d y / d x$, do determinante da matriz

$$
\left[\begin{array}{cc}
A & B \\
d x I & d y I
\end{array}\right]
$$

em que $\underline{A}$ e $\underline{B}$ são, na ordem, as matrizes $3 \times 3$ de (2.10a) e I é a matriz identidade de mesmas dimensões. As ráizes são duas imaginárias, $\lambda_{1,2}= \pm i$, e uma real, $\lambda_{3}-E_{y} / E_{x} \equiv \Phi_{y} / \Phi_{x}$, razão pela qual o sistema das equações de carga espacial, ou resumidamente a eq. (2.9), é do tipo misto: elíptico - configurado na equação de Poisson (2.6) - e hiperbólico.

Certamente o mais importante nesta discussão é observar que as características reais são ortogonais às superfícies equipotenciais, isto é, são as próprias linhas de campo. A equação característica correspondente - a componente hiperbólica do sistema, válida mesmo para o fluxo não estacionário, conforme Sigmond [5] e Leal Ferreira [7] $-\dot{e}^{2}$

$$
d(1 / p)=\frac{d \mathbf{s}}{E}
$$

em que ds é um elemento de comprimento tomado ao longo de uma dada linha de campo e $\mathrm{E}$ é a intensidade de campo na linha considerada.

\section{4 - Condições de Contorno}

As equações reunidas na sec. 2.1 deste capitulo relacionam, entre si (ou podem relacionar), a intensidade de campo, o potencial elétrico e a densidade de carga espacial entre outras grandezas. As condições de contorno a que deverão satisfazer, logicamente se estabelecem pela indicação dos valores destas funções ao longo das fronteiras que delimitam os domínios de solução. Os eletródios, geralmente mantidos a d.d.p constante, fazem a totalidade da fronteira, em alguns dominios uniconexos, ou parte dela nos dominios simplesmente conexos e/ou multiplamente conexos em outros casos. A prescrição do potencial sobre a

${ }^{2}$ para deduzi-ia, substitui-so uma coluna qualquer de $(2.10 b)$ pola coluna do lado direito da eq. (2.10a) devidamente proiongada poias diferonciais $d E_{x}$, $d E_{y}$ edo. Faz-se, om seguida, a determinante desta nova matriz igual a zero. A expressäo resultante : resolvida para $d(1 / \rho)$ om termos de $\left( \pm j a s=\left[(d x)^{2}+(d y)^{2}\right]^{1 / 2}=\left[1+(d y / d x)^{2}\right]^{1 / 2} d x=\right.$ $=\left[E_{x}{ }^{2}+E_{y}{ }^{2}\right]^{1 / 2}\left(d x / E_{x}\right) \equiv\left[E_{x}{ }^{2}+E_{y}{ }^{2}\right]^{1 / 2}\left(d y / E_{y}\right)$. 
fronteira constitui-se então numa condição de contorno natural do problema. Isto é corroborado ainda pela invariância da eq. (2.9) sob uma mudança de escala ou pela adição de uma constante ao potencial $\Phi$ (ou ainda pelo aspecto elíptico daquela equação).

A equação diferencial parcial não linear (2.9), por ser de terceira ordem, exige mais uma condição de contorno além da condição de Dirichlet (e/ou Neumann) sobre o potencial, para que se tenha um problema matemático bem definido. É usual aplicá-la na superficie do eletródio emissor especificando, ou os valores da densidade de carga espacial, como argumentam Felici [8,9] e Atten $[10,11]$, ou os da intensidade de campo, como sugerem Sarma \& Janischewskyj $[12,13]$. A primeira destas alternativas parece óbvia. Vê-se da eq. (2.11a) que se $\rho$ é dada em um extremo de cada linha de campo então, em princípio, fica determinada nos demais pontos de linha por todo o domínio. Ora, as linhas de campo emanam todas do eletródio emissor e este fica, pois, ultimamente, definido por um conjunto de pontos extremos. Com $r_{e}$ e $\rho_{e}$ na fronteira emissora e $r$ no ponto de linha, então

$$
\frac{1}{\rho}=\frac{1}{\rho_{e}}+\int_{r_{e}}^{r} \frac{d s}{E}
$$

Na segunda alternativa a condição de contorno é mais apropriada a um emissor em que a carga é produzida por descarga corona. Trata-se da intensidade de campo harmônico (solução da equação de Laplace) quando a tensão atinge o valor de disparo de corona, pois, postula-se que o papel da corona é manter constante a intensidade do campo nas suas vizinhanças imediatas, independentemente do crescimento da voltagem acima do nivel de disparo.

Revendo a discussão em alguns diferentes contextos, Budd \& Wheeler [4] têm constatado a inexistência de uma condição de contorno universal. Assumem que existe uma dada relação entre intensidade de campo e carga espacial no emissor da forma

$$
\mathrm{F}(\theta,|\mathrm{E}|, p)=0
$$


onde $F$, a que se referem como "lei de injeção", é uma função apropriada à situação considerada e $\theta$ é uma variável em termos da qual se parametriza a superfície de emissão.

\section{5 - Soluções Exatas}

Há poucas soluções algébricas exatas para o problema de carga espacial. A mais simples é a solução unidimensional descrevendo o fluxo entre dois eletródios planos. Para uso posterior nos Caps.III e IV, vamos explicitá-la detalhadamente. Com a distância y tomada ao longo da direção transversal aos eletródios, estando o emissor em $y=0$ e o coletor em $y=1$, tem-se,

$$
\begin{aligned}
& \rho(y)=\rho_{\mathrm{O}}\left[1+\frac{2 \rho_{\mathrm{O}}}{\mathrm{E}_{\mathrm{O}}} \mathrm{y}\right]^{-1 / 2}, \quad 0 \leq \mathrm{y} \leq 1 ; \\
& E(y)=E_{o}\left[1+\frac{2 \rho_{O}}{E_{o}} y\right]^{1 / 2} \hat{\mathbf{y}} ; \\
& \Phi(y)=1-\frac{E_{o}^{2}}{3 \rho_{0}}\left[\left(1+\frac{2 \rho_{0}}{E_{o}} y\right)^{3 / 2}-1\right] ; \\
& E_{O}^{3}+\left(2 \rho_{O}-1\right) E_{O}^{2}+\frac{4}{3} \rho_{O}^{2} E_{O}-\frac{3}{2} \rho_{O}=0 .
\end{aligned}
$$

A última relação é necessária para que a d.d.p entre os eletródios seja $\Delta \Phi=1$.

As soluções em duas e três dimensões mais frequentemente mencionadas têm fronteiras consistindo de cilindros coaxiais e de esferas concêntricas com densidade de carga espacial uniforme no emissor. São facilmente dedutiveis bastando dizer que a eq. (2.9) é trivialmente separável tanto em coordenadas polares cilindricas quanto em coordenadas esféricas. Isto equivale a dizer que a presença de carga espacial nestas geometrias não afeta a direção das linhas do campo harmônico correspondente ou que as superfícies equipotenciais são congruentes às laplacianas, podendo apenas ser distribuidas de maneira diferente. Esta afirmação constitui a essência da chamada "suposição de Deutsch", a qual será desenvolvida na sec. 2.6 .

Smith [14], usando métodos da teoria de variáveis complexas, 
determinou o conjunto completo das soluções exatas para as quais a suposição de Deutsch é correta. Assim o fêz, motivado na frequente asserção de que a introdução de carga espacial afeta fortemente a direção do campo; e as soluçōes até então formuladas estavam em grande parte baseadas exatamente no contrário.

O conjunto de Smith está descrito por duas fórmulas canônicas envolvendo vários parâmetros livres. Uma delas,

$$
z=k \int \exp \left(n w+\frac{1}{4} b w^{2}\right) d w
$$

é uma transformação conforme representando as coordenadas espaciais $(x, y)$ em termos do potencial e fluxo harmônicos $(\phi, \psi)$, tais que $z=x+i$ y e $w=\phi+i \downarrow . k e$ n são constantes complexas e b é uma constante real. Em (2.14a) está implícita mais uma constante de integração. A outra fórmula,

$$
\Phi=f(\phi)=l \int d \phi\left\{\int \exp \left(a t+\frac{1}{2} b t^{2}\right) d t\right\}^{1 / 2}
$$

define o potencial de carga espacial, $\Phi$, em função unicamente do potencial laplaciano $\phi . l$ é uma constante, real ou imaginário puro, dependendo do sinal do radicando; $a=2 \Re(n)$ e $t$ é uma variável (real) de integração. (2.14b) prevê ainda duas constantes de integração.

Com $m-\Im(n)$, a intensidade de campo, a densidade de carga espacial e - ângulo entre a direção do campo e o eixo x no plano $z$ são, respectivamente,

$$
\begin{gathered}
E=\frac{l}{|k|} \exp \left(m \psi-\frac{1}{2} a \phi-\frac{1}{4} b\left(\phi^{2}-\psi^{2}\right)\right)\left\{\int^{\phi} \exp \left(a t+\frac{1}{2} b t^{2}\right) d t\right\}^{1 / 2}, \\
\nabla^{2} \Phi=\frac{l}{2|k|^{2}} \exp \left(2 m \psi+\frac{1}{2} b \psi^{2}\right)\left\{\int^{\phi} \exp \left(a t+\frac{1}{2} b t^{2}\right) d t\right\}^{-1 / 2}, \\
\arg (d z / d w)=\arg (k)+m \phi+\frac{1}{2} a \psi+\frac{1}{2} b \phi \psi .
\end{gathered}
$$

A dedução de todas as soluçōes se faz pelas escolhas possiveis dos parâmetros livres. Assim, qualitativamente, $\underline{n-b-0}$ dá a solução unidimensional; $\Im(n)=b=0, a \neq 0$ produz a já mencionada solução bidimensional com equipotenciais em círculos concêntricos. Menos comum é a solução de corrente girante obtida pela escolha $a-b-0, \Im(n)-m \neq 0$. Neste caso as linhas de campo 
$(\Im(w)=c t e$.$) são circulos e as equipotenciais (\Re(w)=c t e$.$) são linhas retas$ irradiando-se de um ponto (o centro dos círculos). Mais três soluções são possiveis com dedução bem mais elaborada. A de Varley [15] que corresponde a $b=0, \Re(n) \neq 0, \Im(n) \neq 0$ e possui equipotenciais em espirais e linhas de campo em espirais que giram em sentido contrário. Por último vêm as duas novas soluções de Smith que correspondem às duas possibilidades $b \neq 0 \quad(b>0$ e $b<0)$. A transformação conforme envolve a função erro $(z=\operatorname{erf}(w))$, de modo que as equipotenciais e linhas de campo destas soluções não têm uma forma geométrica comum.

Budd \& Wheeler [16] demonstraram que as soluções representadas pelas eqs. (2.14a) e (2.14b) são as soluções separáveis das equações que resultam quando sua transformacão hodográfica (a ser descrita em detalhes no cap.IV) é operada na equação de carga espacial, eq. (2.9). As coordenadas de separação são como antes, o potencial e $\circ$ fluxo harmônicos $(\phi, \psi)$.

\subsection{1 - Emprego de Soluçōes Exatas}

O exposto nesta seção objetiva apenas exemplificar algumas aplicações de soluções exatas das equações de carga espacial em problemas de domínios diferentes. Não há a intenção de exaurir todas as possibilidades nem de descrever em detalhes os exemplos citados.

As soluções exatas são frequentemente empregadas para testar, por comparação direta, a precisão de métodos numéricos visando a resolução numérica de problemas em outras configurações. Eventualmente podem representar o limite assintótico da solução de um problema particular, bastando que a geometria e condições de contorno, no limite, se aproximem daquelas da solução exata considerada.

Uma das soluções congruentes com $b \neq 0(b<0$ e $n=0$ em $(2.14 a))$ pode ser posta em uma configuração de equipotenciais e linhas que possui marcada semelhança com o problema prático do sistema linha-terra (ou fio-plano aterrado) 
em regime de corona. Smith explicitou a caracteristica voltagem-corrente desta soluçãc em termos de funções elementares e em referôncia direta à geometria linha-terra:

$$
V=\frac{V_{0}}{\chi_{0}} \int_{0}^{\chi_{0}}\left\{1+\frac{2}{\pi} \frac{i_{A}}{\epsilon \mu}\left(\frac{L \chi_{0}}{V_{0}}\right)^{2}\left(\operatorname{erf}\left(\chi_{0}\right)-\operatorname{erf}(t)\right)\right\}^{1 / 2} d t
$$

onde $\chi_{0}$ é a solução positiva de

$$
\frac{2}{\pi^{1 / 2}} \frac{\exp \left(-\frac{1}{2} \chi_{0}^{2}\right)}{\chi_{0}}-\frac{r}{L}=0
$$

$\underline{r}$ é o raio da linha (fio condutor), $\underline{L}$ é a altura ao plano aterrado e $V_{O}$ é a tensão de disparo de corona. A concordância de (2.15a) com a experiência é satisfatória para todos os conjuntos de dados por ele considerados, notadamente se se referem a um fio de superficie polida.

\section{6 - Soluçōes Aproximadas ${ }^{3}$}

Nesta seção, a discussão será desenvolvida em torno de algumas das aproximaçōes que estão embasadas na suposição de Deutsch. Apresentam-se, por último, breves comentários sobre uns poucos procedimentos numéricos destinados ao problema.

As aproximações analiticas compõem parte da literatura preliminar sobre o assunto e constituem o conteúdo dos trabalhos de Deutsch [17], Popkov $[18,19,20]$ e os mais recentes de Sigmond [21] e Walsh, Gallo \& Lama [22] entre outros. Invariavelmente tratam com a descarga corona nas geometrias (de grande interesse prático) fio-plano e ponta(hiperbolóide)-plano. Os dados experimentais são interpretados por comparação direta com as características voltagem-corrente calculadas e/ou com as distribuições angulares ${ }^{4}$ da densidade de corrente sobre o

\footnotetext{
30 termo "aproximadas" : usado para designar eproximaçōos a probloma (do carga espacial) om que não ocorrem dominios em que sejam de fato soluçöes oxatas das equações de carga espacial. Excluem-so do contexto, portento, as aplicaçōes mencionadas na seçāo anterior.

4Uma distribuçăo angular oxpressa J sobre o plano colotor como uma função do ángulo compreendido entre o plano e/ou eixo de simetria dos sistemas com a reta que val de um ponto mais avançado do omissor ao ponto de observaçāo no coletor.
} 
plano coletor.

O ponto de partida para o cálculo é a relação entre a intensidado do campo com carga espacial, $E$, e a do campo laplaciano correspondente, $E_{L}$, a saber,

$$
\mathbf{E}=\boldsymbol{\theta} \mathbf{E}_{\mathbf{L}}
$$

em que $\theta$ é uma função escalar das coordenadas. Seguindo-se a exposição de Sigmond [5], as equações do problema assumem as seguintes formas quando se incorpora a suposicão de Deutsch, eq. (2.16a):

- Equação de Poisson, eq. (2.6):

$$
\nabla \cdot E-\nabla \theta \cdot E_{L}+\theta \nabla \cdot E_{L}=\nabla \theta \cdot E_{L}=E_{L} \frac{\partial \theta}{\partial s}=\frac{\rho}{\epsilon_{0}} ;
$$

$S$ é a coordenada de posição ao longo das linhas de campo.

$$
-\nabla \times E-0 \text {, eq. (2.2): }
$$

$$
\nabla \times E=\nabla \theta \times E_{L}+\theta \nabla \times E_{L}=E_{L} \times \nabla \theta=0 ;
$$

então $\nabla \theta$ é paralelo a $E_{L}$, ○ que significa $\theta=\theta(\phi)$-cte. ao longo das superfícies equipotenciais laplacianas $(\phi-c t e$.$) .$

- Conservagão da corrente, eqs. (2.4) e (2.3):

$$
\nabla \cdot J=\nabla \cdot\left(\mu \rho \theta E_{L}\right)=\nabla(\mu \rho \theta) \cdot E_{L}+\mu \rho \theta \nabla \cdot E_{L}=E_{L} \frac{\partial(\mu \rho \theta)}{\partial s}=0 \text {, }
$$

donde se conclui que

$$
\mu \rho \theta-J / E_{L}=C(\tau),
$$

com as coordenadas perpendiculares a $\underline{\mathbf{s}}$ representadas por $\underline{\tau}$.

Sobre uma linha de campo ds- $\mu$ Edt, em que dt é o tempo infinitesimal durante o qual o portador percorre a distância ds. Em termos do tempo de trânsito dos portadores, $t=\int(1 / \mu E) d s$, a eq. (2.11a) pode ser integrada para dar, em grandezas dimensionais,

$$
\rho(t)=\frac{\rho_{e}}{\left[1+\left(\mu \rho_{e} t / \epsilon\right)\right]}, \quad \rho_{e}=\rho(t=0) .
$$

Se a densidade de carga $\rho_{O}$ torna-se muito grande no eletródio emissor, $\rho(t) \rightarrow(\epsilon / \mu t)$. Por outro lado se $\rho_{0} \ll(\epsilon / \mu t)$, então $\rho(t) \approx \rho_{O}$. 
A) Sobre uma superficie equipotencial laplaciana qualquer $(\theta-\theta(\phi-c t e)$.$) a densidade de carga espacial é, por (2.16 \mathrm{~b})$,

$$
\rho=\epsilon E_{L} \frac{\partial \theta}{\partial s}=\epsilon \frac{d \theta}{d \phi} \frac{\partial \phi}{\partial s} E_{L}=-\epsilon E_{L}^{2} \frac{d \theta}{d \phi}
$$

o que substituído em (2.16d) produz

$$
\begin{aligned}
J=\mu \rho \theta E_{L} & =-\mu \epsilon\left(\theta \frac{d \theta}{d \phi}\right) E_{L}^{3}, \quad \text { ou, } \\
J(\tau) & \propto E_{L}^{3}(\tau) .
\end{aligned}
$$

Este é o resultado de Walsh-Gallo-Lama.

B) Combinando as eqs. (2.16b) e (2.16d) encontra-se

$$
\frac{\partial \theta}{\partial s}=\frac{\rho}{\epsilon \mathrm{E}_{\mathrm{L}}}=\frac{\mathrm{C}}{\mu \epsilon \theta \mathrm{E}_{\mathrm{L}}} .
$$

Deutsch tomou neste resultado $\theta=1$, aproximação que corresponde a uma pequena corrente (ou pequena carga espacial injetada), e integrou (2.16g) para obter

$$
\theta=\frac{C}{\epsilon} \int_{e} \frac{d s}{\mu E_{L}}+\theta_{e}=\frac{C}{\epsilon} t_{L}+\theta_{e} .
$$

A d.d.p entre o eletródio emissor (e), ao potencial V, e o coletor (c), aterrado, é dada por

$$
V=\int_{e}^{c} E d s=\int_{e}^{c} \theta E_{L} d s=\frac{C}{\epsilon} \int_{e}^{c} E_{L} t_{L} d s+\theta_{e} V
$$

ou, usando outra vez (2.16d),

$$
V=\frac{J_{C}}{\epsilon E_{L c}} \int_{e}^{c} E_{L} t_{L} d s+\theta_{e} V
$$

Para determinar $\theta_{e}$ Deutsch supôs a intensidade de campo sobre o emissor, $E_{e}$, igual à do campo sobre a fronteira da região de ionização, $E_{o}\left(\tau_{e}\right)$, no limiar de corona. Tomou então $\theta_{e}=E_{e} / E_{L}=v_{0}\left(\tau_{e}\right) / V$, de modo que o nivel mais baixo de $v_{0}$ sobre a superfícies da corona é a tensão de disparo, $V_{0}$. Substituindo $\theta_{e}$ em 
(2.16h) e resolvendo para $J_{c}$, encontra-se a fórmula de Deutsch,

$$
J_{C}=\frac{\epsilon E_{L C}}{\int_{0}^{c} E_{L} t_{L} d s}\left(V-v_{0}\right)
$$

ou, ainda, com $t_{L}=\int_{0}\left(1 / \mu E_{L}\right) d s, E_{L}=-d \phi / d s, u=\phi / V$ e $g_{L}=E_{L} / V$,

$$
J_{C}=\frac{\mu \epsilon E_{L C}\left(V-v_{0}\right)}{\int_{0}^{c}\left(\phi / E_{L}^{2}\right) d \phi}=\frac{\mu \epsilon g_{L C}}{\int_{0}^{1}\left(u / g_{L}^{2}\right) d u} V\left(V-v_{0}\right)
$$

C) Popkov evitou a aproximação de baixa corrente $(\theta=1)$ usada por Deutsch e integrou a eq. (2.16g) para $\theta$, encontrando, com $t_{L}$ definido como antes,

$$
\theta \frac{\partial \theta}{\partial s}=\frac{C}{\mu \epsilon E_{L}} \Rightarrow \theta=\sqrt{\frac{2 C t_{L}}{\epsilon}+\theta_{e}^{2}}
$$

Também fez $\theta_{\mathrm{e}}=v_{\mathrm{o}}$ /V. A d.d.p entre os eletródios é agora,

$$
V=\int_{e}^{c} E d s=\int_{e}^{c} E \sqrt{\frac{2 C t_{L}}{\epsilon}+\left(\frac{U_{O}}{V}\right)^{2}} d s
$$

Outra vez com $C=J_{C} / E_{L C}, t_{L}=\int_{0}\left(1 / \mu E_{L}\right) d s, E_{L}=-d \phi / d s$,

$$
V=\int_{0}^{V} d \phi \sqrt{\frac{2 J_{C}}{\mu \epsilon E_{L C}} \int_{\phi}^{V}\left(1 / E_{L}\right)^{2} d \phi^{\prime}+\left(v_{o} / V\right)^{2}} .
$$

As mudanças de variáveis $u=\phi / V, \quad g_{L}=E_{L} / V$ e $M_{C}=2 J_{C} /\left(\mu \epsilon g_{L C} v_{O}^{2}\right)$ permitem reescrever a eq. (2.16l) na forma adimensional que segue, conhecida por fórmula "L" de Popkov:

$$
\frac{V}{v_{0}}=\int_{0}^{1} d u \sqrt{M_{c} \int_{u}^{1}\left(1 / g_{L}{ }^{2}\right) d u^{\prime}+1}
$$

Para evitar as dificuldades de cálculo inerentes a $(2.16 \mathrm{~m})$, Popkov deduziu também uma outra fórmula para determinar $J\left(\tau_{C}\right)$ em correspondência a cada $V$ dado. Do fato de que $\theta=\theta(\phi)$, conforme (2.16c), concluiu que sobre a superficie do coletor $\left(\tau_{c}\right)$, 


$$
C T_{L}=\left(J_{c} / E_{L C}\right) \int_{e}^{c} \frac{d s}{\mu E_{L}}=c t e . \quad \Rightarrow \quad J_{c} \propto \frac{E_{L}}{T_{L}},
$$

do que resulta imediatamente a fórmula " $T$ " de Popkov,

$$
J_{C} / I=\left(E_{L C} / T_{L}\right) / \int_{\tau_{c}}\left(E_{L C} / T_{L}\right) d^{2} \tau
$$

D) A distribuição de densidade de corrente de Sigmond [21] é válida para o fluxo em carga espacial saturada e estabelece um limite superior desta densidade para cada voltagem dada, V. Assim, tomando $\rho_{e} \gg(\epsilon / \mu t)$ em (2.16e) obtém-se a aproximação $\rho \approx \epsilon / \mu t$ ou $\rho_{\mathrm{c}} \approx \epsilon / \mu \mathrm{T}$ sobre o coletor. Para uma linha de campo de comprimento $L$, Sigmond considerou $E \approx V / L$ e $T \approx L / \mu E \approx L^{2} / \mu V$, com o que chega finalmente a sua fórmula,

$$
\mathrm{J}_{\mathrm{c}}=\mu \rho_{\mathrm{c}} \mathrm{E} \approx \mu \epsilon \mathrm{V}^{2} / \mathrm{L}^{3}
$$

Para calcular L em (2.160), Sigmond recorreu à suposição de Deutsch, isto é, tomou o padrão de linhas laplacianas. O seu resultado, em que $J_{c} \propto E / T$, assemelha-se à fórmula $T$ de Popkov, $J_{c} \propto E_{L} / T_{L}$. Todavia a diferença entre $E_{L}$ e $E=V / L$ as torna diferentes.

As soluções de campo laplaciano para as geometrias fio-plano e ponta (hiperbolóide)-plano são há muito conhecidas de modo que podem ser usadas para reescrever as distribuiçōes de corrente aqui apresentadas, em forma de distribuições angulares. Isto está feito extensa e detalhadamente no artigo de revisão de Sigmond [5], onde também se encontram comparações gráficas (Figuras 3 e 4) das várias curvas teóricas com as experimentais e algumas poucas exatas calculadas por métodos numéricos (elementos finitos).

No caso das geometrias fio-plano e ponta-plano, concordância mais satisfatória e consistente é encontrada entre os dados experimentais e as distribuições de densidade de corrente ditas do tipo "L" $L$ (distribuições de

\footnotetext{
"Por causa ô sua dedução 20 longo das linhas de campo essas distribuiçõos são rotuladas por " $L "$. As
} deduzidas transversalmente ìs linhas de campo são ditas do tipo "T". 
Deutsch, L de Popkov, de Sigmond e outras), principalmente nos pontos de coletor (plano aterrado) mais próximos do plano e eixo de simetria desses sistemas de corona. Entretanto tal concordância parece estar limitada pela própria essência da suposiçao de Deutsch, porque, como afirma Sigmond [5], "fórmulas calculando a densidade de corrente das condições laplacianas em um ponto local do espaço da corona não poderiam refletir a natureza global das interações da carga espacial".

Os procedimentos numéricos são, talvez, os métodos mais eficientes de solução do problema de carga espacial. Dão resultados de primeiros princípios e somente estão sujeitos às limitações das técnicas numéricas particulares empregadas.

O procedimento iterativo de Atten [11], muito citado na literatura, consta dos seguintes passos: (1) resolve a eq. (2.6), isto é, $\nabla^{2} \Phi=-\rho$, usando uma escolha adequada de $\rho$ (usualmente $\rho-0$ ) e os valores do potencial nos eletródios como condição de contorno; (2) a solução determinada para $\Phi$ é então substituída na equação da continuidade do fluxo, reescrita por conveniência na forma $\boldsymbol{\nabla} \boldsymbol{\Phi} \cdot \boldsymbol{\nabla}(1 / \rho)=-1$, e uma nova distribuição de carga $\rho$ é obtida. Os valores de $\rho$ no eletródio emissor são a condição de contorno. Com a nova distribuição $\rho, 0$ processo retorna ao passo (1) e segue até a convergência. O procedimento foi empregado às geometrias já referidas nesta seção. E a principal dificuldade é determinar $\rho$ sobre a região de ionização, isto é, dar a condição de contorno em $\rho$.

Janischewshyj et.al. [23] e Takuma et.al. [24] recorreram a técnicas de elementos finitos como método de tratamento das equações de carga espacial.

A mais recente novidade dentre os enfoques numéricos do problema de carga espacial é o método hodográgico de Budd \& Wheeler [4]. O cap.IV será dedicado à sua descrição. Por enquanto è bastante dizer que consiste essencialmente em inverter o sistema de coordenadas e resolver as equações para a densidade de carga e coordenadas de posição sobre uma malha composta por equipotenciais e linhas de fluxo. 


\title{
C AP ITULO III
}

\section{Solução das Equações de Carga Espacial - uma Aproximação em Séries de Taylor Truncadas}

\section{1 - Introdução}

\begin{abstract}
Neste capitulo esboçaremos uma solução das equações de carga espacial na forma de séries de Taylor truncadas nos primeiros termos. Não pretendemos abranger geometrias e condições de contorno gerais, senão tratar, inicialmente, com um problema extremamente simples como o de um fluxo estacionário de carga espacial unipolar a partir de uma equipotencial plana infinita, então suposta a fonte da carga, com densidade espacial de carga e intensidade de campo prescritos. Para maior clareza, suponhamos que o fluxo se estenda em todo o semiplano superior em um sistema de coordenadas cartesianas onde a fonte está representada pelo eixo x (ou plano $x z$ ), conforme Fig. -3.1 .
\end{abstract}

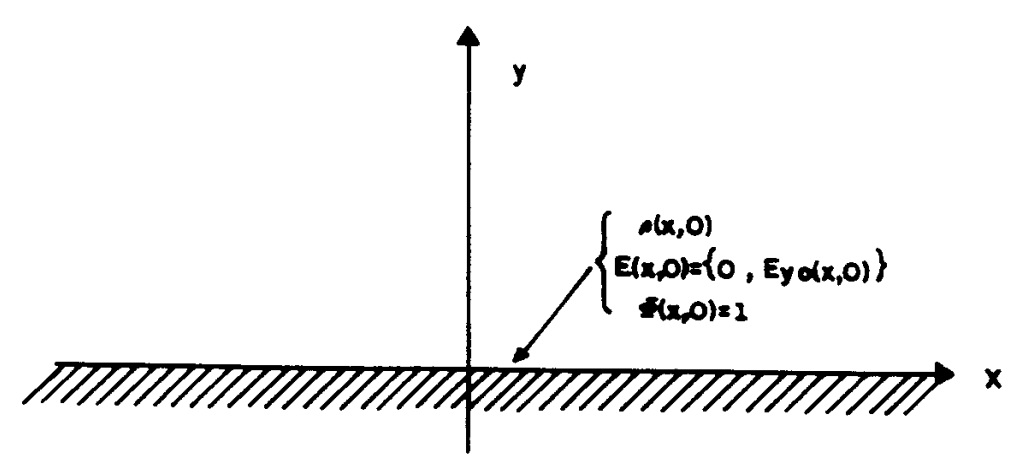

Fig.-3.1 Diagrama do domínio de solução.

Posteriormente, nas secões (3.3) e (3.4), faremos uma aplicação da solução para calcular equipotenciais no dominio definido por um par de eletródios planos paralelos, na situação não usual de uma densidade de carga não uniforme no emissor. O teste de validade é a comparacão com equipotenciais correspondentes calculadas pelo método hodográfico. 
Preliminarmente a densidade $\rho(x, y)$ e as componentes do campo, $E_{x}(x, y)$ e $E_{y}(x, y)$, são expandidas em termos de potências de $x$ e $y$. Os coeficientes são então determinados por substituição das expansões nas eqs. (2.1), (2.2) e (2.4) ou, para sermos mais explicitos, nas versões correspondentes

$$
\begin{gathered}
\frac{\partial E_{x}}{\partial x}+\frac{\partial E_{y}}{\partial y}=\rho, \\
\frac{\partial E_{y}}{\partial x}=\frac{\partial E_{x}}{\partial y}, \\
\frac{\partial \rho}{\partial x} E_{x}+\frac{\partial \rho}{\partial y} E_{y}=-\rho^{2},
\end{gathered}
$$

e exigindo que estas sejam satisfeitas pelo menos em uma aproximação de $2^{a}$. ordem. Obviamente a determinação se dá em uma relação formal entre coeficientes por um lado e as condições de contorno por outro, como veremos na próxima seção.

\section{2 - Determinaçāo de Coeficientes}

Tomando a forma usual da série de Taylor de uma função de duas variáveis em torno do ponto $\left(x_{0}, y_{0}\right)$ e tendo em mente a notação simplificada $\left(\mathrm{x}-\mathrm{x}_{0}\right) \rightarrow \mathrm{x},\left(\mathrm{y}-\mathrm{y}_{0}\right) \rightarrow \mathrm{y}, \mathrm{E}_{\mathrm{x}}\left(\mathrm{x}_{0}, \mathrm{y}_{0}\right) \rightarrow \mathrm{E}_{\mathrm{x} O}, \mathrm{E}_{\mathrm{y}}\left(\mathrm{x}_{0}, \mathrm{y}_{0}\right) \rightarrow \mathrm{E}_{\mathrm{y}} \mathrm{e} \rho\left(\mathrm{x}_{0}, \mathrm{y}_{0}\right) \rightarrow \rho_{0}$, podemos escrever

$$
\begin{gathered}
E_{x}(x, y)=E_{x o}+a_{1} x+a_{2} y+a_{3} x^{2}+a_{4} x y+a_{5} y^{2}+\sigma(3) \\
E_{y}(x, y)=E_{y o}+b_{1} x+b_{2} y+b_{3} x^{2}+b_{4} x y+b_{5} y^{2}+\sigma(3) \\
\rho(x, y)=\rho_{0}+c_{1} x+c_{2} y+c_{3} x^{2}+c_{4} x y+c_{5} y^{2}+\sigma(3)
\end{gathered}
$$

em que os $a_{i}, b_{i}$ e $c_{i}(i=1,2, \ldots, 5)$ são funções de $E_{x o}, E_{y o}, \rho_{O}$ e das derivadas em $x$, no ponto $\left(x_{0}, y_{0}\right)$, de $E_{x}, E_{y}$ e $\rho$. Para que as expansões (3.2) a (3.4) sejam compativeis com uma equipotencial em $y=y_{0}=0$, é necessário que

$$
E_{x_{0}}\left(x_{0}, 0\right)-a_{1}\left(x_{0}, 0\right)-a_{3}\left(x_{0}, 0\right)=0, \quad \forall x_{0}
$$

Alguns dos coeficientes seguem diretamente da fórmula de Taylor, a saber, 


$$
\begin{aligned}
& a_{2 i-1}=\frac{1}{i !} \frac{\partial^{(i)} E_{x}\left(x_{O}, y_{O}\right)}{\partial x^{(i)}}, \\
& b_{2 i-1}=\frac{1}{i !} \frac{\partial^{(i)} E_{y}\left(x_{O}, y_{O}\right)}{\partial x^{(i)}}, \\
& c_{2 i-1}=\frac{1}{i !} \frac{\partial^{(i)}\left(x_{O}, y_{O}\right)}{\partial x^{(i)}}, \quad i=1,2 .
\end{aligned}
$$

Os demais são obtidos por substituição direta das séries (3.2) a (3.4) nas eqs. de carga espacial (3.1a) a (3.1c):

$$
\begin{aligned}
& a_{2}=b_{1}, \\
& a_{4}=2 b_{3} \\
& a_{5}=\frac{1}{2} c_{1}-c_{3} \\
& b_{2}=\rho_{0}-a_{1} \\
& b_{4}=c_{1}-2 a_{3} \\
& b_{5}=-b_{3}+\frac{1}{2} c_{2} \\
& c_{2}=-\left(\frac{\rho_{0}^{2}}{E_{y 0}}+\frac{E_{x O}}{E_{y 0}} c_{1}\right) \\
& c_{4}=-\left(\frac{2 \rho_{0}}{E_{y o}} c_{1}+\frac{1}{E_{y o}} b_{1} c_{2}+\frac{1}{E_{y o}} a_{1} c_{1}+\frac{2 E_{x o}}{E_{y o}} c_{3}\right) ; \\
& c_{5}=\left(\frac{1}{2 E_{\mathrm{yO}}} a_{1}-\frac{3 \rho_{0}}{2 E_{\mathrm{yO}}}\right) c_{2}-\frac{1}{2 E_{\mathrm{yo}}} b_{1} c_{1}-\frac{E_{\mathrm{XO}}}{2 E_{\mathrm{yo}}} c_{4} .
\end{aligned}
$$

Estes coeficientes estão todos relacionados com $a_{2 i-1}, b_{2 i-1}$ e $c_{2 i-1}, i-1,2$, numa afirmação de que se a densidade de carga $\rho_{0}$ e a componente de campo Eyo são dados na fonte, como supostamente 0 são, as grandezas de campo ficam completamente determinadas, em 2* ordem, em todos os ponto do dominio. A 2: ordem aqui é apenas uma opção. Poder-se-ia tomar uma ordem mais alta e ainda assim todas as deduçōes seriam exequiveis apesar do algebrismo crescer. 


\section{3 - Sistema de Dois Eletródios Planos}

A geometria da familia de equipotenciais representadas pela solução em séries, eqs. (3.2) a (3.11), é completamente dominada pela natureza das condições de contorno impostas na equipotencial $\Phi(x, 0)-1$ da Fig.-3.1. Para exemplificar, suponhamos que, em todo ponto $x$ do eletródio emissor, $\rho(x, 0)-\rho_{0}=c$ te. e $E_{y}(x, 0)=E_{o}-c t e$. Como consequência todos os coeficientes se anulam, com exceção apenas de $b_{2}, b_{5}, c_{2}$ e $c_{5}$ que têm os valores

$$
b_{2}=\rho_{0} ; \quad b_{5}=-\frac{1}{2} \frac{\rho_{0}^{2}}{E_{0}} ; \quad c_{2}=-\frac{\rho_{0}^{2}}{E_{0}} ; \quad c_{5}=\frac{3}{2} \frac{\rho_{0}^{3}}{E_{0}^{2}}
$$

No presente caso as expansões se resumem a

$$
\begin{gathered}
\rho(y)=\rho_{o}-\frac{\rho_{o}^{2}}{E_{o}} y+\frac{3}{2} \frac{\rho_{o}^{3}}{E_{o}^{2}} y^{2}+\sigma(3), \\
E_{y}(y)=E_{o}+\rho_{o} y-\frac{1}{2} \frac{\rho_{o}^{2}}{E_{o}} y^{2}+\sigma(3) .
\end{gathered}
$$

Por simples inspeção podemos verificar que elas encerram os três primeiros termos do desenvolvimento binomial da solução de equipotenciais planas das eqs. (2.13a,b). Assim as expansōes representam, neste caso, uma aproximação à solução unidimensional, válida em $0 \leq y \leq 1$ e sob a condição $\left(2 \rho_{o} / E_{o}\right)<1$.

Para tratar o sistema de dois eletródios planos, paralelos, em condição não trivial de injeção, admitiremos em principio que, para algumas densidades particulares dadas, $\rho(x, 0)$, haverá pelo menos um campo de injeção, $E_{y}(x, 0)$, sob - qual a solução em séries apresenta mais uma equipotencial plana, digamos $\Phi(\mathrm{x}, 1)=0$, além daquela já existente $\Phi(\mathrm{x}, 0)=1$. Estas equipotenciais acomodariam então os dois eletródios do sistema e as séries resultantes aproximariam a solução do problema (de carga espacial) nessa geometria.

Suponhamos que a carga espacial no emissor tenha a seguinte distribuição

$$
\rho(x, 0)=\rho_{0}\{2[1-\tanh (n x)]+\alpha\}
$$


onde $\rho_{O}, \mathrm{n}$ e $\alpha$ são parâmetros constantes, todos positivos. Esta particular escolha de $\rho(x, 0)$ é muito conveniente às nossas finalidades, a começar pela definição da condição de contorno $E_{y}(x, 0)$. Em $x \leq-(6 / n)$ e $x \geq(6 / n)^{1}$ a densidade de carga injetada assume os valores assintóticos $(4+\alpha) \rho_{0}$ e $\alpha \rho_{0}$, respectivamente, conforme a eq. (3.14). Ora, se os valores correspondentes do campo de emissão forem também constantes, $E_{O}$ e $E_{O}^{\prime}$ por definição, fica assegurado que a solução do problema tem equipotenciais todas planas nos subdominios $x \leq-(6 / n)$ e $x \geq(6 / n)$. Os valores $E_{o}$ e $E_{o}^{\prime}$ serão determinados a partir das constantes $\rho_{o}$ e $\alpha$ (eq. (3.14)) de modo que a equipotencial $\Phi=0$ coincida completamente com o plano $y=1$, nas condições da Fig. -3.2 .

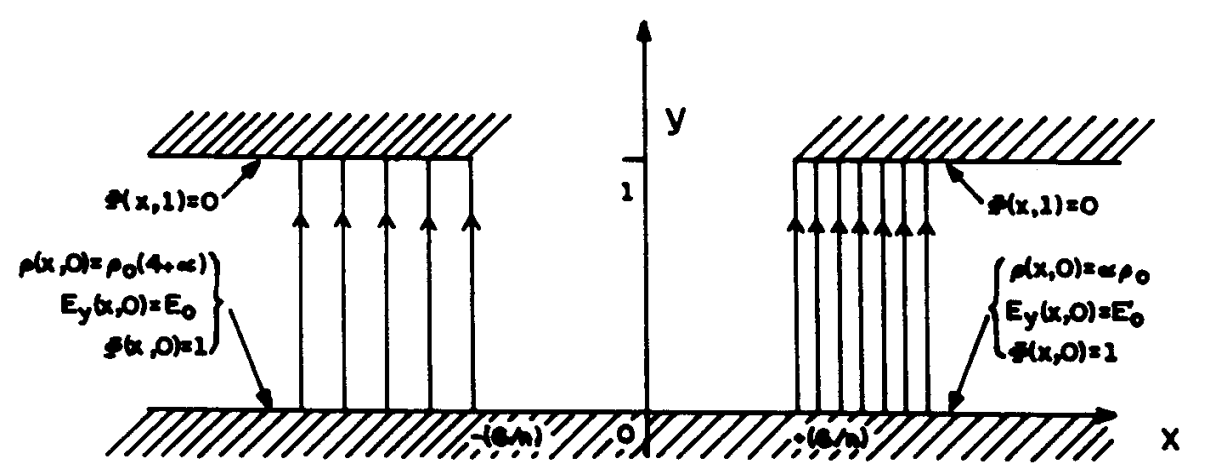

Fig.-3.2 Condições de contorno na regiāo assintótica

As distribuições de campo nas regiōes assintóticas são, pela eq. (2.13b) e no sentido da Fig. -3.2 ,

$$
\begin{aligned}
& E(x, y)=E_{o}\left\{1+\frac{2\left[\rho_{o}(4+\alpha)\right]}{E_{o}} y\right\}^{1 / 2}, \quad x \leq-(6 / n) \\
& E^{\prime}(x, y)=E_{o}^{\prime}\left\{1+\frac{2\left(\rho_{o} \alpha\right)}{E_{o}} y\right\}^{1 / 2}, \quad x \geq(6 / n)
\end{aligned}
$$

Integrando $E(x, y)$ e $E^{\prime}(x, y)$ de $y=0$ a $y=1$ e lembrando que os resultados assim obtidos são iguais à d.d.p $\Delta \Phi-\Phi(x, 0)-\Phi(x, 1)=1$, obtém-se relações do tipo $(2.13 d)$, is to $\dot{e}$

$$
\begin{aligned}
& E_{O}^{3}+\left\{2\left[\rho_{O}(4+\alpha)\right]-1\right\} E_{o}^{2}+\frac{4}{3}\left[\rho_{o}(4+\alpha)\right]^{2} E_{o}-\frac{3}{2}\left[\rho_{O}(4+\alpha)\right]=0, \\
& E_{o}^{3}+\left[2\left(\rho_{o} \alpha\right)-1\right] E_{o}^{, 2}+\frac{4}{3}\left(\rho_{o} \alpha\right)^{2} E_{o}^{\prime}-\frac{3}{2}\left(\rho_{o} \alpha\right)=0 \\
& { }^{1} \operatorname{Tanh}( \pm \delta 1= \pm 0.999987712 \simeq \pm 1
\end{aligned}
$$


Dados $\rho_{0}$ e $\alpha$ os valores interessantes de $E_{o}$ e $E_{o}^{\prime}$ são a raiz real de (3.16) e de (3.17) respectivamente. Como $\rho_{\mathrm{o}}(4+\alpha)>\rho_{\mathrm{O}} \alpha$, obviamente $\mathrm{E}_{\mathrm{o}}<\mathrm{E}_{\mathrm{o}}^{\prime}$.

As condições de contorno ainda não estão completamente definidas pois resta dar $E_{y}(x, 0)$ no intervalo $-(6 / n) \leq x \leq(6 / n)$. Em principio parece intuitivo que uma boa aproximação de $E_{y}(x, 0)$ deve evoluir de $E_{0}$ a $E_{0}^{\prime}$ segundo uma tangente hiperbólica também. Consideremos, pois, que

$$
E_{y}(x, 0)=E_{o}\left\{1+\left(\frac{E_{o}^{\prime}-E_{o}}{2 E_{o}}\right)(1+\tanh [m(x-d)]\}\right\}
$$

Se $m(x-d) \leq-6, \quad E_{y}(x, 0) \simeq E_{0} ; \quad$ também se $m(x-d) \geq 6, \quad E_{y}(x, 0) \simeq E_{o}^{\prime}$. As constantes m e d serão escolhidas de modo a forçar que a equipotencial $\Phi=0$ agora aproxime o plano $y=1$ em todo o dominio. Este é, aliás, o principal compromisso da condição de contorno em (3.18). E se tal não ocorrer, uma outra expressão para $E_{y}(x, 0)$ deve ser testada ${ }^{2}$.

\section{4 - Cálculo de Equipotenciais}

Para calcular algumas equipotenciais associadas ao sistema de dois eletródios planos, nas condiçōes descritas na seção anterior, é mais adequado reescrevermos as expansões como segue,

$$
\begin{aligned}
& E_{x}\left(x_{0}, y\right)=E_{x}\left(x_{0}, y_{0}\right)+a_{2} \Delta y+a_{5} \Delta y^{2} \\
& E_{y}\left(x_{0}, y\right)=E_{y}\left(x_{0}, y_{0}\right)+b_{2} \Delta y+b_{5} \Delta y^{2}, \\
& \rho\left(x_{0}, y\right)=\rho\left(x_{0}, y_{0}\right)+c_{2} \Delta y+c_{5} \Delta y^{2},
\end{aligned}
$$

de modo que as grandezas nelas presentes são calculadas nos pontos $\left(x_{0}, y\right)$.

A discretização do domínio está representada na matriz de pontos da Fig. - 3.3. Nela cada ponto nodal é localizado pela dupla de inteiros ( $i, j)$, onde $i$ varia ao longo de linhas horizontais espaçadas por $\Delta y$ e $j$ ao longo de verticais separadas por $\Delta \mathrm{x}$.

${ }^{2}$ Não nos ocorre uma regra para estabelecer a condição de contorno $E_{y}(x, 0)$ na situaçāo mais goral om que $p(x, 0)$ : dade por uma funçāo mais complexa. 
As grandezas $E_{x}, E_{y}$ e $\rho$ nas eqs. $(3.19 a, b, c)$ são calculadas no ponto $(i, j)$ enquanto os desenvolvimentos ocorrem em torno do ponto $(i, j-1)$ de modo que

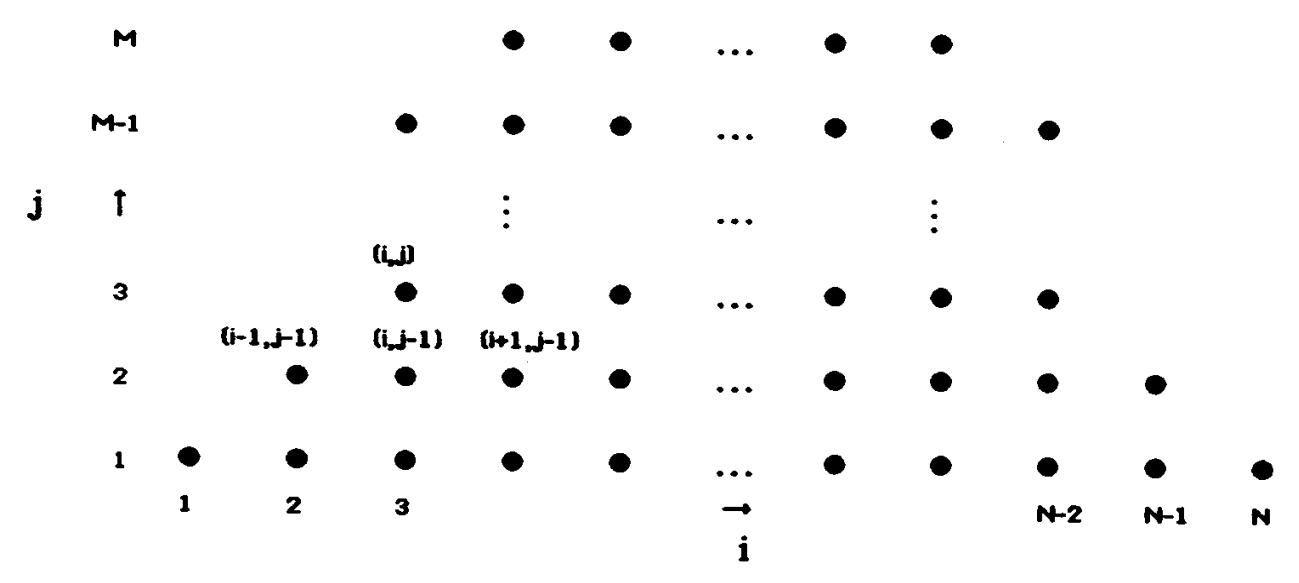

Fig. - 3.3 Discretização do espaço inter-eletródios. Os pontos $(i, 0)$ pertencem ao emissor enquanto $(i, M)$ estäo sobre o coletor.

$$
\begin{gathered}
E_{\mathbf{x}}(i, j)=E_{\mathbf{x}}(i, j-1)+a_{2}(i, j-1) \Delta y+a_{5}(i, j-1) \Delta y^{2} \\
E_{y}(i, j)-E_{y}(i, j-1)+b_{2}(i, j-1) \Delta y+b_{5}(i, j-1) \Delta y^{2} ; \\
\rho(i, j)=\rho(i, j-1)+c_{2}(i, j-1) \Delta y+c_{5}(i, j-1) \Delta y^{2} ;
\end{gathered}
$$

$$
a_{2}(i, j-1)=\left[E_{y}(i+1, j-1)-E_{y}(i-1, j-1)\right] /(2 \Delta x),
$$$$
a_{5}(i, j-1)=\frac{1}{4 \Delta x}[\rho(i+1, j-1)-\rho(i-1, j-1)]-
$$$$
-\frac{1}{2 \Delta x^{2}}[\rho(i+1, j-1)-2 \rho(i, j-1)+\rho(i-1, j-1)] ;
$$

$$
\begin{aligned}
& b_{2}(i, j-1)=\rho(i, j-1)-\left[E_{x}(i+1, j-1)-E_{x}(i-1, j-1)\right] /(2 \Delta x), \\
& b_{5}(i, j-1)=-\frac{1}{2 \Delta x^{2}}\left[E_{y}(i+1, j-1)-2 E_{y}(i, j-1)+E_{y}(i-1, j-1)\right]+\frac{1}{2} c_{2}(i, j-1) ; \\
& c_{2}(i, j-1)=-\frac{1}{E_{y}(i, j-1)}\left\{\rho^{2}(i, j-1)+\frac{E_{x}(i, j-1)}{2 \Delta x}[\rho(i+1, j-1)-\rho(i-1, j-1)]\right\}, \\
& c_{5}(i, j-1)=\frac{1}{2 E_{y}(i, j-1)}\left\{\left[\frac{E_{x}(i+1, j-1)-E_{x}(i-1, j-1)}{2 \Delta x}\right]-3 \rho(i, j-1)\right\} c_{2}(i, j-1)- \\
&-\frac{1}{4 \Delta x E_{y}(i, j-1)}[\rho(i+1, j-1)-\rho(i-1, j-1)] a_{2}(i, j-1)-\frac{E_{x}(i, j-1)}{2 E_{y}(i, j-1)} c_{4}(i, j-1) ;
\end{aligned}
$$




$$
\begin{gathered}
c_{4}(i, j-1)=-\frac{\rho(i, j-1)}{\Delta x E_{y}(i, j-1)}[\rho(i+1, j-1)-\rho(i-1, j-1)]-\frac{a_{2}(i, j-1) c_{2}(i, j-1)}{E_{y}(i, j-1)}- \\
-\frac{1}{4 \Delta x^{2} E_{y}(i, j-1)}\left[E_{x}(i+1, j-1)-E_{x}(i-1, j-1)\right][\rho(i+1, j-1)-\rho(i-1, j-1)]- \\
-\frac{E_{x}(i, j-1)}{\Delta x^{2} E_{y}(i, j-1)}[\rho(i+1, j-1)-2 \rho(i, j-1)+\rho(i-1, j-1)] .
\end{gathered}
$$

Os coeficientes estão todos representados por diferenças-finitas de $2^{\mathrm{a}}$ ordem, numa forma facilmente comparável com as suas expressões originais nas eqs. (3.6) a (3.11). Para assegurar a precisão de $2^{\text {a }}$ ordem, porém, abandona-se um ponto nos extremos de cada linha horizontal em relação à linha imediatamente inferior. Desta forma, como se vê na Fig. - 3.3, cada ponto de desenvolvimento (i,j-1) estará sempre ladeado pelos nodos $(i-1, j-1)$ e $(i+1, j-1)$, para todo ponto de cálculo $(i, j)$, permitindo assim o emprego de diferenças-centrais. Por outro lado isto resulta em um afunilamento do dominio discretizado em direção ao eletródio coletor. Entretanto se se deseja cobrir uma maior extensão desse eletródio, deve-se partir então com um número maior de pontos sobre o emissor ${ }^{3}$.

Durante o cálculo de $E_{\mathbf{x}}(i, j), E_{\mathbf{y}}(\mathrm{i}, \mathrm{j})$ e $\rho(\mathrm{i}, \mathrm{j})$, eqs. $(3.20 a, b, c)$, os pontos nodais são varridos sistematicamente da esquerda para a direita, a iniciar pela linha horizontal $(i, 2), i=2,3,4, \ldots, N-1$, e verticalmente de baixo para cima.

O potencial elétrico no ponto $(x, y)$ é por definição

$$
\Phi(x, y)=\Phi(x, 0)-\int_{0}^{y} E_{y}\left(x, y^{\prime}\right) d y^{\prime}
$$

Considerando que a componente $E_{y}(x, y)$ está ultimamente definida por um conjunto discreto de valores, optamos por aproximar a integral de (3.22a) pela regra do trapézio. Tomando explicitamente $\Phi(x, 0)=1$, o potencial elétrico em cada ponto nodal $(i, j)$ é obtido pelo seguinte algoritmo:

$$
\Phi(i, j)=1-\sum_{p=2}^{j} \frac{1}{2}\left[E_{y}(i, p-1)+E_{y}(i, p)\right] \Delta y,
$$

em que $i=M, M+1, \ldots, N-M, N+1-M$ e $j=2,3, \ldots, M$.

\footnotetext{
sobre a linha (coletor) (i,M).
} 
Finalmente, para cada $x(i)$, calculamos por interpolação linear a ordenada $Y(j)$ da equipotencial $\Phi(x(i), Y(j))=\Phi_{O}$, isto é

$$
Y(j)=y(j)-\left[\Phi_{O}-\Phi(i, j)\right] \frac{[y(j)-y(j-1)]}{[\Phi(i, j-1)-\Phi(i, j)]} .
$$

Para melhor compreensão do procedimento representado em (3.23), é preciso observar que não há necessariamente relação entre as coordenadas $x(i)$ e $y(j)$ do ponto nodal $(i, j)$ com as coordenadas do ponto de equipotencial $\Phi_{O}$, a exceção naturalmente de pontos de $\Phi_{0}-1$ e $\Phi_{0}=0$. Para simplificar os cálculos, o mais direto é arbitrar $x(i)$ como abscissa comum e sobre a linha de nodos vertical $x(i)$ determinar os valores discretos de potencial mais próximos de $\Phi_{0}: \Phi(i, j-1)$ no ponto $(i, j-1)$, de coordenadas $x(i)$ e $y(j-1)$, e $\Phi(i, j)$ no ponto $(x(i), y(j))$. A ordenada $Y(j)$ do ponto de potencial $\Phi_{0}$ é então obtida entre $y(j-1)$ e $y(j)$ por interpolação linear, conforme explicitado na eq. (3.23).

Uma outra observação deve ser feita a cerca do limite superior de $j$ em (3.22b). $M$ deve ser acrescido de algumas unidades (ver programa FORTRAN "Taylor" no Apêndice B para detalhes) de modo a permitir que $Y(j)$ na eq. (3.23) possa assumir valores superiores a 1 e assim revelar por inteiro a amplitude das flutuações da equipotencial $\Phi_{0}=0$ em torno do plano $y=1$. Em última análise a escolha dos melhores valores de $m$ e d da condição de contorno $E_{y}(x, 0)$, eq. (3.18), estará determinada pela menor dessas flutuações.

\section{5 - Resultados e Discussão}

Os nossos resultados estão apresentados em grupos de três gráficos rotulados (os gráficos) invariavelmente pelas letras $\underline{A}, \underline{B}$ e $\underline{C}$. Cada grupo, a que nos referimos por "Fig.-3.4", "Fig.-3.5", etc, reúne uma amostra de equipotenciais calculadas para um caso particular (uma escolha dos parâmetros $\rho_{O}$, n e $\alpha$ ) da densidade de carga injetada, eq. (3.14). Nos gráficos $\underline{A}$ nós mantemos constante um dos dois parâmetros do campo de injeção (eq. (3.18)), d-0.0, e variamos o outro, m, para obter a equipotencial $\Phi_{0}=0$ mais próxima do plano $y=1$. 
Esta equipotencial está marcada com círculos para ser destacada das demais. Nos gráficos $\underline{B}$ construidos em escala idêntica à dos gráficos $\underline{A}$ (1.00V:10.04in, para as ordenadas), repetimos o mesmo tipo de experimentação numérica para selecionar 0 valor adequado de d, tendo-se mantido $m$ no valor previamente selecionado. $\dot{E}$ oportuno observar que insistindo com a experimentação numérica não conseguimos refinar estes valores. Por último, nos gráficos $\underline{C}$ (1V:2.51in, para as ordenadas), temos a amostra de equipotenciais propriamente dita, cujo cálculo é processado tendo em conta os "melhores" m ed.

A eficiência da solução formulada neste capitulo depende fundamentalmente do grau de acerto no qual é construida a condição de contorno no campo elétrico sobre o emissor e, em segundo plano, da ordem do truncamento das expansões (3.2) a (3.4).

O número de termos a somar nas expansões á ditado pelos valores de $\rho_{o}$, isto é, pela magnitude da carga injetada. Considerada a segunda ordem, a variação de $\rho_{0}$ através dos valores $0.5,1.0$ e 2.0 , como se pode observar da Fig.-3.4 à Fig.-3.10, muda consideravelmente $\circ$ padrão de equipotenciais do campo, de tal modo que, no último destes casos (Fig.-3.10), a equipotencial $\Phi_{0}=0.0$ já não pode representar nem mesmo grosseiramente um eletródio plano. Entretanto termos de segunda ordem são o bastante para tratar com os valores de $\rho_{0}$ entre 0.0 e 1.0, pois obtem-se equipotenciais de coletor suficientemente planas e os resultados estão em muito boa concordância com os dados que calculamos com base nas ideias do método hodográfico de Budd \& Wheeler [4]. Uma comparação está feita na Fig. $-3.8(\mathrm{C})$ onde representamos os dados de referência pelos pequenos circulos.

Está subentendido que enquanto se observa a dependência da solução com os valores de $\rho_{O}$, os demais parâmetros da carga injetada (n e $\alpha$ ) são mantidos nos mesmos niveis constantes. Podemos observar por todos os resultados que a solução é também sensivel a variações, mesmo pequenas, desses parâmetros. Assim, n, que tem $\circ$ papel de estreitar ou alargar a extensão de eletródio em que a densidade de carga injetada varia marcadamente, tem relação idêntica com as equipotenciais. Os pequenos valores de $n$ seriam incovenientes porque o cálculo 
deveria cobrir uma maior extensão do eletródio emissor de modo a permitir uso mais correto dos valores assintóticos $\rho_{O}(4+\alpha)$ e $\rho_{O} \alpha$ da carga injetada. Já os grandes valores tornam muito abrupta a variação de $\rho(x, 0)$ o que exige um refinamento crescente na discretização do domínio, sobretudo na direção paralela aos eletródios. Na prática, por uma razão que ainda não compreendemos, este refinamento é limitado. O máximo que conseguimos é $\Delta x-30 \Delta y$ e em todos os nossos cálculos tomamos $\Delta y=0.005$. O parāmetro a mantém com as equipotenciais relação parecida com a existente entre elas e $\rho_{O} .0$ crescimento de ambos, $\alpha$ e $\rho_{O}$, faz crescer a carga injetada e o efeito disto é um deslocamento das equipotenciais em direção ao coletor. Porém, como a variação relativa que $\alpha$ produz em $\rho(x, 0)$ é bem maior na região $x>0$, é á também onde ocorrem, com $\alpha$, as mudanças mais acentuadas nas equipotenciais.

$O$ ajuste da equipotencial $\Phi_{0}-0.0$ com 0 plano $y=1.0$ conduz a relações de dependência muito interessantes entre campo de injeção e a carga injetada. Nota-se que os "melhores" valores de m são quase sempre iguais aos de n; para $\rho_{0}$ e $\alpha$ fixos, o produto nxd é uma constante; por último, mantendo-se $n$ e $\alpha, 0$ crescimento geométrico de $\rho_{0}$ por um fator $2\left(\rho_{0}=0.5,1.0,2.0\right) \mathrm{faz} \mathrm{d}$ crescer aritmeticamente com razão $0.4(d=0.6,1.0,1.4)$. 

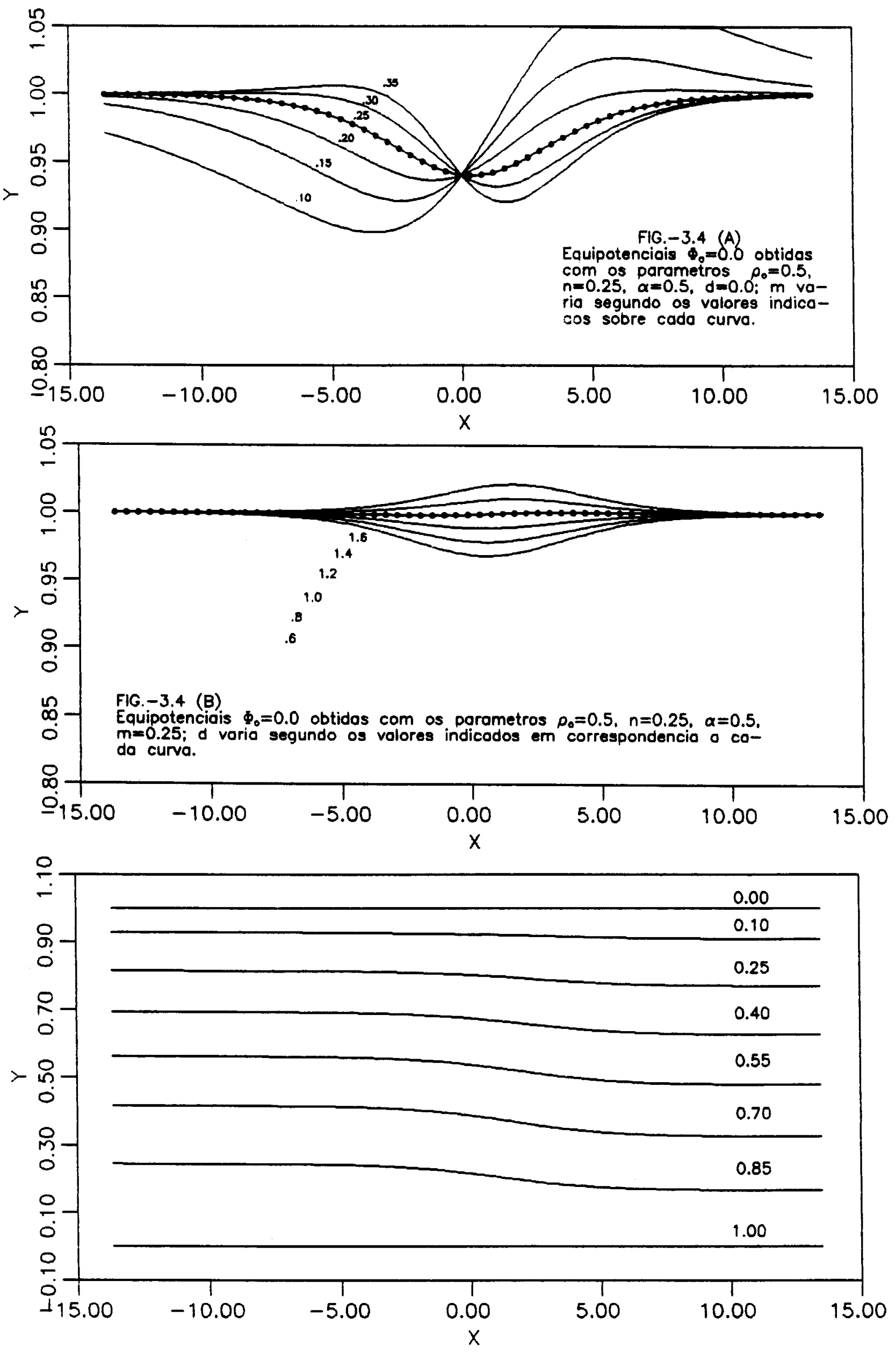

FIG. -3.4 (C)

Equipotenciais calculadas com os parametros $\rho_{0}=0.5, n=0.25, \alpha=0.5$; $m=0.25, d=1.2$. Os valores de $\Phi_{0}$ estao indicados sobre cada curva. 

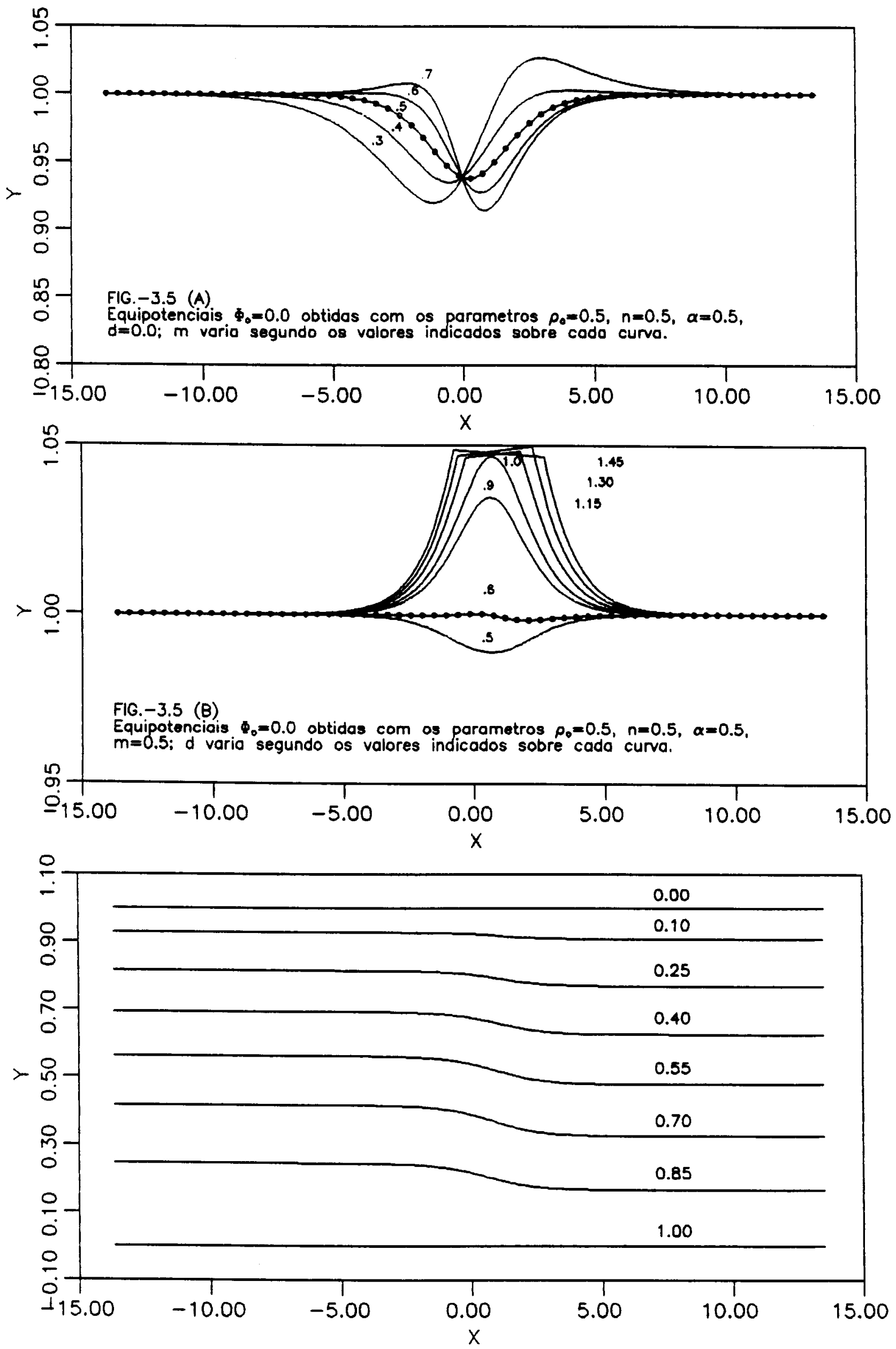

FIG. -3.5 (C)

Equipotenciais calculadas com os parametros $\rho_{0}=0.5, n=0.5, \alpha=0.5$; $m=0.5, d=0.6$. Os valores de $\Phi_{0}$ estao indicados sobre cada curva. 

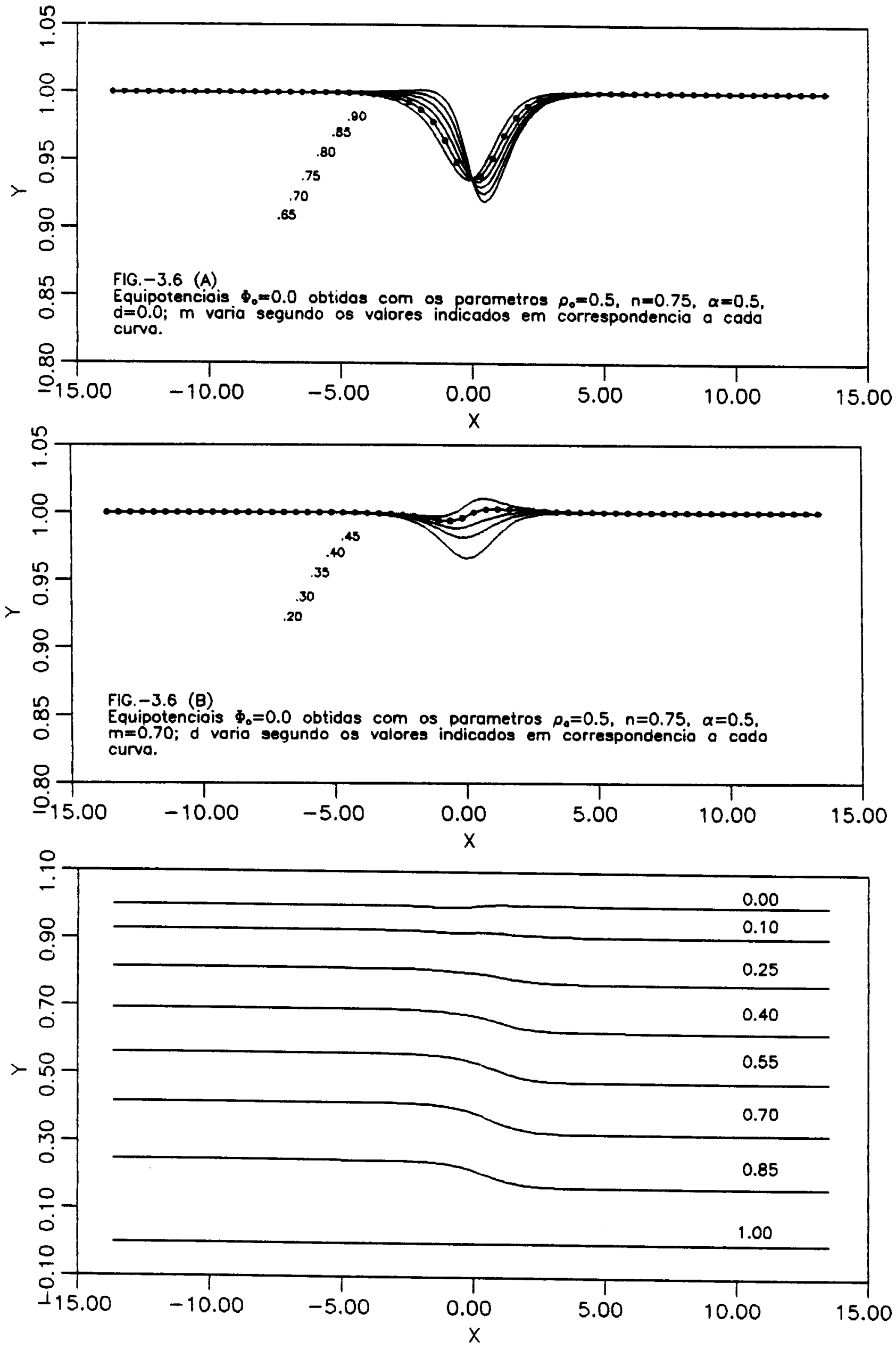

FIG. -3.6 (C)

Equipotenciais calculados com os parametros $\rho_{0}=0.5, n=0.75, \alpha=0.5$; $m=0.70, d=0.40$. Os valores de $\Phi_{0}$ estao indicados sobre cada curva. 

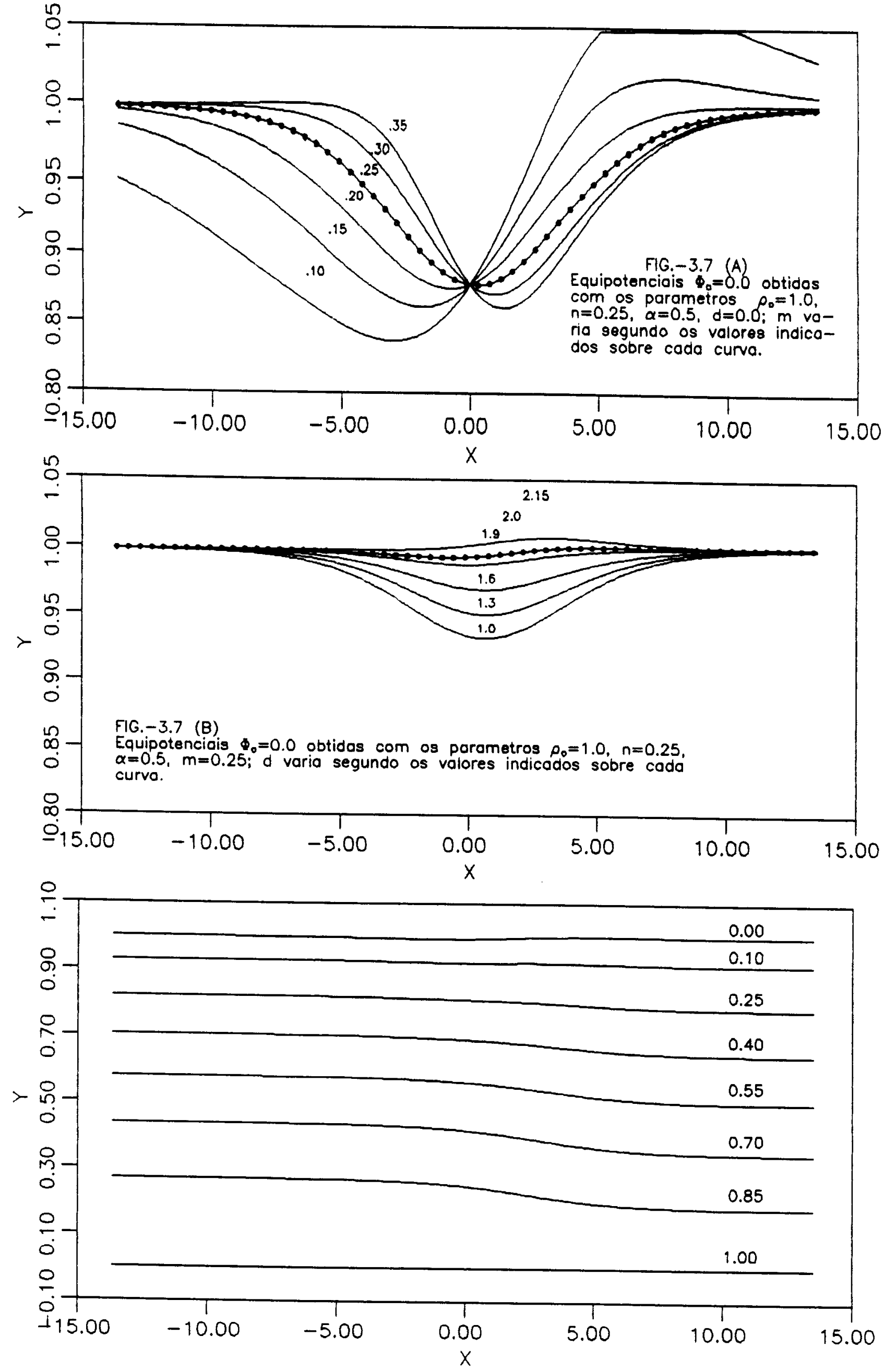

FIG. -3.7 (C)

Equipotenciais calculadas com os parametros $\rho_{0}=1.0, n=0.25, \alpha=0.5$; $\mathrm{m}=0.25, d=2.0$. Os valores de $\Phi_{0}$ estao indicados sobre cada curva. 

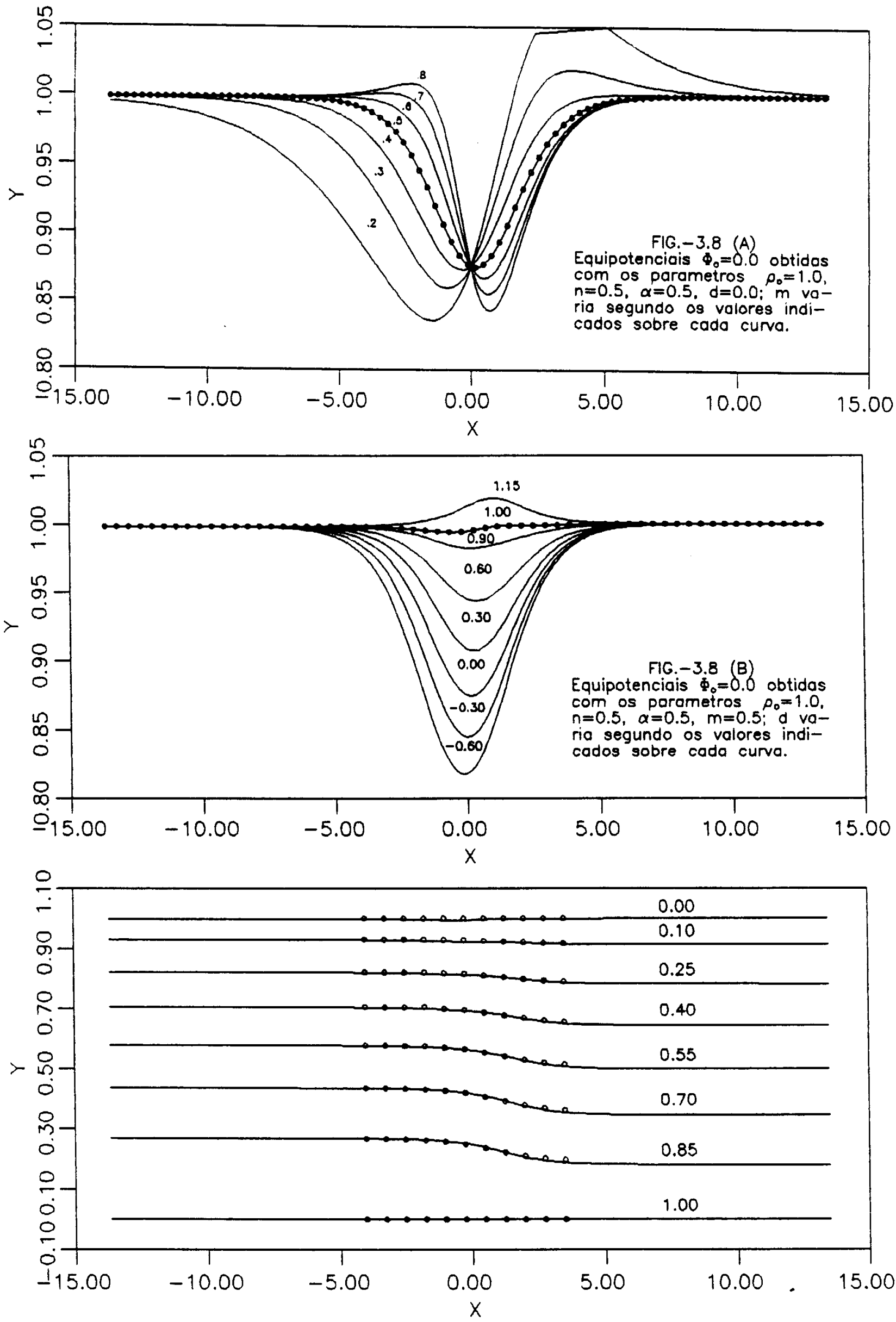

FIG. -3.8 (C)

(-) Equipotenciais obtidas com os parometros $\rho_{0}=1.0, n=0.5, a=0.5$; $m=0.5, d=1.00$. Os valores de $\Phi_{0}$ estao indicados sobre cada curva. (o) Equipotenciais calculadas pelo metodo hodografico (Cap.V) nas mesmas condicoes de carga espacial injetoda. 

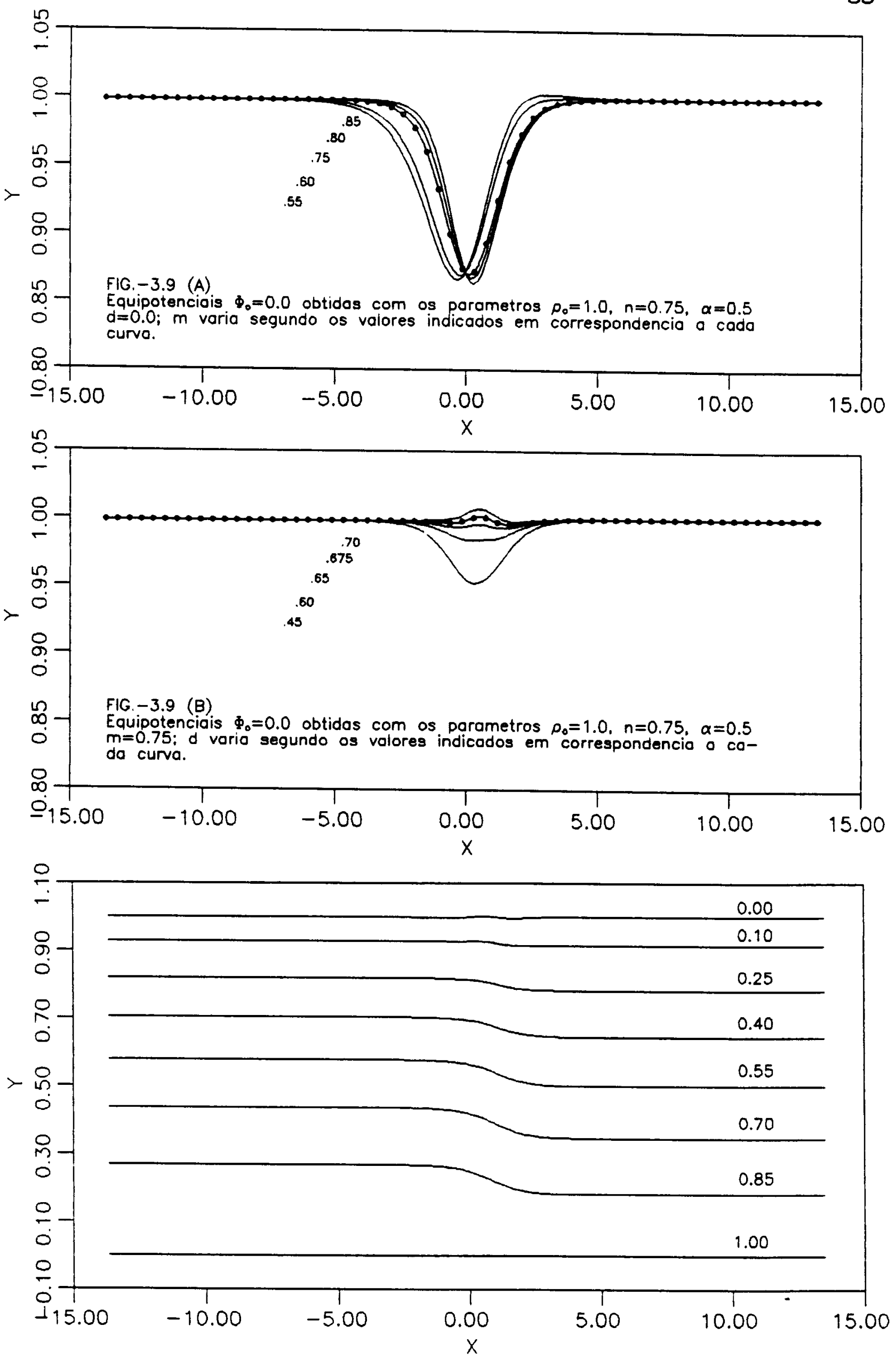

FIG. -3.9 (C)

Equipotenciais calculadas com os parametros $\rho_{0}=1.0, n=0.75, \quad \alpha=0.5$; $\mathrm{m}=0.75, d=0.675$. Os valores de $\Phi_{0}$ estao indicados sobre cada curva. 

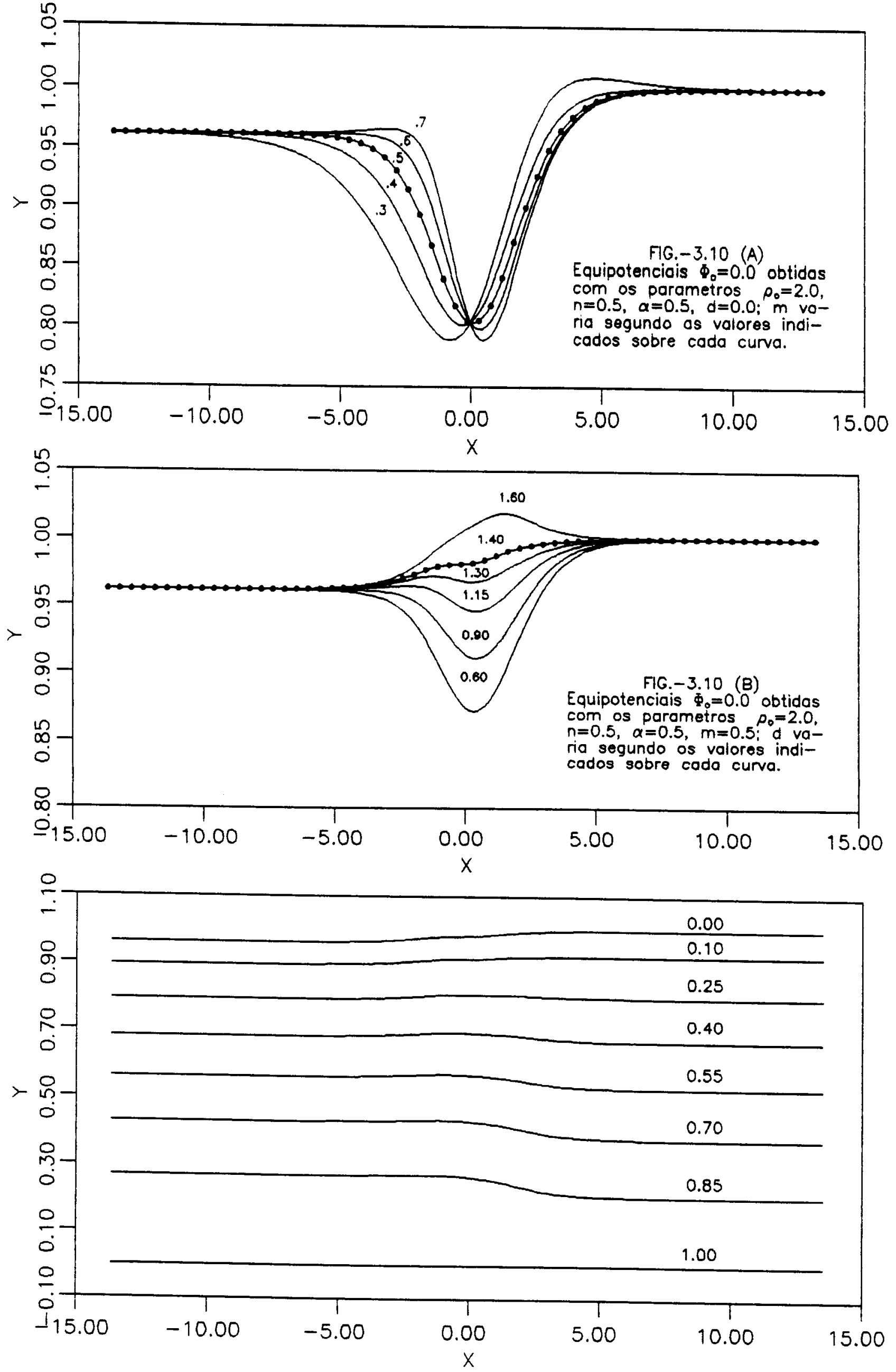

FIG. -3.10 (C)

Equipotenciais calculadas com os parametros $\rho_{0}=2.0, n=0.5, \alpha=0.5$; $\mathrm{m}=0.5, d=1.4$. Os valores de $\Phi_{0}$ estao indicados sobre cada curva. 

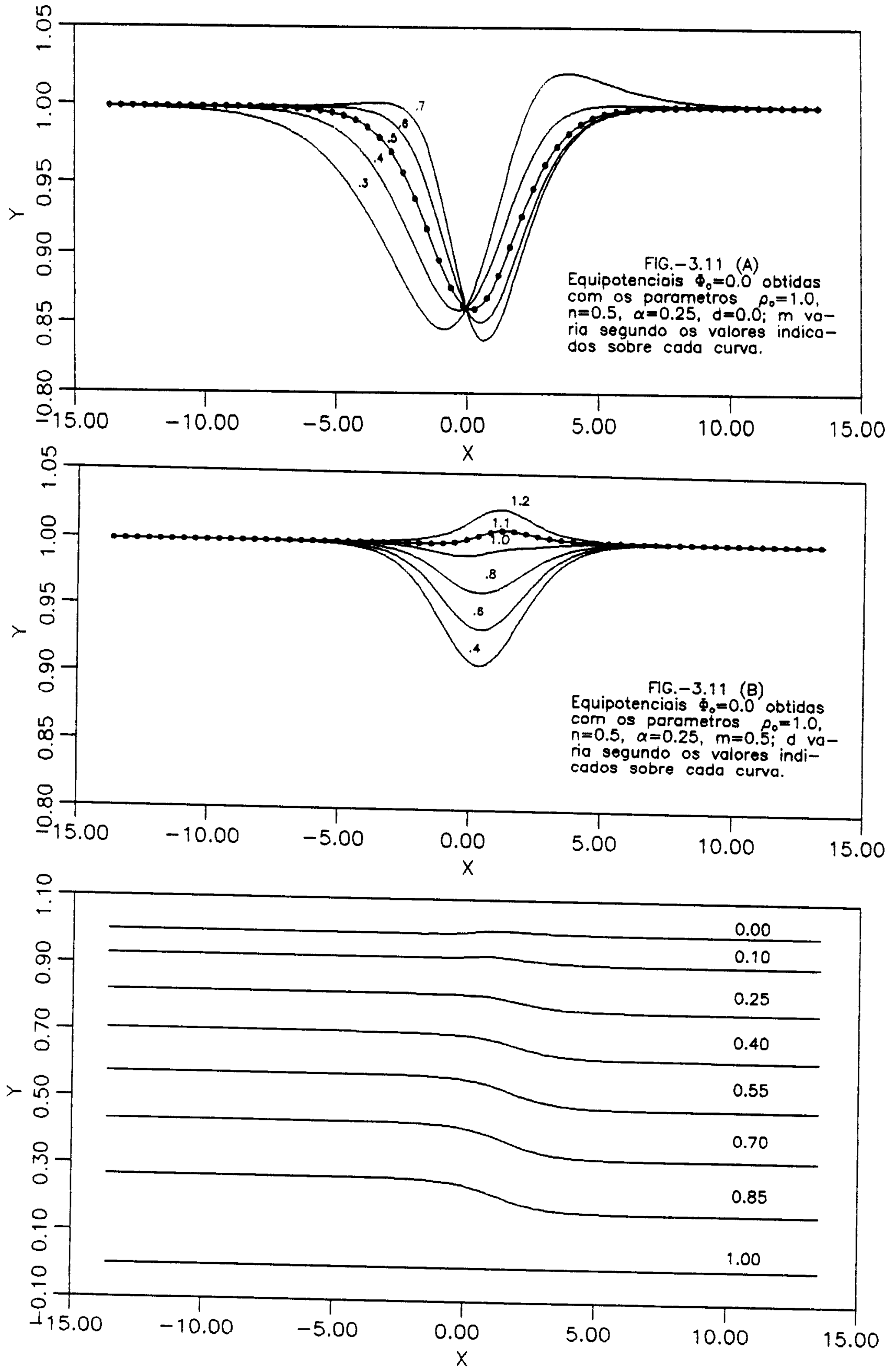

FIG. -3.11 (C)

Equipotenciais calculados com os parametros $\rho_{0}=1.0, n=0.5, \alpha=0.25$;

$\mathrm{m}=0.5, d=1.1$. Os valores de $\Phi_{0}$ estao indicados sobre cada curva. 

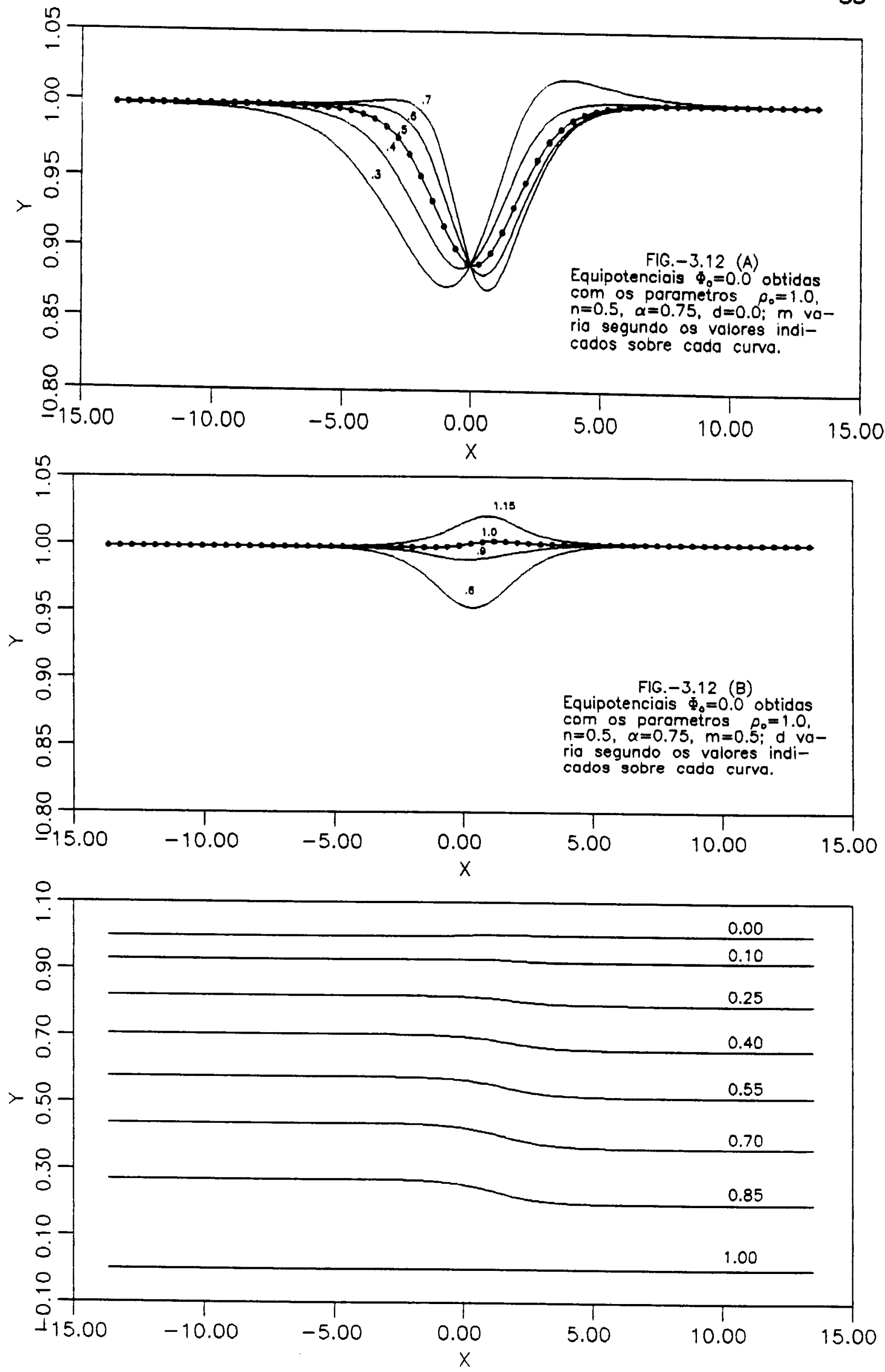

FIG. -3.12 (C)

Equipotenciais calculadas com os parametros $p_{0}=1.0, n=0.5, \alpha=0.75$; $m=0.5, d=1.0$. Os valores de $\Phi_{0}$ estao indicados sobre cada curva. 


\section{APITULO IV}

\section{O Método de Budd \& Wheeler e as Equaçōes de Carga Espacial}

\section{1 - Introdução}

O método de Budd \& Wheeler [4] para o tratamento das equaçōes de carga espacial envolve aspectos analíticos e numéricos. Quanto aos primeiros, o método reedita as equaçōes em formas mais adequadas a aproximaçōes numéricas tanto quanto precisas usando técnicas de diferenças finitas. Enquanto numérico, o método discute e emprega algoritmos iterativos para resolver o problema algébrico resultante.

A análise está fundamentada na reformulação das equaçōes de carga espacial em termos de um sistema de coordenadas ortogonais planas, cujas curvas coordenadas são as equipotenciais e as linhas do campo da própria carga espacial. Especificamente, faz-se uma transformação ${ }^{1}$ do sistema ortogonal de coordenadas espaciais, $(x, y)$, ao sistema $(\Phi, \psi)$ em que $\Phi$ é o potencial elétrico da carga espacial e $\psi$ é uma função cujas curvas de nivel $(\psi=$ cte.) são definidas como as linhas de campo associadas.

Sob uma tal transformação (a) as equações de carga espacial passam a exprimir $x, y, 1 / \rho$ e outras grandezas de campo como funções de $\Phi$ e $\psi-$ e é de fato nesta perspectiva que elas são resolvidas; (b) os dominios de solução, independentemente da natureza geométrica de suas fronteiras, são sempre transformados em um retângulo no espaço $(\Phi, \psi)$. É necessário, porém, que essas fronteiras possam ser originalmente construidas por segmentos de equipotenciais e de linhas de campo, em principio conhecidas; (c) as curvas caracteristicas do sistema das equações de carga espacial tornam-se coincidentes com as curvas coordenadas $\psi$-cte., consequência da construção do sistema $(\Phi, \psi)$. Isto tem a

\footnotetext{
'For falta de um termo mais aceitável, os autores denominaram esta transformaçāo de "hodográfica" e por extensāo também o seu método. Nós preforimos chamá-1o de "Método de Budd \& Wheeler".
} 
vantagem de tornar mais fácil a integração da componente hiperbólica do sistema, eq. (2.11a) ou, mais tarde, eq. (4.11b).

Do ponto de vista numérico a transformação $(x, y) \rightarrow(\Phi, \psi)$ equivale à geração de uma grade ortogonal de corpo ajustado. Neste domínio computacional linhas nodais são dispostas paralelas às curvas coordenadas $\Phi=c t e$ de tal modo que as fronteiras do dominio passam obrigatoriamente por pontos nodais, não existindo necessidade de interpolações, quando da aplicação das condições de contorno. O uso de uma grade ortogonal de corpo ajustado é assim muito importante na resolução numérica de sistemas diferenciais parciais em que as condiçōes de contorno têm influência dominante no caráter das soluções.

\section{2 - Condição de Ortogonalidade do Sistema $(\Phi, \Psi)$}

Em geral uma base de vetores unitários $\left\{\hat{e}_{1}, \hat{e}_{2}\right\}$ associada a um sistema de coordenadas $(\Phi, \psi) \dot{e}$ definida como

$$
\begin{aligned}
& \hat{\mathbf{e}}_{1}=\frac{1}{h_{1}}\left(x_{*} \hat{i}+y_{4} \hat{j}\right), \\
& \hat{\mathbf{e}}_{2}=\frac{1}{h_{2}}\left(x_{\phi} \hat{i}+y_{\infty} \hat{j}\right),
\end{aligned}
$$

em que $h_{1}$ e $h_{2}$, os fatores de escala, são dados por

$$
\begin{aligned}
& h_{1}=\left(x^{2}+y_{\psi}^{2}\right)^{1 / 2} \\
& h_{2}=\left(x_{p}^{2}+y_{p}^{2}\right)^{1 / 2}
\end{aligned}
$$

O sistema $(\Phi, \psi)$ é dito ortogonal quando em cada ponto $(\Phi, \psi) \quad \hat{e}_{1} \cdot \hat{e}_{2}=0$ consequentemente as primeiras derivadas parciais de $\mathrm{x}$ e $\mathrm{y} \operatorname{com}$ respeito a $\Phi$ e $\psi$ devem satisfazer à relação de ortogonalidade

$$
x_{\phi} x_{\psi}+y_{4} y_{y}=0
$$

Se $d \Phi$ e $d \psi$ são variações infinitesimais em $\Phi$ e $\psi$, então 


$$
\begin{aligned}
& d \Phi=\Phi_{x} d x+\Phi_{y} d y \\
& d \psi=\psi_{x} d x+\psi_{y} d y
\end{aligned}
$$

Expressando os infinitésimos $\mathrm{dx}$ e dy em termos de $\Phi$ e $\psi$ e substituindo-os em (4.4a), encontram-se

$$
\begin{aligned}
& \mathrm{d} \Phi=\left(\Phi_{\mathrm{x}} \mathrm{x}_{\phi}+\Phi_{\mathrm{y}} \mathrm{y}_{\phi}\right) \mathrm{d} \Phi+\left(\Phi_{\mathrm{X}} \mathrm{x}_{\psi}+\Phi_{\mathrm{y}} \mathrm{y}_{\phi}\right) \mathrm{d} \psi, \\
& \mathrm{d} \psi=\left(\psi_{\mathrm{x}} \mathrm{x}_{\phi}+\psi_{\mathrm{y}} \mathrm{y}_{\phi}\right) \mathrm{d} \Phi+\left(\psi_{\mathrm{x}} \mathrm{x}_{\phi}+\psi_{\mathrm{y}} \mathrm{y}_{\phi}\right) \mathrm{d} \psi .
\end{aligned}
$$

Igualando os coeficientes de $\mathrm{d} \Phi$ e de $\mathrm{d} \psi$ entre os lados das duas últimas igualdades, resultam

$$
\begin{gathered}
{\left[\begin{array}{l}
1 \\
0
\end{array}\right]=\left[\begin{array}{ll}
\Phi_{\mathrm{x}} & \Phi_{\mathrm{y}} \\
\psi_{\mathrm{x}} & \psi_{\mathrm{y}}
\end{array}\right]\left[\begin{array}{l}
\mathrm{x}_{*} \\
\mathrm{y}_{*}
\end{array}\right],} \\
{\left[\begin{array}{l}
0 \\
1
\end{array}\right]=\left[\begin{array}{ll}
\Phi_{\mathrm{x}} & \Phi_{\mathrm{y}} \\
\psi_{\mathrm{x}} & \psi_{\mathrm{y}}
\end{array}\right]\left[\begin{array}{l}
\mathrm{x}_{\boldsymbol{\psi}} \\
\mathrm{y}_{\boldsymbol{\psi}}
\end{array}\right] .}
\end{gathered}
$$

A solução do sistema (4.5) para $\Phi_{\mathrm{x}}, \Phi_{\mathrm{y}}, \psi_{\mathrm{x}}$ e $\psi_{\mathrm{y}}$ é

$$
\Phi_{\mathbf{X}}=\mathbf{J} \mathrm{y}_{\emptyset}, \quad \Phi_{\mathrm{y}}=-\mathbf{J} \mathbf{x}_{\phi}, \quad \psi_{\mathrm{x}}=-\mathbf{J} \mathrm{y}_{\phi}, \quad \psi_{\mathrm{y}}=\mathbf{J} \mathrm{x}_{\phi},
$$

em que I é o jacobiano da transformação de coordenadas, isto é,

$$
\mathbb{J}=\Phi_{\mathbf{x}} \psi_{\mathbf{y}}-\Phi_{\mathbf{y}} \psi_{\mathbf{x}}
$$

Os resultados (4.6) permitem reescrever a condição de ortogonalidade (4.3) na forma

$$
\boldsymbol{\nabla} \boldsymbol{\Phi} \cdot \boldsymbol{\nabla} \psi=0
$$

a qual reafirma que $\circ$ vetor campo elétrico é tangente às curvas $\psi$-cte. 


\section{3 - Transformação das Equações sob a Mudança de Coordenadas $(x, y) \rightarrow(\Phi, \psi)$}

A condição (4.8) é geralmente satisfeita quando

$$
\Phi_{x}=\lambda \psi_{y}, \quad \Phi_{y}=-\lambda \psi_{x},
$$

em que $\lambda$ é uma função a ser determinada. Isto quer dizer que para definir-se o sistema de coordenadas $(\Phi, \psi)$ faz-se necessário mais uma variável, $\lambda$.

As equações de carga espacial podem agora ser reescritas na forma de um conjunto de equações diferenciais de primeira ordem, a saber,

$$
\begin{gathered}
-\rho=\lambda_{\mathbf{X}} \varpi_{\mathrm{Y}}-\lambda_{\mathrm{Y}} \varpi_{\mathrm{X}}, \\
\rho^{2}=\rho_{\mathrm{X}} \Phi_{\mathrm{X}}+\rho_{\mathrm{Y}} \Phi_{\mathrm{Y}}, \\
\Phi_{\mathrm{X}}=\lambda \psi_{\mathrm{Y}}, \quad \Phi_{\mathrm{Y}}=-\lambda \psi_{\mathrm{X}} .
\end{gathered}
$$

A primeira destas é deduzida da equação de Poisson $\left(\nabla^{2} \Phi=-\rho\right)$ em combinação com as identidades (4.9) e a segunda é uma outra versão da equação característica Substituindo em (4.10a) as derivadas $\lambda_{X}, \lambda_{y}, \rho_{X}$ e $\rho_{y}$ por suas expressões em $\Phi$ e e eliminando do resultado todas as derivadas de $\Phi$ e $\psi$ por intermédio das eqs. (4.6) a (4.8), o sistema pode ser simplificado a

$$
\begin{gathered}
\lambda_{\phi}=-\lambda \rho\left(x_{\Phi}^{2}+y_{\phi}^{2}\right), \\
(1 / \rho)_{\phi}=-\left(x_{\phi}^{2}+y_{\phi}^{2}\right), \\
y_{\phi}=\lambda x_{\phi} \quad x_{\phi}=-\lambda y_{\phi} .
\end{gathered}
$$

As duas primeiras equaçōes podem ser resolvidas facilmente para dar $\lambda=a(\psi) \sigma$, com $\sigma=1 / \rho$ e a(屯) uma função arbitrária de $\psi$, em termos do que se deduzem finalmente 


$$
\begin{gathered}
y_{\phi}=a(\psi) \sigma x_{\phi}, \\
x_{\phi}=-a(\psi) \sigma y_{\phi} \\
\sigma_{\phi}=-\left(x_{\phi}^{2}+y_{\phi}^{2}\right) .
\end{gathered}
$$

Este conjunto de equações compreende um sistema elíptico, eq. (4.11a), acoplado a uma equação diferencial ordinária para $\sigma$ - sobre as caracteristicas $\psi=c$ cte., obviamente. Nele está subentendida a existência da transformação inversa ${ }^{2}$ de $(\Phi, \psi)$ a $(x, y)$, significando fisicamente que para cada valor do potencial, ao longo de cada linha de campo, the corresponde um único ponto no espaço.

A intensidade de campo elétrico é facilmente dedutivel a

$$
|E|=\left(x_{\psi}^{2}+y_{*}^{2}\right)^{-1 / 2}=a(\psi) \sigma\left(x_{\psi}^{2}+y_{\psi}^{2}\right)^{-1 / 2},
$$

sendo a última versão muito importante para se estabelecer a relação entre a carga emitida e o campo de emissão.

É importante observar que o sistema (4.11) também se aplica corretamente à solução do problema harmônico associado $(p-0)$, bastando para tanto usar $\sigma=1$. Para demonstrá-lo é necessário apenas partir da equação de Laplace e transformá-la no resultado $J \lambda_{\$}=0$, por aplicações seguidas das eqs. (4.9), (4.6) e regra da cadeia. A solução deixa de $\operatorname{ser} \lambda-a(\psi) \sigma$, quando era $\rho \neq 0$, para ser agora $\lambda=\lambda(\psi) \equiv a(\psi)$.

\subsection{1 - O Significado de $a(\psi)$}

Conclui-se imediatamente de (4.12) que $\circ$ produto $|a(\psi)| \sigma$ está relacionado com a natureza da transformação $(x, y) \rightarrow(\Phi, \psi)$ através da razão entre seus coeficientes métricos, ver (4.2),

$$
|\mathrm{a}(\psi)| \sigma=\frac{\mathrm{h}_{2}}{\mathrm{~h}_{1}} .
$$

${ }^{2}$ Budd \& Wheeler [4] justificam-na apelando a um principio de máximo para segundo o qual se $\left|\nabla_{\Phi}\right|$ se anula om um ponto isolado do domínio, ontäo ele não deve ser nem um máximo e nom um mínimo locai do $\$$. 
Em decorrência pode-se afirmar seguramente que $|a(b)| \sigma$ é, em parte, determinante da geometria do reticulado de campo.

Seria, porém, bem mais interessante buscar o significado de $a(\psi)$ isoladamente. Considerem-se, uma após outra, as definições da densidade de corrente, J, e do gradiente de $\Phi$ nas coordenadas $(\Phi, \downarrow)$ :

$$
\mathrm{J}=\rho \mathrm{E}=-\rho \nabla \Phi=-\frac{1}{\sigma} \frac{\hat{\mathrm{e}}_{1}}{\mathrm{~h}_{1}}=-\frac{|\mathrm{a}(\boldsymbol{\phi})|}{\mathrm{h}_{2}} \hat{\mathrm{e}}_{1}
$$

Aqui se usou (4.13) para eliminar $1 / \sigma h_{1}$ no penúltimo termo. Por outro lado, o fluxo de corrente dI através do elemento de arco $d s=h_{2} d \psi$, tomado ao longo de uma equipotencial entre $\psi$ e $\psi+d \psi$, é dado por

$$
d I=J \cdot\left(h_{2} d \psi\right)\left(-\hat{e}_{1}\right)-|a(\psi)| d \psi
$$

Portanto, apreende-se que a( $\psi$ ) representa, no novo espaço de coordenadas, a taxa de variação do fluxo de corrente por unidade de $\psi$.

\subsection{2 - Condições de Contorno Apropriadas ao Sistema de Equaçōes (4.11) em um Dominio Simplesmente Conexo}

\footnotetext{
Como mencionado no cap.l, os interesses neste trabalho estarão voltados exclusivamente para a solução de problemas de carga espacial em um sistema de dois eletródios planos paralelos e/ou, quando muito, de dois eletródios que nalguma extensão apresentam ainda essa geometria planar.
}

Em terminologia matemática, cada um desses sistemas constitui o que se define, assim como na Fig.-4.1, um dominio curvilíneo simplesmente conexo. A fronteira $\partial \Omega_{0}$ representa o eletródio emissor ou a fonte da carga espacial, enquanto $\partial \Omega_{1}$ simboliza o coletor (em geral aterrado). Estes eletródios são mantidos aos potenciais constantes $\Phi_{0}$ e $\Phi_{1}$, sendo $\Phi_{0}>\Phi_{1}$ e $\Phi_{1}=0$ sem perda de generalidade. Por conveniência, a superfície $\partial \Omega_{0}$ é descrita parametricamente por $x=l f_{1}(\theta)$ e $y=l g_{1}(\theta)$, onde $l$ é uma distância típica entre os eletródios; e as demais, 
$\partial \Omega_{\mathrm{i}}(\mathrm{i}=1,2,3)$, são definidas nas formas $\mathrm{H}(\mathrm{x} / l, \mathrm{y} / l)-0, \mathrm{G}_{2}(\mathrm{x} / l, \mathrm{y} / l)=0$ e $\mathrm{G}_{3}(\mathrm{x} / l, \mathrm{y} / l)=0$, respectivamente. Sobre os contornos laterais $\partial \Omega_{2}$ e $\partial \Omega_{3}$ impõe-se que $\partial \Phi / \partial n=0,0$ que equivale a restringi-los simplesmente a linhas de campo e/ou de corrente.

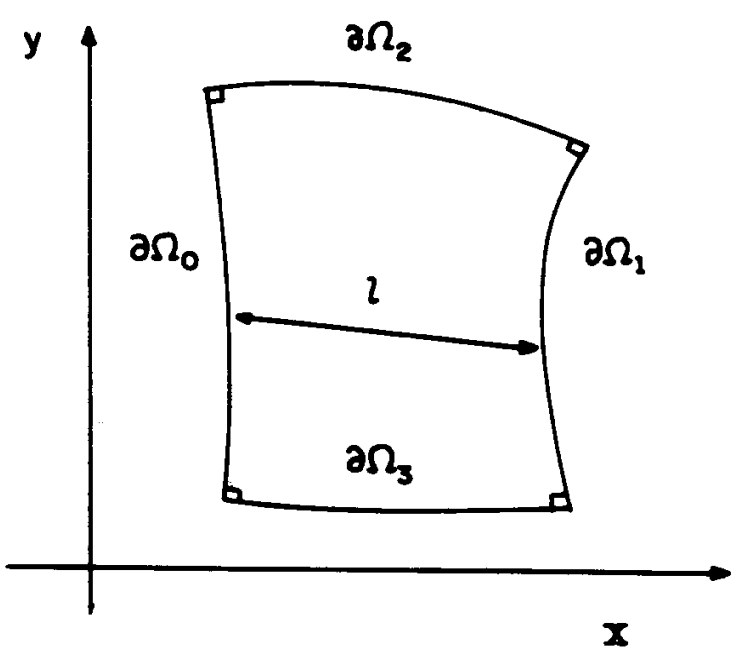

FIG.-4.1 Dominio simplesmente conexo representação dos sistemas de eletródios estudados.

Antes de estabelecer as condiçōes de contorno em $x, y, \sigma$ e $a(\phi)$ a que deverão estar sujeitas as eqs. do sistema (4.11), de modo a se ter o problema completamente definido no espaço $(\Phi, \psi)$, é necessário relacionar a variável $\downarrow$ com a geometria dos sistemas de eletródios. O procedimento é associar cada linha de campo $(\psi=$ cte.) a uma posição sobre a superficie condutora da qual emanam. Isto pode ser feito das mais diferentes formas, contanto que a correspondência seja única. Em particular, para um dominio simplesmente conexo, Fig. - 4.1, uma opção ${ }^{3}$ de Budd \& Wheeler [4] é fazer $\psi-\theta$, em que $\theta$ é o parâmetro em termos do qual se descreve a superfície emissora $\partial \Omega_{0}$. Os limites de $\theta$, ou optativamente de $\psi\left(\psi_{0} e\right.$ $\psi_{1}$ ) são definidos segundo a conveniência de cada problema e $0 \leq \Phi \leq 1$, $\psi_{0} \leq \boldsymbol{\psi} \leq \boldsymbol{\psi}_{1}$, define a região retangular em que são mapeados os domínios físicos, como já mencionado no inicio deste capítulo.

As condiçōes de contorno para as eqs. (4.11) são então, (em grandezas adimensionais),

${ }^{3}$ Os procedimentos de parametrização om domínios uniconexos a conexos múltuplos estão descritos. respectivamente, nas duas últimas secçōes de Budd \& Wheelor $[4]$ e om Budd \& whoeler $[26]$. 


$$
\begin{gathered}
F(\psi,|E|, 1 / \sigma)=0, \quad x=f_{1}(\psi), \quad y=g_{1}(\psi), \quad \psi_{0} \leq \psi \leq \psi_{1}, \quad \Phi=1 ; \\
H(x, y)=0, \quad \psi_{0} \leq \psi \leq \psi_{1}, \quad \Phi=0 ; \\
G_{2}(x, y)=0 \quad \text { em } \psi=\psi_{1} ; \\
G_{3}(x, y)=0 \quad \text { em } \psi=\psi_{0} .
\end{gathered}
$$

Observe-se que se $x$ e y estão prescritos em $\Phi=1$, o problema definido por (4.11) juntamente com as condições (4.16) parece estar super determinado. Todavia em nenhum ponto do domínio os valores de $a(\not)$ ou suas derivadas têm sido fornecidos. A liberdade em se definir $\mathrm{x}$ e $\mathrm{y}$ como funções de $\psi$ em $\Phi-1$ é controlada por a $(\psi)$ que passa a ser parte da solução do problema. Em outras palavras, para cada escolha de parametrização da superfície emissora, ter-se-á uma nova função $a(\psi)$ tal que, sempre, $I=A\left(\psi_{0}\right)-A\left(\psi_{1}\right)$, em que $A(\psi)=\int_{\psi} a\left(\psi^{\prime}\right) d \psi^{\prime}$ e $\underline{\text { é }}$ 。 fluxo total de corrente entre $\psi_{0}$ e $\psi_{1}$.

\section{4 - O Método Numérico}

Para resolver numericamente as equações de carga espacial (4.11), Budd \& Wheeler [4] empregam um esquema padrão, cuja implementação depende da conexidade do domínio original e, mesmo em se restringindo à classe representada na Fig. - 4.1, da forma particular que tomam as condições de contorno definidas em (4.16).

O esquema compreende dois passos distintos, porém executados de uma tal forma que, no conjunto, garante-se o acoplamento das componentes (eliptica e hiperbólica) do problema. São eles:

1. Dado $\sigma(\Phi, \psi)$, resolve-se o problema elíptico (4.11a) para $x(\Phi, \psi), y(\Phi, \psi)$ e $a(\psi)$.

Aqui emprega-se invariavelmente uma formulação de diferenças finitas, posto que a transformação $(x, y) \rightarrow(\Phi, \psi)$ permite sempre o uso de uma grade retangular uniforme.

2. Dados $\mathrm{x}(\Phi, \psi), \mathrm{y}(\Phi, \psi)$ e $a(\psi)$, determina-se $\sigma(\Phi, \psi)$ a partir da equação diferencial ordinária 


$$
\sigma_{\Phi}=-\left(\mathrm{x}_{\Phi}^{2}+\mathrm{y}_{\Phi}^{2}\right)
$$

O processo numérico pode ser iniciado com uma solução tentativa para $\sigma(\Phi, \psi)$ e prossegue em iteração dos passos $\underline{1}$ e $\underline{2}$ a té a convergência (se houver). 0 passo 1 é o mais dispendioso em implementação e processamento e onde também ocorrem as maiores dificuldades numéricas.

A condição inicial em $\sigma(\Phi, \psi)$, necessária à integração de (4.17a), é dada pelas relações funcionais

$$
\begin{gathered}
\mathrm{F}(\psi,|\mathrm{E}|, 1 / \sigma)=0, \quad \Phi=1, \\
\sigma \mathrm{a}(\psi)=|\mathrm{E}|\left(\mathrm{f}_{1_{\psi}}^{2}+\mathrm{g}_{1_{\psi}}^{2}\right)^{1 / 2}, \quad \Phi-1,
\end{gathered}
$$

em que a última é deduzida da eq. (4.12) em combinação com (4.16). Os valores $\sigma(1, \psi)$ são estritamente positivos por força do que se convencionou para os potenciais do emissor e coletor. Dada a possibilidede de se poder considerar formas bastante gerais para $\mathrm{F}(\psi,|\mathrm{E}|, 1 / \sigma), \sigma(1, \psi)$ poderia ser especificado diretamente de (4.17b) tomando $F(\psi,|E|, 1 / \sigma) \equiv \sigma_{0}(\psi)-\sigma$, ou então de (4.17c), caso se prescrevesse - campo no emissor. A primeira alternativa é o procedimento explicitamente adotado pelos autores.

Budd \& Wheeler [4] consideraram o modelo matemático de um domínio simplesmente conexo que pode acomodar ao mesmo tempo a geometria planar e outros sistemas menos convencionais de dois eletródios. O primeiro caso permite uma comparação direta de resultados computados com a solução unidimensional exata descrita pelas equações (2.13). O segundo proporciona uma experimentação numérica mais sistemática, em geometria menos trivial, de algumas situações interessantes, tais como testar diferentes parametrizações do contorno $\Phi-1$ (ou $\partial \Omega_{0}$ na Fig. -4.1 ), formas e magnitudes diferentes da carga injetada $\left(\sigma_{0}(\psi)\right)$, etc.

O modelo acima é definido a partir das eqs. (4.16) pondo-se (a) $\underline{\mathrm{H}(\mathrm{x}, \mathrm{y}) \equiv x-1=0}$ e (b) $\underline{\mathrm{x}=\mathrm{f}_{1}}(\psi) \equiv \mathrm{f}(\psi) \rightarrow 0 \underline{\text { quando }} \underline{\psi \rightarrow \psi_{0}} \underline{\mathrm{e}} \psi \rightarrow \psi_{1}$. (a) corresponde a um eletródio plano localizado em $x=1$; se $f(\psi) \equiv 0$, (b) dá um segundo eletródio plano em $x=0$ e o conjunto é, obviamente, a geometria planar; se $f(\psi)$ é doutra 
forma, (b) dá um segundo eletródio (emissor) não plano mas que ultimamente se torna paralelo ao primeiro. Assim, considerando a parametrização $y=g_{1}(\psi) \equiv \psi$ e particularizando $G_{2}(x, y)$ e $G_{3}(x, y)$ à forma $G_{2,3}(x, y) \equiv x-G(\Phi)=0$, o problema para $x, y, \sigma$ e $a(\psi)$ definido pelas eqs. (4.11) satisfaz às condições de contorno (4.16) agora reescritas como

$$
\begin{aligned}
\{\mathrm{x}, \mathrm{y}\}=\{\mathrm{f}(\psi), \psi\}, \quad \sigma(\Phi, \psi)=\sigma_{0}(\psi) \quad \text { em } \quad \Phi=1 ; \\
\mathrm{x}=1 \quad \text { em } \quad \Phi=0 ; \\
\mathrm{x}=\mathrm{G}(\Phi) \quad \text { em } \quad \psi=\psi_{0}, \psi_{1} .
\end{aligned}
$$

É importante observar que, se nestas geometrias $\sigma_{0}(\psi) \rightarrow \sigma_{0}-c t e$, quando $\psi \rightarrow \psi_{0}$ e $\psi \rightarrow \psi_{1}$, então na última condição de contorno $G(\Phi)$ é a solução unidimensional em (2.13c,d). Seja como for, as eqs. (4.11a) juntamente com as condições acima definem agora um problema eliptico com condições de contorno do tipo de Dirichlet para $\mathrm{x}(\Phi, \psi)$ (ver eq. (4.18b)).

Neste tipo particular de geometrias e ndiçōes de contorno, os autores estabelecem o seu esquema numérico na implementação do seguinte algorítmo convergente.

Algoritmo I. Sejam $x_{n}$, an $e$ $\sigma_{n}(n \geq 1)$ os n-ésimos valores iterados de $\mathrm{x}$, a $\sigma$, respectivamente.

1. Inicialmente supōem-se, tentativamente, $x_{0}(\Phi, \phi)$ e $a_{0}(\not)$ tal que $\sigma_{0}(1, \phi) \dot{e}$ consistente com $(4.17 \mathrm{~b}, \mathrm{c})$.

2. Resolve-se para ô a seguinte equação ordinária, ${ }^{4}$

$$
\hat{\sigma}_{*}=-\left(x_{*}^{2}+x_{\psi}^{2} / a^{2} \sigma_{n-1}^{2}\right)
$$

com $\hat{\sigma}(1, \not)$ determinado por $(4.17 b, c)$.

3. Resolve-se a equação diferencial parcial

$$
\left(a(\boldsymbol{\phi}) \sigma \hat{\mathbf{x}}_{\psi}\right)_{\xi}+\left(\hat{\mathbf{x}}_{\phi} / a(\boldsymbol{p}) \sigma\right)_{\phi}=0
$$

que resulta do desacoplamento das eqs. (4.16a) para $\hat{x}$. 
4. Atualizam-se $\sigma$ e $\times$ por

$$
\sigma_{n}=\omega \sigma_{n-1}+(1-\omega) \hat{\sigma}, \quad x_{n}=\omega x_{n-1}+(1-\omega) \hat{x}
$$

onde $\omega$ é um parâmetro de relaxaçāo adequado.

5. Repetem-se os passos $\underline{2}$ a $\underline{4}$ até a convergência de $\times e \sigma$.

6. Calcula-se e atualiza-se a(p) por, respectivamente,

$$
\hat{a}=\left(\left.\left.x_{s}\right|_{\varepsilon=1} \sigma\right|_{s=1}\right)^{-1}, \quad a_{n}=\omega a_{n-1}+(1-\omega) \hat{a} .
$$

\section{Repetem-se os passos $\underline{2}$ a $\underline{6}$ até a convergência.}

A equação para â( () , em (4.18d), foi obtida da primeira das eqs. (4.11a) calculada sobre o emissor ( $\Phi=1)$ na parametrização $y=g_{1}(\psi) \equiv \psi$ (ver eq. (4.16)).

Antes de prosseguir a implementação deste algoritmo, três observações devem ser feitas: (a) não há indicação explicita dos autores sobre como proceder o cálculo de $y(\Phi, \psi)$. É certo, porém, que pode ser feito por meio da equação diferencial

$$
y_{\phi}=-x_{p} /(a(\psi) \sigma)
$$

com $y(\Phi, \psi)$ satisfazendo às condições de contorno

$$
\mathrm{y}=\psi, \quad \Phi=1 \quad \text { e } \quad y_{\phi}=0, \quad \Phi=0
$$

(b) geometrias simplesmente conexas mais gerais, com fronteiras em $\psi_{0}$ e $\psi_{1}$ dadas por linhas de campo, exigem um tratamento numérico diferente. Os autores sugerem idéias sobre um algoritmo mas não consideram estes casos na prática; (c) também não deixam clara a maneira como procederam a discretização das eqs. $(4.18 \mathrm{a}, \mathrm{d}, \mathrm{e})$. As formas $(4.19 \mathrm{~b}-22)$ expostas aqui são a nossa interpretacão.

Para implementar o Algoritmo I, as equações a derivadas parciais (4.18a,b,d,e) são adequadamente aproximadas por equações de diferenças finitas e, então, resolvidas nos pontos de uma grade retangular uniforme, tal como a esquematizada na Fig. -4.2 . 


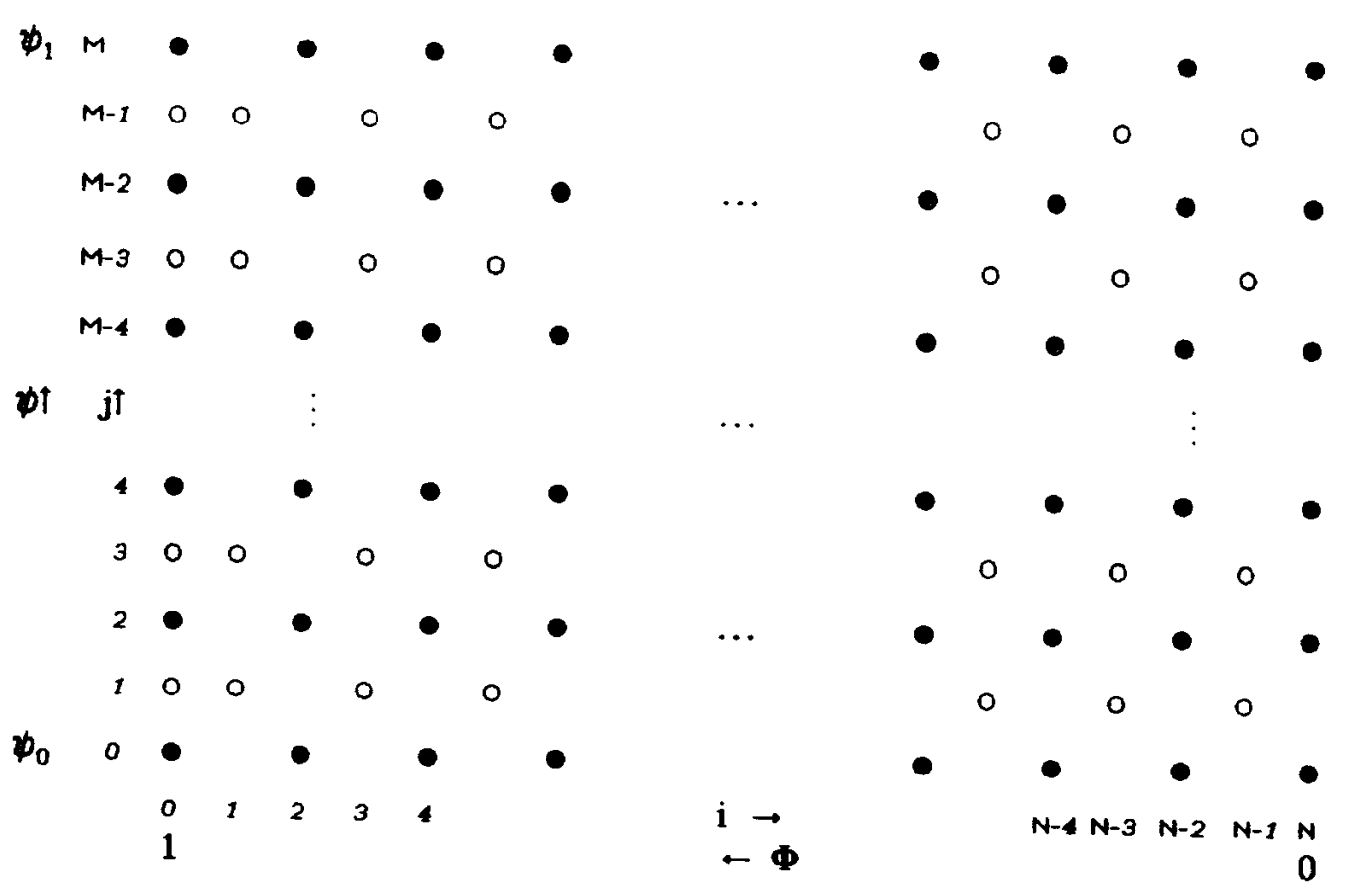

FIG.-4.2 Grade de discretização das eqs. (4.18a,b,d,e). Os nodos principais "-" formam um arranjo de corpo ajustado de espaçamento $h,(h=|\Delta \Phi|=|\psi|)$ cujas malhas estāo centradas pelos nodos secundários "o".

A discretização de $(4.18 b)$ emprega diferenças centrais para todas as derivadas ali presentes e é processada sobre a molécula da Fig. - 4.3.

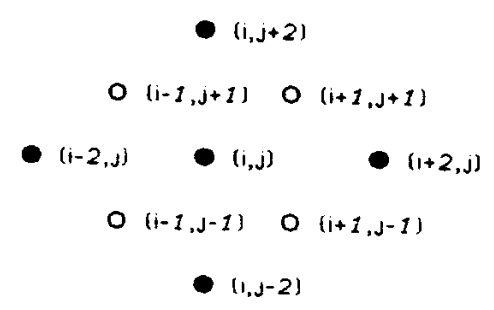

FIG. - 4.3 Molécula para aproximar a eq. (4.18b) por diferenças finitas.

Se $\hat{x}(i, j)$ é uma aproximacão de $x(\Phi, \psi)$ nos nodos principais “ $e$ " e $a(j)$ e $\sigma(i, j)$ as de $a(\psi)$ e $\sigma(\Phi, \psi)$ nos nodos secundários " 0 ", esta discretizacão produz o seguinte 
sistema de equações algébricas

$$
\begin{gathered}
\alpha_{4}(i-1, j) \hat{x}(i-2, j)+\alpha_{3}(i, j-1) \hat{x}(i, j-2)+A(i, j) \hat{x}(i, j)+ \\
+\alpha_{2}(i, j+1) \hat{x}(i, j+2)+\alpha_{1}(i+1, j) \hat{x}(i+2, j)=0
\end{gathered}
$$

com

$$
\begin{gathered}
\alpha_{1}(i+1, j)=\frac{1}{2 h^{2}}[a(j-1) \sigma(i+1, j-1)+a(j+1) \sigma(i+1, j+1)], \\
\alpha_{2}(i, j+1)=\frac{2}{h^{2}}\{a(j+1)[\sigma(i-1, j+1)+\sigma(i+1, j+1)]\}^{-1}, \\
\alpha_{3}(i, j-1)=\frac{2}{h^{2}}\{a(j-1)[\sigma(i-1, j-1)+\sigma(i+1, j-1)]\}^{-1}, \\
\alpha_{4}(i-1, j)=\frac{1}{2 h^{2}}[a(j-1) \sigma(i-1, j-1)+a(j+1) \sigma(i-1, j+1)], \\
A(i, j)=-\left[\alpha_{1}(i+1, j)+\alpha_{2}(i, j+1)+\alpha_{3}(i, j-1)+\alpha_{4}(i-1, j)\right], \\
i=2(N-2) 2, \quad j=2(M-2) 2,
\end{gathered}
$$

que deve ser resolvido em conjunto com as condições de contorno

$$
\hat{\mathbf{x}}(0, j)=f\left(\psi_{0}+\frac{j h}{2}\right), \quad \hat{x}(N, j)=1, \quad \hat{x}(i, 0)=G\left(1-\frac{i h}{2}\right)=\hat{x}(i, M)
$$

A solução numérica da equação ordinária (4.18a) emprega a regra trapezoidal e diferenças centrais (quando possivel) para aproximar as derivadas $x_{\phi}$ e $x_{\not p}$.

(a)

(b)

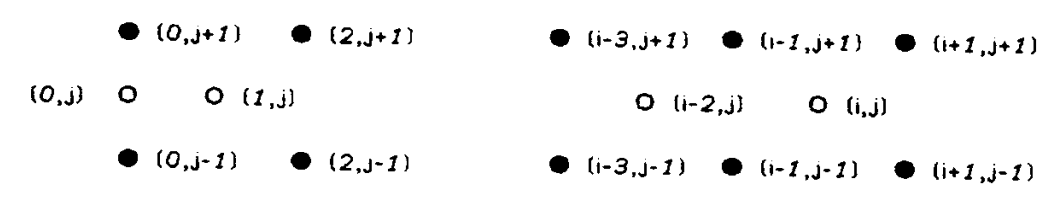

FIG. - 4.4 Moléculas de diferença para aproximar a eq. (4.18a) - (a) próximo do emissor $(\Phi=1)$ e (b) no corpo do dominio (Fig. -4.2). 
A discretização de (4.18a) é tomada sobre as moléculas da Fig. -4.4 para dar as fórmulas

$$
\sigma(1, j)=\sigma(0, j)+\frac{h}{2}\left[x_{\phi}^{2}(1, j)+\frac{x_{p}^{2}(1, j)}{a^{2}(j) \sigma_{n-1}^{2}(1, j)}\right]
$$

com

$$
\begin{aligned}
& x_{\phi}(1, j)=-\frac{1}{2 h}[x(2, j-1)+x(2, j+1)-x(0, j-1)-x(0, j+1)], \\
& x_{p}(1, j)=\frac{1}{2 h}[x(2, j+1)+x(0, j+1)-x(2, j-1)-x(0, j-1)],
\end{aligned}
$$$$
\sigma(0, \mathrm{j})=\sigma_{0}\left(\psi_{0}+\mathrm{jh} / 2\right)
$$

$$
j=1(M-1) 2 \text {. }
$$

$$
\hat{\sigma}(i, j)=\hat{\sigma}(i-2, j)+\frac{h}{2}\left[x_{\phi}^{2}(i, j)+\frac{x_{\not p}^{2}(i, j)}{a^{2}(j) \sigma_{n-1}^{2}(i, j)}+x_{\phi}^{2}(i-2, j)+\frac{x_{\not}^{2}(i-2, j)}{a^{2}(j) \sigma_{n-1}^{2}(i-2, j)}\right]
$$

com

$$
\begin{aligned}
& x_{\phi}(i, j)=-\frac{1}{2 h}[x(i+1, j-1)+x(i+1, j+1)-x(i-1, j-1)-x(i-1, j+1)] \\
& x_{\phi}(i-2, j)=-\frac{1}{2 h}[x(i-1, j-1)+x(i-1, j+1)-x(i-3, j-1)-x(i-3, j+1)], \\
& x_{\phi}(i, j)=\frac{1}{2 h}[x(i+1, j+1)+x(i-1, j+1)-x(i+1, j-1)-x(i-1, j-1)] \\
& x_{\phi}(i-2, j)=\frac{1}{2 h}[x(i-1, j+1)+x(i-3, j+1)-x(i-1, j-1)-x(i-3, j-1)] \\
& i=3(N-1) 2, \quad j=1(M-1) 2 .
\end{aligned}
$$

que devem ser resolvidas sobre cada linha nodal secundária j-cte.

(a)

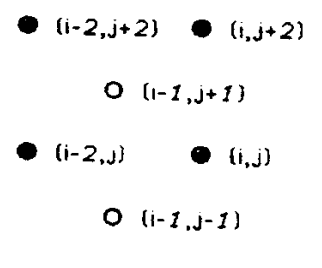

- $(i-2, j-2) \bullet(1, j-2)$ (b)

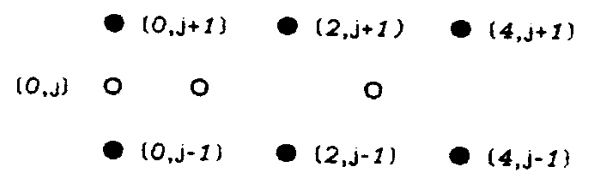

FIG.-4.5 Moléculas de diferença para aproximar (a) a eq. $(4.18 \mathrm{e})$ e (b) a eq. $(4.18 \mathrm{~d})$. 
Uma discussão semelhante se aplica às equações restantes. Tendo-se por base as moléculas de discretização das Fig.-4.5, (4.18d) é aproximada pelas equações

$$
a(j)=\left[\sigma(0, j) x_{\phi}(0, j)\right]^{-1}
$$

com

$$
\begin{gathered}
x_{\phi}(0, j)=-\frac{1}{4 h}\{4[x(2, j+1)+x(2, j-1)]-3[x(0, j-1)+x(0, j+1)]-[x(4, j+1)+x(4, j-1)]\}, \\
j=1(M-1) 2,
\end{gathered}
$$

e por último, no passo extra, (4.18e) é aproximada por

$$
y(i, j)=y(i-2, j)+h x_{p}(i-1, j) /\left\{\frac{1}{2}[a(j+1) \sigma(i-1, j+1)+a(j-1) \sigma(i-1, j-1)]\right\},
$$

com

$$
\begin{gathered}
x_{\phi}(i-1, j)=\frac{1}{4 h}[x(i, j+2)+x(i-2, j+2)-x(i, j-2)-x(i-2, j-2)], \\
j=2(M-2) 2, \quad i-2(N-2) 2 . \\
y(N, j)=y(N-2, j), \quad y(0, j)=y(0,0)+j h / 2=\psi_{0}+j h / 2 .
\end{gathered}
$$

Quando o sistema de equações (4.19a) é posto na forma matricial $\mathrm{Mx-b}$, $M$ resulta uma matriz esparsa $\left[\left(\frac{N-2}{2}\right)\left(\frac{M-2}{2}\right)\right] \times\left[\left(\frac{N-2}{2}\right)\left(\frac{M-2}{2}\right)\right]$, com $\left[\frac{5}{4} N M-\frac{7}{2}(N+M)+9\right]$ elementos não nulos distribuidos sobre cinco diagonais apenas:- a diagonal principal, as duas secundárias principais e duas outras que se iniciam nas posições $(1, N / 2)$ e $(N / 2,1)$ quando a grade na Fig. -4.2 é varrida da esquerda para a direita e de baixo para cima. Para resolver este sistema os autores empregaram o "Procedimento Fortemente Implicito" de Stone, conforme descrito por Jacobs [25].

Baseados na hipótese de que $x(\Phi, \psi)$ seja uma função razoavelmente suave de $(\Phi, \psi)$, Budd \& Wheeler [4] fazem uma breve análise para prever que o seu esquema calcula $x(\Phi, \psi)$ e $\sigma(\Phi, \psi)$ com uma precisão de segunda ordem $\left(O\left(h^{2}\right)\right)$. Isto foi verificado em experimentações numéricas nas quais se computa uma aproximação para a solução unidimensional exata. Além destas, outras experiências numéricas foram realizadas para se firmar a avaliação de que o algoritmo é de 
fato rapidamente convergente, tanto para um certo conjunto de parâmetros de relaxacao $\omega$, quanto para diferentes parametrizações da fronteira $\Phi=1$. Em particular, considera-se 0 caso em que $f_{1}(\phi)=0.5 \exp \left\{-0.25 \psi^{2}\right\}$ e $\sigma(1, \psi)=1-0.9 \exp (-|\psi|) \sin (3 \psi)$, cujos resultados estão mostrados na Fif. -4.6 para uma comparação futura.
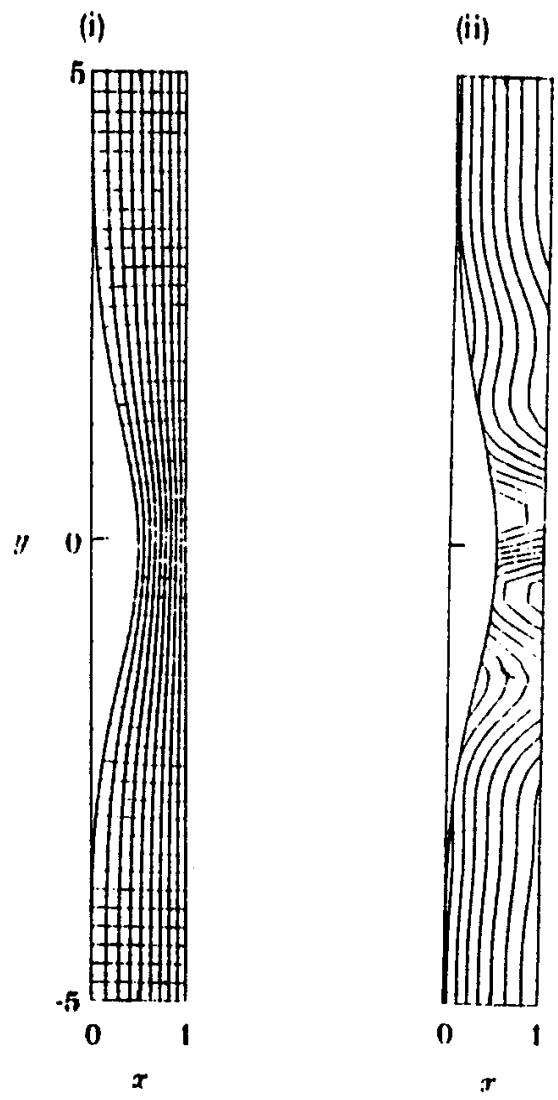

FIG. - 4.6 O caso $f_{1}(\psi)-0.5 \exp \left(-0.25 \psi^{2}\right), \sigma(1, \psi)-1-0.9 \exp (-|\psi|) \sin (3 \psi)$ : (i) mostra linhas de campo e equipotenciais e (ii) as curvas de nivel de $\sigma(\Phi, \psi)$. Extraido de Budd \& Wheeler [4], pp. 401. 


\section{CAPITULOV}

\section{Injeção Descontínua de Carga Espacial em Geometria Planar}

\section{1 - Introdução}

Neste capitulo nós empregamos o Método de Budd \& Wheeler [4] na determinação de uma solução numérica para o sistema das equações (4.11) em uma geometria simplesmente conexa, cujas fronteiras e/ou condições de contorno estão parcialmente definidas pelas seguintes equações (ver notação na sec.4.3.2, cap.IV):

$$
\begin{gathered}
\mathrm{x}=\mathrm{f}_{1}(\psi) \equiv 0, \quad \Phi-1 ; \quad \mathrm{H}(\mathrm{x}, \mathrm{y}) \equiv \mathrm{x}-1=0, \quad \Phi-0 \\
\mathrm{y}=\mathrm{g}_{1}(\psi) \equiv \psi, \quad \Phi-1 ; \quad \mathrm{y}_{-}=0, \quad \Phi=0 \\
\mathrm{~F}(\boldsymbol{\Phi},|\mathrm{E}|, \sigma) \equiv \sigma(\Phi, \psi)-\sigma_{0}=0, \quad 0 \leq \psi \leq+\psi_{0}, \quad \Phi-1 ; \\
\mathrm{F}(\boldsymbol{\psi},|\mathrm{E}|, \rho) \equiv \rho(\Phi, \psi)-0, \quad-\psi_{0} \leq \psi<0, \quad \Phi-1 . \\
\mathrm{G}_{3}(\mathrm{x}, \mathrm{y}) \equiv \mathrm{x}-(1-\Phi)=0, \quad \psi--\psi_{0} . \\
\mathrm{G}_{2}(\mathrm{x}, \mathrm{y}) \equiv \mathrm{x}-\frac{1}{2} \sigma_{0} \mathrm{E}_{0}\left(\left[1+\frac{3}{\sigma_{0} \mathrm{E}_{0}^{2}}(1-\Phi)\right]^{2 / 3}-1\right\}=0, \quad \psi=+\psi_{0} ; \\
\mathrm{E}_{0}^{3}+\left(\frac{2}{\sigma_{0}}-1\right) \mathrm{E}_{0}^{2}+\frac{4}{3 \sigma_{0}^{2}} \mathrm{E}_{0}-\frac{3}{2 \sigma_{0}}=0 .
\end{gathered}
$$

Em (5.1a) definimos um par de eletródios planos, paralelos, com a disposição espacial mostrada na Fig.-5.1(a), enquanto (5.1b) mostra nossa escolha particular de parametrização da superfície emissora (parcialmente emissora), significando dizer que a linha de campo que a intersecta (superficie) no ponto $\left(x^{*}, y^{*}\right)$, tal que $\left(x^{*}, y^{*}\right)-\left(1, \psi^{*}\right)$, é precisamente a curva $\left\{x\left(\Phi, \psi^{*}\right), y\left(\Phi, \psi^{*}\right)\right\} \operatorname{com} 0 \leq \Phi \leq 1$.

O problema em definição se distingue dos já formulados nessa geometria, pela forma particular segundo a qual a carga espacial é injetada no espaço inter-eletródios. Segundo (5.1c)-em que $\sigma_{0}$ e $\psi_{0}$ são constantes 
positivas - ao invés de se dar ao longo de toda a extensão do eletródio ativo, a emissão é descontínua, só ocorrendo na região compreendida entre $y=0$ e $y=+y_{0}$ ou, no plano $(\Phi, \psi)$, conforme a Fig. $-5.1(b)$, no segmento $0 \leq \psi \leq+\psi_{0}$ da equipotencial $\Phi=1$.

- carater hiperbólico das equações de carga espacial permite estabelecer [26] um importante resultado: - em uma geometria simplesmente conexa ou uniconexa, se a densidade de carga espacial é nula em alguma região do eletródio emissor, então $\rho \equiv 0$ sobre as linhas de campo que dela emanam e assim regiöes de carga espacial nula estarão presentes no espaço entre os eletródios. Consequentemente a emissão descrita em (5.1c) dá origem a duas regiões distintas no domínio considerado.
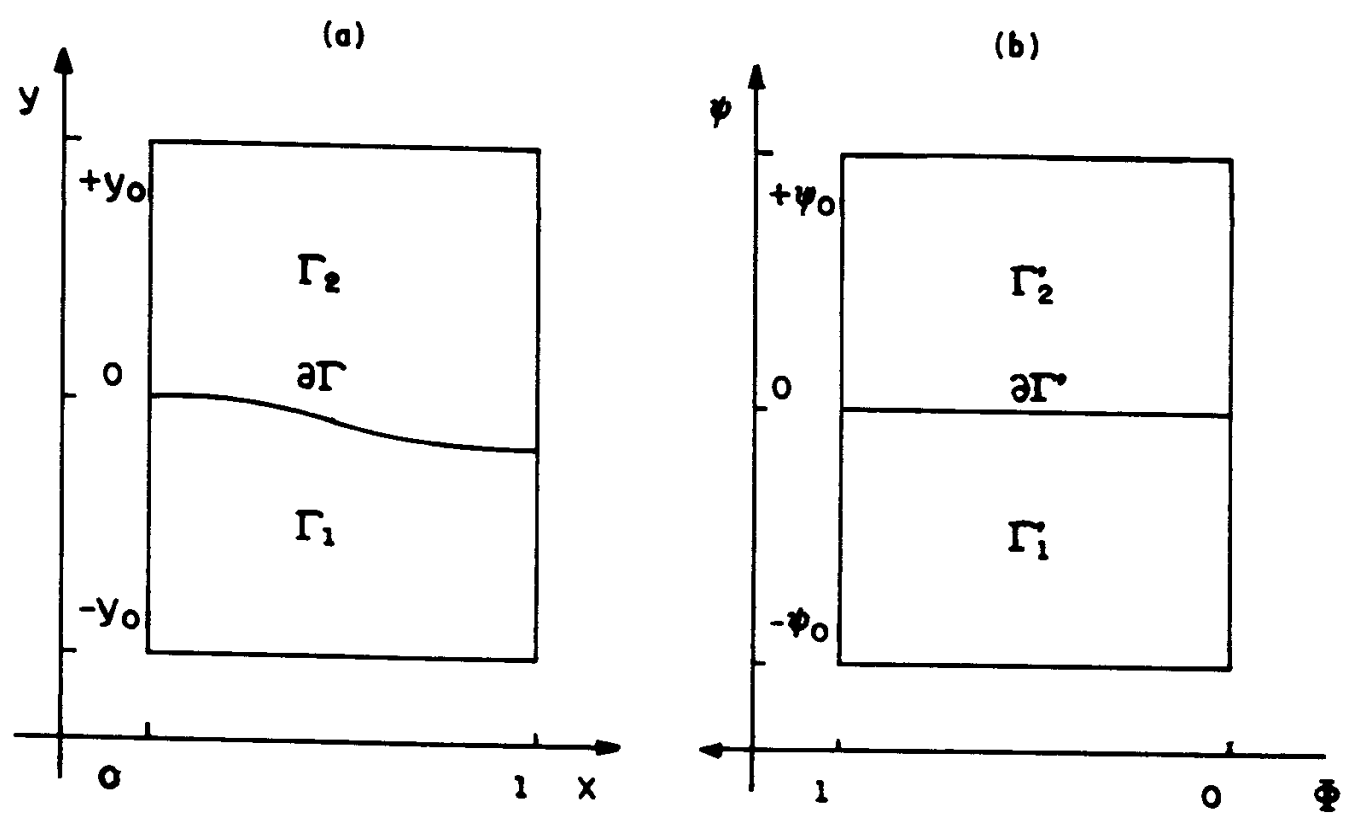

FIG. -5.1 (a) Sistema de dois eletródios planos, paralelos, com emissão
de carga restrita à extensão $0 \leq y \leq+y_{0}, x=0$ do eletródio ativo; (b)
mapeamento do espaço inter-eleródios no plano $(\Phi, \psi)$.

Em uma delas, $\Gamma_{1}$ - que poderiamos chamar de "região de Laplace" - a carga espacial é nula e o campo é descrito apenas pelas equaçōes elípticas (4.11a) com $\sigma(\Phi, \psi) \equiv 1$. A outra, $\Gamma_{2}$ - a "região de Poisson" - é onde ocorre o fluxo de carga propriamente dito.

Como mostramos na Fig. $-5.1(a)$, as regiōes $\Gamma_{1}$ e $\Gamma_{2}$ estão separadas ao 
longo da curva continua $\partial \Gamma$. Esta deve coincidir com a linha de campo emanada do último ponto de emissão de carga espacial, $(x, y)=(0,0)$, e cuja determinacão constitui o probleme central deste capitulo.

Com relação às eqs. (5.1d) e (5.1e), a nossa suposição é a de que, em pontos distantes da fronteira interna $\partial \Gamma$, o potencial elétrico se torne linear na região de Laplace, como deveria ser na ausência total de carga espacial e, na de Poisson, seja a solução unidimensional exata das equações de carga espacial (como seria caso a injeção fosse uniforme em todo o emissor).

A existência de duas regiōes distintas no dominio considerado sugere, de imediato, um esquema natural de solução:

1. Dado $x(\Phi, \psi)$ sobre a fronteira comum $\partial \Gamma$, resolvem-se iterativamente as equações de 'carga espacial' em cada uma das regiōes $\Gamma_{1} e$ $\Gamma_{2}$, como se fossem dominios simples isolados, para determinar $x_{1}, y_{1}$ e $a_{1}(\psi)$ em $\Gamma_{1}$ e $x_{2}, y_{2}, a_{2}(\psi)$ e $\sigma_{2}$ em $\Gamma_{2}$.

2. Dadas as soluçōes em $\Gamma_{1} \in \Gamma_{2}$, buscam-se condições de contorno apropriadas para determinar $x(\Phi, \psi)$ e $y(\Phi, \psi)$ sobre $\partial \Gamma$.

Uma implementação adequada deste esquema ${ }^{1}$ deve considerar inicialmente as condições que tornariam possivel juntar em $\partial \Gamma$ a solução de fluxo estacionário de carga por um lado, com a solução de campo harmônico por outro. Estas condições são deduzidas na sec.5.1 a partir da continuidade do potencial e do vetor campo elétrico através de ar. Nessa secção nós também discutimos uma forma aproximada para $x(\Phi, \psi)$ sobre $\partial \Gamma$. Com ela, não só completamos as condições de contorno para o tratamento individualizado dos subdomínios $\Gamma_{1}$ e $\Gamma_{2}$ na sec.5.2, como damos inicio às iterações necessárias à convergência do esquema.

Para tratarmos os problemas numéricos específicos de $\Gamma_{1}$ e $\Gamma_{2}$, nós adotamos um procedimento análogo ao que Budd \& Wheeler [4] (B\&W) dispensaram a um dominio simplesmente conexo. Mais precisamente, nós usamos o seu (deles) Algoritmo I (sec.4.4, cap.IV) com algumas modificações introduzidas nos passos $\underline{1} \mathrm{e}$ 2 e no método de relaxação empregado. Estas modificações são discutidas 'Agradecemos aquí as sugestōes feitas pelo Prof. José Alberto Cuminato (ICMSC-USP-S. CARLOS). 
juntamente com a definição do nosso algorítmo na sec.5.3.

Na consideracãa isolada de $\Gamma_{2}$, a simples adaptação das condições de contorno nos permite fazer uma variedade de experiências numéricas diferentes. Numa primeira etapa nós realizamos apenas duas:- uma, para reproduzir qualitativamente os resultados de B\&W, nas condições da Fig.-4.6; a outra, para computar os dados com os quais confrontamos a solução desenvolvida no cap.III (sec.3.5, Fig.-3.8(c)). Porém no capitulo VII voltaremos a repetí-las em condições diferentes.

Os nossos resultados numéricos são apresentados e discutidos no proximo capitulo.

\section{2 - As Condiçōes de Contorno em ar}

No plano $(\Phi, \psi)$, como mostramos na Fig.-5.1(b), a transformação hodográfica de $\partial \Gamma$ (ar') está definida pela curva de nivel $\psi=0$. Se $x(\Phi, 0)$ e y $(\Phi, 0)$, com $0 \leq \Phi \leq 1$, definem as coordenadas de um ponto de $\partial \Gamma$ na transformação inversa, então a continuidade do potencial por todos os pontos do domínio permite estabelecer as seguintes condições de contorno [26] em $\partial \Gamma$ :

$$
\begin{aligned}
& \lim _{\psi_{1} \rightarrow 0} x_{1}\left(\Phi, \psi_{1}\right)=\lim _{\psi_{2} \rightarrow 0} x_{2}\left(\Phi, \psi_{2}\right)=x(\Phi, 0), \\
& \lim _{\psi_{1} \rightarrow 0} y_{1}\left(\Phi, \psi_{1}\right)=\lim _{\psi_{2} \rightarrow 0} y_{2}\left(\Phi, \psi_{2}\right)=y(\Phi, 0),
\end{aligned}
$$

ambas com

$$
0 \leq \Phi \leq 1
$$

Nestas identidades, bem como nas seguintes, os sub-indices $\underline{1}$ e $\underline{2}$ significam que as correspondentes variáveis tomam seus valores nos subdominios $\Gamma_{1}$ e $\Gamma_{2}$, respectivamente.

Por outro lado, como ef etivamente $\Gamma_{1}$ e $\Gamma_{2}$ não constituem dois meios de diferentes constantes dielétricas, o vetor campo elétrico deve também ser continuo através de $\partial \Gamma$. Desta propriedade resultam mais duas condicões de 
contorno [26], a saber, com $0 \leq \Phi \leq 1$,

$$
\begin{aligned}
& \lim \left[\mathrm{x}_{\boldsymbol{\psi}_{1}}\left(\Phi, \psi_{1}\right) / \mathrm{a}_{1}\left(\psi_{1}\right)\right]=\lim \left[\mathrm{x}_{\mathrm{p}_{2}}\left(\Phi, \psi_{2}\right) /\left(\mathrm{a}_{2}\left(\psi_{2}\right) \sigma_{2}\left(\Phi, \psi_{2}\right)\right)\right]=\mathrm{y}_{\boldsymbol{\Phi}}(\Phi, 0), \\
& \psi_{1} \rightarrow 0 \quad \psi_{2} \rightarrow 0
\end{aligned}
$$

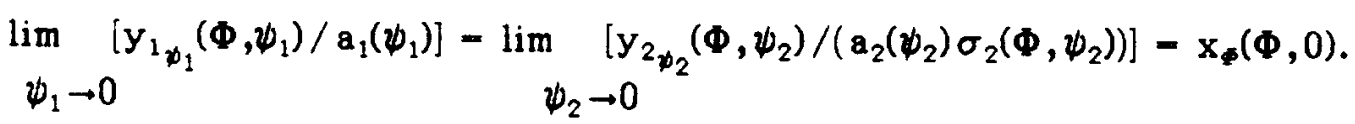

As condições de contorno (5.2) são satisfeitas por construção, já que a curva $\partial \Gamma$ define necessariamente a fronteira entre as duas regiōes (rever a Fig. -5.1). Torna-se portanto evidente, que somente as eqs. (5.3) são susceptiveis de implementar o passo $\underline{2}$ do esquema de cálculo em consideração.

Para implementar o passo 1 , nós vamos agora deduzir uma solução tentativa para $x(\Phi, 0)$ sobre $\partial \Gamma$. Observemos então, que se não houvesse carga espacial no dominio, a solução seria exatamente o resultado dado em (5.1d). Por outro lado, se a presença da carga se desse pela injeção uniforme $\left(\rho(1, \emptyset)=1 / \sigma_{0}=c t e.\right)$ em toda a extensão da fonte, (5.1e) daria a forma exata de $x(\Phi, 0)$. Esses resultados definem os limites para $x(\Phi, 0)$ na coexistência das duas situações, dentro dos quais a nossa solução computada deve se encontrar. Portanto, uma aproximação razoável para $x(\Phi, 0)$ parece ser a média das funções em (5.1d) e (5.1e), ou seja,

$$
x(\Phi, 0)=\frac{1}{2}(1-\Phi)+\frac{1}{4} \sigma_{0} E_{0}\left\{\left[1+\frac{3}{\sigma_{0} E_{0}^{2}}(1-\Phi)\right]^{2 / 3}-1\right\}
$$

com

$$
E_{0}^{3}+\left(\frac{2}{\sigma_{0}}-1\right) E_{0}^{2}+\frac{4}{3 \sigma_{0}^{2}} E_{0}-\frac{3}{2 \sigma_{0}}=0 \quad \text { e } \quad 0 \leq \Phi \leq 1
$$

Vale a pena observar que na prática a função em (5.4) é de fato uma excelente aproximação para as soluçōes computadas de $x(\Phi, 0)$, e que portanto deve levar a uma considerável redução do número de iterações necessárias à convergência. 


\title{
5.3 -Abordagem de $r_{1}$ e $\Gamma_{2}$ como Dominios Isolados
}

\begin{abstract}
Nesta secção nós reunimos os sistemas de equações e as condições de contorno apropriadas para tratar as regiões $\Gamma_{1}$ e $\Gamma_{2}$ como dois domínios simplesmente conexos independentes, conforme planejado no passo 1 do nosso esquema numérico.
\end{abstract}

\subsection{1 - Região de Laplace}

Como a carga espacial é nula em $\Gamma_{1}$, as equações do problema nesta região se reduzem ao sistema elíptico

$$
\begin{aligned}
& y_{b}=b(\psi) x_{\phi}, \\
& x_{\phi b}=-b(\psi) y_{\phi},
\end{aligned}
$$

que resultam imediatamente das eqs. (4.11) com a adaptação necessária $\sigma(\Phi, \psi) \equiv 1$ e a mudança de notação $a(\psi) \rightarrow b(\psi)$. Efetivamente não se tratando mais de um problema de carga espacial, as condiçōes de contorno se referem tão somente à parametrização das fronteiras de $\Gamma_{1}$. São elas, coerentemente com as eqs. (5.1),

$$
\left.\begin{array}{rl}
\mathrm{x}=0, \quad \Phi=1 ; \quad \mathrm{x}=1, \quad \Phi=0 ; \\
\mathrm{x}=1-\Phi, \quad \psi=-\psi_{0} ; \quad \mathrm{x}=\mathrm{x}(\Phi, 0), \quad \psi=0^{-} ; \\
\mathrm{y}=\psi, \quad \Phi=1 ; \quad \mathrm{y}_{\phi}=0, \quad \Phi=0 ;
\end{array}\right\}
$$

onde $0^{-}$denota $\circ$ limite superior (Fig.-5.1(a)) da região de Laplace junto à fronteira $\partial \Gamma$. É importante observar que na última condição de $(5.6 a), x(\Phi, 0)$ é uma funcão que está prescrita em $\partial \Gamma$, seja inicialmente pela fórmula em (5.4), seja por sucessivos valores iterados como será mostrado depois.

Na prática, aliás como sugerem B\&W no seu Algoritmo I (sec.4.4, 
cap.IV), usamos também a formulação que consiste em colocar o problema em três estágios de cálculo diferentes: $1^{\circ}$ - determinar $x(\Phi, \psi)$, ainda em função de $b(\psi)$, através da equação

$$
\left[b(\psi) x_{\psi}\right]_{\phi}+\left[x_{\psi} / b(\psi)\right]_{\psi}=0
$$

juntamente com as condições de contorno em (5.6a). Esta equação é obtida por eliminação das derivadas $y_{\varnothing}$ e $y_{\downarrow}$ entre as duas de (5.5); $2^{\circ}$ - calcular $b(q)$, em função de $x$, por meio da identidade

$$
b(\psi)=\left(x_{\Phi}(1, \psi)\right)^{-1}
$$

que se deduz substituindo $y(1, \psi)=\psi$ na primeira equação de $(5.5) ; 3^{\circ}$ - obter $y(\Phi, \psi)$, em função de $b(\psi)$ e $x$, por meio da integração ordinária da última equação de (5.5) ou seja, de

$$
y_{*}=-x_{p} / b(\psi)
$$

com a condição inicial $y(1, \psi)=\psi$.

Nós iniciamos os cálculos em $\Gamma_{1}$ usando as duas seguintes funções tentativa para $x(\Phi, \psi)$ e $b(\psi)$, respectivamente,

$$
x(\Phi, \psi)-\gamma(\Phi, \psi)\left(\psi+\psi_{0}\right)+(1-\Phi), \quad 0<\Phi<1, \quad-\psi_{0}<\psi<0
$$

com

$$
\begin{gathered}
\gamma(\Phi, \psi)=\left\{\frac{1}{2} \sigma_{0} E_{0}\left\{\left[1+\frac{3}{\sigma_{0} E_{0}^{2}}(1-\Phi)\right]^{2 / 3}-1\right\}-(1-\Phi)\right\} / 2 \psi_{0} \\
E_{0}^{3}+\left(\frac{2}{\sigma_{0}}-1\right) E_{0}^{2}+\frac{4}{3 \sigma_{0}^{2}} E_{0}-\frac{3}{2 \sigma_{0}}=0 \\
b(\psi)=-1, \quad-\psi_{0}<\psi<0
\end{gathered}
$$

\subsection{2 - Região de Poisson}

No domínio representado por $\Gamma_{2} \circ$ problema está completamente definido pelas equações de carga espacial (4.11) juntamente com as condições de contorno abaixo, em que $\sigma_{0}$ é uma constante positiva. 


$$
\begin{gathered}
x=0, \quad \Phi=1 ; \quad x=1, \quad \Phi=0 ; \\
x=x(\Phi, 0), \quad \psi=0 ; \\
x=\frac{1}{2} \sigma_{0} E_{0}\left\{\left[1+\frac{3}{\sigma_{0} E_{0}^{2}}(1-\Phi)\right]^{2 / 3}-1\right\}, \quad \psi=+\psi_{0} . \\
\sigma(\Phi, \psi)=\sigma_{0}, \quad 0 \leq \psi \leq+\psi_{0}, \quad \Phi=1 \\
y=\psi, \quad \Phi=1 ; \quad y=0, \quad \Phi=0 .
\end{gathered}
$$

Outra vez a fronteira $\partial \Gamma$ se faz presente pela função prescrita $x(\Phi, 0)$. Todas as consideraçōes dispensadas a $x(\Phi, 0)$ na secção anterior também se aplicam aqui. Da mesma forma que antes, o cálculo em $\Gamma_{2}$ é posto em estágios distintos para $x, a(\psi)$, $\sigma$ e $y$, cujas fórmulas específicas estão expostas no Algorítmo II da sec.5.3 (ou no Algoritmo I do cap.IV).

O cálculo em $\Gamma_{2}$ é iniciado com três funçōes tentativa. Para $x(\Phi, \psi)$ nós usamos a mesma média ponderada descrita na eq. (5.10). Para $\sigma(\Phi, \psi)$ e a( () empregamos as suas expressões associadas à solução unidimensional exata, a saber,

$$
\begin{gathered}
\sigma(\Phi, \psi)=\sigma_{0}\left[1+\frac{3}{\sigma_{0} E_{0}^{2}}(1-\Phi)\right]^{1 / 3}, \quad 0<\Phi<1, \quad 0<\psi<+\psi_{0} ; \\
a(\psi)=-E_{0} / \sigma_{0}, \quad 0<\psi<+\psi_{0}
\end{gathered}
$$

valendo para as constantes $E_{0}$ e $\sigma_{0}$ a mesma relação em (5.10).

A consideração de $\Gamma_{2}$ com vistas à repetição dos resultados de $B \& W$, nas condições da Fig. -4.6 do cap.IV, e/ou para gerar os dados de comparação usados no cap.IIl, é enfocada no sentido mais geral no qual estaremos tratando com um dominio de fronteiras fixas, onde há carga espacial por toda parte. A alusão a $\Gamma_{2}$ deve então ser vinculada ao fato de que usaremos a mesma formulação básica e - mesmo procedimento numérico, enquanto tratamos $\Gamma_{2}$ como um domínio isolado. Assim, tendo em mente o primeiro objetivo, supomos que o dominio inteiro na Fig. - 5.1(a) seja uma única região de Poisson e que além disto, o eletródio emissor (emissor por inteiro) seja não plano com uma deformação gaussiana centrada em $y=0$. Nestas circunstâncias nós temos a estrutura de eletródios desejada e 
resolvemos as equações de carga espacial com as devidas condições de contorno, quais sejam,

$$
\begin{gathered}
x=0.5 \exp \left(-0.25 \psi^{2}\right), \quad \Phi=1 ; \quad x=1, \quad \Phi=0 ; \\
x=\frac{1}{2} \sigma_{0}^{\prime} E_{0}^{\prime}\left\{\left[1+\frac{3}{\sigma_{0}^{\prime} E_{0}^{2}}(1-\Phi)\right\}^{2 / 3}-1\right\}, \quad \psi= \pm \psi_{0} ; \\
\quad \sigma=1-0.9 \exp (-|\psi|) \sin (3 \psi), \quad \Phi=1 ; \\
y=\psi, \quad \Phi=1 ; \quad y_{\Phi}=0, \quad \Phi=0 .
\end{gathered}
$$

A constante $\sigma_{0}^{\prime}$ em (5.15a) é um dos dois valores de $\sigma$ em (5.15b) quando $\psi= \pm \psi_{0}$. Se $\psi_{0}>4, \quad \sigma_{0}^{\prime} \approx 1$ e é por esta razão que $x(\Phi, \psi)$ tem a forma da solução unidimensional exata nas fronteiras $\psi= \pm \psi_{0}$ (ver em (5.15a)).

Aqui o cálculo é iniciado com as seguintes funções tentativa:

$$
\begin{gathered}
x(\Phi, \psi)=\frac{1}{2} \sigma_{0}^{\prime} E_{0}^{\prime}\left\{\left[1+\frac{3}{\sigma_{0}^{\prime} E_{0}^{\prime 2}}(1-\Phi)\right]^{2 / 3}-1\right\}+0.5 \exp \left(-0.25 \psi^{2}\right) \Phi, \\
0<\Phi<1, \quad-\psi_{0}<\psi<+\psi_{0} . \\
\sigma(\Phi, \psi)=\sigma_{0}^{\prime}\left[1+\frac{3}{\sigma_{0}^{\prime} E_{0}^{\prime 2}}(1-\Phi)\right]^{1 / 3}[1-0.9 \exp (-|\psi|) \sin (3 \psi)], \\
0<\Phi<1, \quad-\psi_{0}<\psi<+\psi_{0} . \\
a(\psi)=-E_{0}^{\prime} / \sigma_{0}^{\prime}, \quad-\psi_{0}<\psi<+\psi_{0} .
\end{gathered}
$$

Finalmente, para resolver, pelo presente método, o problema colocada no cap.III, tomamos as condições de contorno coerentes com a estrutura de eletródios e o tipo de injeção de carga ali considerados:

$$
\begin{gathered}
\mathrm{x}=0, \quad \Phi=1 ; \quad \mathrm{x}=1, \quad \Phi=0 ; \\
\left.\mathrm{x}=\frac{1}{2} \sigma_{\mathrm{oi}} E_{\mathrm{oi}}\left\{\left[1+\frac{3}{\sigma_{0 \mathrm{i}} E_{\mathrm{oi}}^{2}}(1-\Phi)\right\}^{2 / 3}-1\right\}, \quad 0 \leq \Phi \leq 1, \quad \psi=-\psi_{0} ; \quad\right\} \\
\mathrm{x}=\frac{1}{2} \sigma_{\mathrm{oS}} E_{\mathrm{oS}}\left\{\left[1+\frac{3}{\sigma_{\mathrm{oS}} E_{\mathrm{oS}}^{2}}(1-\Phi)\right\}^{2 / 3}-1\right\}, \quad 0 \leq \Phi \leq 1, \quad \psi=+\psi_{0} \\
\sigma=\{2[1-\tan (0.5 \psi)]+0.5\}^{-1}, \quad \Phi=1 ;
\end{gathered}
$$




$$
y=\psi, \quad \Phi=1 ; \quad y_{\Phi}=0, \quad \Phi=0
$$

em que $\sigma_{i \mathrm{i}}$ e $\sigma_{0 \mathrm{~S}}$ são, respectivamente, os valores $\sigma\left(-\psi_{0}\right)$ e $\sigma\left(+\psi_{0}\right)$ dados por (5.19b). A relação entre $\sigma_{o i}$ e $E_{o i}\left(\sigma_{o S}\right.$ e $\left.E_{o S}\right)$ é do tipo que vimos usando já desde o começo do capitulo (ver eq. (5.1e)).

Tomamos para soluções tentativa iniciais:

$$
\begin{gathered}
x(\Phi, \psi)=\left(\left.x(\Phi)\right|_{w=-w_{C}}+\left.x(\Phi)\right|_{o=+w_{0}}\right) / 2, \quad 0<\Phi<1, \quad-\psi_{0}<\psi<+\psi_{0} ; \\
\sigma(\Phi, \psi)=\frac{1}{2}\left[\sigma\left(-\psi_{0}\right)+\sigma\left(+\psi_{0}\right)\right]\left[\left.\sigma(\psi)\right|_{\Phi=1}\right], \quad 0<\Phi<1, \quad-\psi_{0}<\psi<+\psi_{0} ; \\
a(\psi)=-\frac{1}{2}\left(E_{0 i} / \sigma_{0 i}+E_{0 S} / \sigma_{0 s}\right), \quad-\psi_{0}<\psi<+\psi_{0} .
\end{gathered}
$$

Nas duas últimas expressões $x(\Phi)$ e $\sigma(\Phi)$ estão definidas entre as eqs. (5.19).

\section{4 - Os Algoritmos para a Determinação de ar}

Antes mesmo de procedermos a construção de um algoritmo mais amplo, visando abranger a totalidade dos cálculos numéricos necessários à determinação da fronteira $\partial \Gamma$, primeiro nós vamos descrever os passos empregados para computarmos, isoladamente, as inúmeras soluções nas regiōes $\Gamma_{1}$ e $\Gamma_{2}$. Como já mencionamos, o que se segue é uma modificação do algoritmo empregado por $B \& W$ e cuja implementação será feita pelo uso de diferenças finitas.

Algoritmo II. Sejam, com $n \geq 1, x_{2_{n}}, \sigma_{n}$ e $a_{n}$ os respectivos valores iterados de $x_{2}, \sigma$ e $a(\psi)$ em $\Gamma_{2}$.

1. Prescrevem-se $x_{2}, \sigma_{0}(\Phi, \psi)$ e $a_{0}(\psi)$ em $\Gamma_{2}$, usando as funções tentativa como definidas na sec.5.2.2.

2. Resolve-se a equação ordinária

$$
\hat{\sigma}_{\boldsymbol{F}}=-\left(\mathrm{x}_{\dot{q}}^{2}+\mathrm{x}_{*}^{2} / \mathrm{a}^{2} \sigma_{\mathrm{n}-1}^{2}\right)
$$

para determinar $\hat{\sigma}(\Phi, \psi)$ com as condições iniciais adequadas a cada caso 
considerado ( ver eqs. (5.12b), (5.15b) e (5.19b)).

3. Atualiza-se $\sigma(\Phi, \psi)$ pela média ponderada

$$
\sigma_{n}=\omega_{\sigma} \sigma_{n-1}+\left(1-\omega_{\sigma}\right) \hat{\sigma}
$$

em que $\omega_{\sigma}$ é um parâmetro de sub-relaxação. A atualização é imediata ao cálculo de $\hat{\sigma}(\Phi, \psi)$ em cada ponto.

4. Repetem-se os passos $\underline{2}$ e $\underline{3}$ ponto a ponto até a convergência de $\sigma(\Phi, \psi)$.

5. Resolve-se a equação diferencial de segunda ordem para $\hat{\mathbf{x}}_{2}$

$$
\left(a(\psi) \sigma \hat{x}_{2}\right)_{\phi}+\left(\hat{x}_{2 \phi} / a(\psi) \sigma\right)_{\phi}=0
$$

6. Atualiza-se $x_{2}$ pela média

$$
x_{n}=\omega_{x} x_{n-1}+\left(1-\omega_{x}\right) \hat{x}
$$

onde $\omega_{x}$ è um parâmetro de sub-relaxação.

7. Repetem-se os passos $\underline{2}$ a $\underline{6}$ até a convergência de $x_{2}$.

8. Calcula-se e atualiza-se $a(\psi)$ através de

$$
\hat{a}=\left(\left.\left.x_{2}\right|_{\phi=1} \sigma\right|_{\phi=1}\right)^{-1}, \quad a_{n}=\omega_{a} a_{n-1}+\left(1-\omega_{a}\right) \hat{a},
$$

respectivamente, onde $\omega_{\mathrm{a}}$ é um coeficiente também de sub-relaxação.

10. Repetem-se os passos $\underline{2}$ a $\underline{8}$ até a convergência para $a(\psi)$.

11. Determina-se $y_{2}$ através da equação ordinária (sobre as características)

$$
y_{2_{\Phi}}=-x_{2} / a(\psi) \sigma
$$

com a condição inicial y $(1, \psi)-\psi$.

Ao nivel de concepção, este algoritmo se distingue do adotado por $B \& W$ em dois aspectos. (a) emprega para o cálculo de $\sigma(\Phi, \psi)$ o método de Gauss e Seidel [27] que em princípio é bem mais rápido do que o de Jacobi; (2) a atualização de $\sigma(\Phi, \psi)$ é feita antes mesmo de se processar o cálculo de $x(\Phi, \psi)$ que na prática é o mais dispendioso em tempo de computação, mesmo se realizado por um método específico como o de Stone. Com esta modificação nós acreditamos ter reduzido o 
número de iterações dispensáveis ao cálculo de $x(\Phi, \emptyset)$, que a cada vez agora toma um valor mais refinado de $\sigma(\Phi, \psi)$.

A nossa interpretação global deste algoritmo é a de um conjunto de operaçães repetidas, com as quais vai-se gradativamente ajustando uma solução tentativa às condições de contorno do problema. Assim, por exemplo, a repetição do passo $\underline{8}$ implica nas sucessivas relaxações do campo elétrico sobre o eletródio emissor, com consequentes mudanças na distribuição de carga espacial no interior do dominio. Evolutivamente o espectro do campo avança em direção a uma configuração "saturada" final, que é a solução do problema, dentro de uma tolerância pré-estabelecida.

Algoritmo III. Sejam $x_{1}$ e $b_{n}$ os n-ésimos valores iterados de $x_{1} e$ $b(\psi)$ em $\Gamma_{1}(n \geq 1)$.

1. Prescrevem-se $x_{10}$ e $b_{0}(\psi)$ em $\Gamma_{1}$ por meio das funções tentativa das eqs. (5.10) e (5.11).

2. Calcula-se $\hat{\mathbf{x}}_{1}$ pela expressão

$$
\left(\mathrm{b}(\psi) \hat{\mathrm{x}}_{1_{\phi}}\right)_{\phi}+\left(\hat{\mathrm{x}}_{1_{\psi}} / \mathrm{b}(\psi)\right)_{\phi}=0
$$

3. Atualiza-se $x_{1}$ pela mesma média

$$
x_{1 n}=\omega_{x} x_{1 n-1}+\left(1-\omega_{x}\right) \hat{x}_{1}
$$

4. Repetem-se os passos $\underline{2}$ e $\underline{3}$ a té a convergência para $x_{1}$.

5. Calcula-se e atualiza-se $b(\psi)$ por, respectivamente,

$$
\hat{b}=\left(\left.x_{1}\right|_{\infty=1}\right)^{-1}, \quad b_{n}=\omega_{b} b_{n-1}+\left(1-\omega_{b}\right) \hat{b}
$$

6. Repetem-se os passos $\underline{2}$ e $\underline{5}$ até a convergência de $b(\psi)$.

7. Determina-se $y_{1}$ através de

$$
y_{1_{\Phi}}=-x_{1} / b(\psi)
$$

com a condição inicial $y_{1}(1, \psi)=\psi$ e a final $y_{1}(1, \psi)=0$ 
Algoritmo IV. Seja $x_{n}$ o $n$-ésimo valor iterado de $x$ sobre $\partial \Gamma(n \geq 1)$.

1. Prescreve-se $x_{0}$ sobre $\partial \Gamma$ por meio da função tentativa da eq. (5.4).

2. Aplica-se o algoritmo III para calcular $x_{1}, y_{1}$ e $b(\psi)$ na região $\Gamma_{1}$.

3. Aplica-se o algoritmo Il para calcular $x_{2}, y_{2}, \sigma$ e a( $(\psi)$ na região $\Gamma_{2}$.

4. Calcula-se $\hat{\mathbf{x}}$ por meio da condição

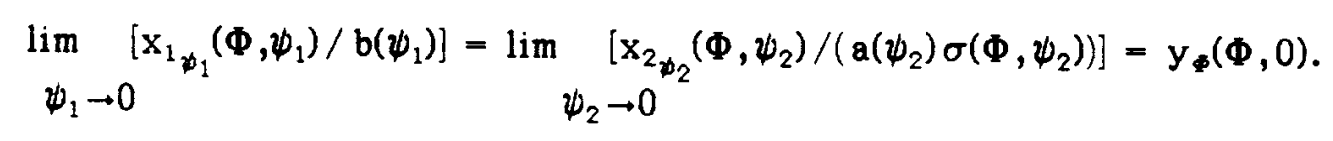

5. Atualiza-se $\mathrm{x}$ ainda pela média

$$
\mathrm{x}_{\mathrm{n}}=\omega_{\Gamma} \mathrm{x}_{\mathrm{n}-1}+\left(1-\omega_{\Gamma}\right) \hat{\mathrm{x}}
$$

em que $\omega_{\Gamma}$ é um parâmetro de sub-relaxação específico para $\mathrm{x}$ em $\partial \Gamma$.

6. Repetem-se os passos $\underline{2}$ a $\underline{5}$ até a convergência para $x$.

7. Determina-se y por meio de

$$
\left.\lim _{\psi_{1} \rightarrow 0}\left[y_{w_{1}}\left(\Phi, \psi_{1}\right) / \mathrm{b}\left(\psi_{1}\right)\right]=\lim \underset{\psi_{2} \rightarrow 0}{\left[y_{{ }_{\psi_{2}}}\right.}\left(\Phi, \psi_{2}\right) /\left(\mathrm{a}\left(\psi_{2}\right) \sigma\left(\Phi, \psi_{2}\right)\right)\right]=\mathrm{x}_{\boldsymbol{\Phi}}(\Phi, 0)
$$

Esta sucessão de passos equivale de fato permitir à fronteira $\partial \Gamma$ relaxar até que através dela, numa "última" configuração, se tenha satisfeito a condição de continuidade do campo elétrico.

Todos os cálculos descritos no Algorítmo IV são efetuados sobre os nodos do domínio discretizado da Fig.-5.2. As varreduras são feitas sistematicamente da esquerda para a direita e de baixo para cima. Em particular o cálculo da fronteira $\partial \Gamma$ está restrito à zona definida por $(M-3) \leq j \leq(M+3)$.

As equações compreendidas pelos Algoritmos II e III são aproximadas nos mesmos padrões descritos na sec.4.4 do capitulo anterior. Assim, para completarmos a formulação do nosso esquema mumérico resta apenas deduzir as equações de diferenças finitas para uma aproximação conveniente das condições de continuidade em (5.33) e (5.34). Considerando a molécula de discretização da Fig. - 5.3, nós podemos aproximar a eq. (5.33) pela seguinte identidade de diferença 
$+\psi_{0} 2 M$
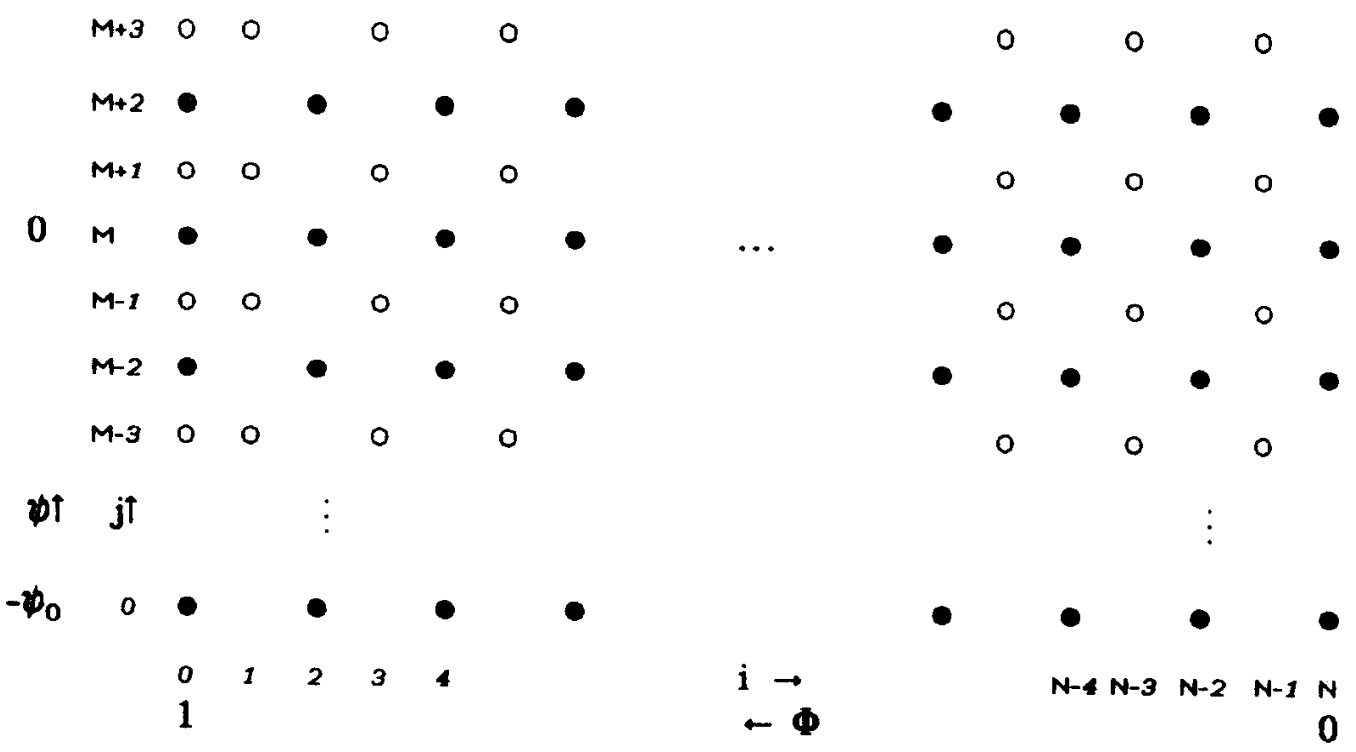

FIG. -5.2 Discretização do dominio da Fig. - 5.1(b). A linha nodal $j=M$ coincide com a fronteira $\partial \Gamma$. Todos os pontos nodais com $0 \leq \mathrm{j}<M$ estão em $\Gamma_{1}$, enquanto os demais em $\Gamma_{2}$. O espaçamento de grade e $h=|\Delta \Phi|=|\psi|$. $x(\Phi, \psi)$ e $y(\Phi, \psi)$ são aproximados nos nodos principais "๑" e as restantes funções nos secundários "o".

$$
\left[\frac{x(i, M)-x(i, M-2)}{h}\right] / b(M)=\left[\frac{x(i, M+2)-x(i, M)}{h}\right] /[a(M) \sigma(i, M)]
$$

O primeiro termo desta relação contém uma 'diferença para frente' enquanto o segundo uma 'diferença para trás'. Trata-se, portanto, de uma aproximação de primeira ordem $(\mathrm{O}(\mathrm{h})$ ).

\footnotetext{
$0(i-1, M+3) \quad O(i+1, M+3)$

- $(i, M+2)$

$\circ(i-1, M+1) \circ(i+1, M+1)$

- $(1, M)$

O $(1-1, M-1) \circ(1+1, M-1$

- $(i, M-2)$

O $(1-1, M-3) \quad 0(1+1, M-3)$
}

FIG.-5.3 Molécula de diferenca finita para aproximar $\mathrm{x}$ e $\mathrm{y}$ sobre $\partial \Gamma$. 
Não existindo nodos "o" sobre a linha $(i, M)$, o denominador $b(M)$ é aproximado por extrapolação linear dos correspondentes valores nas linhas $(i, M-1)$ e $(i, M-3)$, isto é,

$$
b(M)=\frac{3}{2} b(M-1)-\frac{1}{2} b(M-3)
$$

havendo uma expressão análoga para $a(M) \sigma(i, M)$, ou seja,

$$
\begin{gathered}
a(M) \sigma(i, M)=\frac{3}{2} a(M+1)\left[\sigma(i+1, M+1)+\sigma\left(i_{-1, M+1}\right)\right]- \\
-\frac{1}{4} a(M+3)\left[\sigma\left(i_{+1}, M+3\right)+\sigma\left(i_{-1}, M+3\right) .\right.
\end{gathered}
$$

Em termos destas definições para $b(M)$ e $a(M) \sigma(i-1, M+3)$, os valores de $x$ são calculados sobre $\partial \Gamma$ pela aproximação

$$
x(i, M)=\frac{[a(M) \sigma(i, M) x(i, M-2)-b(M) x(i, M+2)]}{[a(M) \sigma(i, M)+b(M)]} .
$$

Desenvolvendo um procedimento inteiramente análogo, demonstra-se facilmente que os valores de y em ar são aproximados de forma idêntica à de $x$ em (5.37).

No próximo capítulo nós comentaremos como na prática o Algoritmo IV convergiu em cada caso considerado. 


\section{CAPÍTULO VI}

\section{Resultados e Discussāo}

\section{1 - Introdução}

Neste capitulo apresentamos e discutimos os resultados da solução numérica do problema proposto no capitulo anterior, bem como aqueles relativos às duas experiências numéricas ali mencionadas.

Os resultados obtidos para as distribuições $x(\Phi, \psi)$ e $y(\Phi, \boldsymbol{\psi})$ são apresentados em conjunto na forma usual de reticulados llinhas de campo] $\times$ [curvas equipotenciais]. Nesses reticulados mantém-se uma rígida relação de escalas de modo a preservar as mesmas proporções de cada domínio considerado. Isto é necessário para que a noção de perpendicularidade entre os padrões de linhas de campo e equipotenciais seja transmitida corretamente.

Por uma questão de clareza nós apresentamos as distribuições calculadas de carga espacial em gráficos tridimensionais $\sigma(\Phi, \psi) \times x(\Phi, \psi) \times y(\Phi, \psi)$ ou $\rho(\Phi, \psi) \times x(\Phi, \psi) \times y(\Phi, \psi)$. Os valores de $x(\Phi, \psi)$ e $y(\Phi, \psi)$ são agora recalculados nos mesmos pontos nodais - "o" - que $\sigma(\Phi, \psi)$, por interpolação dos correspondentes valores calculados originalmente nos nodos "e" (ver Fig. -5.2 ou Fig. -4.2). O nosso propósito é mostrar, através desses gráficos, não só a evolução completa das distribuições de carga a partir das suas formas iniciais no eletródio emissor, mas também as possiveis flutuações que a propagação de erros pode introduzir no cálculo de $\sigma(\Phi, \psi)$. Certamente estes detalhes não poderiam ser revelados tão claramente em um gráfico de contorno, muito embora seja esta a forma usual de apresentação das distribuições $\sigma(\Phi, \psi)$.

Mostramos também os resultados obtidos para as distribuiçōes de campo elétrico sobre o eletródio emissor (ver eq. (4.17c)). A importância deste tipo de resultados torna-se evidente no fato de podermos compará-los diretamente com limites assintóticos esperados. Para exemplificar, consideremos o dominio da 
Fig. -5.1 (b). Na linha de campo $\psi=-\psi_{0}$, quando suficientemente afastada da região de carga espacial, a solução para o potencial elétrico é da forma $\Phi(x, y)=1-x$ (ver eq. (5.1d)). Portanto a distribuição de campo sobre o emissor deve tender para a unidade quando $\psi \rightarrow-\psi_{0}$. No extremo oposto, em $\psi=+\psi_{0}$, a solução é bem conhecida também e dada pela primeira das eqs. (5.1e). Obviamente, o limite calculado do campo sobre o emissor, quando $\psi \rightarrow+\psi_{0}$, deveria ser $E_{0}$ dado pela raiz real da última equação (5.1e), em que $\sigma_{0}$ é o valor conhecido do inverso da carga injetada também em $\psi=+\psi_{0}$.

Outros resultados igualmente significativos para o tipo de cálculo que processamos são aqueles relacionados com a condição de ortogonalidade entre linhas de campo e curvas equipotenciais, ou seja, $x_{\$} x_{b}+y_{6} y_{6}=0$. Invariavelmente para todos os casos estudados, calculamos uma aproximação para o primeiro membro desta identidade em cada ponto nodal "e" dos respectivos domínios. Infelizmente, por se tratar de tabelas numéricas volumosas, deixamos de apresentálos em toda a sua extensão. Indicaremos porém, em cada caso, o máximo desvio da condição e as regiões do reticulado onde ocorre com maior frequência.

Em todos os cálculos ef etuados consideramos um único espaçamento de grade $\underline{h=0.05}$. Para 'reproduzir' os resultado de B\&W nas condições das eqs. (5.15a -5.18), a que nos reportaremos doravante como CASO I, tomamos na grade da Fig. $-4.2 \psi_{0}=-5.0$ e $\psi_{1}=+5.0$ ou seja, cobrimos uma extensão de emissor equivalente a 10 distâncias $(l=1)$ entre os eletródios. Em outras palavras, consideramos uma rede de nodos " $\bullet$ " de $20 \times 200$ malhas $(N=40$ e $M=400)$. Uma rede semelhante de $20 \times 240$ malhas $\left(N=40, M=480, \psi_{0}=-6.0\right.$ e $\left.\psi_{1}=+6.0\right)$ foi utilizada para o cálculo nas condições das eqs. (5.19a - 5.22) - o CASO II neste capítulo. Lembre-se que neste último caso nós obtemos os dados de comparação da Fig. $-3.8(c)$ do cap. III.

Na determinação da fronteira $\partial \Gamma$, sob as diferentes condições de carga espacial definidas por $\rho_{0}=0.5,2.0,4.0,6.0\left(\sigma_{0}=1 / \rho_{0}\right.$ em $\left.(5.1 \mathrm{c})\right)$, nós empregamos uma grade idêntica à da Fig. $-5.2 \mathrm{com} \psi_{0}=4.0, N=40$ e $M=160$. Isto equivale a tomar uma rede de $20 \times 80$ malhas para cada uma das regiões $\partial \Gamma_{1}$ e $\partial \Gamma_{2}$. 


\section{2 - Sobre a Convergência dos Algoritmos}

Nesta secção nós descrevemos brevemente as condições em que os algoritmos II, III e IV (ver cap.V) serão ef etivamente convergentes.

Inicialmente fixamos em $\underline{12}$ o número máximo de iterações permissiveis a qualquer dos cálculos iterativos, significando dizer que além deste número o fluxo de processamento é interrompido. Em cada uma destas interrupções experimenta-se um novo conjunto de tolerâncias e parâmetros de relaxação. A experimentação numérica prossegue até que a convergência seja atingida. Daí em diante faz-se o que poderiamos chamar de ajuste fino de tolerâncias. Nesta fase, se uma maior precisão fosse requerida, seria razoável permitir um aumento conveniente no número máximo de repetições além do valor 12 prefixado.

$\mathrm{Na}$ prática estabelecemos os seguintes conjuntos de tolerâncias $(\delta)$ e parâmetros de relaxação:

TAB. I - Tolerâncias e parâmetros de relaxação.

\begin{tabular}{|c|c|c|c|c|c|}
\hline \multicolumn{2}{|l|}{ CASO I } & \multicolumn{2}{|l|}{$\underline{\text { CASO II }}$} & \multicolumn{2}{|c|}{ DETERMINACÃO DE $\partial \underline{\Gamma}$} \\
\hline $\begin{array}{l}\omega_{\sigma}=0.25, \\
\omega_{x}=0.50, \\
\omega_{\mathrm{a}}=0.25,\end{array}$ & $\begin{array}{l}\delta_{\sigma}=0.001 \\
\delta_{x}=0.015 \\
\delta_{\mathrm{a}}=0.020\end{array}$ & $\begin{array}{l}\omega_{\sigma}=0.25, \\
\omega_{x}=0.85, \\
\omega_{\mathrm{a}}=0.75,\end{array}$ & $\begin{array}{l}\delta_{\sigma}=0.005 \\
\delta_{x}=0.015 \\
\delta_{\mathrm{a}}=0.020\end{array}$ & $\begin{array}{c}\omega_{\Gamma}=0.25 \\
\omega_{\sigma}=0.25 \\
\omega_{x}=0.25 \\
\omega_{\mathrm{a}}=\omega_{\mathrm{b}}=0.25\end{array}$ & $\begin{array}{c}\delta_{\Gamma}=0.0005 \\
\delta_{a}=0.005 \\
\delta_{x}=0.015 \\
\delta_{a}=\delta_{b}=0.020\end{array}$ \\
\hline
\end{tabular}

Não podemos afirmar, entretanto, que estes conjuntos sejam os 'melhores' possiveis uma vez que o seu refinamento está fortemente limitado por tempos de processamento relativamente longos, como explicaremos a seguir.

A determinação de $x(\Phi, \psi)$ demanda, como já mencionamos, a resolução de um sistema de $((\mathrm{N}-2) / 2) \times((\mathrm{M}-2) / 2)$ equações lineares. Enquanto $\mathrm{B} \& \mathrm{~W}$ as resolveram pelo "Procedimento Fortemente Implicito" de Stone, que é um dos métodos mais rápidos e de aplicação especifica a problemas desta natureza [25],

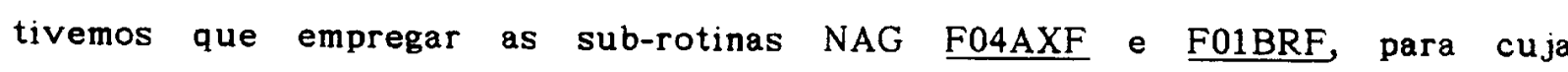


implementação sugerimos o modelo do Apêndice-A. Este método é talvez até mais preciso que o primeiro, porém quanto à taxa de convergência, não possuimos dados de comparação. Considerando o CASO l, em que o sistema linear consiste de 3781 equações, gasta-se cerca de $3.5 \mathrm{~min}$ de CPU em um computador VAX(vector) $6000-420$ para uma única iteração do cálculo de $x(\Phi, \psi)$. Neste exemplo, o laço interno do Algoritmo II (onde se calcula $x(\Phi, \psi)$ ) toma cinco iterações iniciais seguidas de uma depois, enquanto o laço externo (onde a( $\psi)$ é calculado) toma oito iterações, com um tempo total de 54min de CPU.

\section{3 -Resultados Numéricos do Caso I e Discussão}

Nas Fig. $-6.1(a),(b),(c),(d)$ temos, respectivamente, o reticulado de campo, a distribuição de carga espacial (inversa) e as intensidades do campo de injeção determinados numericamente para o CASO I.

No reticulado, as equipotenciais estão regularmente espaçadas por $\Delta \Phi=0.10$ e as linhas de campo por $\Delta \psi=0.25$. A condição de ortogonalidade calculada apresenta um desvio máximo da ordem de grandeza de $10^{-3}$ acentuadamente em duas estreitas regiōes. Uma, onde a ocorrência do desvio é mais frequente, está delimitada pelas linhas de campo $\psi=1.40$ e $\psi=2.00$. A outra se estende por duas ou três linhas nas vizinhanças de $\psi=2.80$. Se observarmos as Fig. $-6.1(b)$,(c),(d) não vamos encontrar qualquer comportamento excepcional nestas regiōes, nem na distribuição de carga e nem na do campo de injeção. Assim o máximo desvio da condição de ortogonalidade deve estar, neste caso, associado intrinsecamente a erros inerentes ao procedimento numérico

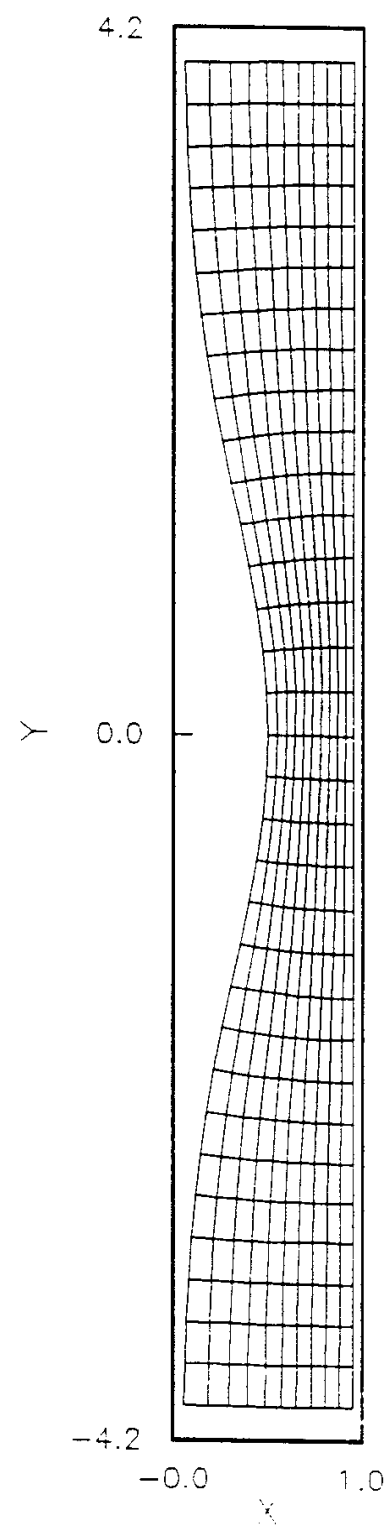

Fig. $-6.1(\mathrm{a})$ 
empregado, e não propriamente a valores grandes ou pequenos ou mesmo a mudancas bruscas da carga espacial ou do campo sobre o emissor. Pelos demais pontos do domínio o desvio está entre $10^{-4}$ e $10^{-7}$.

Considerando a magnitude das tolerāncias empregadas nos nossos cálculos, os dados da condição de ortogonalidade já constituem, por si só, um bom resultado, pois desvios mesmo da ordem de $3 \times 10^{-2}$ ou um pouco maiores seriam perfeitamente toleráveis.

É completa a semelhança entre o reticulado da Fig. $-6.1(a)$ e aquele da Fig.-4.2(i) do cap.IV, determinado por B\&W em idēnticas condiçōes de carga e geometria de eletródios (eqs. $(5.15 a, b, c)$ ). Muito embora a comparação seja de natureza qualitativa, é permitido afirmar, neste caso, que os nossos cálculos reproduzem os resultados encontrados por $B \& W$.

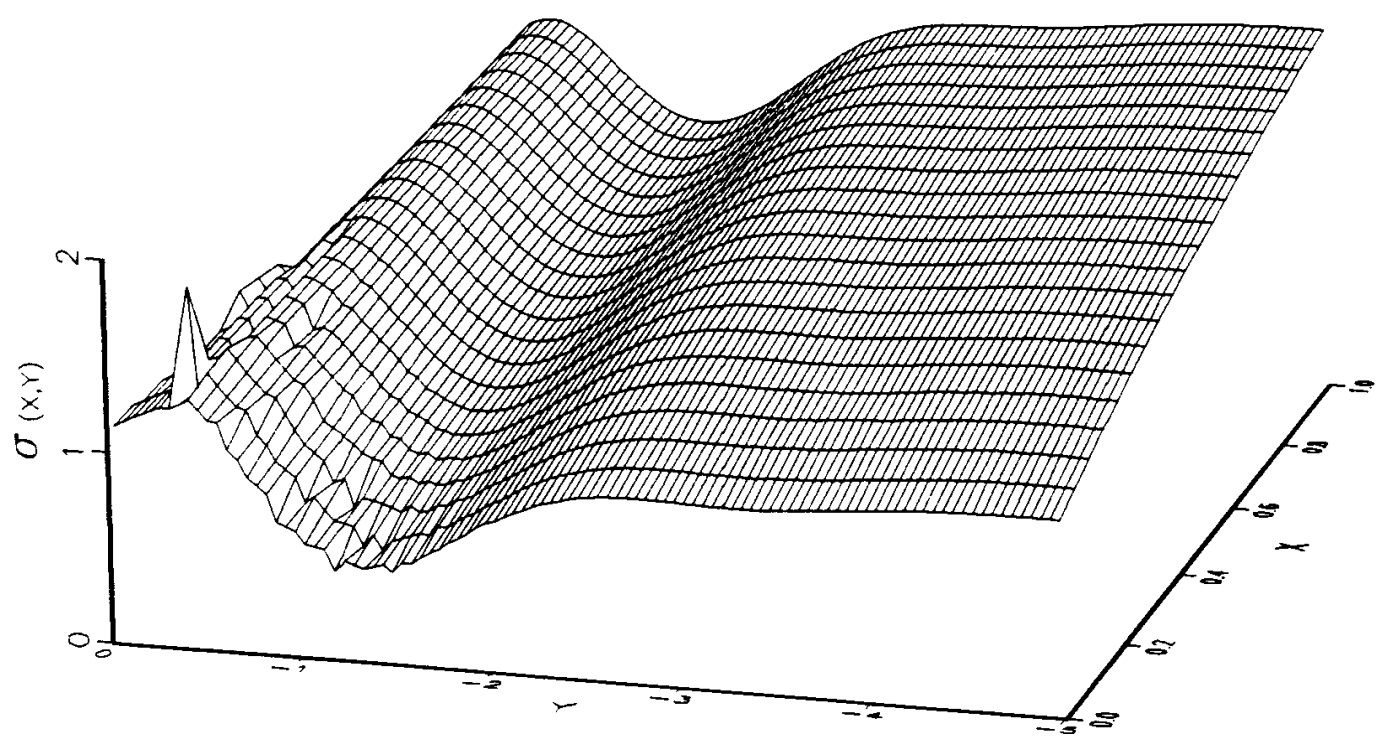

FIG. $-6.1(b)$

Nas Fig. -6.1(b),(c) observa-se comportamento esperado para a distribuição de carga no espaço inter-eletródios, ou seja, um decrescimento monotônico em direção ao coletor, o que se deve a um transporte mais rápido da 
carga em região de campo mais intenso. Por outro lado, é de alguma forma surpreendente que a distribuição de carga evolua espacialmente em direção ao outro eletródio, em $x=1$, preservando dominantemente a sua forma inicial no eletródio emissor em $x=0$.

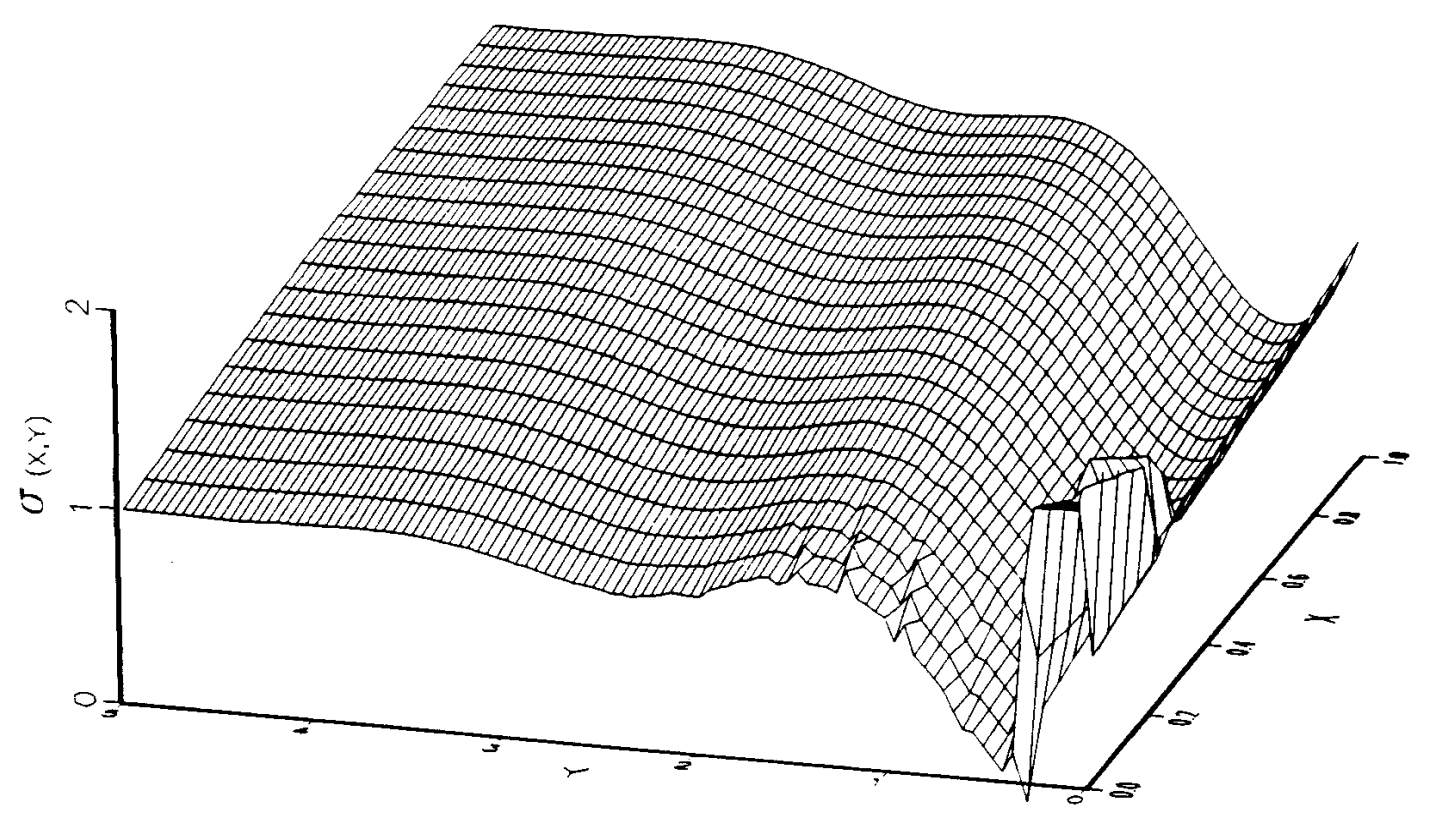

FIG. $-6.1(c)$

Isto é o que se observa mesmo ao longo das direções sobre as quais a carga apresenta um máximo elou um minimo locais. Tudo ocorre como se o fluxo de carga fosse dominado inteiramente pelo campo externo da estrutura de eletródios.

Da mesma forma, na Fig. $-6.1(a)$, não se observa nenhuma perturbação nas linhas de campo nas regiōes em que a carga possui valores relativamente maiores. Pelo exposto, conclui-se que possivelmente se trate ainda de um regime de pequena carga espacial onde a suposição de Deutsch torna-se satisfatória. Entretanto, somente novos cálculos em condicões de maior carga espacial e/ou de campos menos intensos nos permitiriam uma resposta definitiva. Voltaremos a este problema no cap. VII. 
A distribuiçao de campo no eletródio fonte, conforme a Fig.-6.1(d), é também da forma esperada.

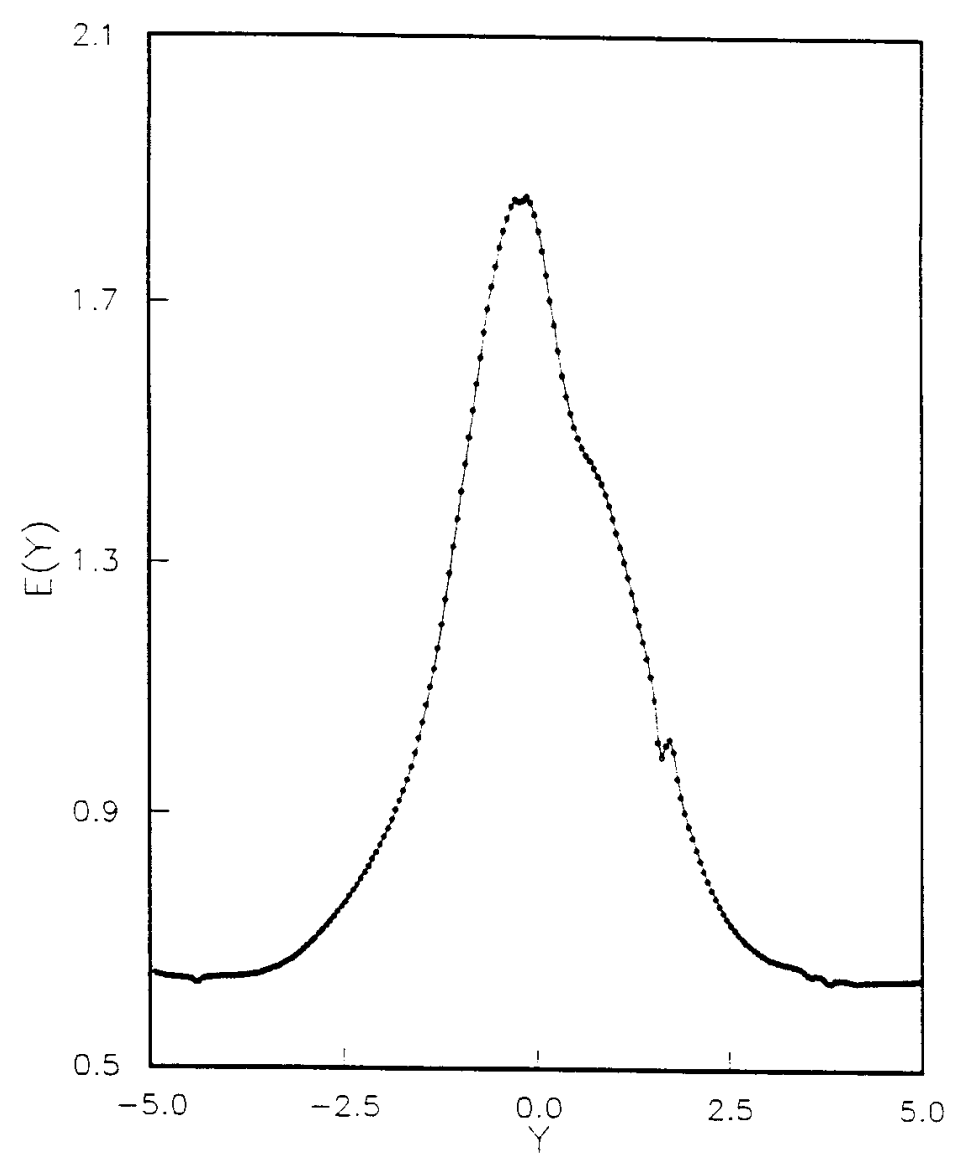

FIG. -6.1(d)

Por exemplo o pico principal observado na região de $\psi=0$ é devido a duas causas independentes. Por um lado ele é coincidente com a maior depressão da distribuição de carga como deve ser; por outro ele ocorre na região em que os eletródios estão nas vizinhanças da menor distância entre si. Além disto, os seus valores esperados e calculados nos limites são respectivamente, em $\psi=-5.0: 0.6345$ e 0.6514 ; e em $\psi=+5.0: 0.6324$ e 0.6393 .

Uma comparação mais quantitativa entre os nossos cálculos e os correspondentes de B\&W poderá ser tomada nos gráficos de contorno de $\sigma(\Phi, \psi)$ mostrados na Fig.-6.1(e),(f). Ali o gráfico (e) é o nosso resultado, sendo o outro, (f), uma cópia ampliada da Fig. -4.6(ii) do cap. IV (sec.4.4). A semelhança entre os 
resultados é bem visivel.

(e)

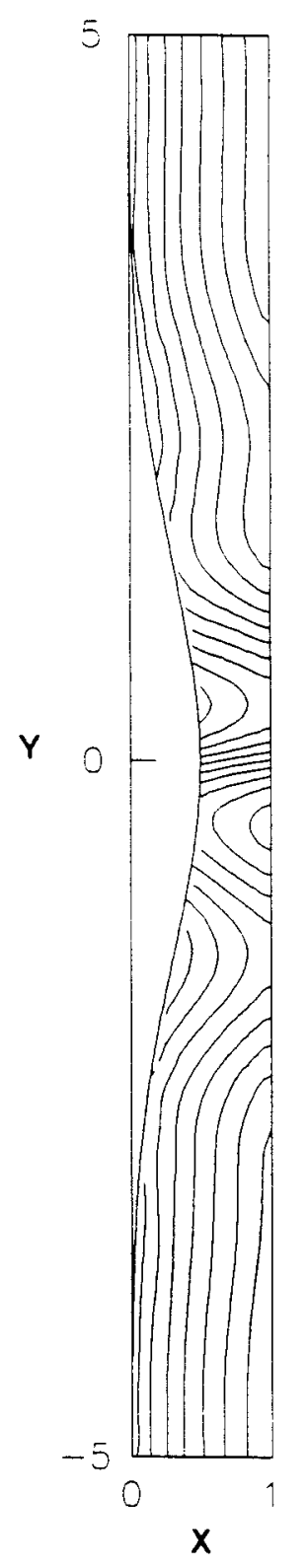

(f)

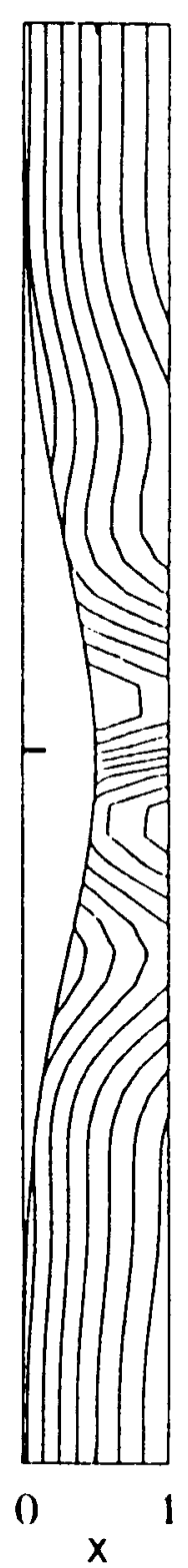

FIG. -6.1

\section{4 -Resultados Numéricos para a Linha de Corrente ar-Discussão}

As linhas de campo que emanam da origem em cada um dos reticulados das Fig.-6.2(a),(b),(c),(d) são fronteiras $\partial \Gamma$ (ou linhas de corrente extremas) calculadas numericamente em quatro distintas condições de carga espacial como 
explicaremos a seguir.

(a)

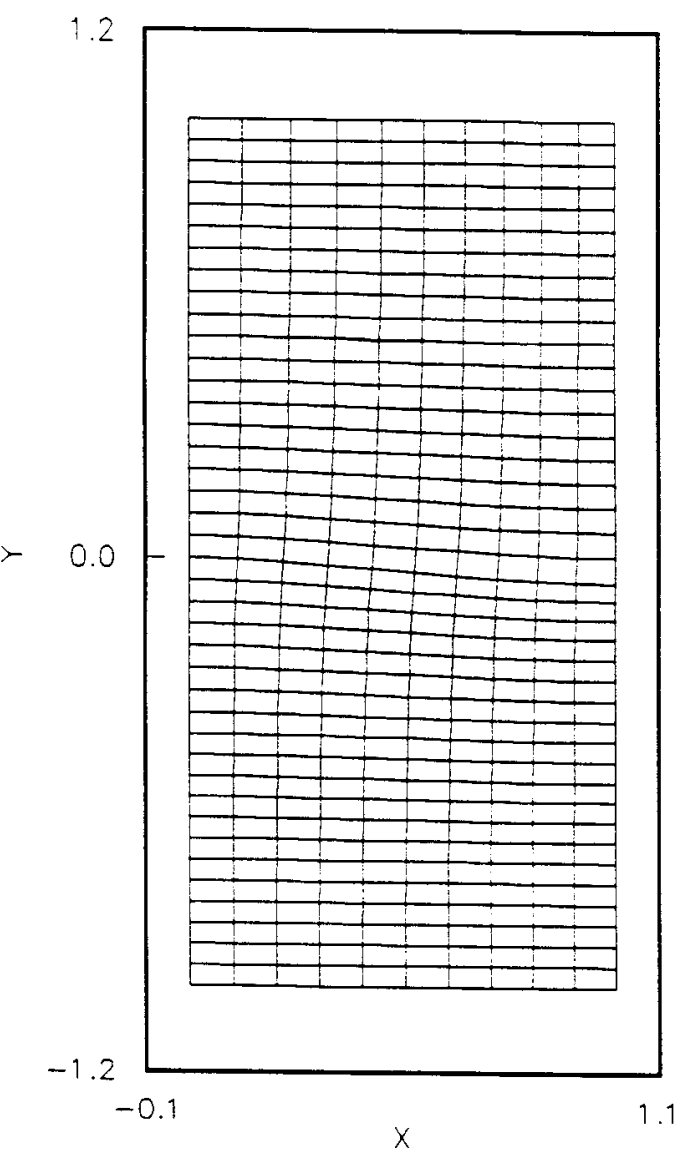

(c)

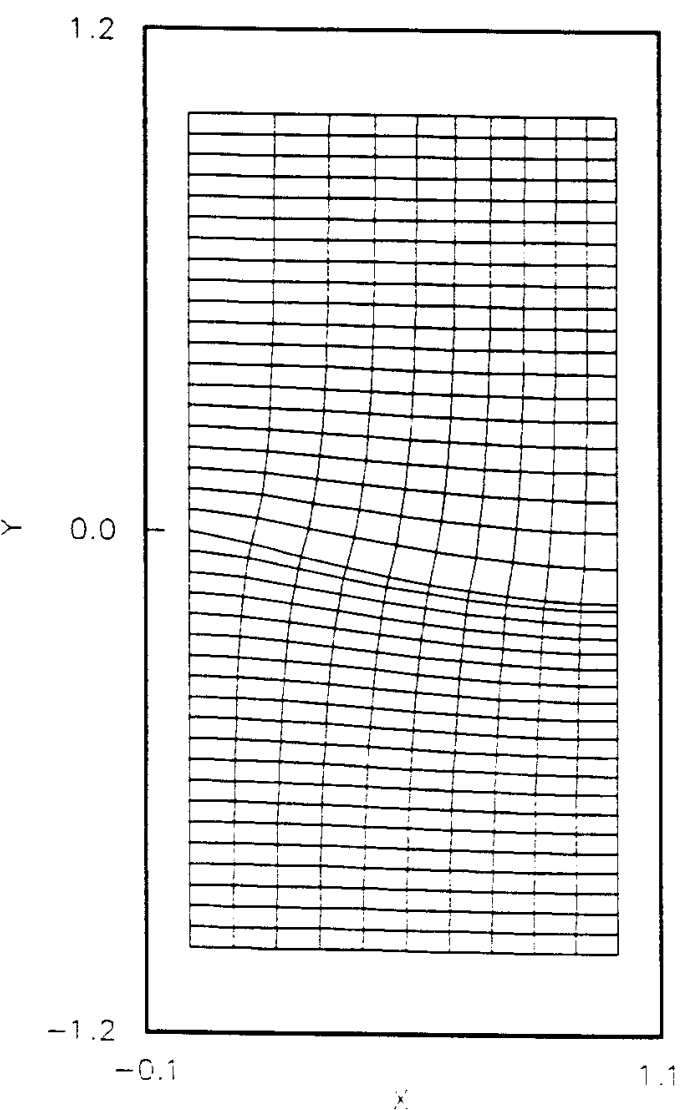

(b)

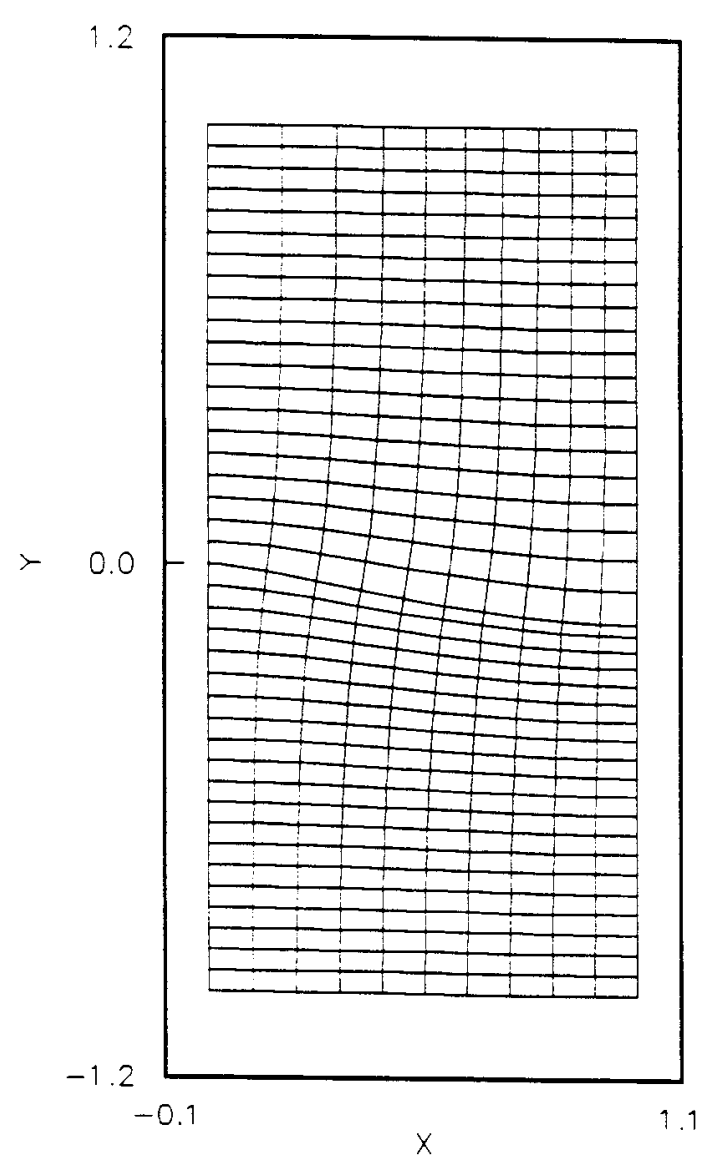

(d)

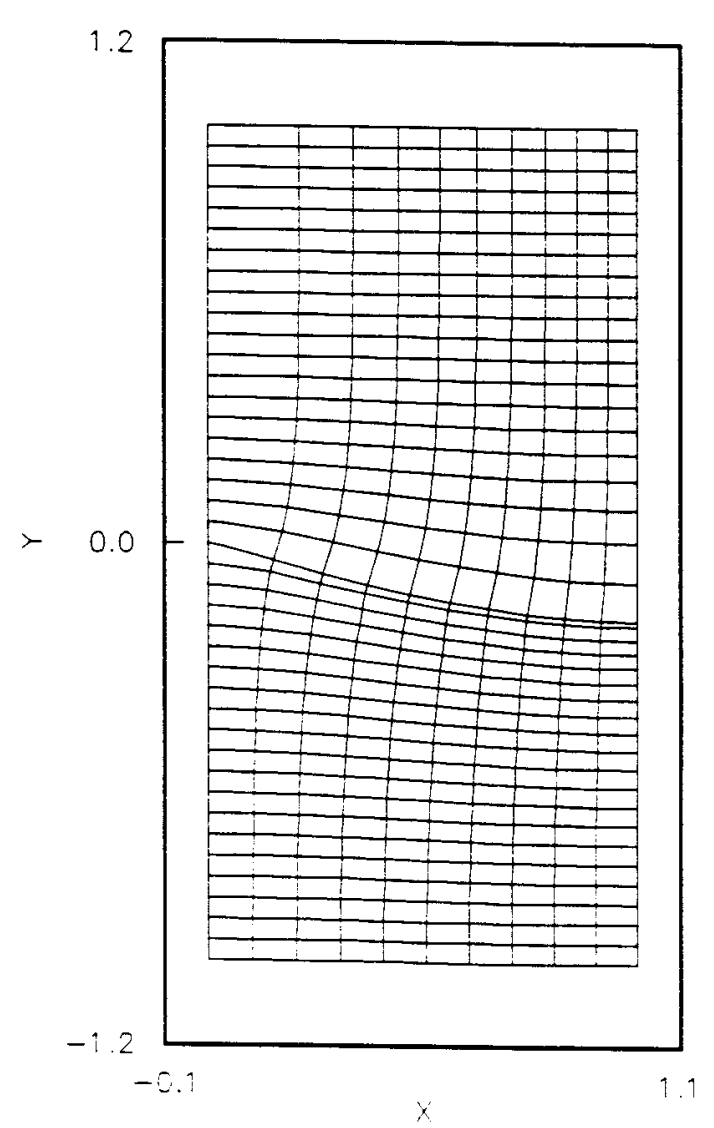

FIG. -6.2 
Da primeira para a última destas figuras, a carga injetada na região de Poisson - a porção superior dos reticulados onde o campo é menos uniforme tem os valores $\rho_{0}=0.5,2.0,4.0$ e 6.0. 0 espaçamento das linhas de campo é agora $\Delta q=0.05$, isto é, o próprio parâmetro da grade empregada nos cálculos ou ainda, um vigésimo da separação entre os eletródios. Por outro lado tem-se ainda $\Delta \Phi=0.1 \mathrm{em}$ todos os casos.

Relembremos aqui que de acordo com nossa parametrização (ver cap. IV), $y=\psi$ no eletródio emissor $(x=0)$ e isto explica porque as linhas saem igualmente espaçadas do mesmo. Por outro lado o avanço ao longo de uma linha de campo gera um valor aproximado para o campo elétrico cuja variação, ainda ao longo da mesma linha, daria um valor aproximado para a densidade de carga. De posse destes valores, o deslocamento ao longo das equipotenciais nos forneceria um valor aproximado para $a(\psi)$ e $b(\psi)$, já que na região de Poisson $a(\psi) d \psi=$ [incremento do fluxo de corrente] e na de Laplace $b(\psi)=[$ incremento do fluxo de campo elétrico].

Enquanto as Fig. -6.2 se destinam a mostrar a disposição relativa das curvas $\partial \Gamma$ na coletividade das demais linhas de campo (de carga espacial e laplacianas), na Fig. -6.3 nós mostramos estas curvas (วГ) em comparação entre si, para uma melhor compreensão dos efeitos do crescimento da carga sobre elas.

Na Fig. -6.7 apresentaremos a distribuição de carga espacial correspondente à situação em que $\rho_{0}=2.0 \mathrm{e}$, na Fig.-6.8, as distribuições de intensida-

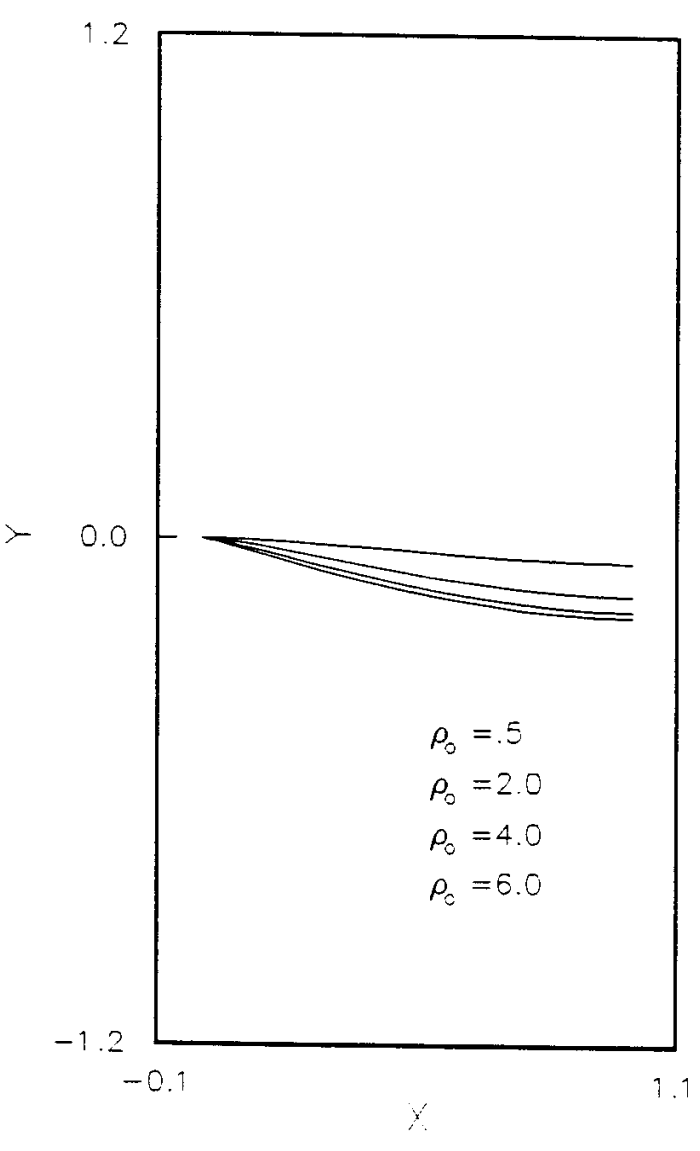
des de campo sobre o eletródio emissor.

FIG. -6.3 
Nas quatro situações estudadas, as fronteiras $\partial \Gamma$ (calculadas) esboçam os aspectos esperados, em particular, o encurvamento para baixo, mostrando o avanço da carga em direção à 'região de Laplace'. Na ordem das Fig.- 6.2, este avanço corresponde aproximadamente a 1, 3, 3.3 e 3.5 espaçamentos de grade $(h-0.05)$ em $x=1$, o que indica uma rápida tendência à saturação, como melhor se vê na Fig.-6.3. Cálculos adicionais foram feitos com $\rho_{0}=8.0$ e 10.0 para observarmos a progressão no encurvamento das fronteiras $\partial \Gamma$. Os resultados obtidos indicam, porém, que além de $\rho_{0}=6.0$ a situação não mais se modifica. Isto pode ser completamente explicado pelo fato de não se conseguir aumentar substancialmente a carga espacial entre os eletródios quando $\rho_{0}$ cresce além de 6.0 (a condição de d.d.p constante entre os eletródios limita o conteúdo da carga espacial entre estes).

A descontinuidade da carga injetada, por outro lado, produz uma perturbação mais ampla nos padrões de linhas de campo. Ela (a perturbação) se estende, em estimativa grosseira, no total de 1 a 1.5 da distância entre os eletródios quando a carga $\left(\rho_{0}\right)$ varia de 0.5 a 4.0 , porém mais profundamente na 'região de Laplace' do que na de Poisson.

O cálculo da condição de ortogonalidade produz resultados satisfatórios para os três menores valores da carga injetada. Na condição $\rho_{0}=0.5$, ou reticulado de Fig.-6.2(a), o desvio do zero é dominantemente menor do que $10^{-3}$. Sobre os pontos de $\partial \Gamma$ e da linha de corrente vizinha, o desvio é sistematicamente da ordem de $10^{-3}$. Porém em alguns poucos pontos isolados da região de Laplace, consideravelmente distantes da carga espacial, o desvio assume valores de $2 \times 10^{-2}$ a $2.5 \times 10^{-2}$.

No reticulado da Fig.-6.2(b), em que $\rho_{0}=2.0$, o desvio na ortogonalidade assume o valor máximo de $3.9 \times 10^{-2}$ nos pontos iniciais de $\partial \Gamma$, entre as duas primeiras equipotenciais, e cai para $10^{-3}$ nos pontos seguintes; persiste em $2 \times 10^{-2}$ no trecho inicial das quatro linhas de corrente imediatamente acima de $\partial r$.

Com o crescimento da carga aos valores $\rho_{0}=4.0$ e 6.0 , a ortogonalidade apresenta desvios cada vez maiores. Na Fig. $-6.2(\mathrm{c})$ isto se verifica entre as duas 
linhas de campo do topo da região de Laplace às dez seguintes na região de Poisson, notadamente na margem do eletródio emissor acima da fronteira $\partial \Gamma$. Aí os máximos calculados são 9,7 e $6 \%$. Em $\partial \Gamma$ propriamente, o desvio está abaixo dos 4\%. Em zona idêntica do reticulado da Fig. $-6.2(d)\left(\rho_{0}=6.0\right)$ calculam-se desvios de até $17 \%$, talvez porque, além da condição de carga $\rho_{0}=4.0$ as aproximações já não são mais adequadas.

O tempo de processamento dos cálculos cresce a $42 \mathrm{~min}$ com o crescimento da carga até $\rho_{0}=4.0$; daí decresce e cai abruptamente a partir de $\rho_{0}=8.0$, aos $6 \mathrm{~min}$ gastos quando $\rho_{0}=0.5$.

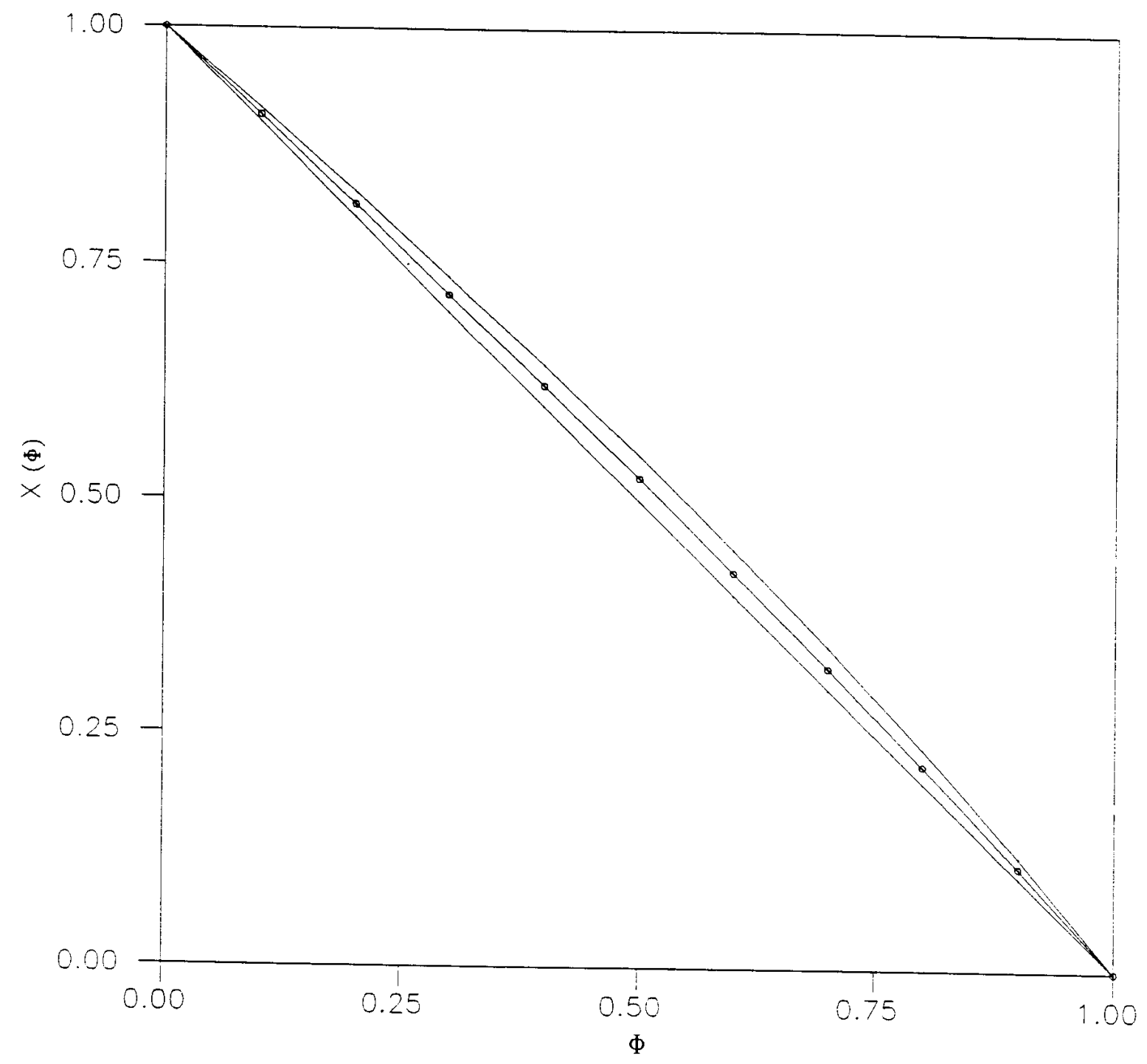

FIG. - 6.4 
As distribuições $x(\Phi, 0)$ calculadas sobre as linhas de corrente extremas (วГ) estão surpreendentemente em coincidencia com os valores fornecidos pela função tentativa, eq. (5.4) (sec.5.1, cap.V), que empregamos para dar início aos cálculos iterativos, seja qual for a condição de carga espacial considerada. Note-se aqui que $\circ$ número de iterações específicas para a obtenção da distribuição $x(\Phi, 0)$ é zero para $\rho_{0}=0.5$ e cresce para 4 e 6 quando $\rho_{0}=2.0$ e 4.0 . Neste último caso a distribuição calculada inicialmente se afasta para depois voltar à distribuição tentativa. Diante deste fato passamos a prescrever outras funçōes tentativa.

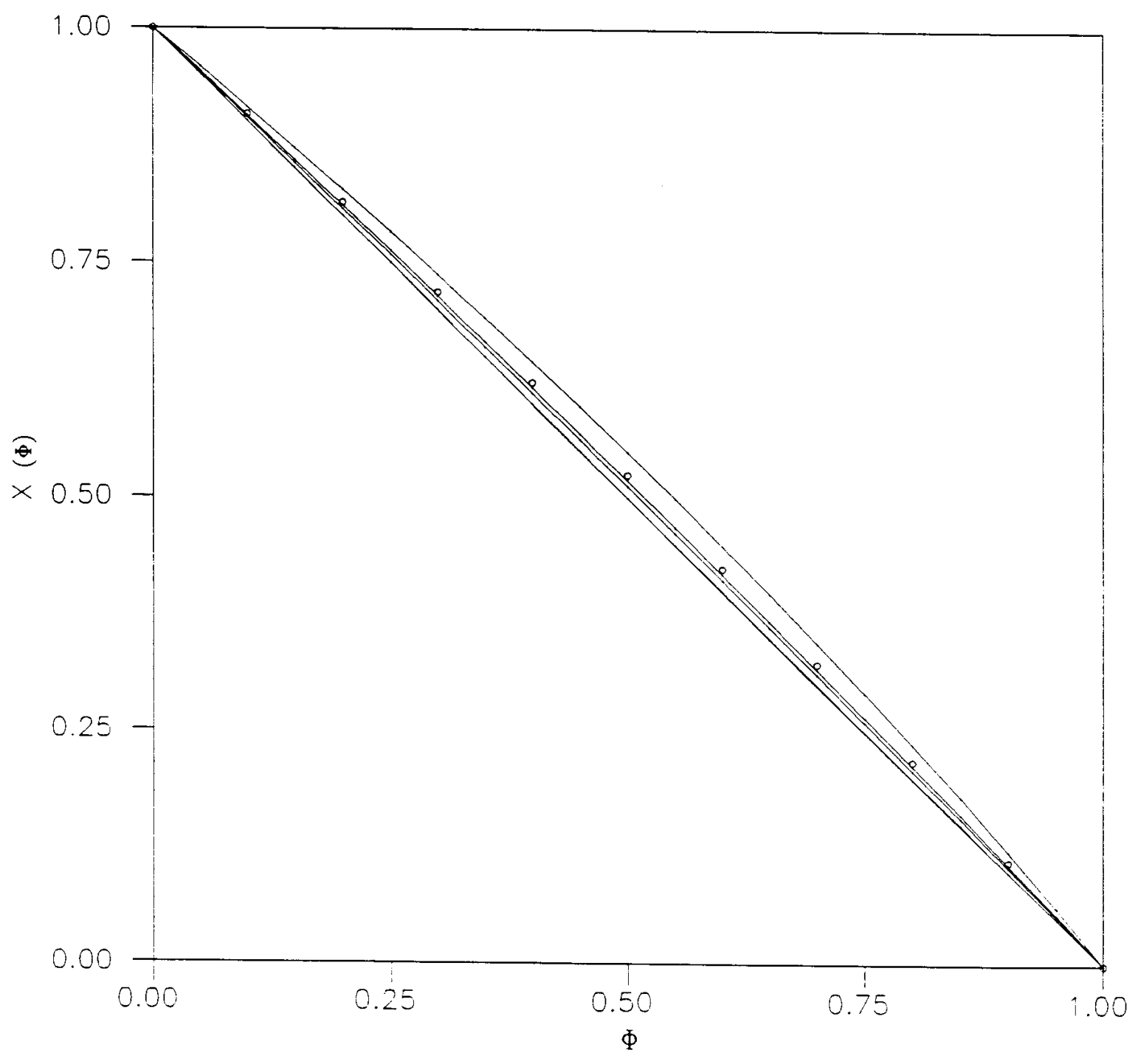

FIG. -6.5 
A intenção é verificar se nestas novas condições os cálculos ainda convergem à mesma solução $x(\Phi, 0)$ já obtida. Para melhor compreensão do que fizemos, consideremos reescrever a função tentativa da eq. (5.4) sob a forma

$$
\mathrm{x}_{(1)}(\Phi, 0)=\frac{1}{2}\{1-\Phi\}+\frac{1}{2}\left\{\frac{1}{2} \sigma_{0} \mathrm{E}_{0}\left[\left[1+\frac{3}{\sigma_{0} \mathrm{E}_{0}^{2}}(1-\Phi)\right]^{2 / 3}-1\right]\right\}
$$

A função do primeiro par de chaves desta equação é a reta da Fig.-6.4, enquanto a do segundo par é a curva superior; os circulos descrevem $\mathrm{x}_{(1)}(\Phi, 0)$ e a curva central com eles coincidindo é a solução a que convergiu o cálculo inicial.

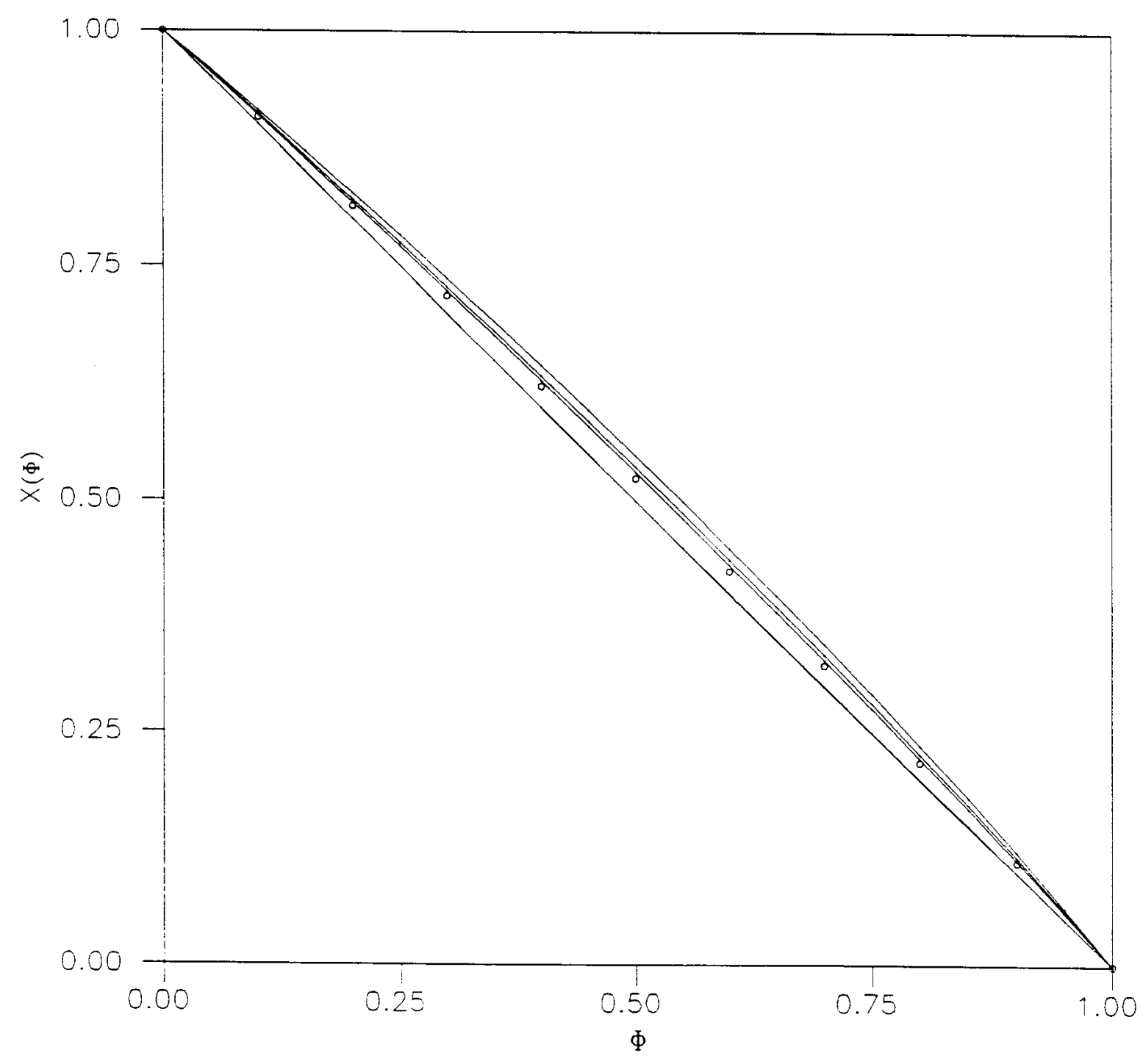

FIG. -6.6 
A situação de carga espacial é $\rho_{0}=0.5$. Um segundo cálculo é iniciado com a função tentativa

$$
\mathrm{x}_{(2)}(\Phi, 0)=\{1-\Phi\}
$$

e viu-se que não há convergência, pelo menos quando usamos o mesmo conjunto de parâmetros $\{\omega, \delta\}$ do cálculo precedente. No terceiro cálculo é tomada a função

$$
\mathbf{x}_{(3)}(\Phi, 0)=\frac{1}{2}\{1-\Phi\}+\frac{1}{2} \mathbf{x}_{(1)}(\Phi, 0)
$$

A convergência é alcançada se mudarmos $\omega_{x}$ de 0.25 para 0.5 (ver penúltima coluna da TAB.1, pp. 70) e os resultados estão mostrados na Fig. - 6.5: as curvas inferior e superior são como antes na Fig. - 6.4; a segunda, de baixo para cima, é $x_{(3)}(\Phi, 0)$, a terceira é a solução a que convergiu o cálculo enquanto os círculos são agora a solução calculada da Fig. -6.4 (ali em curva continua). Por último, no quarto cálculo, escolhemos a função tentativa

$$
\mathrm{x}_{(4)}(\Phi, 0)=\frac{1}{2}\left\{\frac{1}{2} \sigma_{0} \mathrm{E}_{0}\left[\left[1+\frac{3}{\sigma_{0} \mathrm{E}_{0}^{2}}(1-\Phi)\right]^{2 / 3}-1\right]\right\}+\frac{1}{2} \mathrm{x}_{(1)}(\Phi, 0) .
$$

Os parâmetros sendo ainda os mesmos do terceiro cálculo, verificamos que o tempo de processamento aqui é bem menor que o do caso precedente. Na Fig.-6.6 a segunda curva de cima para baixo é $\mathrm{x}_{(4)}(\Phi, 0)$, a terceira é a última solução na convergência e os círculos, outra vez, como na Fig. -6.5 .

A experimentação numérica descrita demonstra que o nosso Algoritmo IV não é suficientemente estável para nos permitir afirmar, conclusivamente, que a função tentativa da eq. (6.1) é a expressão analítica das distribuições $\mathrm{x}(\Phi, 0)$ sobre $\partial \Gamma$. Entretanto em um ponto é decisiva: - as soluções a que convergiram os últimos dois cálculos não são efetivamente as funções tentativa iniciais prescritas e elas se aproximam sistematicamente por ambos os lados da solução "esperada". Se variássemos outra vez $\omega_{\times}$de 0.5 para 0.25 nas últimas iterações do cálculo de $x(\Phi, 0)$, provavelmente melhor resultado seria obtido. Então é razoável e intuitivo admitir apenas que a função na eq. (6.1) é uma boa aproximação para as distribuições $\mathbf{x}(\Phi, 0)$. 
Os resultados encontrados para a distribuição da carga espacial na região de Poisson é de grande regularidade. Como se pode observar na Fig. -6.7, ela tem simetria planar até próximo à descontinuidade onde experimenta um crescimento suave indicando a presença de um campo elétrico menos intenso, como se pode inferir dos padrões mostrados nas Fig. - 6.2.

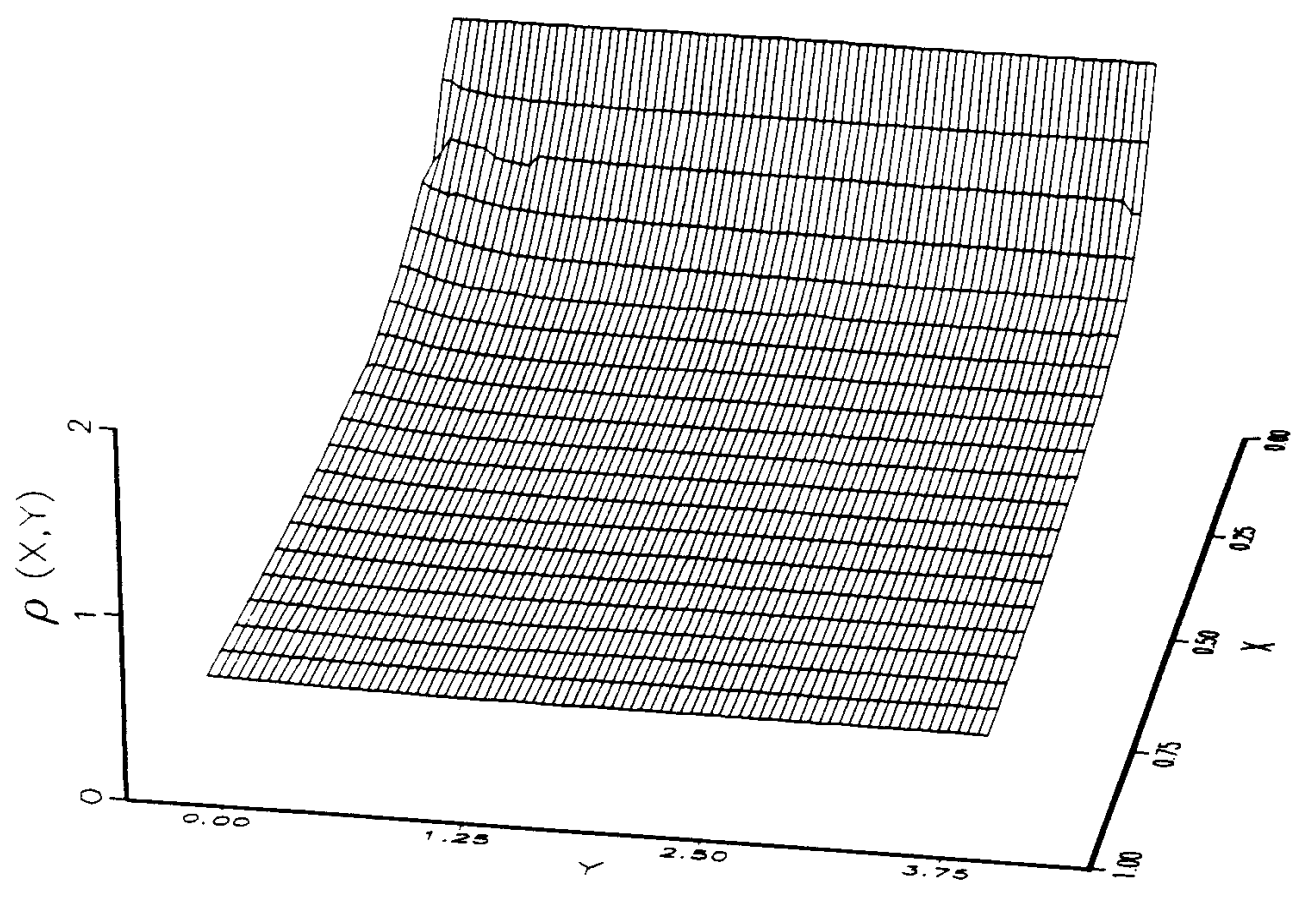

FIG. -6.7

Antes de comentar sobre os resultados da Fig.-6.8, é importante relembrarmos que a intensidade de campo elétrico pode ser calculada por uma das duas seguintes equações:

$$
\begin{gathered}
|E|=\left(x_{\psi}^{2}+y_{\phi}^{2}\right)^{-1 / 2} \\
|E|=\mathrm{a}(\psi) \sigma(\Phi, \psi)\left(x_{\psi}^{2}+y_{\psi}^{2}\right)^{-1 / 2}
\end{gathered}
$$

Quando a última é aplicada aos dois distintos segmentos do eletródio emissor (ver Fig. $-5.1(b))$, reduz-se às expressões muito simples 


$$
|E|=a(\psi) \sigma(1, \psi), \quad 0 \leq \psi \leq+\psi_{0}
$$

e

$$
|\mathrm{E}|=\mathrm{b}(\psi), \quad-\psi_{0} \leq \psi<0
$$

Foi com base nas eqs. (6.7) e (6.8) que realizamos o cálculo do campo, até porque, em princípio, são preferiveis à eq. (6.5) por não possuírem termos em derivadas.

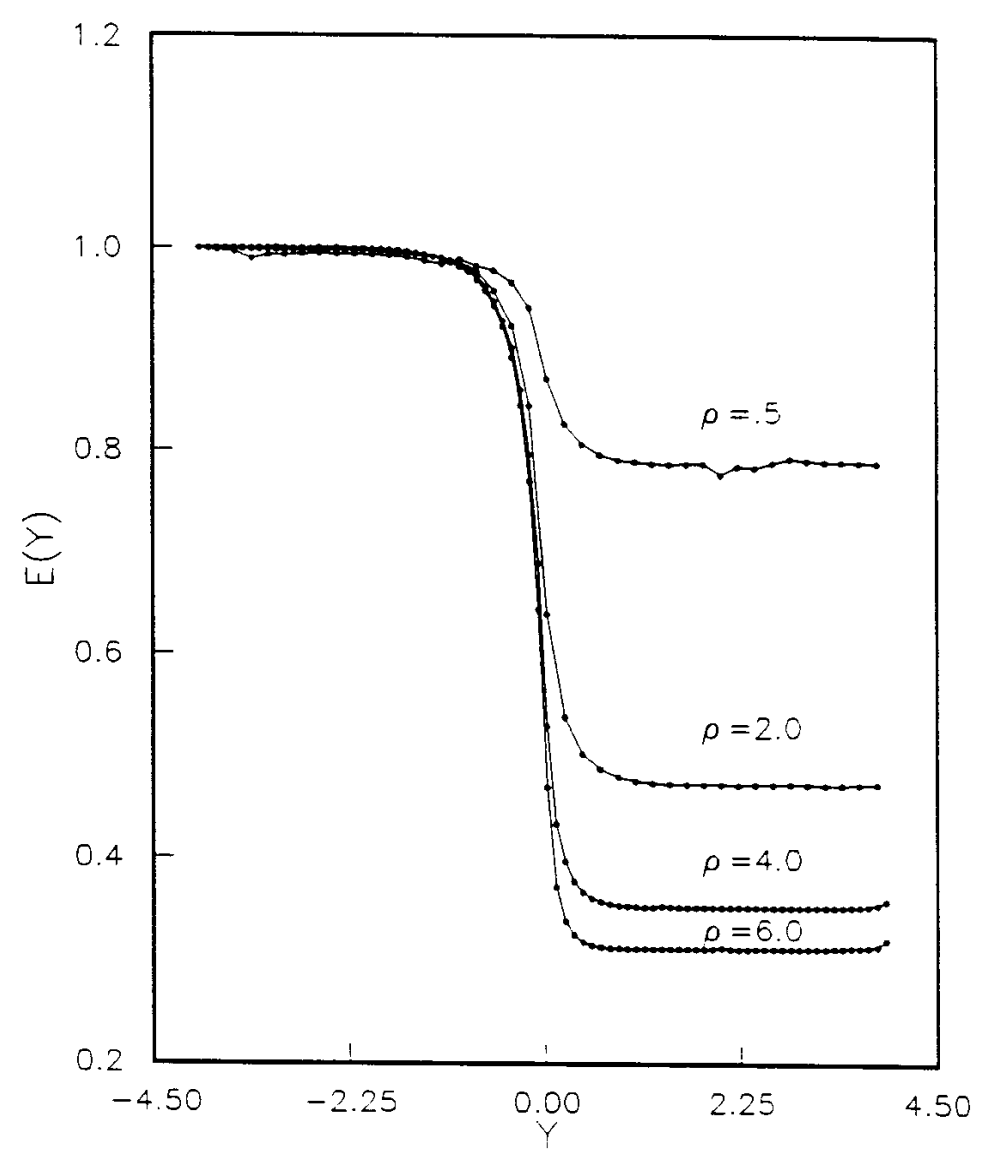

FIG. -6.8

As distribuições de campo para os diversos conteúdos de carga espacial tendem rapidamente à intensidade unitária e por esta razão estão em acordo com o que esperávamos na região não ativa do eletródio emissor. A TAB.II abaixo permite a comparação entre os valores esperados e os calculados no limite assintótico $\psi \rightarrow 4.0$, sobre o segmento propriamente emissor $(0 \leq \psi \leq 4.0)$. 
TAB. II - Valores esperados e calculados do campo elétrico sobre o emissor em $\psi \rightarrow 4.0$.

\begin{tabular}{|l|c|c|}
\hline \multirow{2}{*}{$\rho_{0}$} & \multicolumn{2}{|c|}{$E(4)$} \\
\cline { 2 - 3 } & V. esperado & V. calculado \\
\hline & & \\
0.5 & 0.7871 & 0.7885 \\
2.0 & 0.4385 & 0.4730 \\
4.0 & 0.2585 & 0.3583 \\
6.0 & 0.1800 & 0.3202 \\
\hline
\end{tabular}

Como podemos dá constatar, há uma diferença crescente entre estes valores à medida em que a carga espacial vai aumentando e supera os $10 \%$ após $\rho_{0}=4.0$.

Uma explicação plausivel para a discrepância acima, pode ser formulada a partir da eq. (6.7). Ora, nessa equação a intensidade de campo depende de uma única grandeza calculada iterativamente, que é a( () . Assim, se os valores do campo estão acima dos esperados, é porque a aproximação empregada para a( $\psi$ ) também fornece valores majorados.

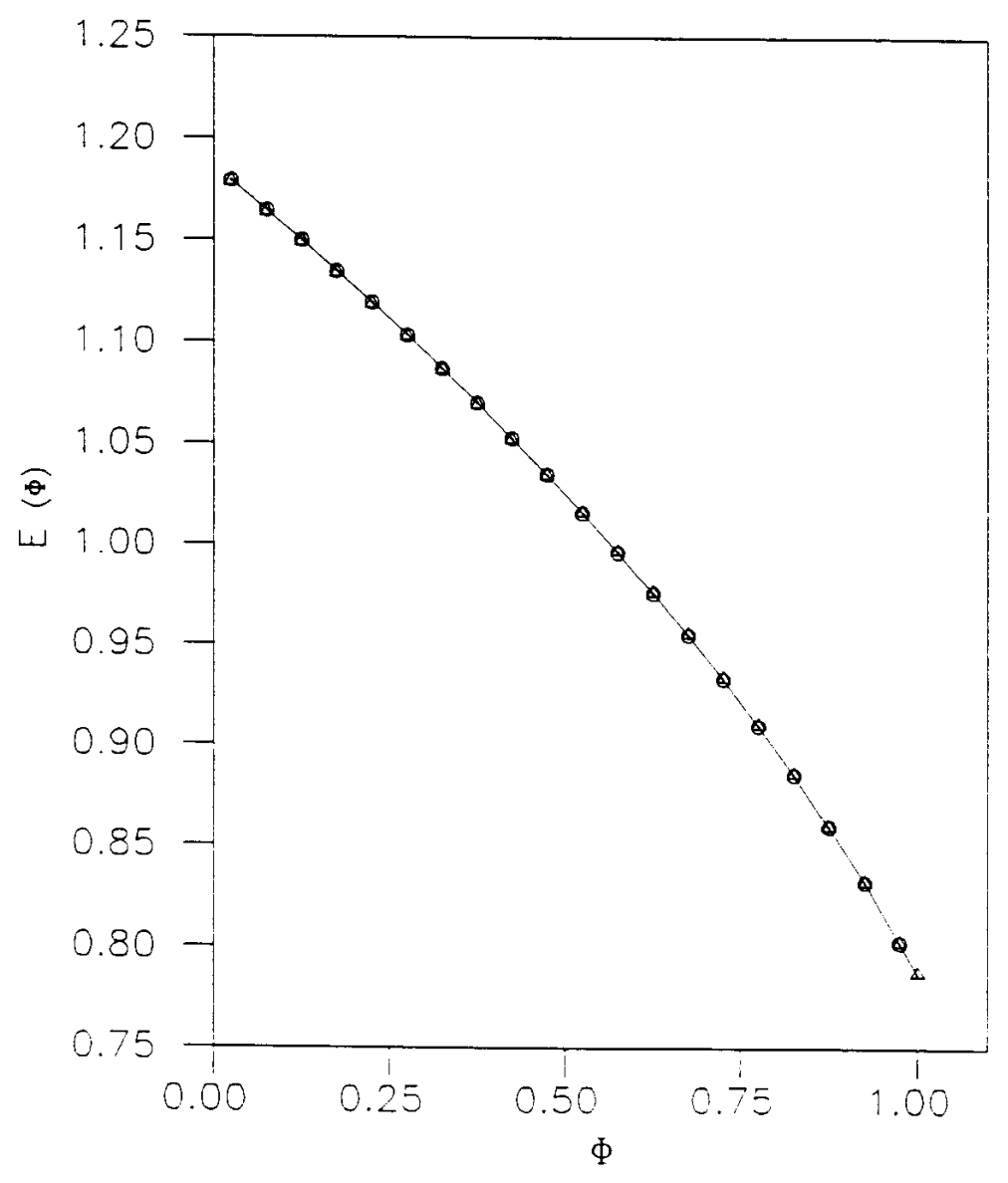

FIG. -6.9 
Para evidenciar melhor este fato, calculamos o campo elétrico ao longo da linha $\psi=3.825$, abaixo do limite $\psi=4.0$. Consideramos as situações de carga espacial $\rho_{0}=0.5,2.0$ e 4.0 cujos resultados estão mostrados nas Fig. - 6.9, 6.10, 6.11, respectivamente. As curvas continuas destas figuras são os valores obtidos pela solução exata - eq. (2.13b) do cap.II, os círculos são os valores calculados por uma aproximação de segunda ordem da eq. (6.5) enquanto os triângulos representam os valores de uma idêntica aproximação da eq. (6.6).

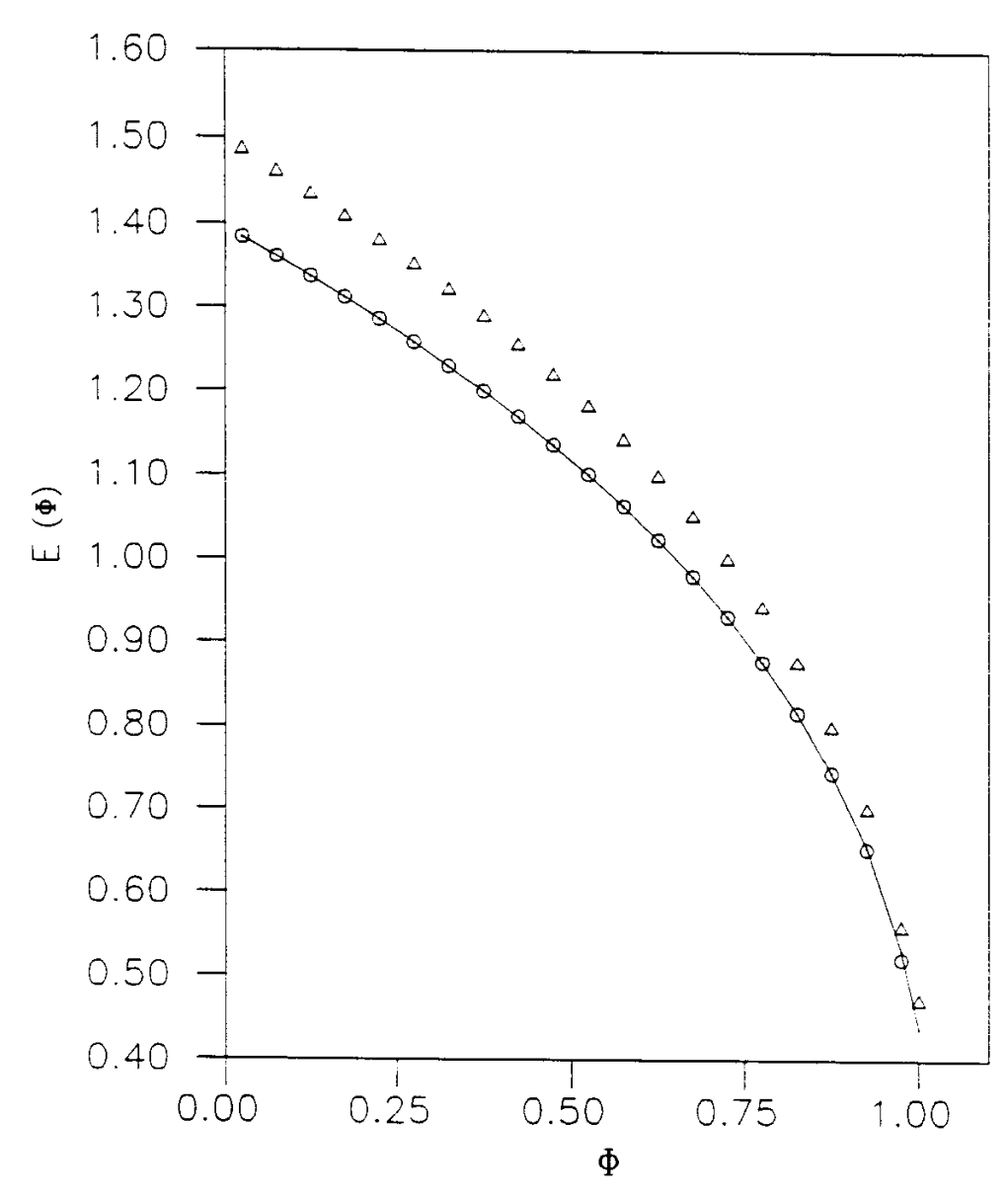

FIG. $-\mathbf{6 . 1 0}$

Para $\rho_{0}=0.5$ (Fig. - 6.9)) estes três diferentes cálculos são concordantes entre si. Porém, para $\rho_{0}=2.0$ e 4.0, somente os dois primeiros ainda fornecem os mesmos resultados, enquanto o terceiro, aquele que envolve a distribuição $a(\psi)$, resulta sempre em valores mais elevados, comprovando-se a nossa suposição. 


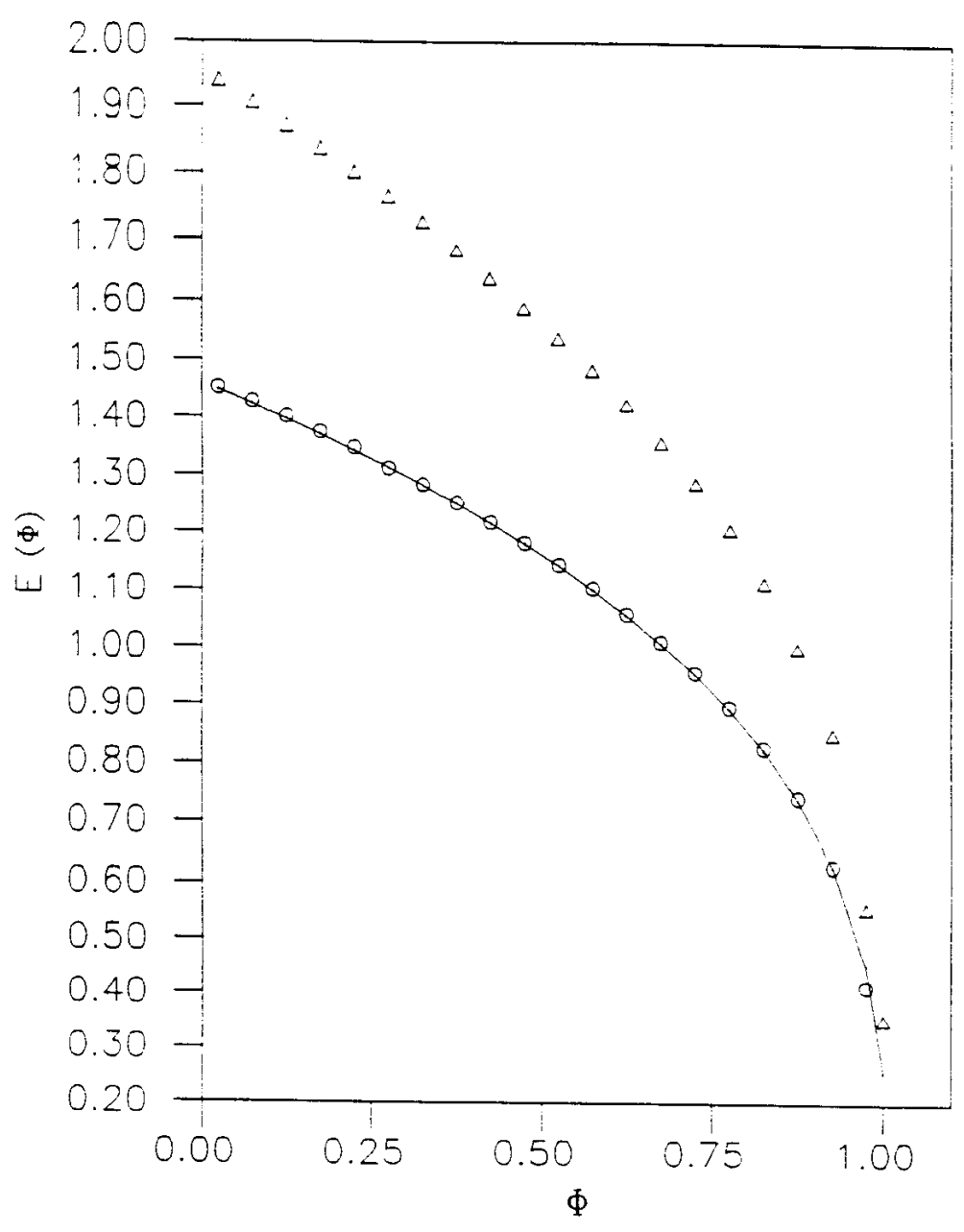

FIG. -6.11

A eq. (6.5) também não of erece uma boa aproximação para o campo sobre o eletródio emissor, pelo menos quando se envolve na sua discretização um número pequeno de pontos nodais. Por esta razão ela não foi usada para cálculo alternativo.

\section{5 -Resultados Numéricos do Caso II - Discussão}

Nesta secção nos limitaremos à apresentação de apenas três resultados típicos obtidos para o CASO II: o padrão de campo na Fig. -6.12 , os dados sobre a condição de ortogonalidade e os valores do campo de emissão, os quais representamos pela curva pontilhada da Fig. - 6.13. Nesta mesma figura a curva contínua dá o correspondente campo que tomados como condição de contorno no 
problema do Cap. III, isto é,

$$
E(y)=E_{0}\left\{1+\left(\frac{E_{0}^{\prime}-E_{0}}{2 E_{0}}\right)\left[1+\tanh \left(\frac{1}{2}(y-1)\right)\right]\right\}
$$

em que $E_{0} \simeq E(-6)=0.233$ e $E_{0}^{\prime} \simeq E(+6)=0.783$ são os valores esperados.

No reticulado (Fig. -6.12 ), as sete primeiras equipotenciais a partir de $\mathrm{x}=0$ estão espaçadas igualmente por $\Delta \Phi=0.15$ e as duas últimas por $\Delta \Phi=0.1$. As linhas de campo estão incrementadas de $\Delta \psi=0.25$.

Os desvios verificados na condição de ortogonalidade são da ordem de $10^{-3}$ ou menores para mais de 99\% dos pontos nodais internos. Todavia valores de $7 \%$ são observados precisamente nas linhas de campo quase limites, em $\psi=-5.950$ e 5.950 , não havendo uma explicação aparente para tal irregularidade.

Outra vez voltamos a enfatizar que é satisfatória a concordância entre as equipotenciais determinadas pelos dois diferentes tratamentos numéricos descritos neste trabalho, como se pode rever na Fig.-3.8(c), cap.lll. Apesar disto, a já mencionada falta de precisão no cálculo do campo sobre o eletródio emiossor, via eq. (6.7), torna completamente dispensável que igual concordância deva existir entre as curvas da Fig.-6.13. Mesmo assim, há um comportamento muito semelhante entre estes resultados: verifica-se que a curva pontilhada é bem aproximada pela eq. (6.9) quando nesta $E_{0}$ e $E_{g}^{\prime}$ são os valores extremos calculados 0.369 e 0.698 respectivamente.

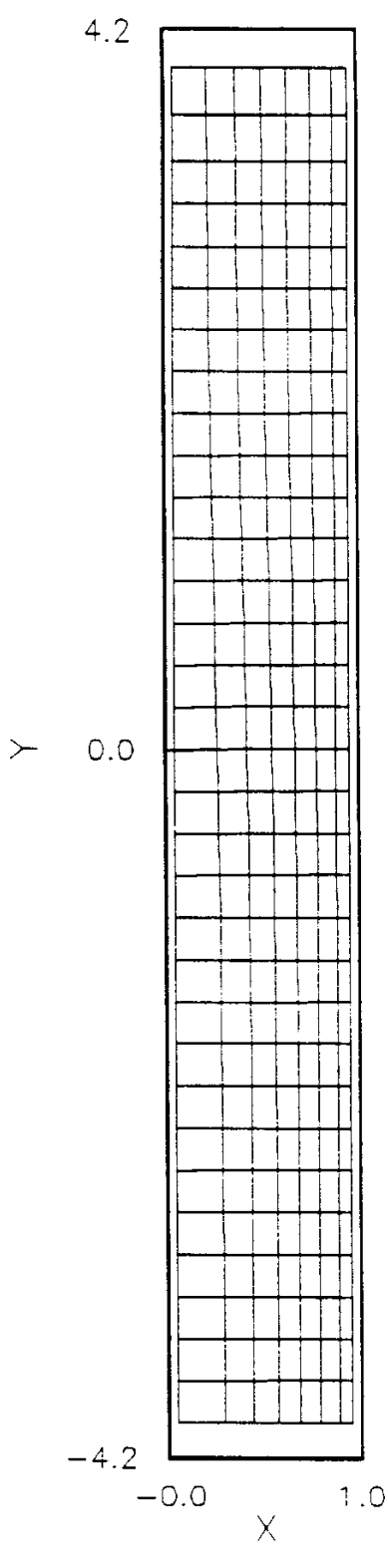

FIG. -6.12

Por último, ainda com relação aos dados da Fig. - 6.13, é necessário dizer que na região do emissor onde a carga é cerca de 0.5 , próximo a $y=6.0$, o valor calculado do campo é bastante diferente do esperado 
(0.783), ao contrário do que se vinha observando regularmente em todos os casos já estudados.

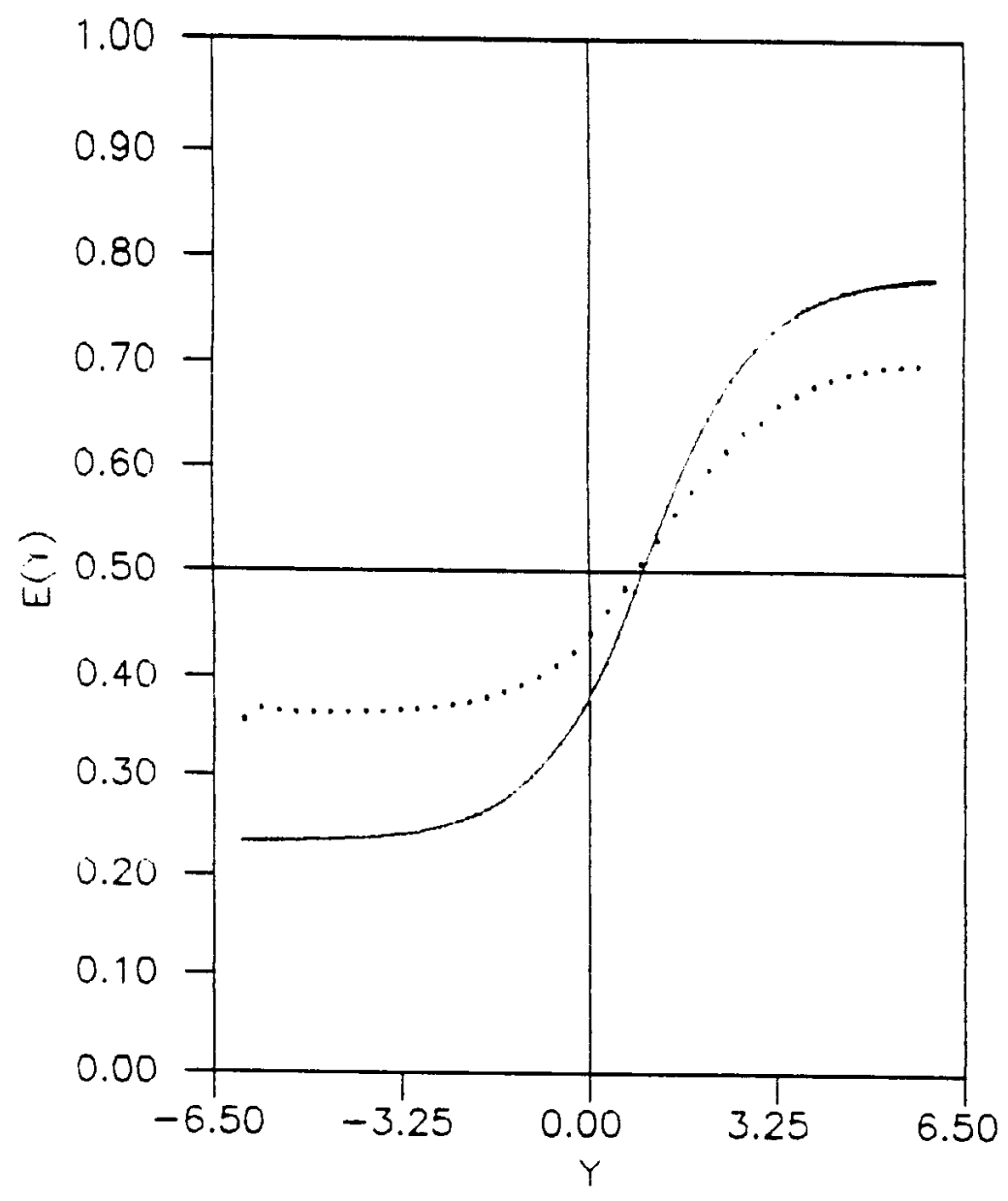

FIG. -6.13

\section{6 - Consideraçōes Finais}

Devemos informar, inicialmente, que além dos escassos resultados publicados por B\&W, o nosso elemento de comparação, quando possivel, tem sido a solução unidimensional do problema de carga espacial em geometria planar.

Com relacão aos primeiros, há fortes evidências de termos chegado a resultados semelhantes no que diz respeito ao cálculo de algumas grandezas de campo e isto nos encora.ja ao estudo das outras situações. Quanto a parâmetros de relaxação, tolerâncias e tempos de processamento não podemos traçar qualquer paralelo porque $B \& W$ não dão informações precisas sobre estes elementos. Também 
não temos qualquer informação sobre que funções tentativa teriam usado ou se a convergência do seu algoritmo (deles) teria alguma dependência marcante ou não com essas funções. Enfim, B\&W se ativeram muito pouco ao estudo de domínios simplesmente conexos.

Os nossos cálculos, a concluir de algumas comparações com a solução unidimensional exata, direta ou em limites assintóticos, têm se mostrado mais eficazes em condições de pequena carga espacial. Nas situações de maior carga a convergência se torna mais delicada e os resultados passam a ter maior dependência com as funções tentativa empregadas para as grandezas calculadas iterativamente. Por outro lado, dada a natureza global do cálculo, a se traduzir pelos sistemas lineares para a distribuição $\mathrm{x}(\Phi, \psi)$, podemos garantir uma certa estabilidade aos resultados embora discrepâncias localizadas sejam observadas. 


\section{A PÍTULO VII}

\section{A Suposição de Deutsch em Geometrias Simplesmente Conexas}

Enquanto analisávamos os resultados apresentados na sec.6.3 do capitulo anterior, não constatamos qualquer ação visivel da carga espacial sobre a direção das linhas do campo em que era transportada. De fato o padrão da Fig.-6.1(a) somente é identificável com um campo de carga espacial pela distribuição não uniforme das equipotenciais nos limites onde os eletródios então considerados tornam-se paralelos. O mesmo ocorre dentro da precisão visual com os padrões de campo obtidos no estudo do cap.III e depois repetido no cap.VI, Fig. -6.12 (sec.6.5).

Para evidenciar mais diretamente estas observações, nós vamos reapresentar o espectro de campo da Fig. -6.1(a) ao lado do correspondente para o campo harmônico (laplaciano) do mesmo sistema de eletródios, Fig.-7.1(b). Vê-se então que a presença da carga no espaço inter-eletródios tão somente modifica a distribuição das equipotenciais ao passo que as linhas de campo permanecem inalteradas. Não há dúvida, pois, de que estamos aqui - como nos casos citados acima - diante de uma situação onde a conhecida suposição de Deutsch tem ef etiva aplicabilidade.

Se por um lado a independência das linhas de campo com a carga espacial pode ensejar opção diferente de cálculo para o problema, talvez à guisa dos trabalhos pioneiros referidos no cap.II, por outro não pode naturalmente ser tomada como a regra geral, devendo se limitar estritamente a certas condições particulares em que se tem "baixos" conteúdos de carga fluindo em campos relativamente "elevados" impostos pela geometria de eletródios. Por esta razão seria interessante mostrar pelo menos um caso simples, próximo aos já estudados, onde a carga espacial distorce as linhas de campo em relação às laplacianas do mesmo sistema de eletródios. No que segue falaremos brevemente de duas 
experimentações numéricas realizadas com este objetivo.

(a)

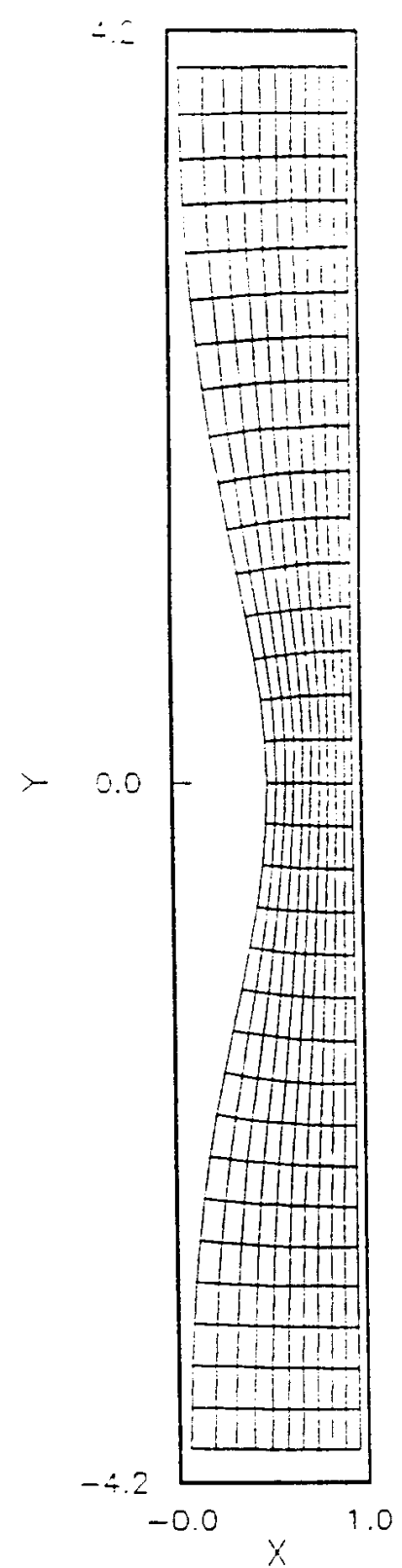

(b)

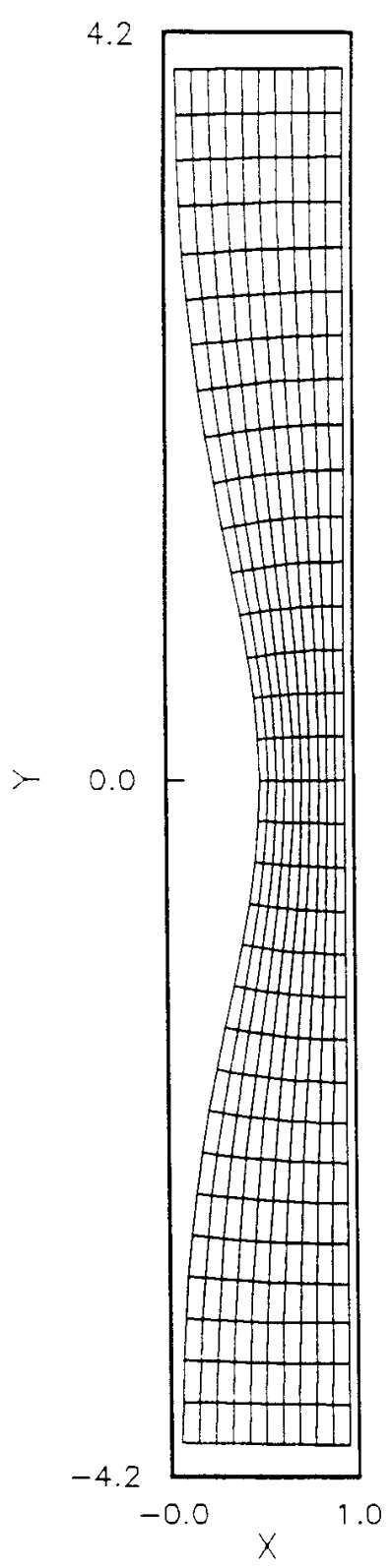

FIG. - 7.1

Inicialmente consideramos o sistema de eletródios plano-plano com deformacão gaussiana centrada em $\underline{y=0}$ e as densidades de carga (inversas) no emissor dadas por

$$
\sigma(1, \psi)=0.5-0.3 \exp \left(-0.5(\psi \pm 1)^{2}\right)
$$

De acordo com a eq. (7.1), tem-se sobre o eletródio ativo um patamar de densidade de magnitude igual a 2.0 , sobre o qual se superpõe um pico em $\psi= \pm 1$ (ou $y= \pm 1$ ) 
de valor igual a 5.0. Os resultados calculados ainda não revelaram 0 efeito esperado da distorção das linhas de campo nas proximidades de $y= \pm 1$; talvez porque os picos da carga tenham sido colocados ainda muito próximos do centro do emissor $(y=0)$, onde o campo é muito intenso. Apesar disto, um efeito interessante foi observado na distribuição da carga: o pico de carga se desloca para o coletor buscando a direção que virtualmente leva ao centro do emissor imagem refletido no plano coletor.

Finalmente retomamos os cálculos no sistema de dois eletródios planos infinitos, onde a contribuição de campo externo é bem mais regular, e estipulamos como condição de contorno a densidade de carga da forma

$$
\sigma(1, \psi)=1-1.1988 \exp (-|\psi|) \sin (3 \psi)
$$

Esta densidade tem um extremo de carga mais pronunciado do que o usual dado na eq. (5.15b) (sec.5.3.2, cap.V), o qual atinge o valor 4.0 nas proximidades de $y=\psi=0.4163$

Nas Fig. - 7.2(a),(b),(c) estamos mostrando em escala adequada diferentes porções do espectro de campo calculado numericamente. A ação da carga espacial prontamente se manifesta sobre as linhas de campo com origem nas vizinhanças de $y=\psi=0.4163$ e é um fenômeno grandemente localizado, como já haviamos observado em condições diferentes nas Fig.-6.2. Além desta visivel repulsão entre as linhas de campo, o padrão também esboça um comportamento oscilatório imposto pela densidade de carga, indicando que a disposição geométrica das linhas necessariamente é diferente do que seria no caso puramente laplaciano. 


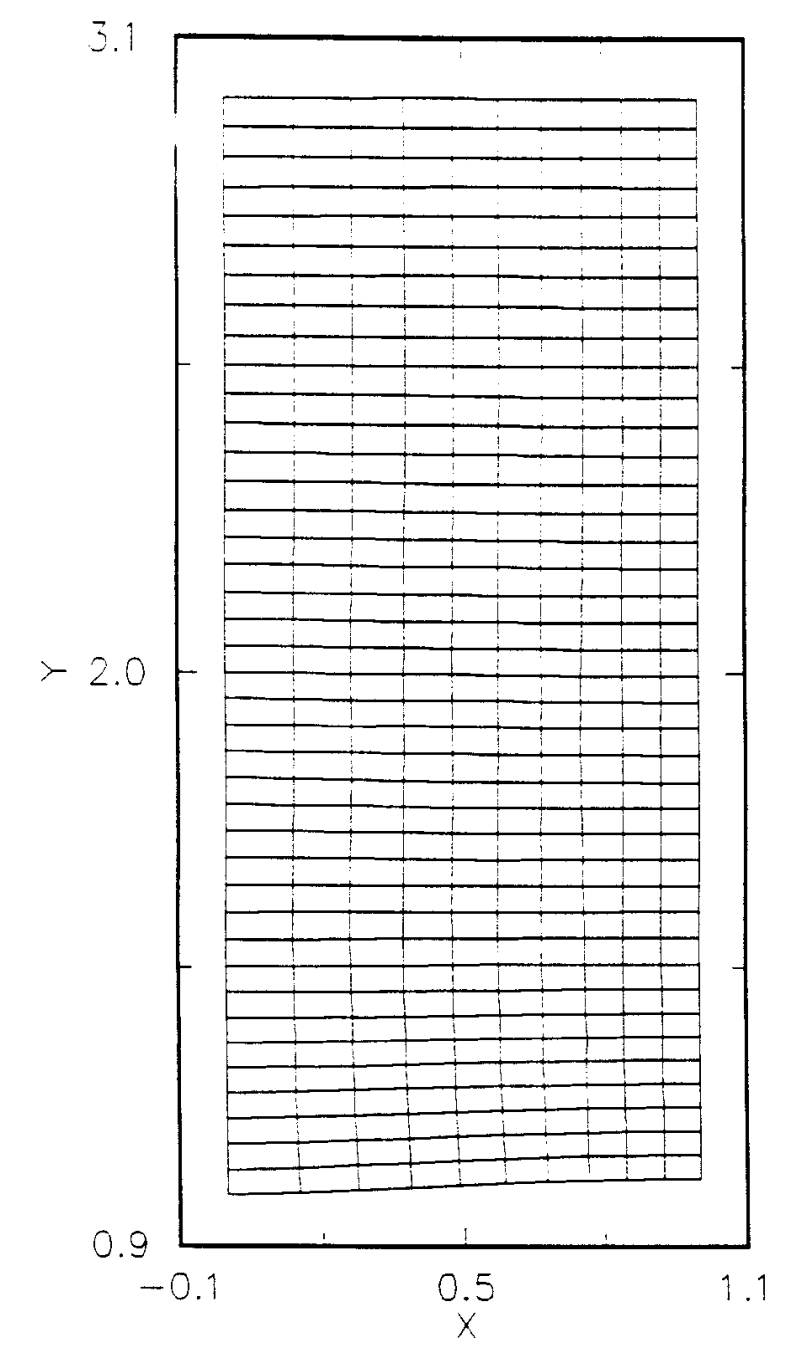

(a)

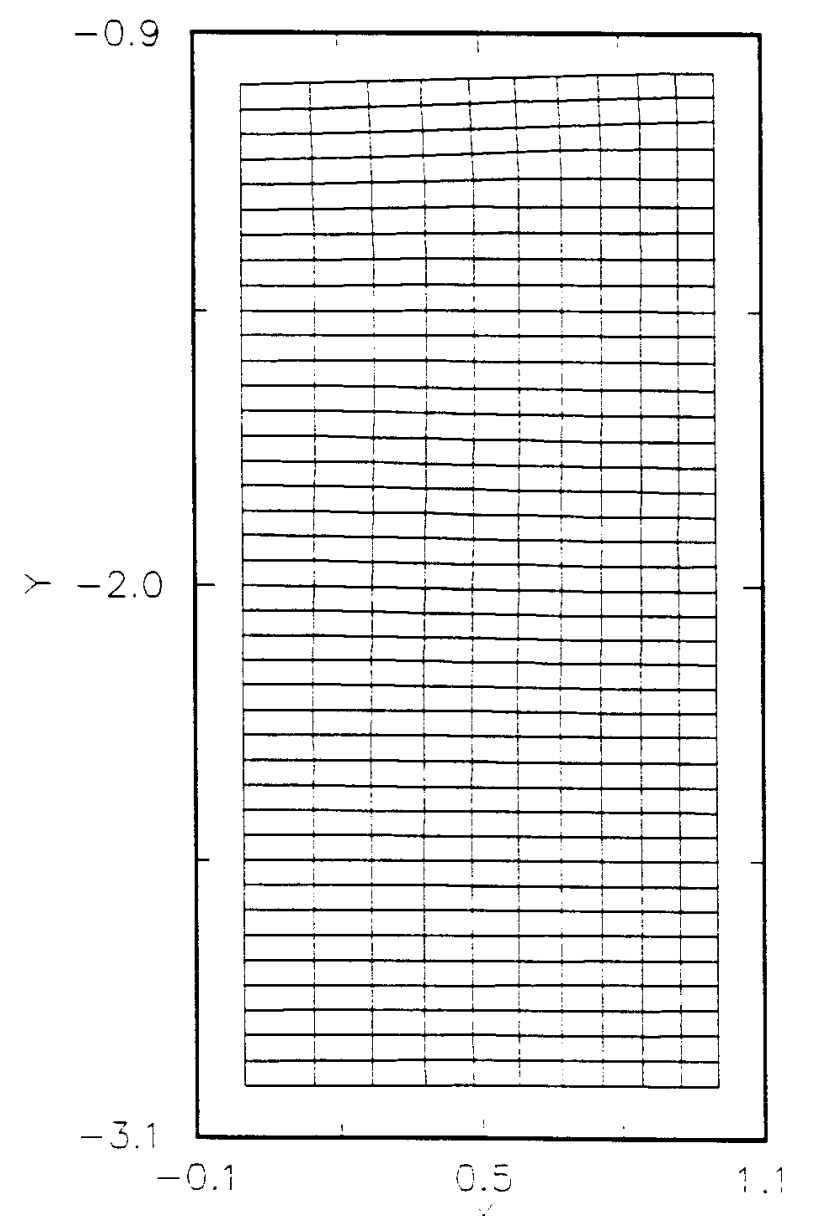

(b)

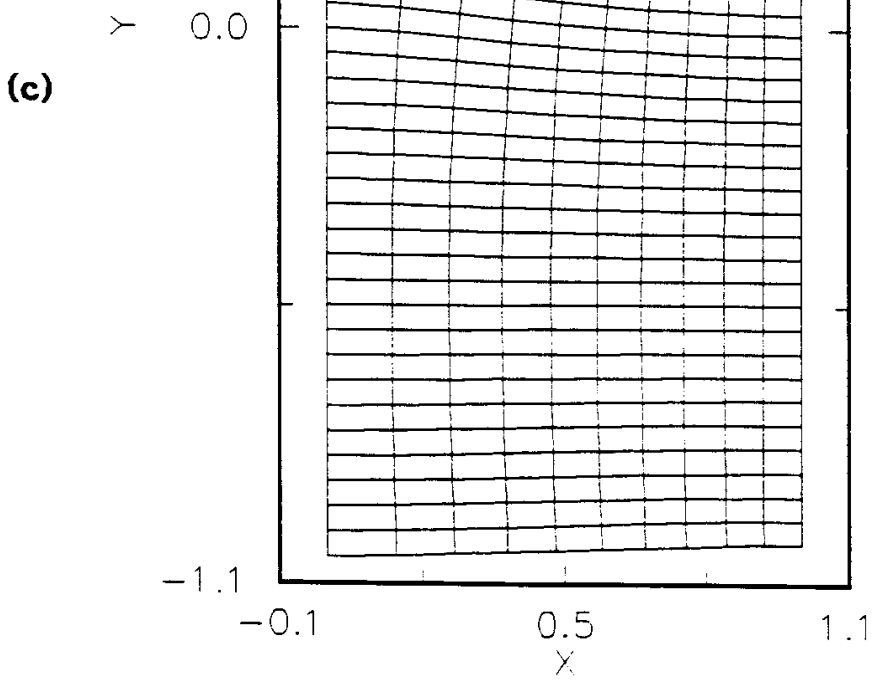

FIG. -7.2 


\section{Sugestões para Trabalho Futuro}

1. Quanto à constatação feita no final da sec. 6.4 do cap. VI de que - cálculo numérico então empregado para a determinação da distribuição a( $\psi)$ fornece em geral valores maiores do que os esperados, propõe-se a seguinte revisão: após obter-se uma solução numérica para todas as grandezas pertinentes via convergência do Algoritmo Il (ver cap.V, sec. 5.3), os valores de a( $\psi$ ) sejam corrigidos, em principio, através do emprego combinado das eqs. (6.5) e (6.6). Pela primeira dessas equação calcula-se $E(\Phi, \psi)$ em cada ponto nodal "e" de cada linha de fluxo $(\psi=c t e$.$) . Os valores obtidos serão transportados para a segunda equação$ que por seu turno permitirá recalcular um valor médio para $a(\Psi)$ sobre as linhas consideradas. Estas operações definiriam um $12^{\circ}$ passo no Algoritmo II que poderia ser repetido convenientemente algumas vezes. Nessa fase e/ou precedentemente, o procedimento de Stone [25] poderia ser implementado para economia de tempo. Poder-se-ia também aumentar o número de pontos na discretização da equação que vimos usando para o cálculo iterativo de $a(w)$ (eq. (4.21)) e experimentar valores do espaçamento de grade maiores e menores do que $\mathrm{h}=0.05$.

2. Empregar um outro método numérico tal como o de Newton e Raphson em que $x(\Phi, \psi), y(\Phi, \psi)$ e $a(\psi)$ seriam tratados como as variáveis, obtendo assim novos resultados para uma comparação com os presentes.

3. Estudar numericamente o comportamento das distribuições de carga espacial como uma função da distância tomada ao longo das linhas de corrente. Uma hipótese inicial de trabalho é supor que essa função é do tipo da que se determina exatamente no problema do fluxo de carga entre dois eletródios planos, paralelos e infinitos, produzido por injeção uniforme de carga espacial.

4. Recentemente [28] fizemos um cálculo aproximado e intuitivo de linhas de corrente extremas, $\partial \Gamma$, em um sistema de eletródios e em condição de injeção de carga espacial ambos semelhantes aos descritos na Fig.-5.1(a). Consideramos, de início, uma parede impermeável às cargas colocada em $y=0$, de 
$x=0$ até $x=a$ (esse caso os eletródios estavam separados por $\Delta x=a$ e não $\Delta x=1$ como na Fig. -5.1(a)). A componente de campo, $E_{x L}(x, 0)$, tangente à parede, foi deduzida supondo que na região de Poisson e devido à carga superficial acumulada na parede, o campo fosse como num dielétrico infinito sob injeção uniforme de carga por toda a extensão do eletródio (emissor); a compomente transversal, $E_{y L}(x, 0)$, foi calculada pelo uso da função de Green do tipo Dirichlet para o problema da semibanda definida pela região de Laplace. Na ausência da parede o campo e a distribuição de carga espacial são distorcidos. A carga injetada penetra numa faixa do que antes era região de Laplace e delimita uma linha de corrente extrema. Sugerimos, intuitivamente, que nessa linha as cargas se deslocariam sob a ação de um campo cujas componentes eram ainda dadas por $E_{x L}(x, 0)$ e $E_{y L}(x, 0)$, sendo o perfil da linha descrito por $d y / d x=E_{y L}(x, 0) / E_{x L}(x, 0)$. Propõe-se então que esses cálculos sejam refeitos considerando as mesmas densidades de carga e as mesmas grandezas adimensionais usadas nesta tese para mais uma comparação com os resultados presentes. Poder-se-ia resolver no caso contemplado neste trabalho, um problema de Laplace dando em uma das linhas de campo limites $\left(\psi=\psi_{1}\right)$ do domínio a função $x\left(\Phi, \psi_{1}\right)$ correspondente à situação de carga espacial em simetria planar e, na outra (linha limite oposta), a de campo uniforme. Da solução resultaria a função $y\left(x\left(\Phi, \psi_{1}\right)\right)$ que não deverá, espera-se, diferir significativamente dos resultados (วГ's) obtidos pelos diferentes métodos. Será mesmo assim?

5. Estender o método ao estudo de outras situações de interesse. Considerar, por exemplo, o problema de simetria cilindrica em que um fluxo estacionário de carga espacial em um condensador plano tem por fonte um círculo de raio a sobre um dos eletródios. As linhas de campo limites do domínio de solução seriam o eixo de simetria, em $r=0$, e uma linha de campo laplaciano em $r \gg a$. Por último, os efeitos da emissão descontinua de carga espacial sobre as linhas de campo seriam investigados em outros eletródios não planos. 


\section{APÊNDICE-A}

\section{Sobre o Emprego das Sub-Rotinas NAG F04AXF e F01BRF}

O uso das sub-rotinas NAG F04AXF e F01BRF para calcular soluções aproximadas de um sistema de equações reais lineares esparças da forma $\mathbb{M X}=\mathbb{B}$, da qual as eqs. (4.19a,b) (sec.4.4, cap.IV) são um exemplo, é muito simples quando a matriz dos coeficientes, $M$, tem pequena dimensão. Com o crescimento do número de elementos não nulos da matriz o emprego dessas sub-rotinas torna-se algo mais elaborado e por esta razão oferecemos aqui um esquema para tratar especificamente com equações lineares do tipo descrito pelas eqs. $(4.19 a, b)$.

A sub-rotina F04AXF resolve o sistema de equações $M X-\mathbb{B}$ por substituição para frente e para trás enquanto F01BRF (e/ou F01BSF), precedentemente, faz a decomposição $\mathbb{L} U$ de uma permutação da matriz $M$, $\mathbb{P} M Q=\mathbb{L} U$, em que $\mathbb{P}$ e $\mathbb{Q}$ são matrizes de permutação (para maiores detalhes ver documento de implementação que acompanha a biblioteca NAG).

Entre os muitos parâmetros ou argumentos (consultar Apend.-B), estamos particularmente interessados em construir $\underline{\mathbb{N}} \underline{\mathbb{N} Z} \underline{\mathbb{A}} \underline{\mathbb{I R} \mathbb{N}} \underline{\mathbb{I C N}}$ na sub-rotina $\operatorname{F01BRF}(\mathbb{N}, \mathbb{N Z}, \mathbf{A}, \mathbb{I R N}, \mathbb{I C N}, \cdots)$ e $\underline{\mathbb{R} H S}$ na sub-rotina F04AXF(N, N, RHS, ...). Eis o seu significado quando da entrada:

$\mathbb{N}$ - inteiro que especifica a ordem da matriz $\mathbb{M}$;

$\mathbb{N Z}$ - inteiro que especifica o número de elementos não nulos da matriz $\mathbb{M}$;

A - arranjo unidimensional contendo, em qualquer ordem, os $\mathbb{N} Z$ elementos não nulos da matriz $\mathbb{M}$;

$\mathbb{I R N}$ - arranjo inteiro unidimensional contendo os indices de linhas dos elementos não nulos estocados em $\mathbf{A}$;

ICN - arranjo inteiro unidimensional contendo os índices de colunas dos elementos não nulos estocados em $\mathbf{A}$;

$\mathbb{R H S}$ - arranjo unidimensional contendo as $\mathbb{N}$ componentes do vetor coluna $\mathbb{B}$ dos 
termos independentes do sistema de equações. Na saida RHS contém as componentes do vetor solução $\mathbf{X}$.

Tomemos em conta, no que se segue, o domínio discretizado da Fig. 4.2 (cap.IV). A eq. (4.19a) será aplicada a cada nodo interno "•" para o cômputo da aproximação $\hat{X}(i, j)$. Há $(N / 2-1)$ desses nodos na direção $\Phi,(M / 2-1)$ na direção $\Psi$ e o total deles, isto é, o número de equações do sistema resultante ou ordem da matriz é, pois,

$$
\mathbb{N}=(\mathrm{N} / 2-1)(\mathrm{M} / 2-1),
$$

onde $N$ e $M$ estão explicitados na Fig. -4.2 .

Considerando que os nodos "•" são varridos sistematicamente da esquerda para a direita e de baixo para cima, a matriz $M$ dos coeficientes das equaçōes lineares resultantes tem a seguinte caracterização' ${ }^{1}$ é uma matriz esparsa cujos elementos não nulos estão arranjados ao longo de cinco diagonais a diagonal principal (DP), duas diagonais superiores (DS1 e DS2) e duas diagonais inferiores (DI1 e DI2) - como sugere o esquema da Fig. - A.1

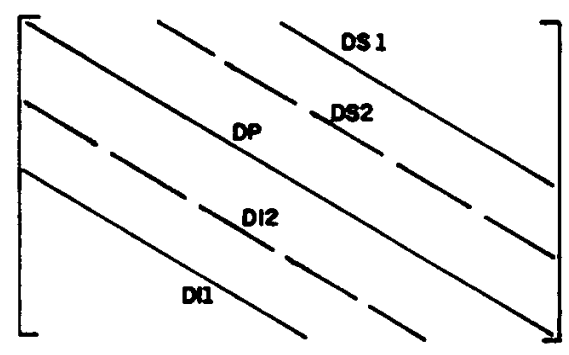

FIG.-A.1 Representação esquemática da matriz $M$ dos coeficientes das equações lineares $(4.19 a, b)$. As linhas sugerem a posição dos elementos não nulos e os brancos a dos zeros da matriz.

$\underline{\text { DS1 }}$

Número de elementos (NDS1): NDS1 $=(\mathrm{N} / 2-1)(\mathrm{M} / 2-2)$.

Posição $[I ; J]^{2}$ na matriz:

${ }^{1} \mathrm{Na}$ prática construímos uma matriz $\mathbb{M} 25 \times 25$. levantamos suas características a as generalizamos para uma matriz $((N / 2-1)(M / 2-1) \times(N / 2-1)(M / 2-1)]$ por induçāo matomátıca.

${ }^{2} \underline{I} \odot \underline{J}$ são os mosmos que $\underline{i}$ e j na Fig.-4.2 
INÍCIO $\rightarrow[1 ; \mathrm{N} / 2]$

FIM $\rightarrow[(\mathrm{N} / 2-1)(\mathrm{M} / 2-2) ;(\mathrm{N} / 2-1)(\mathrm{M} / 2-1)]$

Expressão genérica para os elementos $\left(\alpha_{1}(\mathrm{I}, \mathrm{J}+1)\right)$ :

$\alpha_{1}(\mathrm{I}, \mathrm{J}+1)=\frac{2}{\mathrm{~h}^{2}}[\mathrm{a}(\mathrm{J}+1)(\sigma(\mathrm{I}-1, \mathrm{~J}+1)+\sigma(\mathrm{I}+1, \mathrm{~J}+1))]^{-1}, \quad \mathrm{~J}=2(\mathrm{M}-4) 2 \quad$ e $\quad \mathrm{I}=2(\mathrm{~N}-2) 2 .^{3}$

DI1

Número de elementos (NDI2): NDI2 $=(\mathrm{N} / 2-1)(\mathrm{M} / 2-2)$.

Posição $[1 ; J]$ na matriz:

INÍCIO $\rightarrow[\mathrm{N} / 2 ; 1]$

FIM $\rightarrow[(\mathrm{N} / 2-1)(\mathrm{M} / 2-1) ;(\mathrm{N} / 2-1)(\mathrm{M} / 2-2)]$.

Expressão genérica para os elementos $\left(\alpha_{3}(I, J-1)\right)$ :

$\alpha_{3}(\mathrm{I}, \mathrm{J}-1)=\frac{2}{\mathrm{~h}^{2}}[\mathrm{a}(\mathrm{J}-1)(\sigma(\mathrm{I}-1, \mathrm{~J}-1)+\sigma(\mathrm{I}+1, \mathrm{~J}-1))]^{-1}, \quad \mathrm{~J}=4(\mathrm{M}-2) 2 \quad$ e $\quad \mathrm{I}=2(\mathrm{~N}-2) 2$.

DS2

Esta diagonal possui $(M / 2-1)$ segmentos intercalados por um zero, contendo $(\mathrm{N} / 2-2)$ elementos (não nulos) cada.

Número de elementos (NDS2): $\quad$ NDS2 $=(\mathrm{N} / 2-2)(\mathrm{M} / 2-1)$.

Posição [I; J] de cada um dos $(M / 2-1)$ segmentos (SDS2(K)) da diagonal DS2 na matriz $-\operatorname{SDS} 2(\mathrm{~K}), \quad \mathrm{K}=1(\mathrm{M} / 2-1) 1:$

INICIO $\rightarrow[[(\mathrm{K}-1)(\mathrm{N} / 2-1)+1] ;[(\mathrm{K}-1)(\mathrm{N} / 2-1)+2]]$,

FIM $\quad \rightarrow \quad[[K(N / 2-1)-1] ;[K(N / 2-1)]]$.

Expressão genérica para os elementos $\left(\alpha_{2}(\mathrm{I}+2,2 \mathrm{~K})\right)$ :

$\alpha_{2}(\mathrm{I}+2,2 \mathrm{~K})=\frac{1}{2 \mathrm{~h}^{2}}[\mathrm{a}(2 \mathrm{~K}-1) \sigma(\mathrm{I}+1,2 \mathrm{~K}-1)+\mathrm{a}(2 \mathrm{~K}+1) \sigma(\mathrm{I}+1,2 \mathrm{~K}+1)], \quad \mathrm{I}=2(\mathrm{~N}-4) 2 \quad(\mathrm{~J}=2 \mathrm{~K})$.

\section{$\underline{\text { DI2 }}$}

Como no caso de DS2 esta diagonal possui também (M/2-1) segmentos intercalados por um zero, contendo $(\mathrm{N} / 2-2)$ elementos cada.

Número de elementos (NDI1): NDI1 $=(\mathrm{N} / 2-2)(\mathrm{M} / 2-1)$.

Posição [I;J] de cada um dos $(M / 2-1)$ segmentos ( SDI1(K) ) da diagonal DI1 na matriz - $\operatorname{SDI1}(\mathrm{K}), \quad \mathrm{K}=1(\mathrm{M} / 2-1) 1$ : 
INICIO $\rightarrow \quad[[(\mathrm{k}-1)(\mathrm{N} / 2-1)+2] ;[(\mathrm{K}-1)(\mathrm{N} / 2-1)+1]]$,

FIM $\rightarrow[[K(N / 2-1)] ;[K(N / 2-1)-1]]$.

Expressão genérica para os elementos $\left(\alpha_{4}(\mathrm{I}-1,2 \mathrm{~K})\right)$ :

$\alpha_{4}(\mathrm{I}-1,2 \mathrm{~K})=\frac{1}{2 \mathrm{~h}^{2}}[\mathrm{a}(2 \mathrm{~K}-1) \sigma(\mathrm{I}-1,2 \mathrm{~K}-1)+\mathrm{a}(2 \mathrm{~K}+1) \sigma(\mathrm{I}-1,2 \mathrm{~K}+1)], \quad \mathrm{I}=4(\mathrm{~N}-2) 2 \quad(\mathrm{~J}=2 \mathrm{~K})$.

$\underline{\mathrm{DP}}$

Número de elementos $(N D P): \quad N D P=(N / 2-1)(M / 2-1)$.

Posição $[1 ; J]$ na matriz:

INICIO $\rightarrow[1 ; 1]$

FIM $\rightarrow[(N / 2-1)(M / 2-1) ;(N / 2-1)(M / 2-1)]$.

Expressão genérica para os elementos $(\mathrm{A}(\mathrm{I}, \mathrm{J}))$ :

$\mathrm{A}(\mathrm{I}, \mathrm{J})=-\left[\alpha_{1}(\mathrm{I}, \mathrm{J}+1)+\alpha_{2}(\mathrm{I}+2, \mathrm{~J})+\alpha_{3}(\mathrm{I}, \mathrm{J}-1)+\alpha_{4}(\mathrm{I}-1, \mathrm{~J})\right], \quad \mathrm{J}=2(\mathrm{M}-2) 2 \quad$ e $\quad \mathrm{I}=2(\mathrm{~N}-2) 2$.

NÚMERO DE ELEMENTOS NÃO NULOS DA MATRIZ (NZ) ${ }^{4}$

$\mathbb{N Z}=\mathrm{NDS} 1+\mathrm{NDI} 2+\mathrm{NDS} 2+\mathrm{NDI} 1+\mathrm{NDP}=\frac{5}{4} \mathrm{NM}-\frac{7}{2}(\mathrm{~N}+\mathrm{M})+9$.

Indexemos na forma $X(K)$ o vetor solução da equação matricial

$\mathbb{M X}=$ B. A dimensão de $\mathbf{X}(K)$ é, obviamente, a ordem da matriz, ou seja, $\mathrm{K}=1,2,3, \ldots,(\mathrm{N} / 2-1)(\mathrm{M} / 2-1)$. A transformação das componentes de $\mathrm{X}(\mathrm{K})$ para os elementos da aproximação $\hat{\mathrm{X}}(I, J)$ é dada pelo seguinte fluxograma:

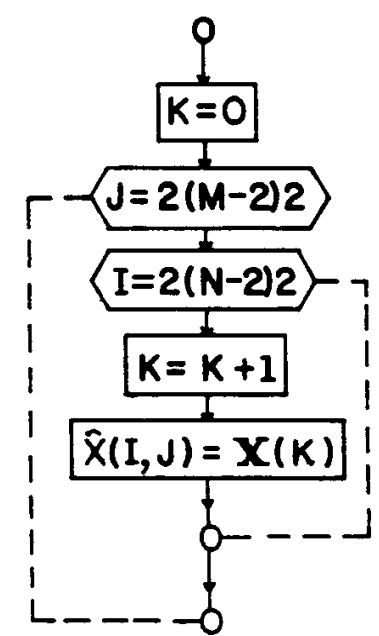


$O$ vetor dos termos independentes, $B$, possui $(M / 2-1)$ segmentos verticais com (N/2-1) elementos (nulos e não nulos) cada. O primeiro segmento (ou superior) reúne as condições de contorno na base do domínio discretizado, o último segmento está relacionado com as condiçōes no contorno do topo e os segmentos intermediários, no que concerne aos elementos não nulos, têm relação tão somente com as condições nas fronteiras $\Phi=1$ e $\Phi=0$ do dominio. As componentes de $B(K)$ e

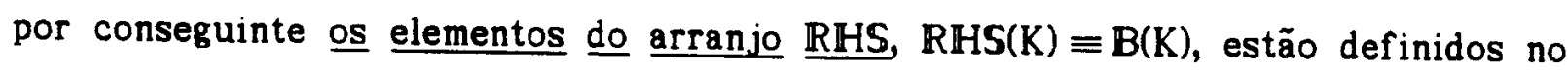
fluxograma abaixo, com $\mathrm{K}=1,2,3, \ldots,(\mathrm{N} / 2-1)(\mathrm{M} / 2-1)$.

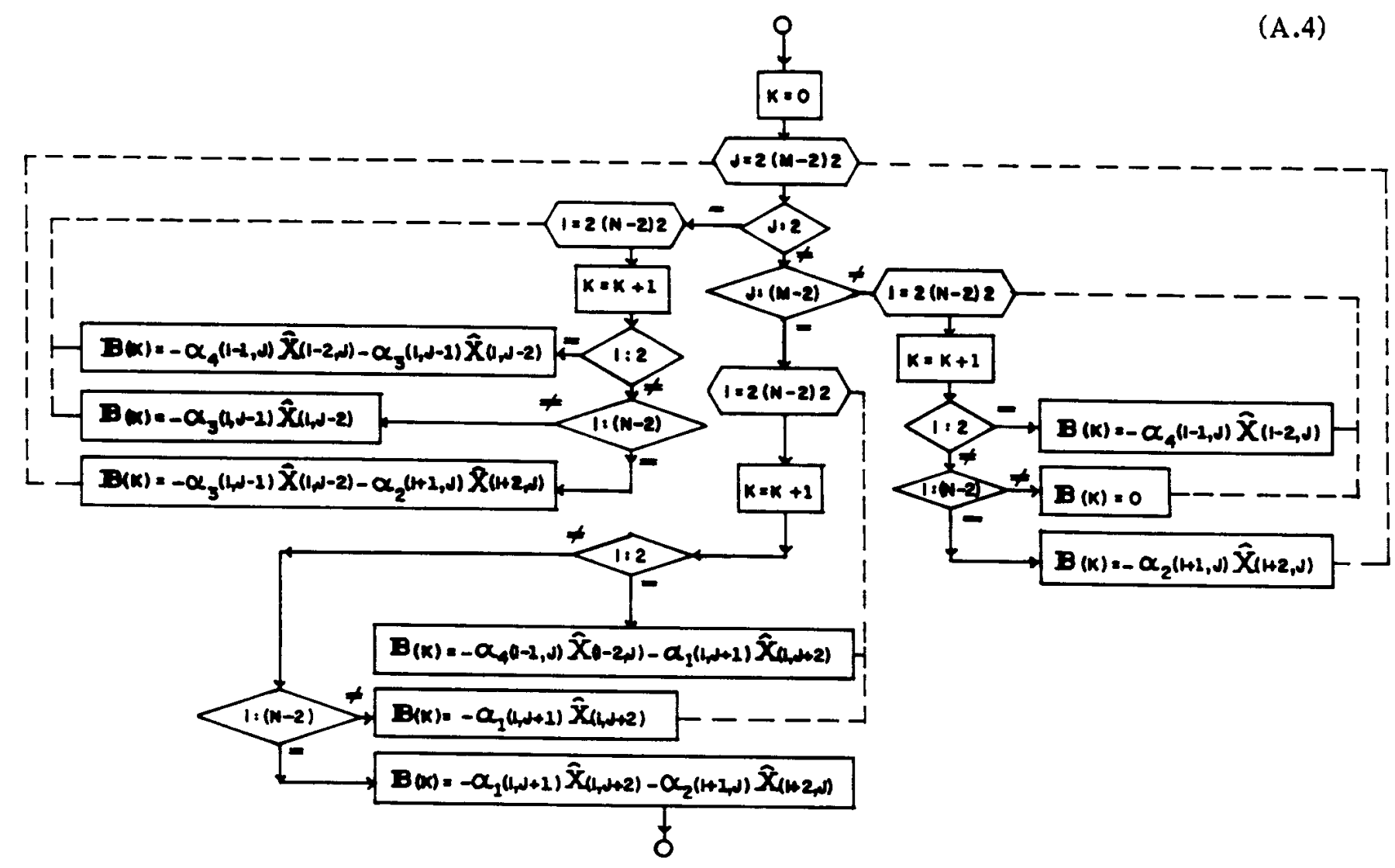

O arranjo, $\mathbf{A}$, dos elementos não nulos da matriz, é construído (poderia ser diferente) numa ordenação linear tal que,

$$
A(\kappa)=(\mathrm{DS} 1(\mathrm{~K} 1), \mathrm{DI} 2(\mathrm{~K} 2), \mathrm{DP}(\mathrm{K} 3), \mathrm{DS} 2(\mathrm{~K} 4), \mathrm{DI} 1(\mathrm{~K} 5))
$$

$\operatorname{com} \quad \kappa=1(\mathbb{N Z}) 1 ; \quad \mathrm{K} 1=1(\mathrm{NDS} 1) 1 ; \quad \mathrm{K} 2=1(\mathrm{NDI} 2) 1 ; \quad \mathrm{K} 3=1(\mathrm{NDP}) 1 ; \quad \mathrm{K} 4=1(\mathrm{NDS} 2) 1 ;$ K5=1(NDI1)1. Forçosamente a ordenação dos índices de linhas e colunas nos arranjos $\mathbb{I} \mathbb{R}(\kappa)$ e $\mathbb{I C N}(\kappa)$ será a mesma definida em $A(\kappa)$. 
A construção de $\mathbf{A}(\kappa), \mathbb{R} \mathbb{N}(\kappa)$ e $\mathbb{I C N}(\kappa)$ está dada no fluxograma que segue.

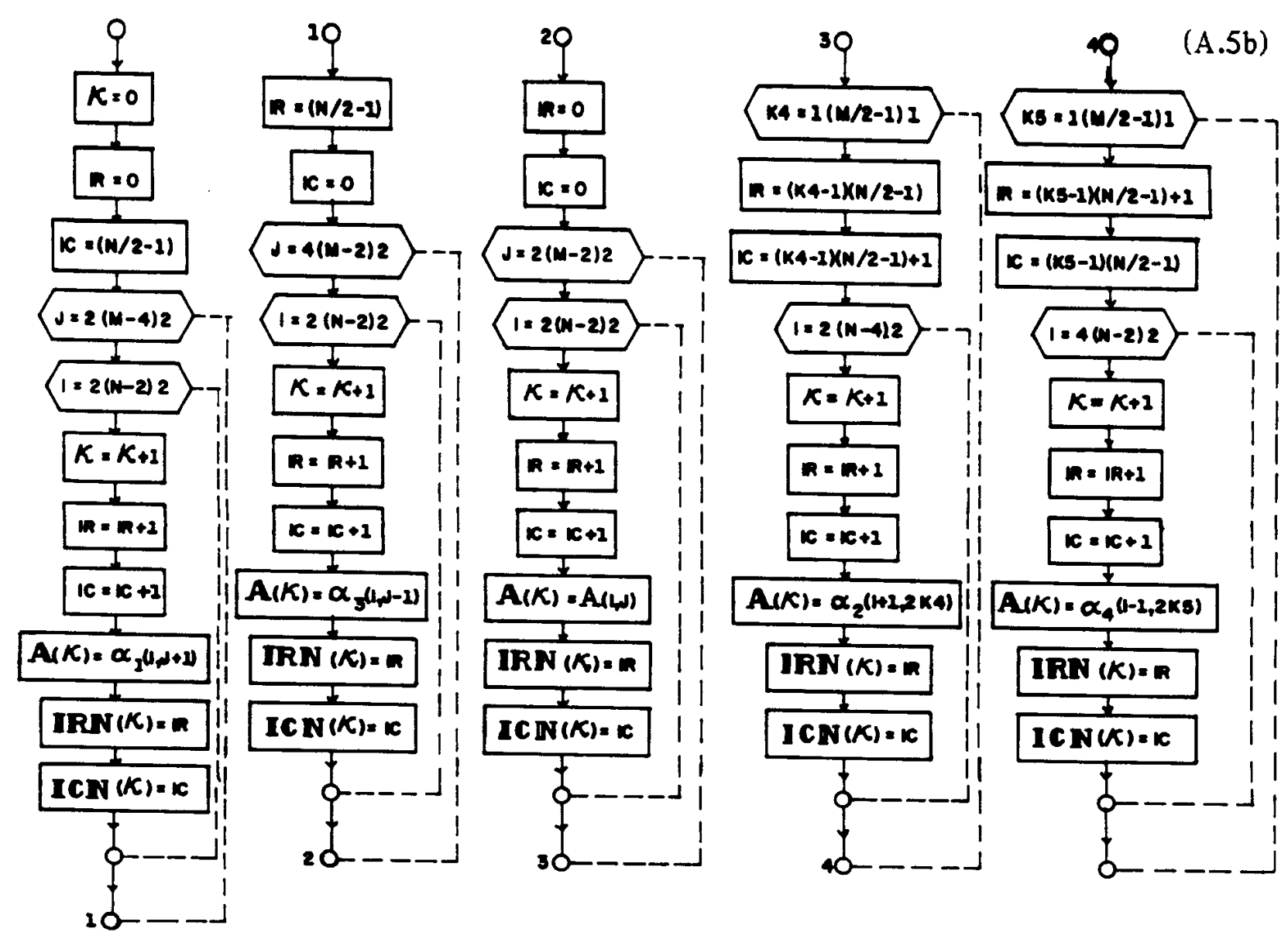




\section{AP E $N D I C E-B$}

\section{PROGRAMAS FORTRAN}

\section{Programa Gross}

\section{PROGRAMA GROSS. FOR}

\section{*********************}

Este programa resolve numericamente as equações de carga espacial sob a transformação hodográfica de Budd \& Wheeler [4] em um domínio simplesmente conexo. Usa um método iterativo simples e emprega a biblioteca NAG para a resolução do sistema de equacões lineares da dicra da discretização das equações. O programa determina a fronteira de choque que subdivide o dominio em dois subdominios, SUBDOM2 e SUBDOM1, respectivamente. Há carga espacial no primeiro subdomínio e inexiste no segundo. A fronteira conecta dois eletródios ou equipotenciais.

PARAMETER ( $\mathrm{N}=40, \mathrm{M}=160, \mathrm{NZ}=7309, \mathrm{NEQ}=1501)$

PARAMETER ( $L I C N=80000$, LIRN $=60000)$

$\mathrm{C}$

PARAMETER ( PSII $=-4$. , PSIF $=4$. )

REAL SIGG(0:(N-1),1:(2*M-1)),XG(0:N,0:(2*M)), YG(0:N,0:(2*M)), * APSIG $(1:(2 * M-1))$, RHOO

C INTEGER ITEREX ,IDOM1, IDOM2,NXGIMB

... "G" nas grandezas acima quer dizer que elas são globais para ... os dois subdominios, "Subdom1" e "Subdom2".

TYPE '(A)','\$ WSIG (PESO P/SIG), TOLSIG (TOLER.P/SIG):' ACCEPT $*$,WSIG,TOLSIG

TYPE '(A)','S WX (PESO P/X), TOLX (TOLER.P/X):' ACCEPT *,WX,TOLX

TYPE '(A)','S WAP (PESO P/APSI), TOLAP (TOLER.P/APSI):' ACCEPT $*$,WAP,TOLAP

TYPE '(A)','S RHOO:'

ACCEPT *,RHOO

$\mathrm{H}=2 . / \mathrm{N}$

C
C
C
C
C
C

$\mathrm{HP}=\mathrm{H}$

$\mathrm{NZ}=5 *((\mathrm{~N} * \mathrm{M}) / 4)-7 *((\mathrm{~N}+\mathrm{M}) / 2)+9$

$\mathrm{NEQ}=(\mathrm{N} / 2-1) *(\mathrm{M} / 2-1)$

$\mathrm{LICN}=10 * \mathrm{NZ}$

LIRN $=$ INT $(8 . * N Z)$

$\mathrm{A} 0=1$.

$\mathrm{A} 1=2 . *$ RHOO -1 .

$\mathrm{A} 2=4 . * \mathrm{RHOO} * \mathrm{RHOO} / 3$.

$\mathrm{A} 3=-3 . * \mathrm{RHO} / 2$.

$\mathrm{HO}=(3 . * \mathrm{~A} 0 * \mathrm{~A} 2-\mathrm{A} 1 * \mathrm{~A} 1) /(9 . * \mathrm{~A} 0 * \mathrm{~A} 0)$

$\mathrm{G}=2 . *((\mathrm{~A} 1 /(3 . * \mathrm{~A} 0)) * * 3)-\mathrm{A} 1 * \mathrm{~A} 2 /(3 . * \mathrm{~A} 0 * \mathrm{~A} 0)+\mathrm{A} 3 / \mathrm{A} 0$

$\mathrm{U} 0=(-\mathrm{G}+\mathrm{SQRT}(\mathrm{G} * \mathrm{G}+4 * * \mathrm{H} 0 * \mathrm{H} 0 * \mathrm{H} 0)) / 2$.

$\mathrm{U} 13=\mathrm{U} 0 * *(1 . / 3$. $)$

$\mathrm{E} 0=\mathrm{U} 13-\mathrm{H} 0 / \mathrm{U} 13-\mathrm{A} 1 /(3 . * \mathrm{~A} 0)$

$\mathrm{C} 1 \mathrm{~S}=\mathrm{E} 0 /(2 . * \mathrm{RHOO})$ 
C

C

$\mathrm{C}$

C

10

DO $10 \mathrm{~J}=1,(\mathrm{M}-1), 2$

$\operatorname{SIGG}(0, J)=1$.

$\operatorname{SIGG}(0, \mathrm{~J}+\mathrm{M})=1 . / \mathrm{RHO} 0$

DO $20 \mathrm{I}=0, \mathrm{~N}, 2$

$\mathrm{FI}=1 .-(\mathrm{I} / 2) * \mathrm{H}$

$\mathrm{XG}(\mathrm{I}, 0)=1 .-\mathrm{FI}$

$\mathrm{XG}(\mathrm{I}, 2 * \mathrm{M})=\mathrm{C} 1 \mathrm{~S} *((\mathrm{C} 2 \mathrm{~S} *(1 .-\mathrm{FI})+1) * *(2 . / 3)-1$.

$Y G(I, 0)=P S I I$

20

$\mathrm{YG}(\mathrm{I}, 2 * \mathrm{M})=\mathrm{PSIF}$

CONTINUE

DO $30 \mathrm{~J}=0,(2 * \mathrm{M}), 2$

$\mathrm{PSI}=\mathrm{PSII}+\mathrm{J} * \mathrm{HP} / 2$.

$\mathrm{YG}(0, \mathrm{~J})=\mathrm{PSI}$

$X G(0, J)=0$.

$\mathrm{XG}(\mathrm{N}, \mathrm{J})=1$.

30

CONTINUE

C

C

$\mathrm{C}$

C

FUNÇÕES TENTATIVA PARA SIG, X E APSI:

DO $40 \mathrm{~J}=1,(\mathrm{M}-1), 2$

$\operatorname{APSIG}(\mathrm{J})=-1$.

$\operatorname{APSIG}(\mathrm{J}+\mathrm{M})=-\mathrm{RHO} * \mathrm{EO}$

DO $50 \mathrm{I}=1,(\mathrm{~N}-1), 2$

$\mathrm{FI}=1 .-\mathrm{I} * \mathrm{H} / 2$.

SIGGI2M- $((\mathrm{C} 2 \mathrm{~S} *(1 .-\mathrm{FI})+1) * *.(1 . / 3).) / \mathrm{RHOO}$

$\operatorname{SIGG}(I, J)=1$.

50

$\operatorname{SIGG}(I, J+M)=\operatorname{SIGGI} 2 M$

CONTINUE

40 CONTINUE

DO $60 \mathrm{I}=2,(\mathrm{~N}-2), 2$

$\mathrm{SLOPE}=(\mathrm{XG}(1,2 * \mathrm{M})-\mathrm{XG}(\mathrm{I}, 0)) /(\mathrm{PSIF}-\mathrm{PSII})$

DO $70 \mathrm{~J}=2,(2 * \mathrm{M}-2), 2$

$\mathrm{PSI}=\mathrm{PSII}+(\mathrm{J} / 2) * \mathrm{HP}$

$70 \quad$ CONTINUE

60 CONTINUE

ITEREX $=0$

80 ITEREX $=$ ITEREX +1

IDOM $1=0$.

IDOM $2=0$.

CALL SUBDOM1(SIGG,APSIG,XG,YG,IDOM1,

*

WX,TOLX,WAP,TOLAP

IF(IDOM1.EQ.1) GO TO 180

CALL SUBDOM2(SIGG,APSIG,XG,YG,IDOM2,

* WSIG,TOLSIG, WX, TOLX,WAP, TOLAP)

C IF(IDOM2.EQ.1) GO TO 180

C ... Calculo iterativo de XG(I,M) - Fronteira de Choque.

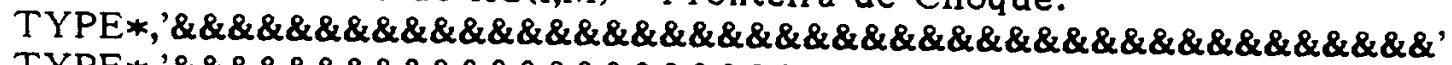

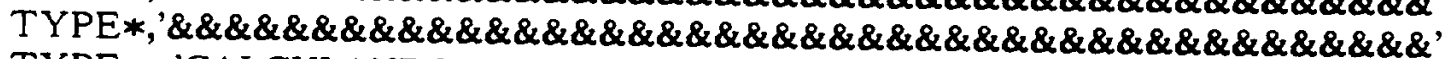
TYPE $*$,'CALCULANDO XG(I,J) NA FRONTEIRA.\&\&\&\&\& ITEREX=',ITEREX

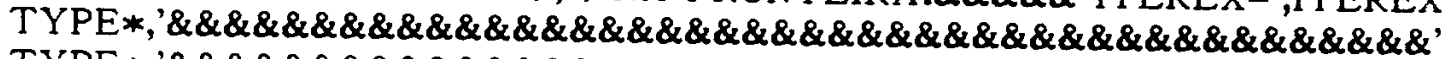

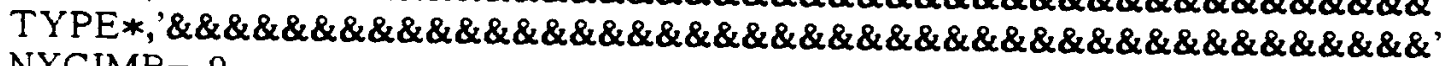
$\mathrm{NXGIMB}=0$.

DO $90 \mathrm{I}=2,(\mathrm{~N}-2), 2$

APSIGG1 $=1.5 *$ APSIG(M-1)-.5*APSIG(M-3) 
APSIGG $2=.75 *$ APSIG $(M+1) *(\operatorname{SIGG}(1+1, M+1)+\operatorname{SIGG}(1-1, M+1))-$

* $\quad .25 * A P S I G(M+3) *(\operatorname{SIGG}(I+1, M+3)+\operatorname{SIGG}(I-1, M+3))$

C XGIMAT $=($ APSIGG $1 * X G(1, M+2)+A P S I G G 2 * X G(I, M-2)) /(A P S I G G 1+A P S I G G 2)$

$\mathrm{XGIMNOVO}=.25 * \mathrm{XG}(\mathrm{I}, \mathrm{M})+.75 * \mathrm{XGIMAT}$

DELXGIM= ABS(XGIMNOVO-XG(I,M))

C IF(DELXGIM.LE.TOLX) NXGIMB $=\mathrm{NXGIMB+1}$

IF(DELXGIM.LE..0005) NXGIMB=NXGIMB+1

90 CONTINUE

APROXGIM $=(\operatorname{REAL}(\mathrm{NXGIMB}) /(\operatorname{REAL}(\mathrm{N} / 2-1))) * 100$.

IF(ITEREX.LT.12) THEN

IF(APROXGIM.LT.100.) THEN

GO TO 80

ENDIF

GO TO 100

ENDIF

IF(APROXGIM.LT.100.) THEN

TYPE '(A)',' $\$$ NUMERO DE ITERACOES PARA XG(I,M), ITEREX, =12'

TYPE '(A)','S NAO HA CONVERGENCIA PARA XG(I,M)'

TYPE '(A)','S TENTE NOVOS PESOS E TOLERANCIAS'

GO TO 180

ENDIF

C

...Calculo de YG(I,M) - FRONTEIRA de CHOQUE.

DO $110 \mathrm{I}=2,(\mathrm{~N}-2), 2$

APSIGG $1=1.5 *$ APSIG $(M-1)-.5 *$ APSIG(M-3)

APSIGG $2=.75 * \operatorname{APSIG}(M+1) *(\operatorname{SIGG}(1+1, \mathrm{M}+1)+\operatorname{SIGG}(1-1, \mathrm{M}+1))-$

* . 25*APSIG $(M+3) *(\operatorname{SIGG}(1+1, \mathrm{M}+3)+\operatorname{SIGG}(\mathrm{I}-1, \mathrm{M}+3))$

110 CONTINUE

$\mathrm{YG}(\mathrm{N}, \mathrm{M})=\mathrm{YG}((\mathrm{N}-2), \mathrm{M})$

C

C

C

...choque.

...Resultados para $a(\psi)$ e $E(1, \psi)$ :

DO $120 \mathrm{~J}=1,(\mathrm{M}-1), 4$

$\mathrm{PSI}=\mathrm{PSII}+\mathrm{J} * \mathrm{HP} / 2$.

CAMP $=$ ABS(APSIG(J))

WRITE $(20, *)$ PSI,CAMP

120 CONTINUE

C

DO $130 \mathrm{~J}=1,(\mathrm{M}-1), 8$

$\mathrm{PSI}=\mathrm{J} * \mathrm{HP} / 2$.

C WRITE $(20, *)$ PSI, APSIG $(J+M)$

C130 CONTINUE

C

DO $130 \mathrm{~J}=1,(\mathrm{M}-1), 4$

$\mathrm{PSI}=\mathrm{J} * \mathrm{HP} / 2$.

$\mathrm{CAMP}=\operatorname{ABS}(\operatorname{APSIG}(J+\mathrm{M}) * \operatorname{SIGG}(0, J+M))$

WRITE $(20, *)$ PSI,CAMP

130 CONTINUE

C $\quad$...Resultados para $\sigma(\Phi, \psi)$ e lou $\sigma(\mathrm{X}, \mathrm{Y})$ :

DO $150 \mathrm{~J}=1,(\mathrm{M}-1), 8$

C PSI $=\mathrm{J} * \mathrm{HP} / 2$.

C WRITE(10,*) PSI, (1./SIGG $(I, J+M))$

C150 CONTINUE

C140 CONTINUE

C

DO $140 \mathrm{~J}=1,(\mathrm{M}-1), 2$

$\mathrm{XM}=0$.

$Y M=J * H P / 2$.

WRITE $(10, *) X M, Y M, 1 / \operatorname{SIGG}(0, J+M)$ 
DO $150 \mathrm{I}=1,(\mathrm{~N}-1), 2$

DO $151 \mathrm{~J}=1,(\mathrm{M}-1), 2$

$X M=(X G(I-1, J+M-1)+X G(I-1, J+M+1)+X G(I+1, J+M-1)+X G(I+1, J+M+1)) / 4$.

$Y M=(Y G(I-1, J+M-1)+Y G(I-1, J+M+1)+Y G(I+1, J+M-1)+Y G(I+1, J+M+1)) / 4$.

C

...Cálculo do campo elétrico sobre uma linha de campo no topo da região de ...Poisson.

$\mathrm{FI}=1$.

$\mathrm{EFI}=\mathrm{ABS}(\operatorname{APSIG}(2 * \mathrm{M}-7) * \operatorname{SIGG}(0,2 * \mathrm{M}-7))$

WRITE(50,*) FI, EFI

DO $300 \mathrm{I}=1,(\mathrm{~N}-1), 2$

$\mathrm{FI}=1 .-\mathrm{I} * \mathrm{H} / 2$.

$\mathrm{XPSI}=(\mathrm{XG}(\mathrm{I}+1,2 * \mathrm{M}-6)+\mathrm{XG}(\mathrm{I}-1,2 * \mathrm{M}-6)-\mathrm{XG}(1+1,2 * \mathrm{M}-8)-$

$1 \mathrm{XG}(\mathrm{I}-1,2 * \mathrm{M}-8)) /(2, * \mathrm{HP})$

$\mathrm{XPSI} 2=\mathrm{XPSI} * \mathrm{XPSI}$

$Y P S I=(Y G(I+1,2 * M-6)+Y G(I-1,2 * M-6)-Y G(I+1,2 * M-8)-$

1 $\mathrm{YG}(\mathrm{I}-1,2 * \mathrm{M}-8)) /(2 . * \mathrm{HP})$

YPSI $=$ YPSI $*$ YPSI

$\mathrm{AP}=\mathrm{ABS}(\mathrm{APSIG}(2 * \mathrm{M}-7))$

$\mathrm{EFI}=\mathrm{AP} * \operatorname{SIGG}(1,2 * \mathrm{M}-7) *(\mathrm{XPSI} 2+\mathrm{YPSI}) * *(-1 . / 2$.

300 CONTINUE

WRITE(50,*) FI, EFI

$\mathrm{FI}=1$.

$\mathrm{EXAT}=\mathrm{E} 0$

WRITE $(60, *) \mathrm{Fl}$, EXAT

DO $400 \mathrm{I}=1,(\mathrm{~N}-1), 2$

$\mathrm{FI}=1 .-\mathrm{I} * \mathrm{H} / 2$.

$\mathrm{X}=\mathrm{C} 1 \mathrm{~S} *((\mathrm{C} 2 \mathrm{~S} *(1 .-\mathrm{FI})+1) * *.(2 . / 3)-1$.

EXAT $=\mathrm{E} 0 *(1 .+(2 . * \mathrm{RHO0} / \mathrm{E} 0) * \mathrm{X}) * *(1 . / 2$.

400

WRITE $(60, *)$ FI, EXAT

CONTINUE

$\mathrm{FI}=1$.

$\mathrm{XFI}=-(\mathrm{XG}(2,2 * \mathrm{M}-6)+\mathrm{XG}(2,2 * \mathrm{M}-8)-\mathrm{XG}(0,2 * \mathrm{M}-6)-$

$1 \mathrm{XG}(0,2 * \mathrm{M}-8)) /(2 . * \mathrm{H})$

$\mathrm{XFI} 2=\mathrm{XFI} * \mathrm{XFI}$

$\mathrm{YFI}=-(\mathrm{YG}(2,2 * \mathrm{M}-6)+\mathrm{YG}(2,2 * \mathrm{M}-8)-\mathrm{YG}(0,2 * \mathrm{M}-6)-$

1 $\mathrm{YG}(0,2 * \mathrm{M}-8)) /(2 * * \mathrm{H})$

YFI2 $=$ YFI*YFI

$\mathrm{EFI1}=(\mathrm{XFI} 2+\mathrm{YFI}) * *(-1 . / 2$.

WRITE $(70, *)$ FI, EFI1

DO $500 \mathrm{I}=1,(\mathrm{~N}-1), 2$

$\mathrm{FI}=1 .-\mathrm{I} * \mathrm{H} / 2$.

$\mathrm{XFI}=-(\mathrm{XG}(\mathrm{I}+1,2 * \mathrm{M}-6)+\mathrm{XG}(\mathrm{I}+1,2 * \mathrm{M}-8)-\mathrm{XG}(\mathrm{I}-1,2 * \mathrm{M}-6)-$

$1 \mathrm{XG}(\mathrm{I}-1,2 * \mathrm{M}-8)) /(2 * \mathrm{H})$

$\mathrm{XFI}=\mathrm{XFI} * \mathrm{XFI}$

$\mathrm{YFI}=-(\mathrm{YG}(\mathrm{I}+1,2 * \mathrm{M}-6)+\mathrm{YG}(\mathrm{I}+1,2 * \mathrm{M}-8)-\mathrm{YG}(\mathrm{I}-1,2 * \mathrm{M}-6)-$ 1

$\mathrm{YFI} 2=\mathrm{YFI}$ YFI

$E F I 1=(X F I 2+Y F I 2) * *(-1 . / 2$.

WRITE(70,*) FI, EFI1

$\mathrm{C}$ CONTINUE

...Cálculo e resultados para a condição de ortogonalidade entre linhas de ...campo e curvas equipotenciais.

DO $161 \mathrm{~J}=2,(2 * \mathrm{M}-2), 2$

DO $162 \mathrm{I}=2,(\mathrm{~N}-2), 2$

$\begin{array}{ll}\mathrm{C} & \mathrm{XFI}=-(\mathrm{XG}(\mathrm{I}+2, \mathrm{~J})-\mathrm{XG}(\mathrm{I}-2, \mathrm{~J})) /(2, * \mathrm{H}) \\ \mathrm{C} & \mathrm{XPSI}=(\mathrm{XG}(\mathrm{I}, \mathrm{J}+2)-\mathrm{XG}(\mathrm{I}, \mathrm{J}-2)) /(2 . * \mathrm{HP})\end{array}$ 


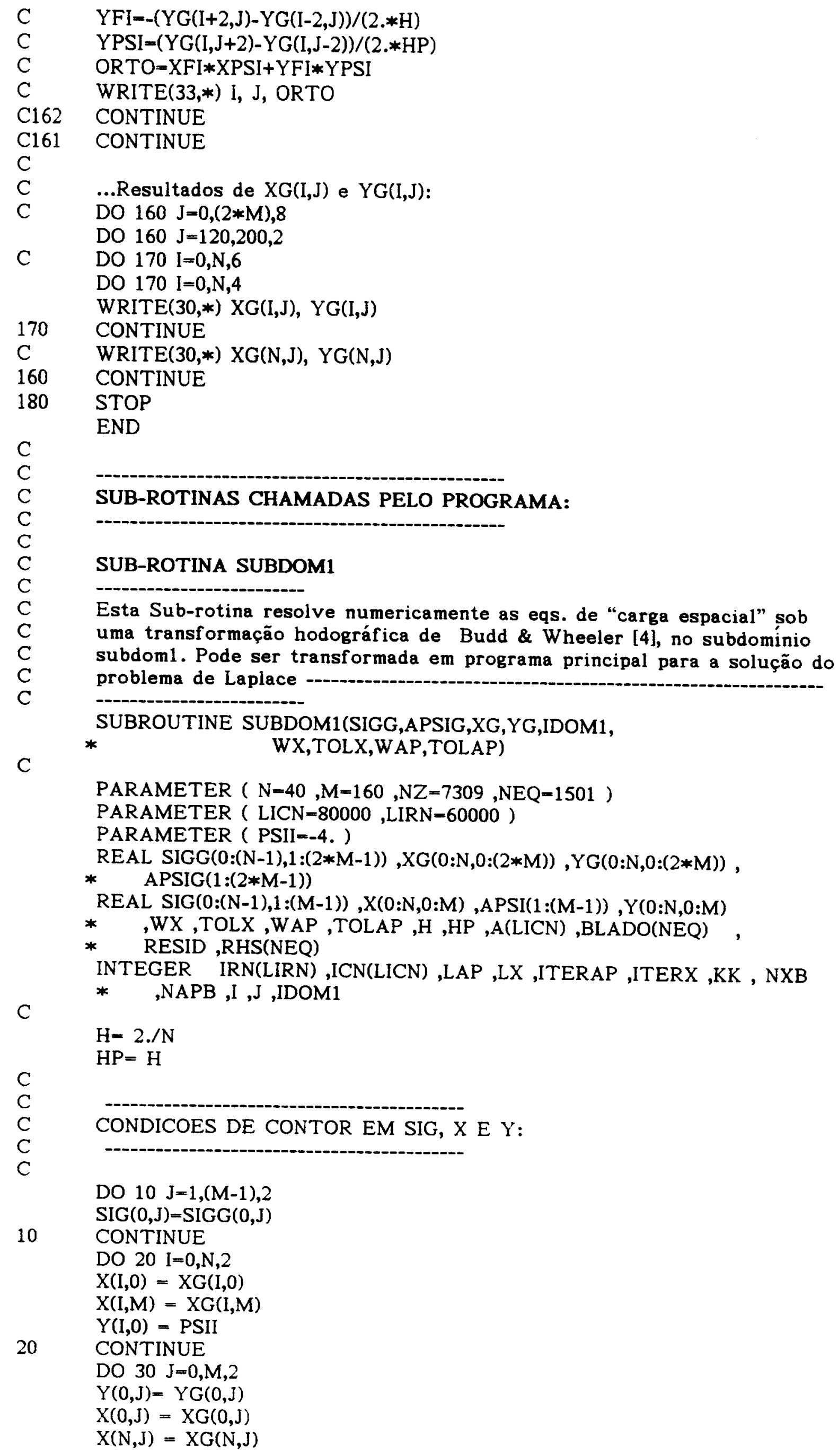


FUNÇÕES TENTETIVA PARA SIG, X E APSI:

DO $40 \mathrm{~J}=1,(\mathrm{M}-1), 2$

$\operatorname{APSI}(J)=\operatorname{APSIG}(J)$

DO $50 \mathrm{I}=1,(\mathrm{~N}-1), 2$

$\operatorname{SIG}(\mathrm{I}, \mathrm{J})=\operatorname{SIGG}(\mathrm{I}, \mathrm{J})$

C

...A função, tentativa para SIGG(I,J) no SUBDOM1 coincide com a solução

$\mathrm{C}$ ...correta. E tal que sempre SIGG(I,J)=1. (Ela corresponde a uma

C ...distribuição de carga espacial nula).

50 CONTINUE

40 CONTINUE

DO $60 \mathrm{~J}=2,(\mathrm{M}-2), 2$

DO $70 \mathrm{I}=2,(\mathrm{~N}-2), 2$

70

$\mathrm{X}(\mathrm{I}, \mathrm{J})=\mathrm{XG}(\mathrm{I}, \mathrm{J})$

CONTINUE

60 CONTINUE

$\mathrm{C}$

ITERAP $=0$

ITERAP $=$ ITERAP+1

ITERX $=0$

$90 \quad$ ITERX $=$ ITERX +1

CALL SUBGAN(X,SIG,APSI,A,BLADO,IRN,ICN,LIRN,LICN,N,M,H,HP)

TYPE *,

TYPE *,'----RESOLVENDO O SISTEMA DE EQS. LINEARES----'

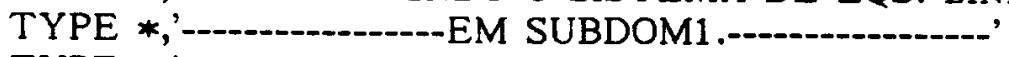

TYPE *,

CALL SUBSOL(A,IRN,ICN,N,M,LIRN,LICN,BLADO,NEQ,NZ)

$\mathrm{KK}=0$

$\mathrm{NXB}=0$

C

...Cálculo iterativo de X.

TYPE *,'*******************************************'

TYPE *,'CALCULANDO X(I,J) EM SUBDOM1,***** ITERX -',ITERX

TYPE *, '********************************************

DO $100 \mathrm{~J}=2,(\mathrm{M}-2), 2$

DO $110 \mathrm{I}=2,(\mathrm{~N}-2), 2$

$\mathrm{KK}=\mathrm{KK}+1$

$\mathrm{XNOVO}=\mathrm{WX} * \mathrm{X}(\mathrm{I}, \mathrm{J})+(1 .-\mathrm{WX}) * \mathrm{BLADO}(\mathrm{KK})$

DELX $=A B S(X N O V O-X(I, J))$

IF(DELX.LE.TOLX) NXB $=\mathrm{NXB}+1$

110

$\mathrm{X}(\mathrm{I}, \mathrm{J})=\mathrm{XNOVO}$

100 CONTINUE

APROVX $=(\operatorname{REAL}(\mathrm{NXB}) /((\mathrm{N} / 2-1) *(\mathrm{M} / 2-1))) * 100$.

IF(ITERX.LT.15) THEN

IF(APROVX.LT.100.) THEN

GO TO 90

ENDIF

$\mathrm{NAPB}=0$

GO TO 119

ENDIF

IF(APROVX.LT .100.) THEN

TYPE '(A)','S NAO HA CONVERGENCIA PARA X EM SUBDOM1.'

TYPE '(A)','S TENTE NOVOS PESOS E TOLERANCIAS'

IDOM $1=1$

GO TO 210

ENDIF

$\mathrm{NAPB}=0$

C ...Cálculo iterativo de APSI.

119 TYPE *,@@@@@@@@@@@@@@@@@@@@@@@@@@@@@@@@@@@@@@@@@ 
TYPE *',CALCULANDO APSI(J) EM SUBDOM1.*****ITERAP $\approx$ ',ITERAP TYPE *,'@@@@@@@@@@@@@@@@@@@@@@@@@@@@@@@@@@@@@@@@@' DO $120 \mathrm{~J}=1,(\mathrm{M}-1), 2$

$\mathrm{XFI}=-(4 . *(\mathrm{X}(2, \mathrm{~J}+1)+\mathrm{X}(2, \mathrm{~J}-1))-3 . *(\mathrm{X}(0, \mathrm{~J}-1)+\mathrm{X}(0, \mathrm{~J}+1))-\mathrm{X}(4, \mathrm{~J}+1)-$

* $\mathrm{X}(4, \mathrm{~J}-1)) /(4, * \mathrm{H})$

APAT $=1 . /(\operatorname{SIG}(0, \mathrm{~J}) * \mathrm{XFI})$

APNOVO $=$ WAP $* A P S I(J)+(1 .-$ WAP $) * A P A T$

DELAP $=$ ABS(APNOVO-APSI $(J))$

IF(DELAP.LE.TOLAP) NAPB - NAPB+1

120

$\operatorname{APSI}(\mathrm{J})=$ APNOVO

CONTINUE

$A P R O A P=(\operatorname{REAL}(N A P B) /(\operatorname{REAL}(M / 2))) * 100$

IF(ITERAP.LT.15) THEN

IF(APROAP.LT.100.) THEN

GO TO 80

ENDIF

GO TO 139

ENDIF

IF(APROAP.LT.100.) THEN

TYPE '(A)','\$ NAO HA CONVERGENCIA PARA APSI EM SUBDOM1.'

TYPE '(A)','\$ TENTE NOVOS PESOS E TOLERANCIAS'

IDOM $1=1$

GO TO 210

ENDIF

139 DO $140 \mathrm{~J}=2,(\mathrm{M}-2), 2$

DO $150 \mathrm{I}=2,(\mathrm{~N}-2), 2$

ASIG $=(\operatorname{APSI}(\mathrm{J}+1) * \operatorname{SIG}(\mathrm{I}-1, \mathrm{~J}+1)+\operatorname{APSI}(\mathrm{J}-1) * \operatorname{SIG}(\mathrm{I}-1, \mathrm{~J}-1)) / 2$.

$\mathrm{XPSI}=(\mathrm{X}(\mathrm{I}, \mathrm{J}+2)+\mathrm{X}(\mathrm{I}-2, \mathrm{~J}+2)-\mathrm{X}(\mathrm{I}, \mathrm{J}-2)-\mathrm{X}(\mathrm{I}-2, \mathrm{~J}-2)) /(4 . * \mathrm{HP})$

$\mathrm{Y}(\mathrm{I}, \mathrm{J})=\mathrm{Y}(\mathrm{I}-2, \mathrm{~J})+\mathrm{H} * \mathrm{XPSI} / \mathrm{ASIG}$

150

CONTINUE

$\mathrm{Y}(\mathrm{N}, \mathrm{J})-\mathrm{Y}((\mathrm{N}-2), \mathrm{J})$

140 CONTINUE

C

C

...Conversão dos resultados de APSI(J) para APSIG(J):

DO $160 \mathrm{~J}=1,(\mathrm{M}-1), 2$

$\operatorname{APSIG}(\mathrm{J})=\operatorname{APSI}(\mathrm{J})$

CONTINUE

DO $170 \mathrm{I}=1,(\mathrm{~N}-1), 2$

DO $180 \mathrm{~J}=1,(\mathrm{M}-1), 2$

$\operatorname{SIGG}(\mathrm{I}, \mathrm{J})=\operatorname{SIG}(\mathrm{I}, \mathrm{J})$

180

CONTINUE

170 CONTINUE

C

C ...Conversão dos resultados de $X(I, J)$ e $Y(I, J)$ para os

C ...correspondentes XG(I,J) e YG(I,J):

DO $190 \mathrm{~J}=2,(\mathrm{M}-2), 2$

DO $200 \mathrm{I}=2,(\mathrm{~N}-2), 2$

$X \mathrm{G}(\mathrm{I}, \mathrm{J})=\mathrm{X}(\mathrm{I}, \mathrm{J})$

$Y G(I, J)=Y(I, J)$

200

CONTINUE

$Y G(N, J)=Y(N, J)$

190 CONTINUE

210 RETURN

END

C

\section{SUB-ROTINA SUBDOM2}

Esta Sub-rotina resolve numericamente as eqs. de carga espacial sob uma transformação hodográfica de Budd \& Wheeler [4], no subdomínio subdom2. Pode ser transformada em programa principal para tratar o problema de carga espacial ocupando totalmente um dominio simplesmente 
C

conexo.

C

C

$\mathrm{C}$

SUBROUTINE SUBDOM2(SIGG,APSIG,XG,YG,IDOM2,

*

WSIG, TOLSIG, WX, TOLX, WAP,TOLAP)

PARAMETER ( $\mathrm{N}=40, \mathrm{M}=160, \mathrm{NZ}=7309, \mathrm{NEQ}=1501)$

PARAMETER ( $\mathrm{LICN}=80000$, LIRN $=60000)$

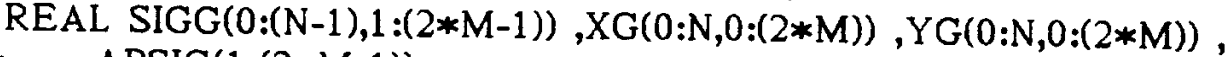

* $\quad \operatorname{APSIG}(1:(2 * \mathrm{M}-1))$

REAL SIG(0:(N-1),1:(M-1)),X(0:N,0:M),APSI(1:(M-1)), Y(0:N,0:M)

* ,WSIG ,TOLSIG ,WX ,TOLX ,WAP ,TOLAP ,H , HP ,A(LICN)

* ,BLADO(NEQ) ,RESID ,RHS(NEQ)

INTEGER IRN(LIRN), ICN(LICN), LAP ,LX ,ITERAP ,ITERX ,KK ,NXB

C

$\mathrm{H}=2 . / \mathrm{N}$

$\mathrm{HP}=\mathrm{H}$

C

C

$\mathrm{C}$

10

CONDICOES DE CONTOR EM SIG, X E Y:

DO $10 \mathrm{~J}=1,(\mathrm{M}-1), 2$

$\operatorname{SIG}(0, J)=\operatorname{SIGG}(0, J+M)$

CONTINUE

DO $20 \mathrm{I}=0, \mathrm{~N}, 2$

$X(I, 0)=X G(I, M)$

20

$X(I, M)=X G(1,2 * M)$

CONTINUE

DO $30 \mathrm{~J}=0, \mathrm{M}, 2$

$Y(0, J)=Y G(0, J+M)$

$X(0, J)=X G(0, J+M)$

$X(N, J)=X G(N, J+M)$

30

CONTINUE

C

C

C

C

FUNÇÕES TENTATIVA PARA SIG, X E APSI:

C

DO $40 \mathrm{~J}=1,(\mathrm{M}-1), 2$

$\operatorname{APSI}(\mathrm{J})=\operatorname{APSIG}(\mathrm{J}+\mathrm{M})$

DO $50 \mathrm{I}=1,(\mathrm{~N}-1), 2$

$\operatorname{SIG}(I, J)=\operatorname{SIGG}(\mathrm{I}, \mathrm{J}+\mathrm{M})$

50

CONTINUE

40 CONTINUE

DO $60 \mathrm{~J}=2,(\mathrm{M}-2), 2$

DO $70 \mathrm{I}=2,(\mathrm{~N}-2), 2$

$X(I, J)=X G(I, J+M)$

70 CONTINUE

60 CONTINUE

C

ITERAP $=0$

80 ITERAP $=$ ITERAP+1

ITERX $=0$

90 ITERX $=$ ITERX +1

INDSIG $=0$

CALL SUBSIG(SIG,X,APSI,N,M,H,HP,WSIG,TOLSIG,INDSIG)

IF(INDSIG.EQ.1) THEN

IDOM $2=1$

GO TO 210

ENDIF

CALL SUBGAN(X,SIG,APSI,A,BLADO,IRN,ICN,LIRN,LICN,N,M,H,HP)

TYPE *, 
TYPE *,'----RESOLVENDO O SISTEMA DE EQS. LINEARES-..-'

TYPE *

TYPE *

CALL SUBSOL(A,IRN,ICN,N,M,LIRN,LICN,BLADO,NEQ,NZ)

$\mathrm{KK}=0$

$\mathrm{NXB}=0$

...Cálculo iterativo de X.

TYPE

TYPE *',CALCULANDO X(I,J) EM SUBDOM2.***** ITERX =',ITERX

DO $100 \mathrm{~J}=2,(\mathrm{M}-2), 2$

DO $110 \mathrm{I}=2,(\mathrm{~N}-2), 2$

$\mathrm{KK}=\mathrm{KK}+1$

$\mathrm{XNOVO}=\mathrm{WX} * \mathrm{X}(\mathrm{I}, \mathrm{J})+(1 .-\mathrm{WX}) * \mathrm{BLADO}(\mathrm{KK})$

$D E L X=A B S(X N O V O-X(I, J))$

IF(DELX.LE.TOLX) NXB= NXB+1

110

$\mathrm{X}(\mathrm{I}, \mathrm{J})=\mathrm{XNOVO}$

100 CONTINUE

APROVX $=(\operatorname{REAL}(\mathrm{NXB}) /((\mathrm{N} / 2-1) *(\mathrm{M} / 2-1))) * 100$.

IF(ITERX.LT.12) THEN

IF(APROVX.LT.100.) THEN

GO TO 90

ENDIF

$\mathrm{NAPB}=0$

TYPE *,'@@@@@@@@@@@@@@@@@@@@@@@@@@@@@@@@@@@@@@@@@'

TYPE *'CALCULANDO APSI(J) EM SUBDOM2.***** ITERAP-',ITERAP

TYPE *,'@@@@@@@@@@@@@@@@@@@@@@@@@@@@@@@@@@@@@@@@@' GO TO 119

ENDIF

IF(APROVX.LT.100.) THEN

TYPE '(A)','S NAO HA CONVERGENCIA PARA X EM SUBDOM2.'

TYPE '(A)','S TENTE NOVOS PESOS E TOLERANCIAS'

IDOM2 $=1$

GO TO 210

ENDIF

$\mathrm{NAPB}=0$

C ...Cálculo iterativo de APSI.

TYPE *,'@@@@@@@@@@@@@@@@@@@@@@@@@@@@@@@@@@@@@@@@@'

TYPE $*$,'CALCULANDO APSI(J) EM SUBDOM2.*****ITERAP=',ITERAP

119 DO $120 \mathrm{~J}=1,(\mathrm{M}-1), 2 \mathrm{~T}$,

$\mathrm{XFI}=-(4 \cdot *(\mathrm{X}(2, \mathrm{~J}+1)+\mathrm{X}(2, \mathrm{~J}-1))-3 . *(\mathrm{X}(0, \mathrm{~J}-1)+\mathrm{X}(0, \mathrm{~J}+1))-\mathrm{X}(4, \mathrm{~J}+1)-$

* $\mathrm{X}(4, \mathrm{~J}-1)) /(4 . * \mathrm{H})$

APAT $=1 . /(\mathrm{SIG}(0, \mathrm{~J}) * \mathrm{XFI})$

APNOVO $=$ WAP $* A P S I(J)+(1 .-W A P) * A P A T$

DELAP = ABS(APNOVO-APSI(J))

IF(DELAP.LE.TOLAP) NAPB $=$ NAPB +1

APSI $(J)=$ APNOVO

120 CONTINUE

APROAP $=(\operatorname{REAL}(\mathrm{NAPB}) /(\operatorname{REAL}(M / 2))) * 100$.

IF(ITERAP.LT.15) THEN

IF(APROAP.LT.100.) THEN

GO TO 80

ENDIF

GO TO 139

ENDIF

IF(APROAP.LT.100.) THEN

TYPE '(A)','S NAO HA CONVERGENCIA PARA APSI EM SUBDOM2.'

TYPE '(A)','S TENTE NOVOS PESOS E TOLERANCIAS'

IDOM $2=1$

GO TO 210

ENDIF 
C ...Cálculo de Y.

139 DO $140 \mathrm{~J}=2,(\mathrm{M}-2), 2$

DO $150 \mathrm{I}=2,(\mathrm{~N}-2), 2$

$A S I G=(\operatorname{APSI}(J+1) * \operatorname{SIG}(I-1, J+1)+\operatorname{APSI}(J-1) * \operatorname{SIG}(I-1, J-1)) / 2$.

$\mathrm{XPSI}=(\mathrm{X}(\mathrm{I}, \mathrm{J}+2)+\mathrm{X}(\mathrm{I}-2, \mathrm{~J}+2)-\mathrm{X}(\mathrm{I}, \mathrm{J}-2)-\mathrm{X}(\mathrm{I}-2, \mathrm{~J}-2)) /(4 . * \mathrm{HP})$

$Y(I, J)=Y(I-2, J)+H * X P S I / A S I G$

150 CONTINUE

$\mathrm{Y}(\mathrm{N}, \mathrm{J})=\mathrm{Y}((\mathrm{N}-2), \mathrm{J})$

140 CONTINUE

C

C

..Conversão dos resultados de $\operatorname{SIG}(J)$ e APSI(J) para

...SIGG(I,J) e APSIG(J):

DO $160 \mathrm{~J}=1,(\mathrm{M}-1), 2$

$\operatorname{APSIG}(\mathrm{J}+\mathrm{M})=\operatorname{APSI}(\mathrm{J})$

CONTINUE

DO $170 \mathrm{I}=1,(\mathrm{~N}-1), 2$

DO $180 \mathrm{~J}=1,(\mathrm{M}-1), 2$

180

$\operatorname{SIGG}(\mathrm{I}, \mathrm{J}+\mathrm{M})=\operatorname{SIG}(\mathrm{I}, \mathrm{J})$

CONTINUE

C

C

C

200 CONTINUE

...Conversão dos resultados de $\mathrm{X}(\mathrm{I}, \mathrm{J})$ e $\mathrm{Y}(\mathrm{I}, \mathrm{J})$ para os ...correspondentes XG(I,J) e YG(I,J):

DO $190 \mathrm{~J}=2,(\mathrm{M}-2), 2$

DO $200 \mathrm{I}=2,(\mathrm{~N}-2), 2$

$X G(I, J+M)=X(I, J)$

$Y G(I, J+M)=Y(I, J)$

$\mathrm{YG}(\mathrm{N}, \mathrm{J}+\mathrm{M})=\mathrm{Y}(\mathrm{N}, \mathrm{J})$

190 CONTINUE

210 RETURN

END

C

C SUB-ROTINA PARA CALCULAR SIGMA

C

SUBROUTINE SUBSIG(SIG,X,APSI,N,M,H,HP,WSIG, TOLSIG,INDSIG)

REAL SIG(0:(N-1),1:(M-1)),X(0:N,0:M),APSI $(1:(M-1)), H$, WSIG ,

* TOLSIG, HP

INTEGER $\mathrm{N}, \mathrm{M}, \mathrm{I}, \mathrm{J}, \mathrm{LSIG}$, ,INDSIG

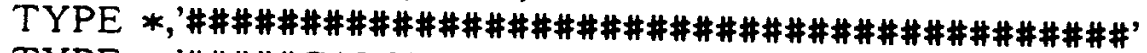

TYPE *,\#\#\#\#\#CALCULANDO SIG(I,J) EM SUBDOM2.\#\#\#\#\#'

TYPE $*$,

DO $10 \mathrm{~J}=1,(\mathrm{M}-1), 2$

DO $20 \mathrm{I}=1,(\mathrm{~N}-1), 2$

$30 \quad \mathrm{LSIG}=0$

IF(I.EQ.1) THEN

$\operatorname{SIGV}=\operatorname{SIG}(\mathrm{I}, \mathrm{J})$

C ...Nos nossos cálculos DELPSI $=\mathrm{H}$ e DELFI $=-\mathrm{H}$.

$\mathrm{XF}=\mathrm{X}(2, \mathrm{~J}+1)+\mathrm{X}(2, \mathrm{~J}-1)-\mathrm{X}(0, \mathrm{~J}+1)-\mathrm{X}(0, \mathrm{~J}-1)$

$\mathrm{XF} 2=\mathrm{XF} * \mathrm{XF}$

$X P=X(2, J+1)+X(0, J+1)-X(2, J-1)-X(0, J-1)$

$\mathrm{XP} 2=\mathrm{XP} * \mathrm{XP}$

$\operatorname{ASIG} 2=\operatorname{APSI}(\mathrm{J}) * \operatorname{APSI}(\mathrm{J}) * \operatorname{SIGV} * \operatorname{SIGV}$

$\operatorname{SIG}(\mathrm{I}, \mathrm{J})=\operatorname{SIG}(0, \mathrm{~J})+\mathrm{XF} 2 /(8 . * \mathrm{H})+(\mathrm{XP} 2 * \mathrm{H}) /(8 . * \mathrm{ASIG} 2 * \mathrm{HP} * \mathrm{HP})$

$\operatorname{SIG}(\mathrm{I}, \mathrm{J})=$ WSIG $* \operatorname{SIGV}+(1 .-\mathrm{WSIG}) * \operatorname{SIG}(\mathrm{I}, \mathrm{J})$

DELSIG $=$ ABS(SIG(I,J)-SIGV)

IF(DELSIG.GT.TOLSIG) THEN

IF(LSIG.EQ.25) THEN

TYPE '(A)','S NUMERO DE ITERACOES DE SIGMA=12'

TYPE '(A)','S NAO HA CONVERGENCIA PARA SIGG EM SUBDOM2.

TYPE '(A)','STENTE NOVOS PESOS E TOLERANCIAS'

INDSIG $=1$ 
GO TO 21

ENDIF

GO TO 30

ENDIF

GO TO 20

ENDIF

SIGV1 $=\operatorname{SIG}(\mathrm{I}, \mathrm{J})$

SIGV2 $=\operatorname{SIG}(\mathrm{I}-2, \mathrm{~J})$

$\mathrm{XF} 1=\mathrm{X}(\mathrm{I}+1, \mathrm{~J}-1)+\mathrm{X}(\mathrm{I}+1, \mathrm{~J}+1)-\mathrm{X}(\mathrm{I}-1, \mathrm{~J}-1)-\mathrm{X}(\mathrm{I}-1, \mathrm{~J}+1)$

$\mathrm{XF} 12=\mathrm{XF} 1 * \mathrm{XF} 1$

$\mathrm{XF} 2=\mathrm{X}(\mathrm{I}-1, \mathrm{~J}-1)+\mathrm{X}(\mathrm{I}-1, \mathrm{~J}+1)-\mathrm{X}(\mathrm{I}-3, \mathrm{~J}-1)-\mathrm{X}(\mathrm{I}-3, \mathrm{~J}+1)$

$\mathrm{XF} 22=\mathrm{XF} 2 * \mathrm{XF} 2$

$\mathrm{XP1}=\mathrm{X}(\mathrm{I}+1, \mathrm{~J}+1)+\mathrm{X}(\mathrm{I}-1, \mathrm{~J}+1)-\mathrm{X}(\mathrm{I}+1, \mathrm{~J}-1)-\mathrm{X}(\mathrm{I}-1, \mathrm{~J}-1)$

$\mathrm{XP1} 2=\mathrm{XP} 1 * \mathrm{XP} 1$

ASIG $1=\operatorname{APSI}(\mathrm{J}) * \operatorname{APSI}(\mathrm{J}) * \operatorname{SIGV} 1 * \operatorname{SIGV} 1$

$X P 2=X(I-1, J+1)+X(I-3, J+1)-X(I-1, J-1)-X(I-3, J-1)$

$\mathrm{XP} 22=\mathrm{XP} 2 * \mathrm{XP} 2$

ASIG $2=\operatorname{APSI}(\mathrm{J}) * \operatorname{APSI}(\mathrm{J}) * \operatorname{SIGV} 2 * \operatorname{SIGV} 2$

$\operatorname{SIG}(I, J)=\operatorname{SIG}(\mathrm{I}-2, \mathrm{~J})+(\mathrm{XF} 12+\mathrm{XF} 22) /(8 . * \mathrm{H})+(\mathrm{XP} 12 * \mathrm{H}) /(8 . * \mathrm{ASIG} 1 * \mathrm{HP} * \mathrm{HP})$

* $+(\mathrm{XP} 22 * \mathrm{H}) /(8 . * A S I G 2 * H P * H P)$

$\operatorname{SIG}(I, J)=$ WSIG $* \operatorname{SIGV} 1+(1 .-$ WSIG $) * \operatorname{SIG}(I, J)$

DELSIG = ABS(SIG(I,J)-SIGV1)

IF(DELSIG.GT.TOLSIG) THEN

IF(LSIG.EQ.25) THEN

TYPE '(A)','\$ NUMERO DE ITERACOES DE SIGMA=12'

TYPE '(A)','S NAO HA CONVERGENCIA PARA SIGG EM SUBDOM2.' INDSIG $=1$

GO TO 21

ENDIF

GO TO 30

ENDIF

CONTINUE

CONTINUE

RETURN

END

C
C
C
C
C
C
C
C
C

\section{SUB-ROTINA SUBGAN}

Ordena os elementos não-nulos da matriz diagonal esparsa, $M$, e constroi o vetor $\mathrm{BLADO} \equiv \mathbb{B}$, eq. matricial $\mathbb{M X}=\mathbb{B}$. Em outras palavras, constroi os arranjos ordenados $A(L I C N)$, IRN(LIRN), ICN(LINC) e

$\mathbb{R H S}(\mathbb{N}) \equiv$ BLADO(N) para futuras chamadas da biblioteca NAG.

SUBROUTINE SUBGAN(X,SIG,APSI,AP,BLADO,IRN,ICN,LIRN,LICN,N,M,H,HP)

REAL X(0:N,0:M) ,SIG(0:(N-1),1:(M-1)), APSI(1:(M-1)),AP(LICN),

* BLADO(1:(N/2-1)*(M/2-1)),H, HP

INTEGER IRN(LIRN), ICN(LICN), LIRN ,LICN , N , M , K , IR ,IC , L ,

C

... Construce

$\dddot{\mathrm{K}}=0$

$\mathrm{IR}=0$

$\mathrm{IC}=(\mathrm{N} / 2-1)$

DO $10 \mathrm{~J}=2,(\mathrm{M}-4), 2$

DO $20 \mathrm{I}=2,(\mathrm{~N}-2), 2$

$\mathrm{K}=\mathrm{K}+1$

$\mathrm{IR}=\mathrm{IR}+1$

$\mathrm{IC}=\mathrm{IC}+1$

$\operatorname{ALF} 1=2 . /(\operatorname{APSI}(J+1) *(\operatorname{SIG}(\mathrm{I}-1, \mathrm{~J}+1)+\operatorname{SIG}(\mathrm{I}+1, \mathrm{~J}+1)))$

$A P(K)=A L F 1$

$I R N(K)=I R$

$\mathrm{ICN}(\mathrm{K})=\mathrm{IC}$ 
20 CONTINUE

10 CONTINUE

$I R=N / 2-1$

$\mathrm{IC}=0$

DO $30 \mathrm{~J}=4,(\mathrm{M}-2), 2$

DO $40 \mathrm{I}=2,(\mathrm{~N}-2), 2$

$\mathrm{K}=\mathrm{K}+1$

$\mathrm{IR}=\mathrm{IR}+1$

$\mathrm{IC}=\mathrm{IC}+1$

$\mathrm{ALF} 3=2 . /(\mathrm{APSI}(\mathrm{J}-1) *(\operatorname{SIG}(\mathrm{I}-1, \mathrm{~J}-1)+\mathrm{SIG}(\mathrm{I}+1, \mathrm{~J}-1)))$

$A P(K)=A L F 3$

$\operatorname{IRN}(K)=\operatorname{IR}$

$I C N(K)=I C$

40 CONTINUE

30 CONTINUE

$\mathrm{IR}=0$

$\mathrm{IC}=0$

DO $50 \mathrm{~J}=2,(\mathrm{M}-2), 2$

DO $60 \mathrm{I}=2,(\mathrm{~N}-2), 2$

$\mathrm{K}=\mathrm{K}+1$

IR $=$ IR +1

$\mathrm{IC}=\mathrm{IC}+1$

ALF1 $-2 . /(\operatorname{APSI}(\mathrm{J}+1) *(\operatorname{SIG}(\mathrm{I}-1, \mathrm{~J}+1)+\operatorname{SIG}(\mathrm{I}+1, \mathrm{~J}+1)))$

$\operatorname{ALF} 2=(\operatorname{APSI}(\mathrm{J}-1) * \operatorname{SIG}(\mathrm{I}+1, \mathrm{~J}-1)+\operatorname{APSI}(\mathrm{J}+1) * \operatorname{SIG}(\mathrm{I}+1, \mathrm{~J}+1)) / 2$.

$\mathrm{ALF} 2=(\mathrm{HP} / \mathrm{H}) *(\mathrm{HP} / \mathrm{H}) * \mathrm{ALF} 2$

ALF $3=2 . /($ APSI $(\mathrm{J}-1) *(\operatorname{SIG}(\mathrm{I}-1, \mathrm{~J}-1)+\mathrm{SIG}(\mathrm{I}+1, \mathrm{~J}-1)))$

$\operatorname{ALF} 4=(\operatorname{APSI}(\mathrm{J}-1) * \operatorname{SIG}(\mathrm{I}-1, \mathrm{~J}-1)+\operatorname{APSI}(\mathrm{J}+1) * \operatorname{SIG}(\mathrm{I}-1, \mathrm{~J}+1)) / 2$.

$\mathrm{ALF} 4=(\mathrm{HP} / \mathrm{H}) *(\mathrm{HP} / \mathrm{H}) * \mathrm{ALF} 4$

$A L F=-(A L F 1+A L F 2+A L F 3+A L F 4)$

$A P(K)=A L F$

$I R N(K)=I R$

$\mathrm{ICN}(\mathrm{K})=\mathrm{IC}$

60 CONTINUE

50 CONTINUE

DO $70 \mathrm{~L}=1,(\mathrm{M} / 2-1)$

$\mathrm{IR}=(\mathrm{L}-1) *(\mathrm{~N} / 2-1)$

$\mathrm{IC}=(\mathrm{L}-1) *(\mathrm{~N} / 2-1)+1$

DO $80[=2,(\mathrm{~N}-4), 2$

$\mathrm{K}=\mathrm{K}+1$

$\mathrm{IR}=\mathrm{IR}+1$

$\mathrm{IC}=\mathrm{IC}+1$

$\mathrm{ALF} 2=(\mathrm{APSI}(2 * \mathrm{~L}-1) * \operatorname{SIG}(1+1,2 * \mathrm{~L}-1)$

* $\quad+\mathrm{APSI}(2 * \mathrm{~L}+1) * \mathrm{SIG}(\mathrm{I}+1,2 * \mathrm{~L}+1)) / 2$.

$\mathrm{ALF} 2=(\mathrm{HP} / \mathrm{H}) *(\mathrm{HP} / \mathrm{H}) * \mathrm{ALF} 2$

$A P(K)=A L F 2$

$\operatorname{IRN}(K)=\mathrm{IR}$

$\mathrm{ICN}(\mathrm{K})=\mathrm{IC}$

80

CONTINUE

70 CONTINUE

DO $90 \mathrm{LL}=1,(\mathrm{M} / 2-1)$

$I R=(L L-1) *(N / 2-1)+1$

$\mathrm{IC}=(\mathrm{LL}-1) *(\mathrm{~N} / 2-1)$

DO $100 \mathrm{I}=4,(\mathrm{~N}-2), 2$

$\mathrm{K}=\mathrm{K}+1$

$\mathrm{IR}=\mathrm{IR}+1$

$\mathrm{IC}=\mathrm{IC}+1$

ALF $4=(\operatorname{APSI}(2 * \operatorname{LL}-1) * \operatorname{SIG}(I-1,2 * \mathrm{LL}-1)$

* $\quad+\mathrm{APSI}(2 * \mathrm{LL}+1) * \operatorname{SIG}(\mathrm{I}-1,2 * \mathrm{LL}+1)) / 2$.

$\mathrm{ALF} 4=(\mathrm{HP} / \mathrm{H}) *(\mathrm{HP} / \mathrm{H}) * \mathrm{ALF} 4$

$A P(K)=A L F 4$

$\operatorname{IRN}(\mathrm{K})=\operatorname{IR}$

$I C N(K)=I C$ 
C ...Construção de $\mathrm{BLADO} \equiv \mathbb{B}$.

$\mathrm{K}=0$

DO $110 \mathrm{~J}=2,(\mathrm{M}-2), 2$

IF(J.EQ.2) THEN

DO $120 \mathrm{I}=2,(\mathrm{~N}-2), 2$

$\mathrm{K}=\mathrm{K}+1$

IF(I.EQ.2) THEN

ALF $3=2 . /($ APSI $(J-1) *(\operatorname{SIG}(I-1, J-1)+\operatorname{SIG}(I+1, J-1)))$

$\operatorname{ALF} 4=(\operatorname{APSI}(\mathrm{J}-1) * \operatorname{SIG}(\mathrm{I}-1, \mathrm{~J}-1)+\operatorname{APSI}(\mathrm{J}+1) * \operatorname{SIG}(\mathrm{I}-1, \mathrm{~J}+1)) / 2$.

$\mathrm{ALF} 4=(\mathrm{HP} / \mathrm{H}) *(\mathrm{HP} / \mathrm{H}) * \mathrm{ALF} 4$

$\mathrm{BLADO}(\mathrm{K})=-\mathrm{ALF} 4 * \mathrm{X}(\mathrm{I}-2, \mathrm{~J})-\mathrm{ALF} 3 * \mathrm{X}(\mathrm{I}, \mathrm{J}-2)$

GO TO 120

ENDIF

IF(I.NE.(N-2)) THEN

ALF $3=2 . /(\operatorname{APSI}(\mathrm{J}-1) *(\operatorname{SIG}(\mathrm{I}-1, \mathrm{~J}-1)+\operatorname{SIG}(\mathrm{I}+1, \mathrm{~J}-1)))$

$\operatorname{BLADO}(\mathrm{K})=-\operatorname{ALF} 3 * \mathrm{X}(\mathrm{I}, \mathrm{J}-2)$

GO TO 120

ENDIF

$\operatorname{ALF} 2=(\operatorname{APSI}(\mathrm{J}-1) * \operatorname{SIG}(\mathrm{I}+1, \mathrm{~J}-1)+\operatorname{APSI}(\mathrm{J}+1) * \operatorname{SIG}(\mathrm{I}+1, \mathrm{~J}+1)) / 2$.

$\mathrm{ALF} 2=(\mathrm{HP} / \mathrm{H}) *(\mathrm{HP} / \mathrm{H}) * \mathrm{ALF} 2$

$\operatorname{ALF} 3=2 . /(\operatorname{APSI}(\mathrm{J}-1) *(\operatorname{SIG}(\mathrm{I}-1, \mathrm{~J}-1)+\operatorname{SIG}(\mathrm{I}+1, \mathrm{~J}-1)))$

120

$\operatorname{BLADO}(\mathrm{K})=-\mathrm{ALF} 3 * \mathrm{X}(\mathrm{I}, \mathrm{J}-2)-\mathrm{ALF} 2 * \mathrm{X}(\mathrm{I}+2, \mathrm{~J})$

CONTINUE

GO TO 110

ENDIF

IF(J.NE.(M-2)) THEN

DO $130 \mathrm{I}=2,(\mathrm{~N}-2), 2$

$\mathrm{K}=\mathrm{K}+1$

IF(I.EQ.2) THEN

ALF4 $=(\operatorname{APSI}(\mathrm{J}-1) * \operatorname{SIG}(\mathrm{I}-1, \mathrm{~J}-1)+\operatorname{APSI}(\mathrm{J}+1) * \operatorname{SIG}(\mathrm{I}-1, \mathrm{~J}+1)) / 2$.

$\mathrm{ALF} 4=(\mathrm{HP} / \mathrm{H}) *(\mathrm{HP} / \mathrm{H}) * \mathrm{ALF} 4$

$\operatorname{BLADO}(\mathrm{K})=-\mathrm{ALF} 4 * \mathrm{X}(\mathrm{I}-2, \mathrm{~J})$

GO TO 130

ENDIF

IF(I.NE.(N-2)) THEN

$\operatorname{BLADO}(\mathrm{K})=0$

GO TO 130

ENDIF

$\operatorname{ALF} 2=(\operatorname{APSI}(\mathrm{J}-1) * \operatorname{SIG}(I+1, \mathrm{~J}-1)+\operatorname{APSI}(\mathrm{J}+1) * \operatorname{SIG}(I+1, \mathrm{~J}+1)) / 2$.

$\mathrm{ALF} 2=(\mathrm{HP} / \mathrm{H}) *(\mathrm{HP} / \mathrm{H}) * \mathrm{ALF} 2$

130 CONTINUE

GO TO 110

ENDIF

DO $140 \mathrm{I}=2,(\mathrm{~N}-2), 2$

$\mathrm{K}=\mathrm{K}+1$

IF(I.EQ.2) THEN

$A L F 1=2 . /(\operatorname{APSI}(\mathrm{J}+1) *(\operatorname{SIG}(\mathrm{I}-1, \mathrm{~J}+1)+\operatorname{SIG}(\mathrm{I}+1, \mathrm{~J}+1)))$

$\operatorname{ALF} 4=(\operatorname{APSI}(\mathrm{J}-1) * \operatorname{SIG}(\mathrm{I}-1, \mathrm{~J}-1)+\operatorname{APSI}(\mathrm{J}+1) * \operatorname{SIG}(\mathrm{I}-1, \mathrm{~J}+1)) / 2$.

$\mathrm{ALF} 4=(\mathrm{HP} / \mathrm{H}) *(\mathrm{HP} / \mathrm{H}) * \mathrm{ALF} 4$

$\operatorname{BLADO}(\mathrm{K})=-\mathrm{ALF} 4 * \mathrm{X}(\mathrm{I}-2, \mathrm{~J})-\mathrm{ALF} 1 * \mathrm{X}(\mathrm{I}, \mathrm{J}+2)$

GO TO 140

ENDIF

IF(I.NE. $(\mathrm{N}-2))$ THEN

$\operatorname{ALF} 1=2 . /(\operatorname{APSI}(\mathrm{J}+1) *(\operatorname{SIG}(\mathrm{I}-1, \mathrm{~J}+1)+\operatorname{SIG}(\mathrm{I}+1, \mathrm{~J}+1)))$

$\operatorname{BLADO}(\mathrm{K})=-\mathrm{ALF} 1 * \mathrm{X}(\mathrm{I}, \mathrm{J}+2)$

GO TO 140

ENDIF

$\mathrm{ALF} 1=2 . /(\operatorname{APSI}(\mathrm{J}+1) *(\operatorname{SIG}(\mathrm{I}-1, \mathrm{~J}+1)+\operatorname{SIG}(\mathrm{I}+1, \mathrm{~J}+1)))$

$\operatorname{ALF} 2=(\operatorname{APSI}(J-1) * \operatorname{SIG}(I+1, J-1)+\operatorname{APSI}(J+1) * \operatorname{SIG}(I+1, \mathrm{~J}+1)) / 2$.

$A L F 2=(\mathrm{HP} / \mathrm{H}) *(\mathrm{HP} / \mathrm{H}) * \mathrm{ALF} 2$

$\operatorname{BLADO}(\mathrm{K})=-\operatorname{ALF} 1 * \mathrm{X}(\mathrm{I}, \mathrm{J}+2)-\mathrm{ALF} 2 * \mathrm{X}(\mathrm{I}+2, \mathrm{~J})$ 
$\mathrm{C}$

$\mathrm{C}$

SUB-ROTINA SUBSOL

C
C
C
C
C

Chama a biblioteca NAG e resolve o sistema de equações lineares $M X=\mathbb{B}$ para o vetor $\mathrm{X}$. A solução sai em $\mathbb{R H S}$

SUBROUTINE SUBSOL(A,IRN,ICN,N,M,LIRN,LICN,RHS,NEQ,NZ)

PARAMETER ( NEQ1 $=1501)$

REAL A(LICN),RHS(NEQ1),W(NEQ1),U,RESID

INTEGER IRN(LIRN),ICN(LICN),IKEEP(5*NEQ1),IDISP(20),

* IW(8*NEQ1),IFAIL

INTEGER N,M,LIRN,LICN,NZ,NEQ

C

LOGICAL LBLOCK,GROW,ABORT(4)

$\mathrm{U}=0.1$

LBLOCK $=$.TRUE.

GROW $=$.TRUE.

ABORT $(1)=$.TRUE.

$A B O R T(2)=. T R U E$.

$A B O R T(3)=$. .FALSE

ABORT(4) $=$.TRUE.

C

...Decomposição LU de uma permutação de $\mathbb{M}$, PMQ-LU.

CALL F01BRF(NEQ,NZ,A,LICN,IRN,LIRN,ICN,U,IKEEP,IW,W,LBLOCK,GROW, * ABORT,IDISP,IFAIL)

TYPE *,' IDISP(2)=',IDISP(2)

TYPE *,' IDISP(3)=',IDISP(3)

TYPE *,' IDISP(4)=',IDISP(4)

TYPE *,' IDISP(6) $=$,'IDISP(6)

TYPE *,' IDISP(7) $=$,'IDISP(7)

IF(GROW) TYPE *,' W(1)=',W(1)

C IF(LBLOCK) TYPE *,' IDISP(8:10)=',(IDISP(I),I=8,10)

CALL F04AXF(NEQ,A,LICN,ICN,IKEEP,RHS, W,MTYPE,IDISP,RESID) RETURN

END

\section{Programa Taylor}

$C$
$C$
$C$
$C$
$C$
$C$
$C$

\section{PROGRAMA TAYLOR. FOR}

*************************

Este programa integra as equações de carga espacial expandidas em séries de Taylor truncadas em segunda ordem num dominio simplesmente conexo. Ver desenvolvimento formal no Cap.III .

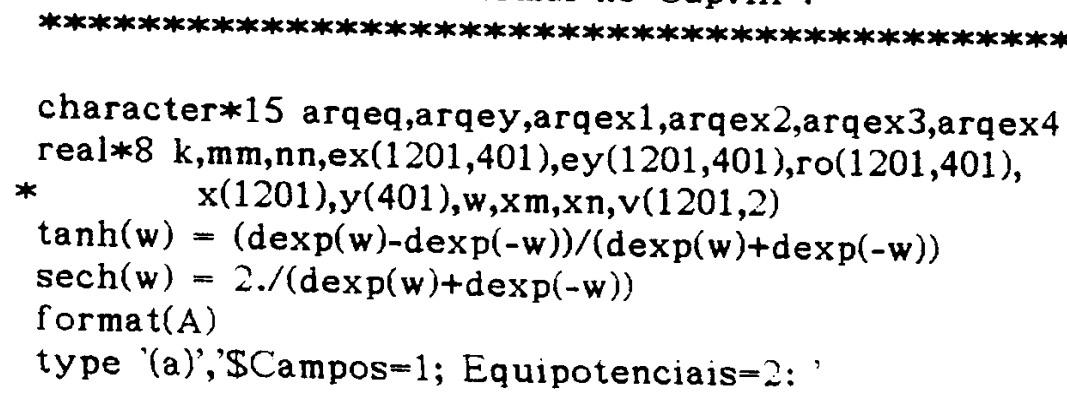


accept $*, 1$

if(1.eq.1) then

type '(A)','SArquivo para gravar ey( $\mathrm{x}=0)$ :'

accept 10000 ,arqey

open(unit=15, status='new',file=arqey)

type '(A)', 'SArquivo para gravar ex $(y=1 / 4)$ : '

accept 10000 ,arqex1

open(unit=11,status='new',file=arqex1)

type '(A)','SArquivo para gravar ex(y=1/2): '

accept 10000 ,arqex2

open(unit $=12$,status='new', file=arqex2)

type '(A)','SArquivo para gravar ex $(y=3 / 4)$ : '

accept 10000 ,arqex 3

open(unit=13,status='new',file=arqex3)

type '(A)', 'SArquivo para gravar ex $(y=1)$ :'

accept 10000 ,arqex 4

open(unit $=14$,status='new',file=arqex 4 )

$\mathrm{ml}=0$

else

type '(A)', 'SArquivo para gravar $y(x)$ da equipotencial: ' accept 10000 ,arqeq

open(unit=10,status='new',file-arqeq)

type '(a)', \$v0 (da sup. equipotencial) - NEGATIVO!: '

accept $*$, v0

type '(a)','\$Quantos dely quer ir alem de $y=p i$ ?'

accept $*, \mathrm{ml}$

endif

type '(a)','\$rho0: '

accept $*$,rhoo

type '(a)','\$mm, nn:'

accept $*, \mathrm{~mm}, \mathrm{nn}$

type '(a)', $\$$ m,n (m multiplo de 4 se a opcao for Campo):'

accept $*, \mathrm{~m}, \mathrm{n}$

type '(a)','\$delx/dely:'

accept $*$,delxdely

type '(a)', $\$ \mathrm{XX}$ :'

accept $*, \mathrm{X} 0$

dely $=1 . / \mathrm{m}$

del $x=$ delxdely $*$ dely

$\mathrm{xi}=-\mathrm{n} * \mathrm{del} \mathrm{x}$

$x s=n *$ del $x$

rhoi $=\operatorname{rho} 0 *(2 . *(1 .-\tanh (n n * x i))+.5)$

rhos $=\operatorname{rho} 0 *(2 . *(1 .-\tanh (n n * x s))+.5)$

$\mathrm{A} 0=1$.

$\mathrm{A} 1=2 . * \mathrm{rhoi}-1$.

$\mathrm{A} 2=4$,*rhoi $* \mathrm{rhoi} / 3$.

$\mathrm{A} 3=-3 . * \mathrm{rhoi} / 2$.

$\mathrm{H} 0=(3 . * \mathrm{~A} 0 * \mathrm{~A} 2-\mathrm{A} 1 * \mathrm{~A} 1) /(9 . * \mathrm{~A} 0 * \mathrm{~A} 0)$

$\mathrm{G}=2 . *((\mathrm{~A} 1 /(3 . * \mathrm{~A} 0)) * * 3)-\mathrm{A} 1 * \mathrm{~A} 2 /(3 . * \mathrm{~A} 0 * \mathrm{~A} 0)+\mathrm{A} 3 / \mathrm{A} 0$

$\mathrm{U} 0=(-\mathrm{G}+\mathrm{SQRT}(\mathrm{G} * \mathrm{G}+4 . * \mathrm{H} 0 * \mathrm{H} 0 * \mathrm{H} 0)) / 2$.

$\mathrm{U} 13=\mathrm{U} 0 * *(1 . / 3$.

$\mathrm{E} 0 \mathrm{i}=\mathrm{U} 13-\mathrm{H} 0 / \mathrm{U} 13-\mathrm{A} 1 /(3 . * \mathrm{~A} 0)$

type $*,{ }^{3} \mathrm{EOi}={ }^{\prime}, \mathrm{EOi}$

$\mathrm{A} 0=1$.

$\mathrm{A} 1=2 . * \mathrm{rhos}-1$.

$\mathrm{A} 2=4 . * \mathrm{rhos} * \mathrm{rhos} / 3$.

$\mathrm{A} 3=-3 . * \mathrm{rhos} / 2$.

$\mathrm{H} 0=(3 . * \mathrm{~A} 0 * \mathrm{~A} 2-\mathrm{A} 1 * \mathrm{~A} 1) /(9 . * \mathrm{~A} 0 * \mathrm{~A} 0)$

$\mathrm{G}=2 . *((\mathrm{~A} 1 /(3 . * \mathrm{~A} 0)) * * 3)-\mathrm{A} 1 * \mathrm{~A} 2 /(3 . * \mathrm{~A} 0 * \mathrm{~A} 0)+\mathrm{A} 3 / \mathrm{A} 0$

$\mathrm{UO}=(-\mathrm{G}+\mathrm{SQRT}(\mathrm{G} * \mathrm{G}+4 . * \mathrm{H} 0 * \mathrm{H} 0 * \mathrm{H} 0)) / 2$.

$\mathrm{U} 13=\mathrm{U} 0 * *(1 . / 3$.)

$\mathrm{E} 0 \mathrm{~s}=\mathrm{U} 13-\mathrm{H} 0 / \mathrm{U} 13-\mathrm{A} 1 /(3 . * \mathrm{~A} 0)$

type *,'E0s=',EOs 
$\mathrm{k}=.5 *(\mathrm{E} 0 \mathrm{~s}-\mathrm{E} 0 \mathrm{i}) / \mathrm{E} 0 \mathrm{i}$

type $*,{ }^{\prime} k=', k$

$\mathrm{m}=\mathrm{m}+\mathrm{ml}$

$j=1$

do $1000 i=1,(2 * n+1)$

$\mathrm{ii}=\mathrm{i}-(\mathrm{n}+1)$

$\mathrm{x}(\mathrm{i})=\mathrm{ii} * \mathrm{del} \mathrm{x}$

$\operatorname{ex}(\mathrm{i}, 1)=0$

$\mathrm{xm}=\mathrm{mm} * \mathrm{x}(\mathrm{i})$

if (xm.le.87..and.xm.ge.-87.) then

$\operatorname{ey}(\mathrm{i}, 1)=\mathrm{E} 0 \mathrm{i} *(1 .+\mathrm{k} *(1 .+\tanh (\mathrm{mm} *(\mathrm{x}(\mathrm{i})-\mathrm{X} 0))))$

else if (xm.gt.87) then

$e y(\mathrm{i}, 1)=\mathrm{E} 0 \mathrm{i} *(1 .+2 . * \mathrm{k})$

else

$e y(i, 1)=E O i$

endif

$\mathrm{xn}=\mathrm{nn} * \mathrm{x}(\mathrm{i})$

if (xn.le.87..and.xn.ge.-87.) then

$\operatorname{ro}(\mathrm{i}, 1)=\operatorname{rho} 0 *(2 . *(1 .-\tanh (\operatorname{nn} * x(\mathrm{i})))+.5)$

C $\quad \operatorname{ro}(\mathrm{i}, 1)=\operatorname{rho} 0 *(2 . *(1 .-\tanh (n n * x(i)))+.25)$

else if (xn.gt.87) then

ro $(i, 1)=.5 *$ rhoo

C $\quad$ ro(i,1) $=.25 *$ rho0

else

$\mathrm{ro}(\mathrm{i}, 1)=4.5 * \mathrm{rho0}$

C $\quad$ ro $(\mathrm{i}, 1)=.25 * \mathrm{rho}$

endif

1000 continue

do $500 \mathrm{j}=1, \mathrm{~m}+1$

$\mathrm{jj}=\mathrm{j}-1$

$y(j)=j j *$ dely

500 continue

C eyexato $=(1 .+\mathrm{k}) * \operatorname{sqrt}(1 .+\mathrm{c} * \mathrm{y}(1) /(1 .+\mathrm{k}))$

if (l.eq.1) then

write $(15, *), y(1), e y(n+1,1)$

endif

C type $*, \operatorname{ex}(n / 2,1), \operatorname{ex}(n+1,1), \operatorname{ex}(3 * n / 2+1,1)$

a1 $=0$.

a $3=0$

do $1500 \mathrm{i}=2,2 * \mathrm{n}$

$\mathrm{xm}=\mathrm{mm} *(\mathrm{x}(\mathrm{i})-\mathrm{X} 0)$

$\mathrm{xn}=\mathrm{nn} * \mathrm{x}(\mathrm{i})$

if (xm.le.87...and.xm.ge.-87.) then

$\mathrm{b} 1=E 0 i * \mathrm{k} * \mathrm{~mm} * \operatorname{sech}(\mathrm{xm}) * \operatorname{sech}(\mathrm{xm})$

b3 $=-E 0 i * k * m m * m m * \operatorname{sech}(x m) * \operatorname{sech}(x m) * \tanh (x m)$

else

$\mathrm{b} 1=0$.

$\mathrm{b} 3=0$.

endif

if ( $x$ n.le.87..and.xn.ge.-87) then

$c 1=-2 . * r h o 0 * n n * \operatorname{sech}(x n) * \operatorname{sech}(x n)$

c3 $=2 . * \mathrm{rho} 0 * \mathrm{n} n * \mathrm{n} n * \operatorname{sech}(\mathrm{xn}) * \operatorname{sech}(\mathrm{xn}) * \tanh (\mathrm{xn})$

else

$\mathrm{c} 1=0$.

$c 3=0$.

endif

$c 2=-(r o(i, 1) * r o(i, 1)+c 1 * \operatorname{ex}(i, 1)) / e y(i, 1)$

b5 $=-b 3+c 2 / 2$.

$c 4=-2 . * c 1 * r o(i, 1) / e y(i, 1)-b 1 * c 2 / e y(i, 1)-a 1 * c 1 / e y(i, 1)-$

* $\quad 2 . * \operatorname{ex}(\mathrm{i}, 1) * \mathrm{c} 3 / \mathrm{ey}(\mathrm{i}, 1)$

$\mathrm{c5}=.5 *(\mathrm{a} 1 / \mathrm{ey}(\mathrm{i}, 1)-3 . * \mathrm{ro}(\mathrm{i}, 1) / \mathrm{ey}(\mathrm{i}, 1)) * \mathrm{c} 2-.5 * \mathrm{~b} 1 * \mathrm{c} 1 / \mathrm{ey}(\mathrm{i}, 1)-$

$* \quad .5 * \operatorname{ex}(\mathrm{i}, 1) * c 4 / \mathrm{ey}(\mathrm{i}, 1)$

dely 2 = dely*dely 


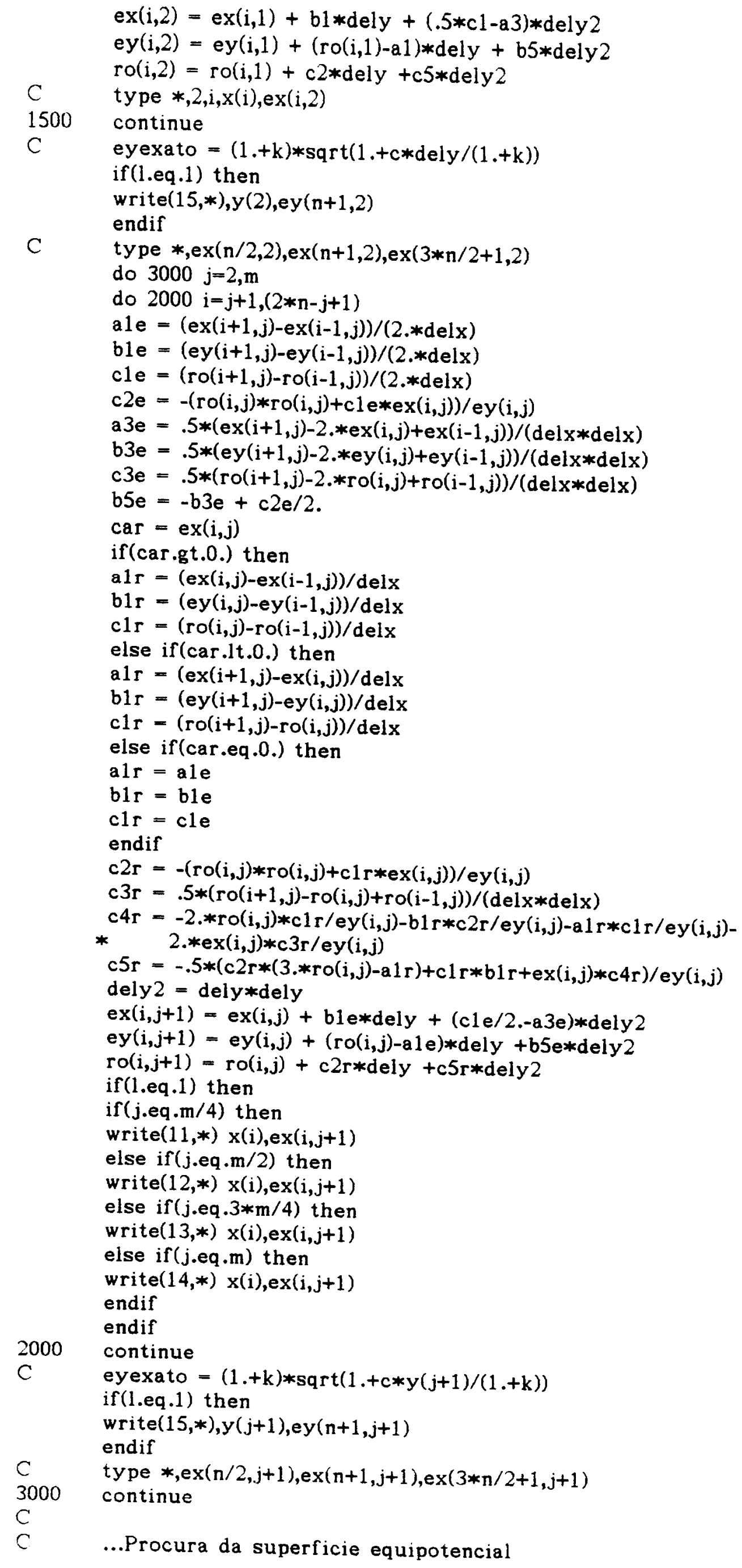


C

if (1.eq.2) then

$\mathrm{mf}=(2 * \mathrm{n}+1)-\mathrm{m}$

do $6000 \mathrm{i}=\mathrm{m}, \mathrm{mf}$

$v(i, 1)=1$.

$v(\mathrm{i}, 2)=1$.

do $5000 \mathrm{j}=1, \mathrm{~m}$

$v(i, 2)=v(i, 2)-(e y(i, j)+e y(i, j+1)) * \operatorname{dely} / 2$.

if $(v(i, 2) . l e . v 0)$ then

$y 0=((v 0-v(i, 1)) * y(j+1)+(v(i, 2)-v 0) * y(j)) /(v(i, 2)-v(i, 1))$

write $(10, *) \times(i), y 0$

goto 6000

endif

5000

$v(i, 1)=v(i, 2)$

6000

continue

continue

endif

stop

end 


\section{Referências Bibliográficas}

[1] Lampert, M. A. e Mark, P., Currente Injection in Solids. N. York, Academic Press, 1970.

[2] Many, A. e Rakavy, G., Phys. Rev. 126, (1962) 1980.

[3] Oliveira, L. N. e Leal Ferreira, G. F., J. Electrostatics 1 , (1975) 371.

[4] Budd, C. J. e Wheeler, A. A., Proc. R. Soc. Lond. A 417, (1 988) 389.

[5] Sigmond, R. S., J. Electrostatics 18, (1 986) 249.

[6] Godounov, S., Équations de la Physique Mathématique. Moscou, MIR, 1973.

[7] Leal Ferreira, G. F., J. Nonmetals 2, (1 974) 109.

[8] Felici, N. J., Direct Current, sept., (1963) 252.

[9] Felici, N. J., Direct Current, oct., (1 963) 278.

[10] Atten, P., C. R. Acad. Sci. Paris B 266, (1 968) 1188.

[11] Atten, P., R. G. E. Tome 83, N• 3, (1 974) 143.

[12] Sarma, M. P. e Janischewskyj, W., IEEE Trans. Power Apparatus and Systems, PAS-88, 5, (1 969) 718.

[13] Sarma, M. P. e Janischewskyj, W., IEEE Trans. Power Apparatus and Systems, PAS-88, 10, (1969) 1476.

[14] Smith, S. A., IMA J. Appl. Math. 39, (1 987) 189.

[15] Varley, E., Oxford Study Group, report (1985).

[16] Budd, C. J. e Wheeler, A. A., IMA J. Appl. Math. 40, (1988) 1.

[17] Deutsch, W., Ann. Phys. (Leipzig) 16, (1 933) 588.

[18] Popkov, V. I., Dok1. 58, (1 947) 1043.

[19] Popkov, V. I., Electrichestvo 1, (1949) 33.

[20] Popkov, V. I. e Ryabaya, S. I., Electrichestvo 11, (1 974) 45.

[21] Sigmond, R. S., J. Appl. Phys. Vol. 53, 2, (1 982) 892.

[22] Walsh, P. J., Gallo, C. F. e Lama, W., Photogr. Sci. Eng., 28, (1 984) 109.

[23] Janischewskyj, W., Sarma, M. P. e Gela, G. CIGRE paper 336-09, (1 982).

[24] Takuma, T., Ikeda, T. e Kowamoto, T., IEEE Trans. Power Appar. Syst., PAS - 100 (12) (1 981) 4802.

[25] Jacobs, D. A. H., PDEs. CERL (Letherhead, London) RD/L/N 66/72 (1 972).

[26] Budd, C. J., Friedman, A., McLeod, B. e Wheeler, A. A., SIAM J. Appl. Math. Vol. 50, 1, (1990) 181.

[27] Smith, G. D., Numerical Solution of Partial Differential Equations, Oxford University Press (1965).

[28] Dantas, I. F., Leal Ferreira, G. F. e Figueiredo, M. T. - Cálculo Aproximado da Linha de Corrente Extrema em um Eletródio Parcialmente Emissor. In: XIII Encontro Nac. de Fis. da Mat. Condensada, Caxambu, 8-12 maio (1 990). 